\title{
Tables of Pure Quintic Fields
}

\author{
Daniel C. Mayer \\ Naglergasse 53, Graz, Austria \\ Email: quantum.algebra@icloud.com
}

How to cite this paper: Mayer, D.C. (2019) Tables of Pure Quintic Fields. $A d$ vances in Pure Mathematics, 9, 347-403. https://doi.org/10.4236/apm.2019.94017

Received: December 11, 2018

Accepted: April 26, 2019

Published: April 29, 2019

Copyright (c) 2019 by author(s) and Scientific Research Publishing Inc. This work is licensed under the Creative Commons Attribution International License (CC BY 4.0).

http://creativecommons.org/licenses/by/4.0/

\begin{abstract}
By making use of our generalization of Barrucand and Cohn's theory of principal factorizations in pure cubic fields $\mathbb{Q}(\sqrt[3]{D})$ and their Galois closures $\mathbb{Q}\left(\zeta_{3}, \sqrt[3]{D}\right)$ with 3 possible types to pure quintic fields $L=\mathbb{Q}(\sqrt[5]{D})$ and their pure metacyclic normal fields $N=\mathbb{Q}\left(\zeta_{5}, \sqrt[5]{D}\right)$ with 13 possible types, we compile an extensive database with arithmetical invariants of the 900 pairwise non-isomorphic fields $N$ having normalized radicands in the range $2 \leq D<10^{3}$. Our classification is based on the Galois cohomology of the unit group $U_{N}$, viewed as a module over the automorphism group $\operatorname{Gal}(N / K)$ of $N$ over the cyclotomic field $K=\mathbb{Q}\left(\zeta_{5}\right)$, by employing theorems of Hasse and Iwasawa on the Herbrand quotient of the unit norm index $\left(U_{K}: N_{N / K}\left(U_{N}\right)\right)$ by the number $\#\left(\mathcal{P}_{N / K} / \mathcal{P}_{K}\right)$ of primitive ambiguous principal ideals, which can be interpreted as principal factors of the different $\mathfrak{D}_{N / K}$. The precise structure of the $\mathbb{F}_{5}$-vector space of differential principal factors is expressed in terms of norm kernels and central orthogonal idempotents. A connection with integral representation theory is established via class number relations by Parry and Walter involving the index of subfield units $\left(U_{N}: U_{0}\right)$. The statistical distribution of the 13 principal factorization types and their refined splitting into similarity classes with representative prototypes is discussed thoroughly.
\end{abstract}

\section{Keywords}

Pure Quintic Fields, Pure Metacyclic Fields, Units, Galois Cohomology, Differential Principal Factorization Types, Similarity Classes, Prototypes, Class Group Structure

\section{Introduction}

At the end of his 1975 article on class numbers of pure quintic fields, Parry 
suggested verbatim: In conclusion, the author would like to say that he believes a numerical study of pure quintic fields would be most interesting ([1] p. 484). Of course, it would have been rather difficult to realize Parry's desire in 1975. But now, 40 years later, we are in the position to use the powerful computer algebra systems PARI/GP [2] and MAGMA [3] [4] [5] for starting an attack against this hard problem. Prepared by [6] [7] [8] [9], this will actually be done in the present paper.

Even in 1991, when we generalized Barrucand and Cohn's theory [10] [11] of principal factorization types from pure cubic fields $\mathbb{Q}(\sqrt[3]{D})$ to pure quintic fields $L=\mathbb{Q}(\sqrt[5]{D})$ and their pure metacyclic normal closures $N=\mathbb{Q}\left(\zeta_{5}, \sqrt[5]{D}\right)$ [12], it was still impossible to verify our hypothesis about the distinction between absolute, intermediate and relative differential principal factors (DPF) ([6] (6.3)) and about the values of the unit norm index $\left(U_{K}: N_{N / K}\left(U_{N}\right)\right) \quad([6]$ (1.3)) by actual computations.

All these conjectures have been proven by our most recent numerical investigations. Our classification is based on the Hasse-Iwasawa theorem about the Herbrand quotient of the unit group $U_{N}$ of the Galois closure $N$ of $L$ as a module over the relative group $G=\operatorname{Gal}(N / K)$ with respect to the cyclotomic subfield $K=\mathbb{Q}\left(\zeta_{5}\right)$. It only involves the unit norm index $\left(U_{K}: N_{N / K}\left(U_{N}\right)\right)$ and our 13 types of differential principal factorizations ([6] Thm. 1.3), but not the index of subfield units $\left(U_{N}: U_{0}\right)([6] \S 5)$ in Parry's class number formula ([6] (5.1)).

We begin with a collection of explicit multiplicity formulas in $\S 2$ which are required for understanding the subsequent extensive presentation of our computational results in twenty tables of crucial invariants in $\S 3$. This information admits the classification of all 900 pure quintic fields $L=\mathbb{Q}(\sqrt[5]{D})$ with normalized radicands $2 \leq D<10^{3}$ into 13 DPF types and the refined classification into similarity classes with representative prototypes in $\S 4$.

We draw the attention to remaining open questions in $\S 3.3$, and we collect theoretical consequences of our experimental results in $\S 4.3$. The exposition is concluded with a retrospective final $\S 5$.

\section{Collection of Multiplicity Formulas}

For the convenience of the reader, we provide a summary of formulas for calculating invariants of pure quintic fields $L=\mathbb{Q}(\sqrt[5]{D})$ with normalized fifth power free radicands $D>1$ and their associated pure metacyclic normal fields $N=\mathbb{Q}(\zeta, \sqrt[5]{D})$ with a primitive fifth root of unity $\zeta=\zeta_{5}$.

Let $f$ be the class field theoretic conductor of the relatively quintic Kummer extension $N / K$ over the cyclotomic field $K=\mathbb{Q}(\zeta)$. It is also called the conductor of the pure quintic field $L$. The multiplicity $m=m(f)$ of the conductor $f$ indicates the number of non-isomorphic pure metacyclic fields $N$ sharing the common conductor $f$, or also, according to ([6] Prop. 2.1), the number of normalized fifth power free radicands $D>1$ whose fifth roots 
generate non-isomorphic pure quintic fields $L$ sharing the common conductor $f$. The cardinality of a set $S$ is denoted \# .

We adapt the general multiplicity formulas in ([13] Thm. 2, p. 104) to the quintic case $p=5$. If $L$ is a field of species 1a ([6] (2.6) and Exm. 2.2), i.e. $f^{4}=5^{6} \cdot q_{1}^{4} \cdots q_{t}^{4}$, then $m=4^{t}$ where $t:=\#\{q \in \mathbb{P}|q \neq 5, q| f\}$. The explicit values of $m$ in dependence on $t$ are given in Table 1 .

If $L$ is a field of species $1 \mathrm{~b}$ ([6] (2.6) and Exm. 2.2), i.e. $f^{4}=5^{2} \cdot q_{1}^{4} \cdots q_{t}^{4}$, then $m=4^{u} \cdot X_{v}$ where $u:=\#\{q \in \mathbb{P}|q \equiv \pm 1, \pm 7 \bmod (25), q| f\}, v:=t-u$ and $X_{j}:=\frac{1}{5}\left(4^{j}-(-1)^{j}\right)$, that is $\left(X_{j}\right)_{j \geq-1}=\left(\frac{1}{4}, 0,1,3,13,51,205, \cdots\right)$. The explicit values of $m$ in dependence on $u$ and $v$ are given in Table 2 .

If $L$ is a field of species 2 ([6] (2.6) and Exm. 2.2), i.e. $f^{4}=5^{0} \cdot q_{1}^{4} \cdots q_{t}^{4}$, then $m=4^{u} \cdot X_{v-1}$ where $u:=\#\{q \in \mathbb{P}|q \equiv \pm 1, \pm 7 \bmod (25), q| f\}, \quad v:=t-u \quad$ and $X_{j}:=\frac{1}{5}\left(4^{j}-(-1)^{j}\right)$, that is $\left(X_{j}\right)_{j \geq-1}=\left(\frac{1}{4}, 0,1,3,13,51,205, \cdots\right)$. The explicit values of $m$ in dependence on $u$ and $v$ are given in Table 3 .

\section{Classification by DPF Types in 20 Numerical Tables}

\subsection{DPF Types}

The following twenty Tables 6-25 establish a complete classification of all 900 pure metacyclic fields $N=\mathbb{Q}(\zeta, 5 D)$ with normalized radicands in the range $2 \leq D<10^{3}$. With the aid of PARI/GP [2] and MAGMA [5] we have determined the differential principal factorization type, $\mathrm{T}$, of each field $N$ by means of other invariants $U, A, I, R$ ([6] Thm. 6.1). After several weeks of CPU time, the date of completion was September 17, 2018.

The possible DPF types are listed in dependence on $U, A, I, R$ in Table 4, where the symbol $\times$ in the column $\eta$, resp. $\zeta$, indicates the existence of a unit $H \in U_{N}$, resp. $Z \in U_{N}$, such that $\eta=N_{N / K}(H)$, resp. $\zeta=N_{N / K}(Z)$. The 5-valuation of the unit norm index $\left(U_{K}: N_{N / K} U_{N}\right)$ is abbreviated by $U$ ([6] (1.3), (6.3)]. Here, $\eta=\frac{1}{2}(1+\sqrt{5})$ denotes the fundamental unit of $K^{+}=Q(\sqrt{5})$.

Table 1. Multiplicity of fields of species 1a.

\begin{tabular}{ccccccc}
\hline$t$ & 0 & 1 & 2 & 3 & 4 & 5 \\
\hline$m$ & 1 & 4 & 16 & 64 & 256 & 1024 \\
\hline
\end{tabular}

Table 2. Multiplicity of fields of species $1 \mathrm{~b}$.

\begin{tabular}{cccccccc}
\hline$u$ & $V$ & 0 & 1 & 2 & 3 & 4 & 5 \\
\hline 0 & $m$ & 0 & 1 & 3 & 13 & 51 & 205 \\
1 & & 0 & 4 & 12 & 52 & 204 & 820 \\
2 & & 0 & 16 & 48 & 208 & 816 & \\
3 & & 0 & 64 & 192 & 832 & & \\
4 & & 0 & 256 & 768 & & & \\
\hline
\end{tabular}


Table 3. Multiplicity of fields of species 2 .

\begin{tabular}{cccccccc}
\hline$u$ & $v$ & 0 & 1 & 2 & 3 & 4 & 5 \\
\hline 0 & $m$ & 0 & 0 & 1 & 3 & 13 & 51 \\
1 & 1 & 0 & 4 & 12 & 52 & 204 \\
2 & 4 & 0 & 16 & 48 & 208 & 816 \\
3 & & 16 & 0 & 64 & 192 & 832 & \\
4 & 64 & 0 & 256 & 768 & & \\
\hline
\end{tabular}

Table 4. Differential principal factorization types, T, of pure metacyclic fields $N$.

\begin{tabular}{lllllll}
\hline $\mathrm{T}$ & $U$ & $\eta$ & $\zeta$ & $A$ & $I$ & $R$ \\
\hline$\alpha_{1}$ & 2 & - & - & 1 & 0 & 2 \\
$\alpha_{2}$ & 2 & - & - & 1 & 1 & 1 \\
$\alpha_{3}$ & 2 & - & - & 1 & 2 & 0 \\
$\beta_{1}$ & 2 & - & - & 2 & 0 & 1 \\
$\beta_{2}$ & 2 & - & - & 2 & 1 & 0 \\
$\gamma$ & 2 & - & - & 3 & 0 & 0 \\
$\delta_{1}$ & 1 & $\times$ & - & 1 & 0 & 1 \\
$\delta_{2}$ & 1 & $\times$ & - & 1 & 1 & 0 \\
$\varepsilon$ & 1 & $\times$ & - & 2 & 0 & 0 \\
$\zeta_{1}$ & 1 & - & $\times$ & 1 & 0 & 1 \\
$\zeta_{2}$ & 1 & - & $\times$ & 1 & 1 & 0 \\
$\eta$ & 1 & - & $\times$ & 2 & 0 & 0 \\
$\vartheta$ & 0 & $\times$ & $\times$ & 1 & 0 & 0 \\
\hline
\end{tabular}

\subsection{Justification of the Computational Techniques}

The steps of the following classification algorithm are ordered by increasing requirements of CPU time. To avoid unnecessary time consumption, the algorithm stops at early stages already, as soon as the DPF type is determined unambiguously. The illustrating subfield lattice of $N$ is drawn in Figure 1.

\section{Algorithm 3.1 (Classification into 13 DPF types.)}

Input: a normalized fifth power free radicand $D \geq 2$.

Step 1: By purely rational methods, without any number field constructions, the prime factorization of the radicand $D$ (including the counters $t, u, v ; n, s_{2}, s_{4}$, $\S 4.2)$ is determined. If $D=q \in \mathbb{P}, q \equiv \pm 2 \bmod (5), q \neq \pm 7 \bmod (25)$, then $N$ is a Polya field of type $\varepsilon$; stop. If $D=q \in \mathbb{P}, q=5$ or $q \equiv \pm 7 \bmod (25)$, then $N$ is a Polya field of type $\vartheta$; stop.

Step 2: The field $L$ of degree 5 is constructed. The primes $q_{1}, \cdots, q_{T}$ dividing the conductor $f$ of $N / K$ are determined, and their overlying prime ideals $\mathfrak{q}_{1}, \cdots, \mathfrak{q}_{T}$ in $L$ are computed. By means of at most $5^{T}$ principal ideal tests of 

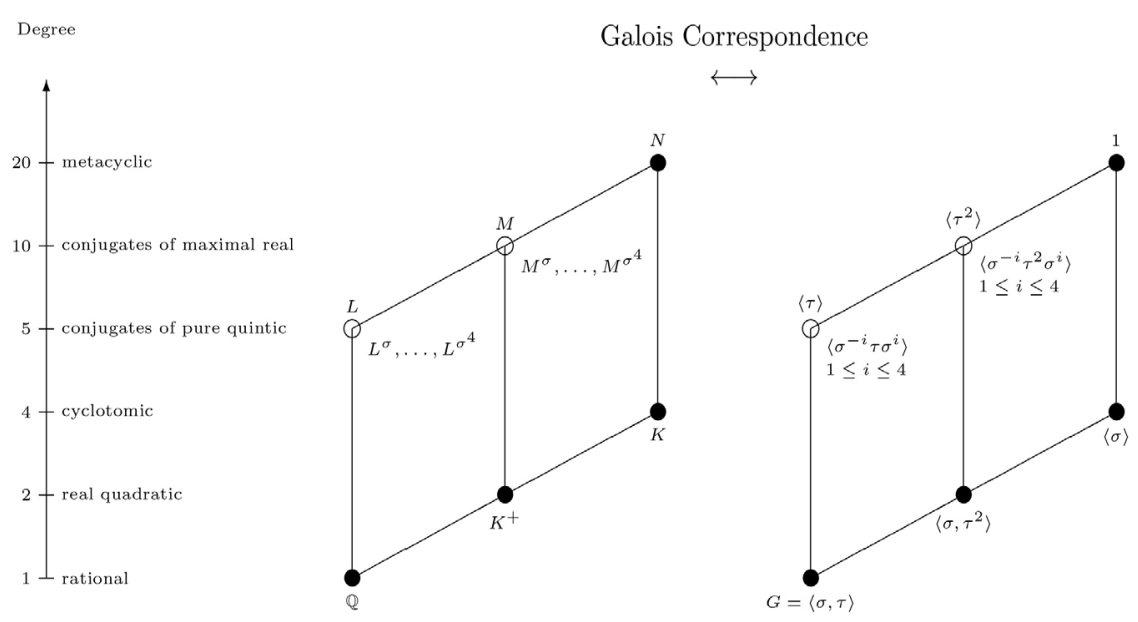

Figure 1. Lattices of subfields of $N$ and of subgroups of $G=\operatorname{Gal}(N / \mathbb{Q})$.

the elements of $\mathcal{I}_{L / \mathbb{Q}} / \mathcal{I}_{\mathbb{Q}}=\bigoplus_{i=1}^{T} \mathbb{F}_{5} \mathfrak{q}_{i}$, the number $5^{A}:=\#\left\{\left(v_{1}, \cdots, v_{T}\right) \in \mathbb{F}_{5}^{T} \mid \prod_{i=1}^{T} \mathfrak{q}_{i}^{v_{i}} \in \mathcal{P}_{L}\right\}$, that is the cardinality of $\mathcal{P}_{L / \mathbb{Q}} / \mathcal{P}_{\mathbb{Q}}$, is determined. If $A=T$, then $N$ is a Polya field. If $A=3$, then $N$ is of type $\gamma$; stop. If $A=2, s_{2}=s_{4}=0, v \geq 1$, then $N$ is of type $\varepsilon$; stop. If $A=1$, $s_{2}=s_{4}=0$, then $N$ is of type $\vartheta$; stop.

Step 3: If $s_{2} \geq 1$ or $s_{4} \geq 1$, then the field $M$ of degree 10 is constructed. For the 2 -split primes $\ell_{1}, \cdots, \ell_{s_{2}+s_{4}} \equiv \pm 1 \bmod (5)$ among the primes $q_{1}, \cdots, q_{T}$ dividing the conductor $f$ of $N / K$, the overlying prime ideals

$\mathcal{L}_{1}, \mathcal{L}_{1}^{\tau}, \cdots, \mathcal{L}_{s_{2}+s_{4}}, \mathcal{L}_{s_{2}+s_{4}}^{\tau}$ in $M$ are computed. By means of at most $5^{s_{2}+s_{4}}$ principal ideal tests of the elements of

$\left(\mathcal{I}_{M / K^{+}} / \mathcal{I}_{K^{+}}\right) \cap \operatorname{ker}\left(N_{M / L}\right)=\bigoplus_{i=1}^{s_{2}+s_{4}} \mathbb{F}_{5} \mathcal{K}_{\left(\ell_{i}\right)}$, where $\mathcal{K}_{\left(\ell_{i}\right)}=\mathcal{L}_{i}^{1+4 \tau}$ for $1 \leq i \leq s_{2}+s_{4}$, the number $5^{I}:=\#\left\{\left(v_{1}, \cdots, v_{s_{2}+s_{4}}\right) \in \mathbb{F}_{5}^{s_{2}+s_{4}} \mid \prod_{i=1}^{s_{2}+s_{4}} \mathcal{K}_{\left(\ell_{i}\right)}^{v_{i}} \in \mathcal{P}_{M}\right\}$, that is the cardinality of $\left(\mathcal{P}_{M / K^{+}} / \mathcal{P}_{K^{+}}\right) \cap \operatorname{ker}\left(N_{M / L}\right)$, is determined. If $I=2$, then $N$ is of type $\alpha_{3}$; stop. If $I=1, A=2$, then $N$ is of type $\beta_{2}$; stop.

Step 4: If $s_{4} \geq 1$, then the field $N$ of degree 20 is constructed. For all 4 -split primes $\ell_{s_{2}+1}, \cdots, \ell_{s_{2}+s_{4}} \equiv+1 \bmod (5)$ among the primes $q_{1}, \cdots, q_{T}$ dividing the conductor $f$ of $N / K$, the overlying prime ideals $\mathfrak{L}_{s_{2}+1}, \mathfrak{L}_{s_{2}+1}^{\tau^{2}}, \mathfrak{L}_{s_{2}+1}^{\tau}, \mathfrak{L}_{s_{2}+1}^{\tau^{3}}, \cdots, \mathfrak{L}_{s_{2}+s_{4}}, \mathfrak{L}_{s_{2}+s_{4}}^{\tau^{2}}, \mathfrak{L}_{s_{2}+s_{4}}^{\tau}, \mathfrak{L}_{s_{2}+s_{4}}^{\tau^{3}}$ in $N$ are computed. By means of at most $5^{2 s_{4}}$ principal ideal tests of the elements of $\left(\mathcal{I}_{N / K} / \mathcal{I}_{K}\right) \cap \operatorname{ker}\left(N_{N / M}\right)=\bigoplus_{i=s_{2}+1}^{s_{2}+s_{4}}\left(\mathbb{F}_{5} \mathfrak{K}_{1,\left(\ell_{i}\right)} \oplus \mathbb{F}_{5} \mathfrak{K}_{2,\left(\ell_{i}\right)}\right)$, where $\mathfrak{K}_{1,\left(\ell_{i}\right)}=\mathfrak{L}_{i}^{1+4 \tau^{2}+2 \tau+3 \tau^{3}}$ and $\mathfrak{K}_{2,\left(\ell_{i}\right)}=\mathfrak{L}_{i}^{\mathrm{l}+4 \tau^{2}+3 \tau+2 \tau^{3}}$ for $s_{2}+1 \leq i \leq s_{2}+s_{4}$, the number

$5^{R}:=\#\left\{\left(v_{1, s_{2}+1}, v_{2, s_{2}+1}, \cdots, v_{1, s_{2}+s_{4}}, v_{2, s_{2}+s_{4}}\right) \in \mathbb{F}_{5}^{2 s_{4}} \mid \prod_{i=s_{2}+1}^{s_{2}+s_{4}}\left(\mathfrak{K}_{1,\left(\ell_{i}\right)}^{v_{1, i}} \mathfrak{K}_{2,\left(\ell_{i}\right)}^{v_{2, i}}\right) \in \mathcal{P}_{N}\right\}$, that is the cardinality of $\left(\mathcal{P}_{N / K} / \mathcal{P}_{K}\right) \cap \operatorname{ker}\left(N_{N / M}\right)$, is determined. If $R=2$, then $N$ is of type $\alpha_{1}$; stop. If $R=1, I=1$, then $N$ is of type $\alpha_{2}$; stop. If $R=1, A=2$, then $N$ is of type $\beta_{1}$; stop.

Step 5: If the type of the field $N$ is not yet determined uniquely, then $U=1$ and there remain the following possibilities. If $v \geq 1$, then $N$ is of type $\delta_{1}$, if $R=1$, of type $\delta_{2}$, if $I=1$, and of type $\varepsilon$, if $R=I=0$. If $v=0$, then a 
fundamental system $\left(E_{j}\right)_{1 \leq j \leq 9}$ of units is constructed for the unit group $U_{N}$ of the field $N$ of degree 20, and all relative norms of these units with respect to the cyclotomic subfield $K$ are computed. If $N_{N / K}\left(E_{j}\right)=\zeta_{5}^{k}$ for some $1 \leq j \leq 9$, $1 \leq k \leq 4$, then $N$ is of type $\zeta_{1}$, if $R=1$, of type $\zeta_{2}$, if $I=1$, and of type $\eta$, if $R=I=0$. Otherwise the conclusions are the same as for $v \geq 1$.

Output: the DPF type of the field $N=\mathbb{Q}\left(\zeta_{5}, \sqrt[5]{D}\right)$ and the decision about its Polya property.

Proof. The claims of Step 1 concerning the types $\varepsilon, \vartheta$ are proved in items (1) and (2) of ([6] Thm. 10.1).

For Step 2, the formulas (4.1) and (4.2) in ([6] Thm. 4.1) give an $\mathbb{F}_{5}$-basis of the space of absolute differential factors, and the formulas (4.3) and (4.4) in ([6] Cor. 4.1) determine bounds for the $\mathbb{F}_{5}$-dimension $A$ of the space of absolute DPF in the field $L$ of degree 5. The Polya property was characterized in ([6] Thm. 10.5)], the claim concerning type $\gamma$ follows from ([6] Thm. 6.1), and the claims about the types $\varepsilon, \vartheta$ from ([6] Thm. 8.1 and Thm. 6.1).

For Step 3, the formulas (4.5) and (4.6) in ([6] Thm. 4.3) give an $\mathbb{F}_{5}$-basis of the space of intermediate differential factors, and the formulas (4.7) and (4.8) in ([6] Cor. 4.2) determine bounds for the $\mathbb{F}_{5}$-dimension $I$ of the space of intermediate DPF in the field $M$ of degree 10 . The claims concerning the types $\alpha_{3}, \beta_{2}$ are consequences of ([6] Thm. 6.1),

For Step 4, the formulas (4.9) and (4.10) in ([6] Thm. 4.4) give an $\mathbb{F}_{5}$-basis of the space of relative differential factors, and the formulas (4.11) and (4.12) in ([6] Cor. 4.3) determine bounds for the $\mathbb{F}_{5}$-dimension $R$ of the space of relative DPF in the field $N$ of degree 20. The claims concerning the types $\alpha_{1}, \alpha_{2}, \beta_{1}$ are consequences of ([6] Thm. 6.1).

Concerning Step 5, the signature of $N$ is $\left(r_{1}, r_{2}\right)=(0,10)$, whence the torsion free Dirichlet unit rank of $N$ is given by $r=r_{1}+r_{2}-1=9$. The claims about all types are consequences of ([6] Thm. 6.1), including information on the constitution of the norm group $N_{N / K}\left(U_{N}\right)$.

Remark 3.1 Whereas the execution of Step 1 and 2 in Algorithm 3.1, implemented as a Magma program [5], is a matter of a few seconds on a machine with two Intel XEON 8-core processors and clock frequency $2 \mathrm{GHz}$, the CPU time for Step 3 lies in the range of several minutes. The time requirement for Step 4 and 5 can reach hours or even days in spite of code optimizations for the calculation of units, in particular the use of the Magma procedures IndependentUnits() and SetOrderUnitsAreFundamental() prior to the call of UnitGroup().

\subsection{Open Problems}

We conjecture that considerable amounts of CPU time can be saved in our Algorithm 3.1 by computing the logarithmic 5-class numbers $V_{F}:=v_{5}\left(h_{F}\right)$ of the fields $F \in\{L, M, N\}$, which admit the determination of the logarithmic indices $E$, resp. $E^{+}$, of subfield units in the Parry [1], resp. Kobayashi [14] [15], 
class number relation, according to the formulas

$$
E=5+V_{N}-4 \cdot V_{L}, \quad E^{+}=2+V_{M}-2 \cdot V_{L} .
$$

However, first there would be required rigorous proofs of the heuristic connections between $E, E^{+}$and the DPF types in Table 5, where $\left(E, E^{+}\right)=(1,0)$ implies type $\alpha_{2},\left(E, E^{+}\right)=(2,0)$ implies type $\alpha_{3}$, $\left(E, E^{+}\right)=(4,2)$ implies type $\delta_{1}$, but $\left(E, E^{+}\right)=(2,1)$ admits types $\alpha_{1}, \delta_{1}$, $\left(E, E^{+}\right)=(3,1)$ admits types $\alpha_{2}, \beta_{1}, \delta_{2},\left(E, E^{+}\right)=(4,1)$ admits types $\beta_{2}, \zeta_{2}$, $\left(E, E^{+}\right)=(5,2)$ admits types $\beta_{1}, \varepsilon, \zeta_{1}, \vartheta$, and $\left(E, E^{+}\right)=(6,2)$ admits types $\gamma, \eta \cdot\left(E, E^{+}\right)=(0,0)$ seems to be impossible.

\subsection{Conventions and Notation in the Tables}

The normalized radicand $D=q_{1}^{e_{1}} \cdots q_{s}^{e_{s}}$ of a pure metacyclic field $N$ of degree 20 is minimal among the powers $D^{n}, 1 \leq n \leq 4$, with corresponding exponents $e_{j}$ reduced modulo 5 . The normalization of the radicands $D$ provides a warranty that all fields are pairwise non-isomorphic ([6] Prop. 2.1).

Prime factors are given for composite radicands $D$ only. Dedekind's species, $\mathrm{S}$, of radicands is refined by distinguishing $5 \mid D$ (species 1 a) and $\operatorname{gcd}(5, D)=1$ (species $1 b$ ) among radicands $D \not \equiv \pm 1, \pm 7 \bmod (25)$ (species 1 ). By the species and factorization of $D$, the shape of the conductor $f$ is determined. We give the fourth power $f^{4}$ to avoid fractional exponents. Additionally, the multiplicity $m$ indicates the number of non-isomorphic fields sharing a common conductor $f$ ( $\S 2$ ). The symbol $V_{F}$ briefly denotes the 5-valuation of the order $h_{F}=\# \mathrm{Cl}(F)$ of the class group $\mathrm{Cl}(F)$ of a number field $F$. By $E$ we denote the exponent of the power in the index of subfield units $\left(U_{N}: U_{0}\right)=5^{E}$.

Table 5. Logarithmic indices $E, E^{+}$of subfield units for DPF types, T.

\begin{tabular}{|c|c|c|c|c|c|}
\hline $\mathrm{T}$ & $E$ & $E^{+}$ & or & $E$ & $E^{+}$ \\
\hline$\alpha_{1}$ & 2 & 1 & & & \\
\hline$\alpha_{2}$ & 1 & 0 & & 3 & 1 \\
\hline$\alpha_{3}$ & 2 & 0 & & & \\
\hline$\beta_{1}$ & 3 & 1 & & 5 & 2 \\
\hline$\beta_{2}$ & 4 & 1 & & & \\
\hline$\gamma$ & 6 & 2 & & & \\
\hline$\delta_{1}$ & 2 & 1 & & 4 & 2 \\
\hline$\delta_{2}$ & 3 & 1 & & & \\
\hline$\varepsilon$ & 5 & 2 & & & \\
\hline$\zeta_{1}$ & 5 & 2 & & & \\
\hline$\zeta_{2}$ & 4 & 1 & & & \\
\hline$\eta$ & 6 & 2 & & & \\
\hline$\vartheta$ & 5 & 2 & & & \\
\hline
\end{tabular}


An asterisk denotes the smallest radicand with given Dedekind kind, DPF type and 5-class groups $\mathrm{Cl}_{5}(F), F \in\{L, M, N\}$. The latter are usually elementary abelian, except for the cases indicated by an additional asterisk (see $\S 4.4$ ).

Principal factors, $\mathrm{P}$, are listed when their constitution is not a consequence of the other information. According to ([6] Thm. 7.2., item (1)) it suffices to give the rational integer norm of absolute principal factors. For intermediate principal factors, we use the symbols $\mathcal{K}:=\mathcal{L}^{1-\tau}=\alpha \mathcal{O}_{M}$ with $\alpha \in M$ or $\mathcal{L}=\lambda \mathcal{O}_{M}$ with a prime element $\lambda \in M$ (which implies $\mathcal{L}^{\tau}=\lambda^{\tau} \mathcal{O}_{M}$ and thus also $\left.\mathcal{K}=\lambda^{1-\tau} \mathcal{O}_{M}\right)$. Here, $\left(\mathcal{L}^{1+\tau}\right)^{5}=\ell \mathcal{O}_{M}$ when a prime $\ell \equiv \pm 1 \bmod (5)$ divides the radicand $D$. For relative principal factors, we use the symbols $\mathfrak{K}_{1}:=\mathfrak{L}^{\mathfrak{l}+4 \tau^{2}+2 \tau+3 \tau^{3}}=A_{1} \mathcal{O}_{N} \quad$ and $\quad \mathfrak{K}_{2}:=\mathfrak{L}^{\mathfrak{l}+4 \tau^{2}+3 \tau+2 \tau^{3}}=A_{2} \mathcal{O}_{N} \quad$ with $\quad A_{1}, A_{2} \in N$. Here, $\left(\mathfrak{L}^{1+\tau+\tau^{2}+\tau^{3}}\right)^{5}=\ell \mathcal{O}_{N}$ when a prime number $\ell \equiv+1 \bmod (5)$ divides the radicand $D$. (Kernel ideals in [6] $\S 7$ )

The quartet $(1,2,4,5)$ indicates conditions which either enforce a reduction of possible DPF types or enable certain DPF types. The lack of a prime divisor $\ell \equiv \pm 1 \bmod (5)$ together with the existence of a prime divisor $q \neq \equiv \pm 7 \bmod (25)$ and $q \neq 5$ of $D$ is indicated by a symbol $\times$ for the component 1 . In these cases, only the two DPF types $\gamma$ and $\varepsilon$ can occur ([6] Thm. 8.1).

A symbol $\times$ for the component 2 emphasizes a prime divisor $\ell \equiv-1 \bmod (5)$ of $D$ and the possibility of intermediate principal factors in $M$, like $\mathcal{L}$ and $\mathcal{K}$. A symbol $\times$ for the component 4 emphasizes a prime divisor $\ell \equiv+1 \bmod (5)$ of $D$ and the possibility of relative principal factors in $N$, like $\mathfrak{K}_{1}$ and $\mathfrak{K}_{2}$. The $\times$ symbol is replaced by $\otimes$ if the facility is used completely, and by $(x)$ if the facility is only used partially.

If $D$ has only prime divisors $q \equiv \pm 1, \pm 7 \bmod (25)$ or $q=5$, a symbol $\times$ is placed in component 5. In these cases, $\zeta$ can occur as a norm $N_{N / K}(Z)$ of some unit in $Z \in U_{N}$. If it actually does, the $\times$ is replaced by $\otimes([6] \S 8)$.

\section{Statistical Evaluation and Refinements}

\subsection{Statistics of DPF Types}

The complete statistical evaluation of the following twenty Tables 6-25 is given in Table 26. The first ten columns show the absolute frequencies of pure metacyclic fields $N=\mathbb{Q}(\zeta, \sqrt[5]{D})$ with various DPF types for the ranges $2 \leq D<n \cdot 100$ with $1 \leq n \leq 10$. The eleventh column lists the relative percentages of the five most frequent DPF types for the complete range $2 \leq D<10^{3}$ of normalized radicands.

Among our 13 differential principal factorization types, type $\gamma$ with 3-dimensional absolute principal factorization, $A=3$, is clearly dominating with more than one third (36\%) of all occurrences in the complete range $2 \leq D<10^{3}$, followed by type $\varepsilon$ with 2 -dimensional absolute principal factorization, $A=2$, which covers nearly one quarter $(23 \%)$ of all cases. The third place (nearly $18 \%$ ) is occupied by type $\beta_{2}$ with mixed absolute and intermediate principal factorization, $A=2, I=1$. 
Table 6. 40 pure metacyclic fields with normalized radicands $2 \leq D \leq 52$.

\begin{tabular}{|c|c|c|c|c|c|c|c|c|c|c|c|c|}
\hline No. & $D$ & Factors & S & $f^{*}$ & $m$ & $V_{L}$ & $V_{M}$ & $V_{N}$ & $E$ & $(1,2,4,5)$ & $\mathrm{T}$ & $\mathrm{P}$ \\
\hline 1 & $\star 2$ & & $1 \mathrm{~b}$ & $5^{2} 2^{4}$ & 1 & 0 & 0 & 0 & 5 & $(\times,-,-,-)$ & $\varepsilon$ & \\
\hline 2 & 3 & & $1 \mathrm{~b}$ & $5^{2} 3^{4}$ & 1 & 0 & 0 & 0 & 5 & $(\times,-,-,-)$ & $\varepsilon$ & \\
\hline 3 & *5 & & $1 \mathrm{a}$ & $5^{6}$ & 1 & 0 & 0 & 0 & 5 & $(-,-,-, \otimes)$ & $\vartheta$ & \\
\hline 4 & ${ }^{*} 6$ & $2 \times 3$ & $1 \mathrm{~b}$ & $5^{2} 2^{4} 3^{4}$ & 3 & 0 & 0 & 1 & 6 & $(\times,-,-,-)$, & $\gamma$ & \\
\hline 5 & ${ }^{\star} 7$ & & 2 & $7^{4}$ & 1 & 0 & 0 & 0 & 5 & $(-,-,-, \otimes)$ & $\vartheta$ & \\
\hline 6 & $* 10$ & $2 \times 5$ & $1 \mathrm{a}$ & $5^{6} 2^{4}$ & 4 & 0 & 0 & 0 & 5 & $(\times,-,-,-)$ & $\varepsilon$ & \\
\hline 7 & ${ }^{*} 11$ & & $1 \mathrm{~b}$ & $5^{2} 11^{4}$ & 1 & 1 & 1 & 2 & 3 & $(-,-, \otimes,-)$ & $\alpha_{2}$ & $\mathcal{L}, \mathfrak{K}_{1}$ \\
\hline 8 & 12 & $2^{2} \times 3$ & $1 \mathrm{~b}$ & $5^{2} 2^{4} 3^{4}$ & 3 & 0 & 0 & 1 & 6 & $(\times,-,-,-)$ & $\gamma$ & \\
\hline 9 & 13 & & $1 \mathrm{~b}$ & $5^{2} 13^{4}$ & 1 & 0 & 0 & 0 & 5 & $(\times,-,-,-)$ & $\varepsilon$ & \\
\hline 10 & ${ }^{*} 14$ & $2 \times 7$ & $1 b$ & $5^{2} 2^{4} 7^{4}$ & 4 & 0 & 0 & 1 & 6 & $(\times,-,-,--)$ & $\gamma$ & \\
\hline 11 & 15 & $3 \times 5$ & la & $5^{6} 3^{4}$ & 4 & 0 & 0 & 0 & 5 & $(\times,-,-,-)$ & $\varepsilon$ & \\
\hline 12 & 17 & & $1 \mathrm{~b}$ & $5^{2} 17^{4}$ & 1 & 0 & 0 & 0 & 5 & $(\times,-,-,-)$ & $\varepsilon$ & \\
\hline 13 & ${ }^{\star} 18$ & $2 \times 3^{2}$ & 2 & $2^{4} 3^{4}$ & 1 & 0 & 0 & 0 & 5 & $(\times,-,-,-)$ & $\varepsilon$ & \\
\hline 14 & ${ }^{*} 19$ & & $1 \mathrm{~b}$ & $5^{2} 19^{4}$ & 1 & 1 & 1 & 2 & 3 & $(-, \otimes,-,-)$ & $\delta_{2}$ & $\mathcal{L}$ \\
\hline 15 & 20 & $2^{2} \times 5$ & $1 \mathrm{a}$ & $5^{6} 2^{4}$ & 4 & 0 & 0 & 0 & 5 & $(\times,-,-,-)$, & $\varepsilon$ & \\
\hline 16 & 21 & $3 \times 7$ & $1 \mathrm{~b}$ & $5^{2} 3^{4} 7^{4}$ & 4 & 0 & 0 & 1 & 6 & $(\times,-,-,-)$ & $\gamma$ & \\
\hline 17 & ${ }^{*} 22$ & $2 \times 11$ & $1 b$ & $5^{2} 2^{4} 11^{4}$ & 3 & 1 & 1 & 3 & 4 & $(-,-,(\times),-)$ & $\beta_{2}$ & $2 \times 5, \mathcal{K}$ \\
\hline 18 & 23 & & $1 \mathrm{~b}$ & $5^{2} 23^{4}$ & 1 & 0 & 0 & 0 & 5 & $(\times,-,-,-)$ & $\varepsilon$ & \\
\hline 19 & 26 & $2 \times 13$ & 2 & $2^{4} 13^{4}$ & 1 & 0 & 0 & 0 & 5 & $(\times,-,-,-)$ & $\varepsilon$ & \\
\hline 20 & 28 & $2^{2} \times 7$ & $1 \mathrm{~b}$ & $5^{2} 2^{4} 7^{4}$ & 4 & 0 & 0 & 1 & 6 & $(\times,-,-,-)$ & $\gamma$ & \\
\hline 21 & 29 & & $1 \mathrm{~b}$ & $5^{2} 29^{4}$ & 1 & 1 & 1 & 2 & 3 & $(-, \otimes,-,-)$ & $\delta_{2}$ & $\mathcal{L}$ \\
\hline 22 & $\star 30$ & $2 \times 3 \times 5$ & $1 \mathrm{a}$ & $5^{6} 2^{4} 3^{4}$ & 16 & 0 & 0 & 1 & 6 & $(\times,-,-,-)$ & $\gamma$ & \\
\hline 23 & $* 31$ & & $1 \mathrm{~b}$ & $5^{2} 31^{4}$ & 1 & 2 & 3 & 5 & 2 & $(-,-, \otimes,-)$ & $\alpha_{1}$ & $\mathfrak{K}_{1}, \mathfrak{K}_{2}$ \\
\hline 24 & $\star 33$ & $3 \times 11$ & $1 \mathrm{~b}$ & $5^{2} 3^{4} 11^{4}$ & 3 & 2 & 2 & 4 & 1 & $(-,-, \otimes,-)$ & $\alpha_{2}$ & $\mathcal{K}, \mathfrak{K}_{2}$ \\
\hline 25 & 34 & $2 \times 17$ & $1 b$ & $5^{2} 2^{4} 17^{4}$ & 3 & 0 & 0 & 1 & 6 & $(\times,-,-,-)$, & $\gamma$ & \\
\hline 26 & $\star 35$ & $5 \times 7$ & $1 \mathrm{a}$ & $5^{6} 7^{4}$ & 4 & 0 & 0 & 1 & 6 & $(-,-,-,, \otimes)$ & $\eta$ & \\
\hline 27 & 37 & & $1 \mathrm{~b}$ & $5^{2} 37^{4}$ & 1 & 0 & 0 & 0 & 5 & $(\times,-,-,-)$, & $\varepsilon$ & \\
\hline 28 & $* 38$ & $2 \times 19$ & $1 b$ & $5^{2} 2^{4} 19^{4}$ & 3 & 1 & 1 & 3 & 4 & $(-, \otimes,-,-)$ & $\beta_{2}$ & $5, \mathcal{K}$ \\
\hline 29 & 39 & $3 \times 13$ & $1 \mathrm{~b}$ & $5^{2} 3^{4} 13^{4}$ & 3 & 0 & 0 & 1 & 6 & $(\times,-,-,-)$ & $\gamma$ & \\
\hline 30 & 40 & $2^{3} \times 5$ & $1 \mathrm{a}$ & $5^{6} 2^{4}$ & 4 & 0 & 0 & 0 & 5 & $(\times,-,-,-)$ & $\varepsilon$ & \\
\hline 31 & 41 & & $1 \mathrm{~b}$ & $5^{2} 41^{4}$ & 1 & 1 & 1 & 2 & 3 & $(-,-, \otimes,-)$ & $\alpha_{2}$ & $\mathcal{L}, \mathfrak{K}_{2}$ \\
\hline 32 & ${ }^{*} 42$ & $2 \times 3 \times 7$ & $1 b$ & $5^{2} 2^{4} 3^{4} 7^{4}$ & 12 & 1 & 2 & 5 & 6 & $(\times,-,-,-)$ & $\gamma$ & $2 \times 5,3 \times 5^{2}$ \\
\hline 33 & 43 & & 2 & $43^{4}$ & 1 & 0 & 0 & 0 & 5 & $(-,-,-, \otimes)$ & $\vartheta$ & \\
\hline 34 & 44 & $2^{2} \times 11$ & $1 \mathrm{~b}$ & $5^{2} 2^{4} 11^{4}$ & 3 & 1 & 1 & 3 & 4 & $(-,-,(\times),-)$ & $\beta_{2}$ & $2 \times 5, \mathcal{K}$ \\
\hline 35 & 45 & $3^{2} \times 5$ & $1 \mathrm{a}$ & $5^{6} 3^{4}$ & 4 & 0 & 0 & 0 & 5 & $(\times,-,-,-)$ & $\varepsilon$ & \\
\hline 36 & 46 & $2 \times 23$ & $1 b$ & $5^{2} 2^{4} 23^{4}$ & 3 & 0 & 0 & 1 & 6 & $(\times,-,-,-)$, & $\gamma$ & \\
\hline 37 & 47 & & $1 \mathrm{~b}$ & $5^{2} 47^{4}$ & 1 & 0 & 0 & 0 & 5 & $(\times,-,-,--)$ & $\varepsilon$ & \\
\hline 38 & 48 & $2^{4} \times 3$ & $1 \mathrm{~b}$ & $5^{2} 2^{4} 3^{4}$ & 3 & 0 & 0 & 1 & 6 & $(\times,-,-,-)$ & $\gamma$ & \\
\hline 39 & 51 & $3 \times 17$ & 2 & $3^{4} 17^{4}$ & 1 & 0 & 0 & 0 & 5 & $(\times,-,-,-)$ & $\varepsilon$ & \\
\hline 40 & 52 & $2^{2} \times 13$ & $1 b$ & $5^{2} 2^{4} 13^{4}$ & 3 & 0 & 0 & 1 & 6 & $(\times,-,-,-)$, & $\gamma$ & \\
\hline
\end{tabular}


Table 7. 45 pure metacyclic fields with normalized radicands $53 \leq D \leq 104$.

\begin{tabular}{|c|c|c|c|c|c|c|c|c|c|c|c|c|}
\hline No. & $D$ & Factors & $S$ & $f^{*}$ & $m$ & $V_{L}$ & $V_{M}$ & $V_{N}$ & $E$ & $(1,2,4,5)$ & $\mathrm{T}$ & $\mathrm{P}$ \\
\hline 41 & 53 & & $1 \mathrm{~b}$ & $5^{2} 53^{4}$ & 1 & 0 & 0 & 0 & 2 & $(\times,-,-,-)$ & $\varepsilon$ & \\
\hline 42 & $\star 55$ & $5 \times 11$ & 1a & $5^{6} 11^{4}$ & 4 & 1 & 1 & 2 & 3 & $(-,-, \otimes,-)$ & $\alpha_{2}$ & $\mathcal{K}, \mathfrak{K}_{1}$ \\
\hline 43 & 56 & $2^{3} \times 7$ & $1 \mathrm{~b}$ & $5^{2} 2^{4} 7^{4}$ & 4 & 0 & 0 & 1 & 6 & $(\times,-,-,-)$ & $\gamma$ & \\
\hline 44 & $\star 57$ & $3 \times 19$ & 2 & $3^{4} 19^{4}$ & 1 & 1 & 1 & 2 & 3 & $(-, \otimes,-,-)$ & $\delta_{2}$ & $\mathcal{K}$ \\
\hline 45 & 58 & $2 \times 29$ & $1 b$ & $5^{2} 2^{4} 29^{4}$ & 3 & 1 & 1 & 3 & 4 & $(-, \otimes,-,-)$ & $\beta_{2}$ & $29 \times 5^{2}, \mathcal{K}$ \\
\hline 46 & 59 & & $1 \mathrm{~b}$ & $5^{2} 59^{4}$ & 1 & 1 & 1 & 2 & 3 & $(-, \otimes,-,-)$ & $\delta_{2}$ & $\mathcal{L}$ \\
\hline 47 & 60 & $2^{2} \times 3 \times 5$ & la & $5^{6} 2^{4} 3^{4}$ & 16 & 0 & 0 & 1 & 6 & $(\times,-,-,-)$ & $\gamma$ & \\
\hline 48 & 61 & & $1 \mathrm{~b}$ & $5^{2} 61^{4}$ & 1 & 1 & 1 & 2 & 3 & $(-,-, \otimes,-)$ & $\alpha_{2}$ & $\mathcal{L}, \mathfrak{K}_{2}$ \\
\hline 49 & 62 & $2 \times 31$ & $1 \mathrm{~b}$ & $5^{2} 2^{4} 31^{4}$ & 3 & 1 & 1 & 3 & 4 & $(-,-,(\times),-)$ & $\beta_{2}$ & $5, \mathcal{K}$ \\
\hline 50 & 63 & $3^{2} \times 7$ & $1 b$ & $5^{2} 3^{4} 7^{4}$ & 4 & 0 & 0 & 1 & 6 & $(\times,-,-,-)$ & $\gamma$ & \\
\hline 51 & 65 & $5 \times 13$ & 1a & $5^{6} 13^{4}$ & 4 & 0 & 0 & 0 & 5 & $(\times,-,-,-)$ & $\varepsilon$ & \\
\hline 52 & *66 & $2 \times 3 \times 11$ & $1 b$ & $5^{2} 2^{4} 3^{4} 11^{4}$ & 13 & 1 & 2 & 5 & 6 & $(-,-, \times,-)$ & $\gamma$ & $2 \times 5,3 \times 5^{3}$ \\
\hline 53 & 67 & & $1 b$ & $5^{2} 67^{4}$ & 1 & 0 & 0 & 0 & 5 & $(\times,-,-,-)$ & $\varepsilon$ & \\
\hline 54 & 68 & $2^{2} \times 17$ & 2 & $2^{4} 17^{4}$ & 1 & 0 & 0 & 0 & 5 & $(\times,-,-,-)$ & $\varepsilon$ & \\
\hline 55 & 69 & $3 \times 23$ & $1 b$ & $5^{2} 3^{4} 23^{4}$ & 3 & 0 & 0 & 1 & 6 & $(\times,-,-,-)$ & $\gamma$ & \\
\hline 56 & ${ }^{\star} 70$ & $2 \times 5 \times 7$ & la & $5^{6} 2^{4} 7^{4}$ & 16 & 0 & 0 & 1 & 6 & $(\times,-,-,-)$ & $\gamma$ & \\
\hline 57 & 71 & & $1 \mathrm{~b}$ & $5^{2} 71^{4}$ & 1 & 1 & 1 & 2 & 3 & $(-,-, \otimes,-)$ & $\alpha_{2}$ & $\mathcal{L}, \mathfrak{K}_{1}$ \\
\hline 58 & 73 & & $1 \mathrm{~b}$ & $5^{2} 73^{4}$ & 1 & 0 & 0 & 0 & 5 & $(\times,-,-,-)$ & $\varepsilon$ & \\
\hline 59 & 74 & $2 \times 37$ & 2 & $2^{4} 37^{4}$ & 1 & 0 & 0 & 0 & 5 & $(\times,-,-,-)$ & $\varepsilon$ & \\
\hline 60 & 75 & $3 \times 5^{2}$ & $1 \mathrm{a}$ & $5^{6} 3^{4}$ & 4 & 0 & 0 & 0 & 5 & $(\times,-,-,-)$ & $\varepsilon$ & \\
\hline 61 & 76 & $2^{2} \times 19$ & 2 & $2^{4} 19^{4}$ & 1 & 1 & 1 & 2 & 3 & $(-, \otimes,-,-)$ & $\delta_{2}$ & $\mathcal{K}$ \\
\hline 62 & *77 & $7 \times 11$ & $1 b$ & $5^{2} 7^{4} 11^{4}$ & 4 & 1 & 1 & 3 & 4 & $(-,-,(\times),-)$ & $\beta_{2}$ & $11 \times 5^{3}, \mathcal{K}$ \\
\hline 63 & $\star 78$ & $2 \times 3 \times 13$ & $1 \mathrm{~b}$ & $5^{2} 2^{4} 3^{4} 13^{4}$ & 13 & 1 & 2 & 5 & 6 & $(\times,-,-,-)$ & $\gamma$ & $3,2 \times 5^{3}$ \\
\hline 64 & 79 & & $1 \mathrm{~b}$ & $5^{2} 79^{4}$ & 1 & 1 & 1 & 2 & 3 & $(-, \otimes,-,-)$ & $\delta_{2}$ & $\mathcal{L}$ \\
\hline 65 & 80 & $2^{4} \times 5$ & 1a & $5^{6} 2^{4}$ & 4 & 0 & 0 & 0 & 5 & $(\times,-,-,-)$ & $\varepsilon$ & \\
\hline 66 & ${ }^{*} 82$ & $2 \times 41$ & 2 & $2^{4} 41^{4}$ & 1 & 1 & 1 & 2 & 3 & $(-,-, \otimes,-)$ & $\alpha_{2}$ & $\mathcal{K}, \mathfrak{K}_{2}$ \\
\hline 67 & 83 & & $1 \mathrm{~b}$ & $5^{2} 83^{4}$ & 1 & 0 & 0 & 0 & 5 & $(\times,-,-,-)$ & $\varepsilon$ & \\
\hline 68 & 84 & $2^{2} \times 3 \times 7$ & $1 \mathrm{~b}$ & $5^{2} 2^{4} 3^{4} 7^{4}$ & 12 & 1 & 2 & 5 & 6 & $(\times,-,-,-)$ & $\gamma$ & $2 \times 5,3 \times 5$ \\
\hline 69 & 85 & $5 \times 17$ & 1a & $5^{6} 17^{4}$ & 4 & 0 & 0 & 0 & 5 & $(\times,-,-,-)$, & $\varepsilon$ & \\
\hline 70 & 86 & $2 \times 43$ & $1 b$ & $5^{2} 2^{4} 43^{4}$ & 4 & 0 & 0 & 1 & 6 & $(\times,-,-,-)$ & $\gamma$ & \\
\hline 71 & 87 & $3 \times 29$ & $1 \mathrm{~b}$ & $5^{2} 3^{4} 29^{4}$ & 3 & 1 & 1 & 3 & 4 & $(-, \otimes,-,-)$ & $\beta_{2}$ & $29, \mathcal{L}$ \\
\hline 72 & 88 & $2^{3} \times 11$ & $1 \mathrm{~b}$ & $5^{2} 2^{4} 11^{4}$ & 3 & 2 & 2 & 4 & 1 & $(-,-, \otimes,-)$ & $\alpha_{2}$ & $\mathcal{K}, \mathfrak{K}_{2}$ \\
\hline 73 & 89 & & $1 \mathrm{~b}$ & $5^{2} 89^{4}$ & 1 & 1 & 1 & 2 & 3 & $(-, \otimes,-,-)$ & $\delta_{2}$ & $\mathcal{L}$ \\
\hline 74 & 90 & $2 \times 3^{2} \times 5$ & la & $5^{6} 2^{4} 3^{4}$ & 16 & 0 & 0 & 1 & 6 & $(\times,-,-,-)$ & $\gamma$ & \\
\hline 75 & 91 & $7 \times 13$ & $1 b$ & $5^{2} 7^{4} 13^{4}$ & 4 & 0 & 0 & 1 & 6 & $(\times,-,-,-)$ & $\gamma$ & \\
\hline 76 & 92 & $2^{2} \times 23$ & $1 \mathrm{~b}$ & $5^{2} 2^{4} 23^{4}$ & 3 & 0 & 0 & 1 & 6 & $(\times,-,-,-)$ & $\gamma$ & \\
\hline 77 & 93 & $3 \times 31$ & 2 & $3^{4} 31^{4}$ & 1 & 1 & 1 & 2 & 3 & $(-,-, \otimes,-)$ & $\alpha_{2}$ & $\mathcal{K}, \mathfrak{K}_{1}$ \\
\hline 78 & 94 & $2 \times 47$ & $1 \mathrm{~b}$ & $5^{2} 2^{4} 47^{4}$ & 3 & 0 & 0 & 1 & 6 & $(\times,-,-,-)$ & $\gamma$ & \\
\hline 79 & *95 & $5 \times 19$ & la & $5^{6} 19^{4}$ & 4 & 1 & 1 & 2 & 3 & $(-, \otimes,-,-)$ & $\delta_{2}$ & $\mathcal{K}$ \\
\hline 80 & 97 & & $1 \mathrm{~b}$ & $5^{2} 97^{4}$ & 1 & 0 & 0 & 0 & 5 & $(\times,-,-,-)$ & $\varepsilon$ & \\
\hline 81 & 99 & $3^{2} \times 11$ & 2 & $3^{4} 11^{4}$ & 1 & 1 & 1 & 2 & 3 & $(-,-, \otimes,-)$ & $\alpha_{2}$ & $\mathcal{K}, \mathfrak{K}_{1}$ \\
\hline 82 & ${ }^{\star} 101$ & & 2 & $101^{4}$ & 1 & 1 & 2 & 4 & 5 & $(-,-, \otimes, \otimes)$ & $\zeta_{1}$ & $\mathfrak{K}_{2}$ \\
\hline 83 & 102 & $2 \times 3 \times 17$ & $1 \mathrm{~b}$ & $5^{2} 2^{4} 3^{4} 17^{4}$ & 13 & 1 & 2 & 5 & 6 & $(\times,-,-,-)$ & $\gamma$ & $2,3 \times 5$ \\
\hline 84 & 103 & & $1 \mathrm{~b}$ & $5^{2} 103^{4}$ & 1 & 0 & 0 & 0 & 5 & $(\times,-,-,-)$ & $\varepsilon$ & \\
\hline 85 & 104 & $2^{3} \times 13$ & $1 \mathrm{~b}$ & $5^{2} 2^{4} 13^{4}$ & 3 & 0 & 0 & 1 & 6 & $(\times,-,-,-)$ & $\gamma$ & \\
\hline
\end{tabular}


Table 8. 45 pure metacyclic fields with normalized radicands $105 \leq D \leq 155$.

\begin{tabular}{|c|c|c|c|c|c|c|c|c|c|c|c|c|}
\hline No. & $D$ & Factors & S & $f^{*}$ & $m$ & $V_{L}$ & $V_{M}$ & $V_{N}$ & $E$ & $(1,2,4,5)$ & $\mathrm{T}$ & $\mathrm{P}$ \\
\hline 86 & 105 & $3 \times 5 \times 7$ & $1 \mathrm{a}$ & $5^{6} 3^{4} 7^{4}$ & 16 & 0 & 0 & 1 & 6 & $(\times,-,-,-)$ & $\gamma$ & \\
\hline 87 & 106 & $2 \times 53$ & $1 b$ & $5^{2} 2^{4} 53^{4}$ & 3 & 0 & 0 & 1 & 6 & $(\times,-,-,-)$ & $\gamma$ & \\
\hline 88 & 107 & & 2 & $107^{4}$ & 1 & 0 & 0 & 0 & 5 & $(-,-,-, \otimes)$ & $\vartheta$ & \\
\hline 89 & 109 & & $1 b$ & $5^{2} 109^{4}$ & 1 & 1 & 1 & 2 & 3 & $(-, \otimes,-,-)$ & $\delta_{2}$ & $\mathcal{L}$ \\
\hline 90 & ${ }^{*} 110$ & $2 \times 5 \times 11$ & $1 \mathrm{a}$ & $5^{6} 2^{4} 11^{4}$ & 16 & 1 & 1 & 3 & 4 & $(-,-,(\times),-)$ & $\beta_{2}$ & $11, \mathcal{L}$ \\
\hline 91 & 111 & $3 \times 37$ & $1 b$ & $5^{2} 3^{4} 37^{4}$ & 3 & 0 & 0 & 1 & 6 & $(\times,-,-,-)$ & $\gamma$ & \\
\hline 92 & 112 & $2^{4} \times 7$ & $1 b$ & $5^{2} 2^{4} 7^{4}$ & 4 & 0 & 0 & 1 & 6 & $(\times,-,-,-)$ & $\gamma$ & \\
\hline 93 & 113 & & $1 b$ & $5^{2} 113^{4}$ & 1 & 0 & 0 & 0 & 5 & $(\times,-,-,-)$ & $\varepsilon$ & \\
\hline 94 & ${ }^{*} 114$ & $2 \times 3 \times 19$ & $1 b$ & $5^{2} 2^{4} 3^{4} 19^{4}$ & 13 & 1 & 2 & 5 & 6 & $(-, \times,-,-)$ & $\gamma$ & $2 \times 5^{3}, 3 \times 5^{3}$ \\
\hline 95 & 115 & $5 \times 23$ & $1 \mathrm{a}$ & $5^{6} 23^{4}$ & 4 & 0 & 0 & 0 & 5 & $(\times,-,-,-)$ & $\varepsilon$ & \\
\hline 96 & 116 & $2^{2} \times 29$ & $1 b$ & $5^{2} 2^{4} 29^{4}$ & 3 & 1 & 1 & 3 & 4 & $(-, \otimes,-,-)$ & $\beta_{2}$ & $29 \times 5, \mathcal{K}$ \\
\hline 97 & 117 & $3^{2} \times 13$ & $1 \mathrm{~b}$ & $5^{2} 3^{4} 13^{4}$ & 3 & 0 & 0 & 1 & 6 & $(\times,-,-,-)$ & $\gamma$ & \\
\hline 98 & 118 & $2 \times 59$ & 2 & $2^{4} 59^{4}$ & 1 & 1 & 1 & 2 & 3 & $(-, \otimes,-,-)$ & $\delta_{2}$ & $\mathcal{K}$ \\
\hline 99 & 119 & $7 \times 17$ & $1 b$ & $5^{2} 7^{4} 17^{4}$ & 4 & 0 & 0 & 1 & 6 & $(\times,-,-,-)$ & $\gamma$ & \\
\hline 100 & 120 & $2^{3} \times 3 \times 5$ & $1 \mathrm{a}$ & $5^{6} 2^{4} 3^{4}$ & 16 & 0 & 0 & 1 & 6 & $(\times,-,-,-)$ & $\gamma$ & \\
\hline 101 & 122 & $2 \times 61$ & $1 b$ & $5^{2} 2^{4} 61^{4}$ & 3 & 1 & 1 & 3 & 4 & $(-,-,(\times),-)$ & $\beta_{2}$ & $61 \times 5^{3}, \mathcal{K}$ \\
\hline 102 & ${ }^{*} 123$ & $3 \times 41$ & $1 b$ & $5^{2} 3^{4} 41^{4}$ & 3 & 2 & 3 & 6 & 3 & $(-,-, \otimes,-)$ & $\alpha_{2}$ & $\mathcal{K}, \mathfrak{K}_{2}$ \\
\hline 103 & 124 & $2^{2} \times 31$ & 2 & $2^{4} 31^{4}$ & 1 & 1 & 1 & 2 & 3 & $(-,-, \otimes,-)$ & $\alpha_{2}$ & $\mathcal{K}, \mathfrak{K}_{1}$ \\
\hline 104 & ${ }^{*} 126$ & $2 \times 3^{2} \times 7$ & 2 & $2^{4} 3^{4} 7^{4}$ & 4 & 0 & 0 & 1 & 6 & $(\times,-,-,-)$, & $\gamma$ & \\
\hline 105 & 127 & & $1 b$ & $5^{2} 127^{4}$ & 1 & 0 & 0 & 0 & 5 & $(\times,-,-,-)$ & $\varepsilon$ & \\
\hline 106 & 129 & $3 \times 43$ & $1 b$ & $5^{2} 3^{4} 43^{4}$ & 4 & 0 & 0 & 1 & 6 & $(\times,-,-,-)$ & $\gamma$ & \\
\hline 107 & 130 & $2 \times 5 \times 13$ & $1 \mathrm{a}$ & $5^{6} 2^{4} 13^{4}$ & 16 & 0 & 0 & 1 & 6 & $(\times,-,-,-)$ & $\gamma$ & \\
\hline 108 & ${ }^{\star} 131$ & & $1 b$ & $5^{2} 131^{4}$ & 1 & 2 & 2 & 4 & 1 & $(-,-, \otimes,-)$ & $\alpha_{2}$ & $\mathcal{L}, \mathfrak{K}_{2}$ \\
\hline 109 & ${ }^{*} 132$ & $2^{2} \times 3 \times 11$ & 2 & $2^{4} 3^{4} 11^{4}$ & 3 & 1 & 1 & 3 & 4 & $(-,-,(\times),-)$ & $\beta_{2}$ & $11, \mathcal{L}$ \\
\hline 110 & ${ }^{*} 133$ & $7 \times 19$ & $1 \mathrm{~b}$ & $5^{2} 7^{4} 19^{4}$ & 4 & 1 & 1 & 3 & 4 & $(-, \otimes,-,-)$ & $\beta_{2}$ & $7, \mathcal{L}$ \\
\hline 111 & 134 & $2 \times 67$ & $1 \mathrm{~b}$ & $5^{2} 2^{4} 67^{4}$ & 3 & 0 & 0 & 1 & 6 & $(\times,-,-,-)$ & $\gamma$ & \\
\hline 112 & 136 & $2^{3} \times 17$ & $1 \mathrm{~b}$ & $5^{2} 2^{4} 17^{4}$ & 3 & 0 & 0 & 1 & 6 & $(\times,-,-,-)$ & $\gamma$ & \\
\hline 113 & 137 & & $1 b$ & $5^{2} 137^{4}$ & 1 & 0 & 0 & 0 & 5 & $(\times,-,-,-)$, & $\varepsilon$ & \\
\hline 114 & 138 & $2 \times 3 \times 23$ & $1 b$ & $5^{2} 2^{4} 3^{4} 23^{4}$ & 13 & 1 & 2 & 5 & 6 & $(\times,-,-,-)$, & $\gamma$ & $3,2 \times 5^{2}$ \\
\hline 115 & ${ }^{*} 139$ & & $1 \mathrm{~b}$ & $5^{2} 139^{4}$ & 1 & 1 & 1 & 3 & 4 & $(-, \otimes,-,-)$ & $\beta_{2}$ & $5, \mathcal{L}$ \\
\hline 116 & ${ }^{*} 140$ & $2^{2} \times 5 \times 7$ & $1 \mathrm{a}$ & $5^{6} 2^{4} 7^{4}$ & 16 & 1 & 2 & 4 & 5 & $(\times,-,-,-)$ & $\varepsilon$ & 7 \\
\hline 117 & *141 & $3 \times 47$ & $1 b$ & $5^{2} 3^{4} 47^{4}$ & 3 & 1 & 2 & 4 & 5 & $(\times,-,-,-)$ & $\varepsilon$ & $47 \times 5$ \\
\hline 118 & 142 & $2 \times 71$ & $1 b$ & $5^{2} 2^{4} 71^{4}$ & 3 & 1 & 1 & 3 & 4 & $(-,-,(\times),-)$ & $\beta_{2}$ & $71 \times 5^{2}, \mathcal{K}$ \\
\hline 119 & 143 & $11 \times 13$ & 2 & $11^{4} 13^{4}$ & 1 & 1 & 1 & 2 & 3 & $(-,-, \otimes,-)$ & $\alpha_{2}$ & $\mathcal{K}, \mathfrak{K}_{1}$ \\
\hline 120 & 145 & $5 \times 29$ & $1 \mathrm{a}$ & $5^{6} 29^{4}$ & 4 & 1 & 1 & 2 & 3 & $(-, \otimes,-,-)$ & $\delta_{2}$ & $\mathcal{K}$ \\
\hline 121 & 146 & $2 \times 73$ & $1 b$ & $5^{2} 2^{4} 73^{4}$ & 3 & 0 & 0 & 1 & 6 & $(\times,-,-,-)$ & $\gamma$ & \\
\hline 122 & 147 & $3 \times 7^{2}$ & $1 b$ & $5^{2} 3^{4} 7^{4}$ & 4 & 0 & 0 & 1 & 6 & $(\times,-,-,-)$ & $\gamma$ & \\
\hline 123 & 148 & $2^{2} \times 37$ & $1 b$ & $5^{2} 2^{4} 37^{4}$ & 3 & 0 & 0 & 1 & 6 & $(\times,-,-,-)$ & $\gamma$ & \\
\hline 124 & ${ }^{*} 149$ & & 2 & $149^{4}$ & 1 & 1 & 1 & 2 & 3 & $(-, \otimes,-, \times)$ & $\delta_{2}$ & $\mathcal{L}$ \\
\hline
\end{tabular}




\section{Continued}

\begin{tabular}{ccccccccccccc}
\hline 125 & 150 & $2 \times 3 \times 5^{2}$ & $1 \mathrm{a}$ & $5^{6} 2^{4} 3^{4}$ & 16 & 0 & 0 & 1 & 6 & $(\times,-,-,-)$ & $\gamma$ & \\
126 & ${ }^{*} 151$ & & 2 & $151^{4}$ & 1 & 1 & 1 & 2 & 3 & $(-,-, \otimes, \times)$ & $\alpha_{2}$ & $\mathcal{L}, \mathfrak{K}_{1}$ \\
127 & 152 & $2^{3} \times 19$ & $1 \mathrm{~b}$ & $5^{2} 2^{4} 19^{4}$ & 3 & 1 & 1 & 3 & 4 & $(-, \otimes,-,-)$ & $\beta_{2}$ & $19 \times 5^{2}, \mathcal{K}$ \\
128 & 153 & $3^{2} \times 17$ & $1 \mathrm{~b}$ & $5^{2} 3^{4} 17^{4}$ & 3 & 0 & 0 & 1 & 6 & $(\times,-,-,-)$ & $\gamma$ & \\
129 & ${ }^{*} 154$ & $2 \times 7 \times 11$ & $1 \mathrm{~b}$ & $5^{2} 2^{4} 7^{4} 11^{4}$ & 12 & 2 & 3 & 7 & 4 & $(-,-,(\times),-)$ & $\beta_{2}$ & $2 \times 11^{2}, \mathcal{K}$ \\
130 & ${ }^{*} 155$ & $5 \times 31$ & $1 \mathrm{a}$ & $5^{6} 31^{4}$ & 4 & 2 & 3 & 5 & 2 & $(-,-, \otimes,-)$ & $\alpha_{1}$ & $\mathfrak{K}_{1}, \mathfrak{K}_{2}$ \\
\hline
\end{tabular}

Table 9. 45 pure metacyclic fields with normalized radicands $156 \leq D \leq 207$.

\begin{tabular}{|c|c|c|c|c|c|c|c|c|c|c|c|c|}
\hline No. & $D$ & Factors & $S$ & $P^{*}$ & $m$ & $V_{L}$ & $V_{M}$ & $V_{N}$ & $E$ & $(1,2,4,5)$ & $\mathrm{T}$ & $\mathrm{P}$ \\
\hline 131 & 156 & $2^{2} \times 3 \times 13$ & $1 \mathrm{~b}$ & $5^{2} 2^{4} 3^{4} 13^{4}$ & 13 & 1 & 2 & 5 & 6 & $(\times,-,-,-)$ & $\gamma$ & $3,13 \times 5^{2}$ \\
\hline 132 & 157 & & 2 & $157^{4}$ & 1 & 0 & 0 & 0 & 5 & $(-,-,-, \otimes)$ & $\vartheta$ & \\
\hline 133 & 158 & $2 \times 79$ & $1 \mathrm{~b}$ & $5^{2} 2^{4} 79^{4}$ & 3 & 1 & 1 & 3 & 4 & $(-, \otimes,-,-)$ & $\beta_{2}$ & $5, \mathcal{K}$ \\
\hline 134 & 159 & $3 \times 53$ & $1 b$ & $5^{2} 3^{4} 53^{4}$ & 3 & 1 & 2 & 4 & 5 & $(\times,-,-,-)$ & $\varepsilon$ & 5 \\
\hline 135 & 161 & $7 \times 23$ & $1 b$ & $5^{2} 7^{4} 23^{4}$ & 4 & 0 & 0 & 1 & 6 & $(\times,-,-,-)$ & $\gamma$ & \\
\hline 136 & 163 & & $1 b$ & $5^{2} 163^{4}$ & 1 & 0 & 0 & 0 & 5 & $(\times,-,-,-)$ & $\varepsilon$ & \\
\hline 137 & 164 & $2^{2} \times 41$ & $1 \mathrm{~b}$ & $5^{2} 2^{4} 41^{4}$ & 3 & 1 & 1 & 3 & 4 & $(-,-,(\times),-)$ & $\beta_{2}$ & $41 \times 5, \mathcal{K}$ \\
\hline 138 & 165 & $3 \times 5 \times 11$ & 1a & $5^{6} 3^{4} 11^{4}$ & 16 & 1 & 1 & 3 & 4 & $(-,-,(\times),-)$ & $\beta_{2}$ & $5 \times 11^{2}, \mathcal{K}$ \\
\hline 139 & 166 & $2 \times 83$ & $1 b$ & $5^{2} 2^{4} 83^{4}$ & 3 & 1 & 2 & 4 & 5 & $(\times,-,-,-)$ & $\varepsilon$ & 5 \\
\hline 140 & 167 & & $1 b$ & $5^{2} 167^{4}$ & 1 & 0 & 0 & 0 & 5 & $(\times,-,-,-)$ & $\varepsilon$ & \\
\hline 141 & 168 & $2^{3} \times 3 \times 7$ & 2 & $2^{4} 3^{4} 7^{4}$ & 4 & 0 & 0 & 1 & 6 & $(\times,-,-,-)$ & $\gamma$ & \\
\hline 142 & 170 & $2 \times 5 \times 17$ & 1a & $5^{6} 2^{4} 17^{4}$ & 16 & 0 & 0 & 1 & 6 & $(\times,-,-,-)$ & $\gamma$ & \\
\hline 143 & ${ }^{\star} 171$ & $3^{2} \times 19$ & $1 b$ & $5^{2} 3^{4} 19^{4}$ & 3 & 1 & 2 & 4 & 5 & $(-, \times,-,-)$ & $\varepsilon$ & $19 \times 5^{2}$ \\
\hline 144 & 172 & $2^{2} \times 43$ & $1 b$ & $5^{2} 2^{4} 43^{4}$ & 4 & 0 & 0 & 1 & 6 & $(\times,-,-,-)$ & $\gamma$ & \\
\hline 145 & 173 & & $1 b$ & $5^{2} 173^{4}$ & 1 & 0 & 0 & 0 & 5 & $(\times,-,-,-)$ & $\varepsilon$ & \\
\hline 146 & ${ }^{\star} 174$ & $2 \times 3 \times 29$ & 2 & $2^{4} 3^{4} 29^{4}$ & 3 & 1 & 1 & 3 & 4 & $(-, \otimes,-,-)$ & $\beta_{2}$ & $3^{2} \times 29, \mathcal{K}$ \\
\hline 147 & 175 & $5^{2} \times 7$ & 1a & $5^{6} 7^{4}$ & 4 & 0 & 0 & 1 & 6 & $(-,-,-, \otimes)$ & $\eta$ & \\
\hline 148 & 176 & $2^{4} \times 11$ & 2 & $2^{4} 11^{4}$ & 1 & 1 & 1 & 2 & 3 & $(-,-, \otimes,-)$ & $\alpha_{2}$ & $\mathcal{K}, \mathfrak{K}_{2}$ \\
\hline 149 & 177 & $3 \times 59$ & $1 b$ & $5^{2} 3^{4} 59^{4}$ & 3 & 1 & 1 & 3 & 4 & $(-, \otimes,-,-)$ & $\beta_{2}$ & $3, \mathcal{L}$ \\
\hline 150 & 178 & $2 \times 89$ & $1 b$ & $5^{2} 2^{4} 89^{4}$ & 3 & 1 & 2 & 4 & 5 & $(-, \times,-,-)$ & $\varepsilon$ & $89 \times 5^{2}$ \\
\hline 151 & 179 & & $1 b$ & $5^{2} 179^{4}$ & 1 & 1 & 1 & 2 & 3 & $(-, \otimes,-,-)$ & $\delta_{2}$ & $\mathcal{L}$ \\
\hline 152 & ${ }^{\star} 180$ & $2^{2} \times 3^{2} \times 5$ & $1 \mathrm{a}$ & $5^{6} 2^{4} 3^{4}$ & 16 & 1 & 2 & 4 & 5 & $(\times,-,-,-)$ & $\varepsilon$ & 3 \\
\hline 153 & 181 & & $1 b$ & $5^{2} 181^{4}$ & 1 & 2 & 2 & 4 & 1 & $(-,-, \otimes,--)$ & $\alpha_{2}$ & $\mathcal{L}, \mathfrak{K}_{2}$ \\
\hline 154 & ${ }^{\star} 182$ & $2 \times 7 \times 13$ & 2 & $2^{4} 7^{4} 13^{4}$ & 4 & 1 & 2 & 4 & 5 & $(\times,-,-,-)$ & $\varepsilon$ & 7 \\
\hline 155 & 183 & $3 \times 61$ & $1 b$ & $5^{2} 3^{4} 61^{4}$ & 3 & 1 & 1 & 3 & 4 & $(-,-,(\times),-)$ & $\beta_{2}$ & $61 \times 5^{3}, \mathcal{K}$ \\
\hline 156 & 184 & $2^{3} \times 23$ & $1 b$ & $5^{2} 2^{4} 23^{4}$ & 3 & 0 & 0 & 1 & 6 & $(\times,-,-,-)$ & $\gamma$ & \\
\hline 157 & 185 & $5 \times 37$ & 1a & $5^{6} 37^{4}$ & 4 & 0 & 0 & 0 & 5 & $(\times,-,-,-)$ & $\varepsilon$ & \\
\hline 158 & ${ }^{\star} 186$ & $2 \times 3 \times 31$ & $1 b$ & $5^{2} 2^{4} 3^{4} 31^{4}$ & 13 & 2 & 3 & 6 & 3 & $(-,-, \otimes,--)$ & $\beta_{1}$ & $5, \mathfrak{K}_{2}$ \\
\hline 159 & 187 & $11 \times 17$ & $1 b$ & $5^{2} 11^{4} 17^{4}$ & 3 & 1 & 1 & 3 & 4 & $(-,-,(\times),-)$ & $\beta_{2}$ & $11 \times 5, \mathcal{K}$ \\
\hline
\end{tabular}




\section{Continued}

\begin{tabular}{|c|c|c|c|c|c|c|c|c|c|c|c|c|}
\hline 160 & 188 & $2^{2} \times 47$ & $1 b$ & $5^{2} 2^{4} 47^{4}$ & 3 & 0 & 0 & 1 & 6 & $(\times,-,-,-)$ & $\gamma$ & \\
\hline 161 & ${ }^{\star} 190$ & $2 \times 5 \times 19$ & 1a & $5^{6} 2^{4} 19^{4}$ & 16 & 1 & 1 & 3 & 4 & $(-, \otimes,-,-)$ & $\beta_{2}$ & $5, \mathcal{K}$ \\
\hline 162 & ${ }^{\star} 191$ & & $1 b$ & $5^{2} 191^{4}$ & 1 & 1 & 2 & 4 & 5 & $(-,-, \otimes,-)$ & $\beta_{1}$ & $5, \mathfrak{K}_{1}$ \\
\hline 163 & 193 & & 2 & $193^{4}$ & 1 & 0 & 0 & 0 & 5 & $(-,-,-, \otimes)$ & $\vartheta$ & \\
\hline 164 & 194 & $2 \times 97$ & $1 b$ & $5^{2} 2^{4} 97^{4}$ & 3 & 1 & 2 & 4 & 5 & $(\times,-,-,-)$ & $\varepsilon$ & $97 \times 5^{2}$ \\
\hline 165 & 195 & $3 \times 5 \times 13$ & $1 \mathrm{a}$ & $5^{6} 3^{4} 13^{4}$ & 16 & 0 & 0 & 1 & 6 & $(\times,-,-,-)$ & $\gamma$ & \\
\hline 166 & 197 & & $1 b$ & $5^{2} 197^{4}$ & 1 & 0 & 0 & 0 & 5 & $(\times,-,-,-)$ & $\varepsilon$ & \\
\hline 167 & 198 & $2 \times 3^{2} \times 11$ & $1 b$ & $5^{2} 2^{4} 3^{4} 11^{4}$ & 13 & 1 & 2 & 5 & 6 & $(-,-, \times,-)$ & $\gamma$ & $3 \times 5^{3}, 11 \times 5^{3}$ \\
\hline 168 & 199 & & 2 & $199^{4}$ & 1 & 1 & 1 & 2 & 3 & $(-, \otimes,-, \times)$ & $\delta_{2}$ & $\mathcal{L}$ \\
\hline 169 & 201 & $3 \times 67$ & 2 & $3^{4} 67^{4}$ & 1 & 0 & 0 & 0 & 5 & $(\times,-,-,-)$ & $\varepsilon$ & \\
\hline 170 & $\star 202$ & $2 \times 101$ & $1 b$ & $5^{2} 2^{4} 101^{4}$ & 4 & 1 & 1 & 3 & 4 & $(-,-,(\times),-)$ & $\beta_{2}$ & $2 \times 5, \mathcal{K}$ \\
\hline 171 & $\star 203$ & $7 \times 29$ & $1 b$ & $5^{2} 7^{4} 29^{4}$ & 4 & 1 & 2 & 4 & 5 & $(-, \times,-,-)$ & $\varepsilon$ & $29 \times 5$ \\
\hline 172 & 204 & $2^{2} \times 3 \times 17$ & $1 b$ & $5^{2} 2^{4} 3^{4} 17^{4}$ & 13 & 1 & 2 & 5 & 6 & $(\times,-,-,-)$ & $\gamma$ & $3 \times 5^{4}, 17 \times 5^{3}$ \\
\hline 173 & 205 & $5 \times 41$ & $1 \mathrm{a}$ & $5^{6} 41^{4}$ & 4 & 1 & 1 & 2 & 3 & $(-,-, \otimes,-)$ & $\alpha_{2}$ & $\mathcal{K}, \mathfrak{K}_{2}$ \\
\hline 174 & 206 & $2 \times 103$ & $1 b$ & $5^{2} 2^{4} 103^{4}$ & 3 & 1 & 2 & 4 & 5 & $(\times,-,-,-)$ & $\varepsilon$ & $2 \times 5^{2}$ \\
\hline 175 & 207 & $3^{2} \times 23$ & 2 & $3^{4} 23^{4}$ & 1 & 0 & 0 & 0 & 5 & $(\times,-,-,-)$ & $\varepsilon$ & \\
\hline
\end{tabular}

Table 10. 45 pure metacyclic fields with normalized radicands $208 \leq D \leq 259$.

\begin{tabular}{ccccccccccccc}
\hline No. & $D$ & Factors & $\mathrm{S}$ & $f^{A}$ & $m$ & $V_{L}$ & $V_{M}$ & $V_{N}$ & $E$ & $(1,2,4,5)$ & $\mathrm{T}$ & $\mathrm{P}$ \\
\hline 176 & 208 & $2^{4} \times 13$ & lb & $5^{2} 2^{4} 13^{4}$ & 3 & 0 & 0 & 1 & 6 & $(\times,-,-,-)$ & $\gamma$ & \\
177 & $* 209$ & $11 \times 19$ & $1 \mathrm{~b}$ & $5^{2} 11^{4} 19^{4}$ & 3 & 2 & 3 & 7 & 4 & $(-, \otimes, \times,-)$ & $\beta_{2}$ & $11 \times 5^{2}, \mathcal{K}_{(19)}$ \\
178 & $* 210$ & $2 \times 3 \times 5 \times 7$ & $1 \mathrm{a}$ & $5^{6} 2^{4} 3^{4} 7^{4}$ & 64 & 1 & 2 & 5 & 6 & $(\times,-,-,-)$ & $\gamma$ & $7,3^{2} \times 5$ \\
179 & $* 211$ & & $1 \mathrm{~b}$ & $5^{2} 211^{4}$ & 1 & 3 & 5 & 9 & 2 & $(-,-, \otimes,-)$ & $\delta_{1}$ & $\mathfrak{K}_{2}$ \\
180 & 212 & $2^{2} \times 53$ & $1 \mathrm{~b}$ & $5^{2} 2^{4} 53^{4}$ & 3 & 0 & 0 & 1 & 6 & $(\times,-,-,-)$ & $\gamma$ & \\
181 & 213 & $3 \times 71$ & $1 \mathrm{~b}$ & $5^{2} 3^{4} 71^{4}$ & 3 & 1 & 1 & 3 & 4 & $(-,-,(\times),-)$ & $\beta_{2}$ & $3 \times 5^{3}, \mathcal{K}$ \\
182 & 214 & $2 \times 107$ & $1 \mathrm{~b}$ & $5^{2} 2^{4} 107^{4}$ & 4 & 0 & 0 & 1 & 6 & $(\times,-,-,-)$ & $\gamma$ & \\
183 & 215 & $5 \times 43$ & $1 \mathrm{a}$ & $5^{6} 43^{4}$ & 4 & 0 & 0 & 1 & 6 & $(-,-,-, \otimes)$ & $\eta$ & \\
184 & 217 & $7 \times 31$ & $1 \mathrm{~b}$ & $5^{2} 7^{4} 31^{4}$ & 4 & 1 & 1 & 3 & 4 & $(-,-,(\times),-)$ & $\beta_{2}$ & $5, \mathcal{K}$ \\
185 & $* 218$ & $2 \times 109$ & 2 & $2^{4} 109^{4}$ & 1 & 1 & 2 & 4 & 5 & $(-, \times,-,-)$ & $\varepsilon$ & 2 \\
186 & 219 & $3 \times 73$ & $1 \mathrm{~b}$ & $5^{2} 3^{4} 73^{4}$ & 3 & 0 & 0 & 1 & 6 & $(\times,-,-,-)$ & $\gamma$ & \\
187 & 220 & $22^{2} \times 5 \times 11$ & $1 \mathrm{a}$ & $5^{6} 2^{4} 11^{4}$ & 16 & 1 & 1 & 3 & 4 & $(-,-,(\times),-)$ & $\beta_{2}$ & $2 \times 5, \mathcal{K}$ \\
188 & 221 & $13 \times 17$ & $1 \mathrm{~b}$ & $5^{2} 13^{4} 17^{4}$ & 3 & 0 & 0 & 1 & 6 & $(\times,-,-,-)$ & $\gamma$ & \\
189 & 222 & $2 \times 3 \times 37$ & $1 \mathrm{~b}$ & $5^{2} 2^{4} 3^{4} 37^{4}$ & 13 & 1 & 2 & 5 & 6 & $(\times,-,-,-)$ & $\gamma$ & $37,2 \times 5^{2}$ \\
190 & 223 & & $1 \mathrm{~b}$ & $5^{2} 223^{4}$ & 1 & 0 & 0 & 0 & 5 & $(\times,-,-,-)$ & $\varepsilon$ & \\
191 & 226 & $2 \times 113$ & 2 & $2^{4} 113^{4}$ & 1 & 0 & 0 & 0 & 5 & $(\times,-,-,-)$ & $\varepsilon$ & \\
192 & 227 & & $1 \mathrm{~b}$ & $5^{2} 227^{4}$ & 1 & 0 & 0 & 0 & 5 & $(\times,-,-,-)$ & $\varepsilon$ & \\
193 & 228 & $2^{2} \times 3 \times 19$ & $1 \mathrm{~b}$ & $5^{2} 2^{4} 3^{4} 19^{4}$ & 13 & 1 & 2 & 5 & 6 & $(-, \times,-,-)$ & $\gamma$ & $3 \times 5,19 \times 5$ \\
194 & 229 & & $1 \mathrm{~b}$ & $5^{2} 229^{4}$ & 1 & 1 & 1 & 2 & 3 & $(-, \otimes,-,-)$ & $\delta_{2}$ & $\mathcal{L}$ \\
\hline
\end{tabular}




\section{Continued}

\begin{tabular}{|c|c|c|c|c|c|c|c|c|c|c|c|c|}
\hline 195 & 230 & $2 \times 5 \times 23$ & $1 \mathrm{a}$ & $5^{6} 2^{4} 23^{4}$ & 16 & 0 & 0 & 1 & 6 & $(\times,-,-,-)$ & $\gamma$ & \\
\hline 196 & ${ }^{\star} 231$ & $3 \times 7 \times 11$ & $1 b$ & $5^{2} 3^{4} 7^{4} 11^{4}$ & 12 & 1 & 2 & 2 & 6 & $(-,-, \times,-)$ & $\gamma$ & $11,7 \times 5^{2}$ \\
\hline 197 & 232 & $2^{3} \times 29$ & 2 & $2^{4} 29^{4}$ & 1 & 1 & 1 & 2 & 3 & $(-, \otimes,-,-)$ & $\delta_{2}$ & $\mathcal{K}$ \\
\hline 198 & 233 & & $1 \mathrm{~b}$ & $5^{2} 233^{4}$ & 1 & 0 & 0 & 0 & 5 & $(\times,-,-,-)$ & $\varepsilon$ & \\
\hline 199 & 234 & $2 \times 3^{2} \times 13$ & $1 b$ & $5^{2} 2^{4} 3^{4} 13^{4}$ & 13 & 1 & 2 & 5 & 6 & $(\times,-,-,-)$ & $\gamma$ & $3,2 \times 5$ \\
\hline 200 & 235 & $5 \times 47$ & 1a & $5^{6} 47^{4}$ & 4 & 0 & 0 & 0 & 5 & $(\times,-,-,--)$ & $\varepsilon$ & \\
\hline 201 & 236 & $2^{2} \times 59$ & $1 \mathrm{~b}$ & $5^{2} 2^{4} 59^{4}$ & 3 & 1 & 1 & 3 & 4 & $(-, \otimes,-,-)$ & $\beta_{2}$ & $2 \times 5^{2}, \mathcal{K}$ \\
\hline 202 & 237 & $3 \times 79$ & $1 \mathrm{~b}$ & $5^{2} 3^{4} 79^{4}$ & 3 & 1 & 1 & 3 & 4 & $(-, \otimes,-,-)$ & $\beta_{2}$ & $3 \times 5, \mathcal{K}$ \\
\hline 203 & 238 & $2 \times 7 \times 17$ & $1 b$ & $5^{2} 2^{4} 7^{4} 17^{4}$ & 12 & 1 & 2 & 5 & 6 & $(\times,-,-,-)$ & $\gamma$ & $5,2^{4} \times 7$ \\
\hline 204 & 239 & & $1 \mathrm{~b}$ & $5^{2} 239^{4}$ & 1 & 1 & 1 & 2 & 3 & $(-, \otimes,-,-)$ & $\delta_{2}$ & $\mathcal{L}$ \\
\hline 205 & 240 & $2^{4} \times 3 \times 5$ & $1 \mathrm{a}$ & $5^{6} 2^{4} 3^{4}$ & 16 & 1 & 2 & 4 & 5 & $(\times,-,-,-)$ & $\varepsilon$ & 3 \\
\hline 206 & 241 & & $1 \mathrm{~b}$ & $5^{2} 241^{4}$ & 1 & 1 & 1 & 2 & 3 & $(-,-, \otimes,-)$ & $\alpha_{2}$ & $\mathcal{L}, \mathfrak{K}_{2}$ \\
\hline 207 & 244 & $2^{2} \times 61$ & $1 \mathrm{~b}$ & $5^{2} 2^{4} 61^{4}$ & 3 & 2 & 2 & 4 & 1 & $(-,-, \otimes,-)$ & $\alpha_{2}$ & $\mathcal{K}, \mathfrak{K}_{1}$ \\
\hline 208 & 245 & $5 \times 7^{2}$ & $1 \mathrm{a}$ & $5^{6} 7^{4}$ & 4 & 0 & 0 & 1 & 6 & $(-,-,-, \otimes)$ & $\eta$ & \\
\hline 209 & 246 & $2 \times 3 \times 41$ & $1 \mathrm{~b}$ & $5^{2} 2^{4} 3^{4} 41^{4}$ & 13 & 1 & 2 & 5 & 6 & $(-,-, \times,-)$ & $\gamma$ & $3,2 \cdot 5^{2}$ \\
\hline 210 & ${ }^{*} 247$ & $13 \times 19$ & $1 \mathrm{~b}$ & $5^{2} 13^{4} 19^{4}$ & 3 & 1 & 2 & 5 & 6 & $(-, \times,-,-)$ & $\gamma$ & \\
\hline 211 & 248 & $2^{3} \times 31$ & $1 \mathrm{~b}$ & $5^{2} 2^{4} 31^{4}$ & 3 & 1 & 1 & 3 & 4 & $(-,-,(\times),-)$ & $\beta_{2}$ & $5, \mathcal{K}$ \\
\hline 212 & 249 & $3 \times 83$ & 2 & $3^{4} 83^{4}$ & 1 & 0 & 0 & 0 & 5 & $(\times,-,-,-)$ & $\varepsilon$ & \\
\hline 213 & 251 & & 2 & $251^{4}$ & 1 & 1 & 1 & 2 & 3 & $(-,-, \otimes, \times)$ & $\alpha_{2}$ & $\mathcal{L}, \mathfrak{K}_{2}$ \\
\hline 214 & 252 & $2^{2} \times 3^{2} \times 7$ & $1 b$ & $5^{2} 2^{4} 3^{4} 7^{4}$ & 12 & 1 & 2 & 5 & 6 & $(\times,-,-,-)$ & $\gamma$ & $3,2 \times 5$ \\
\hline 215 & ${ }^{\star} 253$ & $11 \times 23$ & $1 \mathrm{~b}$ & $5^{2} 11^{4} 23^{4}$ & 3 & 1 & 2 & 4 & 5 & $(-,-, \otimes,-)$ & $\beta_{1}$ & $11, \mathfrak{K}_{1}$ \\
\hline 216 & 254 & $2 \times 127$ & $1 \mathrm{~b}$ & $5^{2} 2^{4} 127^{4}$ & 3 & 0 & 0 & 1 & 6 & $(\times,-,-,-)$ & $\gamma$ & \\
\hline 217 & 255 & $3 \times 5 \times 17$ & $1 \mathrm{a}$ & $5^{6} 3^{4} 17^{4}$ & 16 & 0 & 0 & 1 & 6 & $(\times,-,-,-)$ & $\gamma$ & \\
\hline 218 & 257 & & 2 & $257^{4}$ & 1 & 0 & 0 & 0 & 5 & $(-,-,-, \otimes)$ & $\vartheta$ & \\
\hline 219 & 258 & $2 \times 3 \times 43$ & $1 b$ & $5^{2} 2^{4} 3^{4} 43^{4}$ & 12 & 1 & 2 & 5 & 6 & $(\times,-,-,-)$ & $\gamma$ & $2,3 \times 5$ \\
\hline 220 & ${ }^{\star} 259$ & $7 \times 37$ & $1 \mathrm{~b}$ & $5^{2} 7^{4} 37^{4}$ & 4 & 1 & 2 & $5^{*}$ & 6 & $(\times,-,-,-)$ & $\gamma$ & \\
\hline
\end{tabular}

Table 11. 45 pure metacyclic fields with normalized radicands $260 \leq D \leq 307$.

\begin{tabular}{ccccccccccccc}
\hline No. & $D$ & Factors & $\mathrm{S}$ & $f^{\sharp}$ & $m$ & $V_{L}$ & $V_{M}$ & $V_{N}$ & $E$ & $(1,2,4,5)$ & $\mathrm{T}$ & $\mathrm{P}$ \\
\hline 221 & 260 & $2^{2} \times 5 \times 13$ & $1 \mathrm{a}$ & $5^{6} 2^{4} 13^{4}$ & 16 & 0 & 0 & 1 & 6 & $(\times,-,-,-)$ & $\gamma$ & \\
222 & 261 & $3^{2} \times 29$ & $1 \mathrm{~b}$ & $5^{2} 3^{4} 29^{4}$ & 3 & 1 & 1 & 3 & 4 & $(-, \otimes,-,-)$ & $\beta_{2}$ & $29, \mathcal{L}$ \\
223 & 262 & $2 \times 131$ & $1 \mathrm{~b}$ & $5^{2} 2^{4} 131^{4}$ & 3 & 1 & 1 & 3 & 4 & $(-,-,(\times),-)$ & $\beta_{2}$ & $131 \times 5, \mathcal{K}$ \\
224 & 263 & & $1 \mathrm{~b}$ & $5^{2} 263^{4}$ & 1 & 0 & 0 & 0 & 5 & $(\times,-,-,-)$ & $\varepsilon$ & \\
225 & 264 & $2^{3} \times 3 \times 11$ & $1 \mathrm{~b}$ & $5^{2} 2^{4} 3^{4} 11^{4}$ & 13 & 1 & 2 & 5 & 6 & $(-,-, \times,-)$ & $\gamma$ & $2 \times 5,2 \times 11$ \\
226 & 265 & $5 \times 53$ & $1 \mathrm{a}$ & $5^{6} 53^{4}$ & 4 & 0 & 0 & 0 & 5 & $(\times,-,-,-)$ & $\varepsilon$ & \\
227 & $\star 266$ & $2 \times 7 \times 19$ & $1 \mathrm{~b}$ & $5^{2} 2^{4} 7^{4} 19^{4}$ & 12 & 1 & 2 & 5 & 6 & $(-, \times,-,-)$ & $\gamma$ & $5,2^{3} \times 7$ \\
228 & 267 & $3 \times 89$ & $1 \mathrm{~b}$ & $5^{2} 3^{4} 89^{4}$ & 3 & 1 & 1 & 3 & 4 & $(-, \otimes,-,-)$ & $\beta_{2}$ & $89 \times 5^{2}, \mathcal{K}$ \\
229 & 268 & $2^{2} \times 67$ & 2 & $2^{4} 67^{4}$ & 1 & 0 & 0 & 0 & 5 & $(\times,-,-,-)$ & $\varepsilon$ & \\
\hline
\end{tabular}




\section{Continued}

\begin{tabular}{|c|c|c|c|c|c|c|c|c|c|c|c|c|}
\hline 230 & 269 & & $1 \mathrm{~b}$ & $5^{2} 269^{4}$ & 1 & 1 & 1 & 2 & 3 & $(-, \otimes,-,-)$ & $\delta_{2}$ & $\mathcal{L}$ \\
\hline 231 & 270 & $2 \times 3^{3} \times 5$ & $1 \mathrm{a}$ & $5^{6} 2^{4} 3^{4}$ & 16 & 0 & 0 & 1 & 6 & $(\times,-,-,-)$ & $\gamma$ & \\
\hline 232 & 271 & & $1 \mathrm{~b}$ & $5^{2} 271^{4}$ & 1 & 1 & 2 & 4 & 5 & $(-,-, \otimes,-)$ & $\beta_{1}$ & $5, \mathfrak{K}_{1}$ \\
\hline 233 & 272 & $2^{4} \times 17$ & $1 \mathrm{~b}$ & $5^{2} 2^{4} 17^{4}$ & 3 & 0 & 0 & 1 & 6 & $(\times,-,-,-)$ & $\gamma$ & \\
\hline 234 & $\star 273$ & $3 \times 7 \times 13$ & $1 \mathrm{~b}$ & $5^{2} 3^{4} 7^{4} 13^{4}$ & 12 & 2 & 4 & 8 & 5 & $(\times,-,-,-)$ & $\varepsilon$ & $7 \times 13^{2} \times 5^{4}$ \\
\hline 235 & 274 & $2 \times 137$ & 2 & $2^{4} 137^{4}$ & 1 & 0 & 0 & 0 & 5 & $(\times,-,-,--)$ & $\varepsilon$ & \\
\hline 236 & $\star 275$ & $5^{2} \times 11$ & $1 \mathrm{a}$ & $5^{6} 11^{4}$ & 4 & 2 & 2 & 4 & 1 & $(-,-, \otimes,-)$ & $\alpha_{2}$ & $\mathcal{K}, \mathfrak{K}_{2}$ \\
\hline 237 & $\star 276$ & $2^{2} \times 3 \times 23$ & 2 & $2^{4} 3^{4} 23^{4}$ & 3 & 0 & 0 & 1 & 6 & $(\times,-,-,-)$ & $\gamma$ & \\
\hline 238 & 277 & & $1 \mathrm{~b}$ & $5^{2} 277^{4}$ & 1 & 0 & 0 & 0 & 5 & $(\times,-,-,--)$ & $\varepsilon$ & \\
\hline 239 & 278 & $2 \times 139$ & $1 \mathrm{~b}$ & $5^{2} 2^{4} 139^{4}$ & 3 & 1 & 1 & 3 & 4 & $(-, \otimes,-,-)$ & $\beta_{2}$ & $139 \times 5, \mathcal{K}$ \\
\hline 240 & 279 & $3^{2} \times 31$ & $1 \mathrm{~b}$ & $5^{2} 3^{4} 31^{4}$ & 3 & 1 & 1 & 3 & 4 & $(-,-,(\times),-)$ & $\beta_{2}$ & $5, \mathcal{K}$ \\
\hline 241 & 280 & $2^{3} \times 5 \times 7$ & 1a & $5^{6} 2^{4} 7^{4}$ & 16 & 0 & 0 & 1 & 6 & $(\times,-,-,--)$ & $\gamma$ & \\
\hline 242 & ${ }^{*} 281$ & & $1 \mathrm{~b}$ & $5^{2} 281^{4}$ & 1 & 3 & $5^{*}$ & $9^{*}$ & 2 & $(-,-, \otimes,-)$ & $\alpha_{1}$ & $\mathfrak{K}_{1}, \mathfrak{K}_{2}$ \\
\hline 243 & 282 & $2 \times 3 \times 47$ & 2 & $2^{4} 3^{4} 47^{4}$ & 3 & 0 & 0 & 1 & 6 & $(\times,-,-,-)$ & $\gamma$ & \\
\hline 244 & 283 & & $1 \mathrm{~b}$ & $5^{2} 283^{4}$ & 1 & 0 & 0 & 0 & 5 & $(\times,-,-,-)$ & $\varepsilon$ & \\
\hline 245 & 284 & $2^{2} \times 71$ & $1 \mathrm{~b}$ & $5^{2} 2^{4} 71^{4}$ & 3 & 1 & 1 & 3 & 4 & $(-,-,(\times),-)$ & $\beta_{2}$ & $2 \times 5^{3}, \mathcal{K}$ \\
\hline 246 & $\star 285$ & $3 \times 5 \times 19$ & 1a & $5^{6} 3^{4} 19^{4}$ & 16 & 1 & 2 & 5 & 6 & $(-, \times,-,-)$ & $\gamma$ & \\
\hline 247 & $\star 286$ & $2 \times 11 \times 13$ & $1 \mathrm{~b}$ & $5^{2} 2^{4} 11^{4} 13^{4}$ & 13 & 2 & 3 & 7 & 4 & $(-,-,(\times),-)$ & $\beta_{2}$ & $11^{2} \times 5, \mathcal{K}$ \\
\hline 248 & ${ }^{*} 287$ & $7 \times 41$ & $1 \mathrm{~b}$ & $5^{2} 7^{4} 41^{4}$ & 4 & 2 & 2 & 4 & 1 & $(-,-, \otimes,-)$ & $\alpha_{2}$ & $\mathcal{K}, \mathfrak{K}_{1}$ \\
\hline 249 & $\star 290$ & $2 \times 5 \times 29$ & 1a & $5^{6} 2^{4} 29^{4}$ & 16 & 1 & 2 & 4 & 5 & $(-, \times,-,-)$ & $\varepsilon$ & 29 \\
\hline 250 & 291 & $3 \times 97$ & $1 \mathrm{~b}$ & $5^{2} 3^{4} 97^{4}$ & 3 & 0 & 0 & 1 & 6 & $(\times,-,-,-)$ & $\gamma$ & \\
\hline 251 & 292 & $2^{2} \times 73$ & $1 \mathrm{~b}$ & $5^{2} 2^{4} 73^{4}$ & 3 & 0 & 0 & 1 & 6 & $(\times,-,-,-)$ & $\gamma$ & \\
\hline 252 & 293 & & 2 & $293^{4}$ & 1 & 0 & 0 & 0 & 5 & $(-,-,-, \otimes)$ & $\vartheta$ & \\
\hline 253 & 294 & $2 \times 3 \times 7^{2}$ & $1 \mathrm{~b}$ & $5^{2} 2^{4} 3^{4} 7^{4}$ & 12 & 1 & 2 & 5 & 6 & $(\times,-,-,-)$ & $\gamma$ & $3,7^{2} \times 5$ \\
\hline 254 & 295 & $5 \times 59$ & $1 \mathrm{a}$ & $5^{6} 59^{4}$ & 4 & 1 & 1 & 2 & 3 & $(-, \otimes,-,-)$ & $\delta_{2}$ & $\mathcal{K}$ \\
\hline 255 & 296 & $2^{3} \times 37$ & $1 \mathrm{~b}$ & $5^{2} 2^{4} 37^{4}$ & 3 & 0 & 0 & 1 & 6 & $(\times,-,-,--)$ & $\gamma$ & \\
\hline 256 & 297 & $3^{3} \times 11$ & $1 \mathrm{~b}$ & $5^{2} 3^{4} 11^{4}$ & 3 & 1 & 1 & 3 & 4 & $(-,-,(\times),-)$ & $\beta_{2}$ & $11 \times 5, \mathcal{K}$ \\
\hline 257 & $\star 298$ & $2 \times 149$ & $1 \mathrm{~b}$ & $5^{2} 2^{4} 149^{4}$ & 4 & 1 & 2 & 4 & 5 & $(-, \times,-,-)$ & $\varepsilon$ & 5 \\
\hline 258 & 299 & $13 \times 23$ & 2 & $13^{4} 23^{4}$ & 1 & 0 & 0 & 0 & 5 & $(\times,-,-,-)$ & $\varepsilon$ & \\
\hline 259 & $\star 301$ & $7 \times 43$ & 2 & $7^{4} 43^{4}$ & 4 & 0 & 0 & 1 & 6 & $(-,-,-, \otimes)$ & $\eta$ & \\
\hline 260 & $\star 302$ & $2 \times 151$ & $1 \mathrm{~b}$ & $5^{2} 2^{4} 151^{4}$ & 4 & 1 & 2 & 4 & 5 & $(-,-, \otimes,-)$ & $\beta_{1}$ & $2, \mathfrak{K}_{1}$ \\
\hline 261 & 303 & $3 \times 101$ & $1 b$ & $5^{2} 3^{4} 101^{4}$ & 4 & 1 & 1 & 3 & 4 & $(-,-,(\times),-)$ & $\beta_{2}$ & $101 \times 5, \mathcal{K}$ \\
\hline 262 & 304 & $2^{4} \times 19$ & $1 \mathrm{~b}$ & $5^{2} 2^{4} 19^{4}$ & 3 & 1 & 1 & 3 & 4 & $(-, \otimes,-,-)$ & $\beta_{2}$ & $19 \times 5^{4}, \mathcal{K}$ \\
\hline 263 & 305 & $5 \times 61$ & $1 \mathrm{a}$ & $5^{6} 61^{4}$ & 4 & 2 & 2 & 4 & 1 & $(-,-, \otimes,-)$ & $\alpha_{2}$ & $\mathcal{K}, \mathfrak{K}_{1}$ \\
\hline 264 & 306 & $2 \times 3^{2} \times 17$ & $1 \mathrm{~b}$ & $5^{2} 2^{4} 3^{4} 17^{4}$ & 13 & 1 & 2 & 5 & 6 & $(\times,-,-,-)$ & $\gamma$ & $3 \times 5,3 \times 17$ \\
\hline 265 & 307 & & 2 & $307^{4}$ & 1 & 0 & 0 & 0 & 5 & $(-,-,-, \otimes)$ & $\vartheta$ & \\
\hline
\end{tabular}


Table 12. 45 pure metacyclic fields with normalized radicands $308 \leq D \leq 357$.

\begin{tabular}{|c|c|c|c|c|c|c|c|c|c|c|c|c|}
\hline No. & $D$ & Factors & $S$ & $f$ & $m$ & $V_{L}$ & $V_{M}$ & $V_{N}$ & $E$ & $(1,2,4,5)$ & $\mathrm{T}$ & $\mathrm{P}$ \\
\hline 266 & 308 & $2^{2} \times 7 \times 11$ & $1 \mathrm{~b}$ & $5^{2} 2^{4} 7^{4} 11^{4}$ & 12 & 1 & 2 & 5 & 6 & $(-,-, \times,-)$ & $\gamma$ & $11 \times 5,7 \times 5^{2}$ \\
\hline 267 & 309 & $3 \times 103$ & $1 \mathrm{~b}$ & $5^{2} 3^{4} 103^{4}$ & 3 & 1 & 2 & 4 & 5 & $(\times,-,-,-)$ & $\varepsilon$ & $103 \times 5^{2}$ \\
\hline 268 & 310 & $2 \times 5 \times 31$ & la & $5^{6} 2^{4} 31^{4}$ & 16 & 1 & 1 & 3 & 4 & $(-,-,(\times),-)$ & $\beta_{2}$ & $5, \mathcal{K}$ \\
\hline 269 & 311 & & $1 \mathrm{~b}$ & $5^{2} 311^{4}$ & 1 & 1 & 1 & 2 & 3 & $(-,-, \otimes,-)$ & $\alpha_{2}$ & $\mathcal{L}, \mathfrak{K}_{2}$ \\
\hline 270 & 312 & $2^{3} \times 3 \times 13$ & $1 \mathrm{~b}$ & $5^{2} 2^{4} 3^{4} 13^{4}$ & 13 & 1 & 2 & 5 & 6 & $(\times,-,-,-)$ & $\gamma$ & $5,2^{4} \times 13$ \\
\hline 271 & 313 & & $1 \mathrm{~b}$ & $5^{2} 313^{4}$ & 1 & 0 & 0 & 0 & 5 & $(\times,-,-,-)$ & $\varepsilon$ & \\
\hline 272 & 314 & $2 \times 157$ & $1 \mathrm{~b}$ & $5^{2} 2^{4} 157^{4}$ & 4 & 0 & 0 & 1 & 6 & $(\times,-,-,--)$ & $\gamma$ & \\
\hline 273 & 315 & $3^{2} \times 5 \times 7$ & 1a & $5^{6} 3^{4} 7^{4}$ & 16 & 1 & 2 & 4 & 5 & $(\times,-,-,-)$ & $\varepsilon$ & 7 \\
\hline 274 & 316 & $2^{2} \times 79$ & $1 \mathrm{~b}$ & $5^{2} 2^{4} 79^{4}$ & 3 & 1 & 1 & 3 & 4 & $(-, \otimes,-,-)$ & $\beta_{2}$ & $2 \times 5, \mathcal{K}$ \\
\hline 275 & 317 & & $1 b$ & $5^{2} 317^{4}$ & 1 & 0 & 0 & 0 & 5 & $(\times,-,-,-)$ & $\varepsilon$ & \\
\hline 276 & 318 & $2 \times 3 \times 53$ & 2 & $2^{4} 3^{4} 53^{4}$ & 3 & 0 & 0 & 1 & 6 & $(\times,-,-,-)$ & $\gamma$ & \\
\hline 277 & $\star 319$ & $11 \times 29$ & $1 \mathrm{~b}$ & $5^{2} 11^{4} 29^{4}$ & 3 & 2 & 2 & 5 & 2 & $(-, \otimes,(\times),-)$ & $\alpha_{3}$ & $\mathcal{K}_{(11)}, \mathcal{K}_{(29)}$ \\
\hline 278 & 321 & $3 \times 107$ & $1 b$ & $5^{2} 3^{4} 107^{4}$ & 4 & 0 & 0 & 1 & 6 & $(\times,-,-,-)$ & $\gamma$ & \\
\hline 279 & 322 & $2 \times 7 \times 23$ & $1 \mathrm{~b}$ & $5^{2} 2^{4} 7^{4} 23^{4}$ & 12 & 2 & 4 & 8 & 5 & $(\times,-,-,-)$ & $\varepsilon$ & $7 \times 23^{2} \times 5^{3}$ \\
\hline 280 & 323 & $17 \times 19$ & $1 b$ & $5^{2} 17^{4} 19^{4}$ & 3 & 1 & 1 & 3 & 4 & $(-, \otimes,-,-)$ & $\beta_{2}$ & $19 \times 5^{2}, \mathcal{K}$ \\
\hline 281 & 325 & $5^{2} \times 13$ & $1 \mathrm{a}$ & $5^{6} 13^{4}$ & 4 & 0 & 0 & 0 & 5 & $(\times,-,-,-)$ & $\varepsilon$ & \\
\hline 282 & 326 & $2 \times 163$ & 2 & $2^{4} 163^{4}$ & 1 & 0 & 0 & 0 & 5 & $(\times,-,-,--)$ & $\varepsilon$ & \\
\hline 283 & 327 & $3 \times 109$ & $1 \mathrm{~b}$ & $5^{2} 3^{4} 109^{4}$ & 3 & 1 & 2 & 5 & 6 & $(-, \times,-,-)$ & $\gamma$ & \\
\hline 284 & 328 & $2^{3} \times 41$ & $1 b$ & $5^{2} 2^{4} 41^{4}$ & 3 & 1 & 1 & 3 & 4 & $(-,-,(\times),-)$ & $\beta_{2}$ & $2 \times 41 \times 5, \mathcal{K}$ \\
\hline 285 & $* 329$ & $7 \times 47$ & $1 b$ & $5^{2} 7^{4} 47^{4}$ & 4 & 1 & 2 & 4 & 5 & $(\times,-,-,--)$ & $\varepsilon$ & 47 \\
\hline 286 & *330 & $2 \times 3 \times 5 \times 11$ & $1 \mathrm{a}$ & $5^{6} 2^{4} 3^{4} 11^{4}$ & 64 & 2 & 4 & 9 & 6 & $(-,-, \times,-)$ & $\gamma$ & $3 \times 11,5 \times 11^{3}$ \\
\hline 287 & 331 & & $1 \mathrm{~b}$ & $5^{2} 331^{4}$ & 1 & 1 & 1 & 2 & 3 & $(-,-, \otimes,-)$ & $\alpha_{2}$ & $\mathcal{L}, \mathfrak{K}_{1}$ \\
\hline 288 & 332 & $2^{2} \times 83$ & 2 & $2^{4} 83^{4}$ & 1 & 0 & 0 & 0 & 5 & $(\times,-,-,-)$ & $\varepsilon$ & \\
\hline 289 & 333 & $3^{2} \times 37$ & $1 b$ & $5^{2} 3^{4} 37^{4}$ & 3 & 0 & 0 & 1 & 6 & $(\times,-,-,--)$ & $\gamma$ & \\
\hline 290 & 334 & $2 \times 167$ & $1 \mathrm{~b}$ & $5^{2} 2^{4} 167^{4}$ & 3 & 0 & 0 & 1 & 6 & $(\times,-,-,-)$ & $\gamma$ & \\
\hline 291 & 335 & $5 \times 67$ & 1a & $5^{6} 67^{4}$ & 4 & 0 & 0 & 0 & 5 & $(\times,-,-,-)$ & $\varepsilon$ & \\
\hline 292 & 336 & $2^{4} \times 3 \times 7$ & $1 \mathrm{~b}$ & $5^{2} 2^{4} 3^{4} 7^{4}$ & 12 & 2 & 4 & 8 & 5 & $(\times,-,-,-)$ & $\varepsilon$ & $2 \times 5^{4}$ \\
\hline 293 & 337 & & $1 \mathrm{~b}$ & $5^{2} 337^{4}$ & 1 & 0 & 0 & 0 & 5 & $(\times,-,-,-)$ & $\varepsilon$ & \\
\hline 294 & 339 & $3 \times 113$ & $1 \mathrm{~b}$ & $5^{2} 3^{4} 113^{4}$ & 3 & 0 & 0 & 1 & 6 & $(\times,-,-,-)$ & $\gamma$ & \\
\hline 295 & 340 & $2^{2} \times 5 \times 17$ & $1 \mathrm{a}$ & $5^{6} 2^{4} 17^{4}$ & 16 & 0 & 0 & 1 & 6 & $(\times,-,-,-)$ & $\gamma$ & \\
\hline 296 & *341 & $11 \times 31$ & $1 \mathrm{~b}$ & $5^{2} 11^{4} 31^{4}$ & 3 & 3 & 5 & 9 & 2 & $(-,-, \otimes,-)$ & $\alpha_{1}$ & $\begin{array}{l}\mathfrak{K}_{(11), 1} \mathfrak{K}_{(31), 2}^{3}, \\
\mathfrak{K}_{(11), 2} \mathfrak{K}_{(31), 1}\end{array}$ \\
\hline 297 & 342 & $2 \times 3^{2} \times 19$ & $1 b$ & $5^{2} 2^{4} 3^{4} 19^{4}$ & 13 & 1 & 2 & 5 & 6 & $(-, \times,-,-)$ & $\gamma$ & $19,2 \times 5^{2}$ \\
\hline 298 & 344 & $2^{3} \times 43$ & $1 b$ & $5^{2} 2^{4} 43^{4}$ & 4 & 0 & 0 & 1 & 6 & $(\times,-,-,-)$ & $\gamma$ & \\
\hline 299 & 345 & $3 \times 5 \times 23$ & 1a & $5^{6} 3^{4} 23^{4}$ & 16 & 0 & 0 & 1 & 6 & $(\times,-,-,-)$ & $\gamma$ & \\
\hline 300 & 346 & $2 \times 173$ & $1 b$ & $5^{2} 2^{4} 173^{4}$ & 3 & 1 & 2 & 4 & 5 & $(\times,-,-,--)$ & $\varepsilon$ & $173 \times 5$ \\
\hline 301 & 347 & & $1 b$ & $5^{2} 347^{4}$ & 1 & 0 & 0 & 0 & 5 & $(\times,-,-,-)$ & $\varepsilon$ & \\
\hline 302 & ${ }^{*} 348$ & $2^{2} \times 3 \times 29$ & $1 \mathrm{~b}$ & $5^{2} 2^{4} 3^{4} 29^{4}$ & 13 & 2 & 4 & 8 & 5 & $(-, \times,-,-)$ & $\varepsilon$ & $2 \times 3 \times 5$ \\
\hline 303 & 349 & & 2 & $349^{4}$ & 1 & 1 & 1 & 2 & 3 & $(-, \otimes,-, \times)$ & $\delta_{2}$ & $\mathcal{L}$ \\
\hline
\end{tabular}




\section{Continued}

\begin{tabular}{ccccccccccccc}
\hline 304 & 350 & $2 \times 5^{2} \times 7$ & $1 \mathrm{a}$ & $5^{6} 2^{4} 7^{4}$ & 16 & 0 & 0 & 1 & 6 & $(\times,-,-,-)$ & $\gamma$ & \\
305 & 351 & $3^{3} \times 13$ & 2 & $3^{4} 13^{4}$ & 1 & 0 & 0 & 0 & 5 & $(\times,-,-,-)$ & $\varepsilon$ & \\
306 & 353 & & $1 \mathrm{~b}$ & $5^{2} 353^{4}$ & 1 & 0 & 0 & 0 & 5 & $(\times,-,-,-)$ & $\varepsilon$ & \\
307 & 354 & $2 \times 3 \times 59$ & $1 \mathrm{~b}$ & $5^{2} 2^{4} 3^{4} 59^{4}$ & 13 & 1 & 2 & 5 & 6 & $(-, \times,-,-)$ & $\gamma$ & $5,2 \times 3^{2}$ \\
308 & 355 & $5 \times 71$ & $1 \mathrm{a}$ & $5^{6} 71^{4}$ & 4 & 1 & 1 & 2 & 3 & $(-,-, \otimes,-)$ & $\alpha_{2}$ & $\mathcal{K}, \mathfrak{K}_{1}$ \\
309 & 356 & $2^{2} \times 89$ & $1 \mathrm{~b}$ & $5^{2} 2^{4} 89^{4}$ & 3 & 1 & 2 & 4 & 5 & $(-, \times,-,-)$ & $\varepsilon$ & $89 \times 5^{4}$ \\
310 & 357 & $3 \times 7 \times 17$ & 2 & $3^{4} 7^{4} 17^{4}$ & 4 & 0 & 0 & 1 & 6 & $(\times,-,-,-)$ & $\gamma$ & \\
\hline
\end{tabular}

Table 13. 45 pure metacyclic fields with normalized radicands $358 \leq D \leq 408$.

\begin{tabular}{|c|c|c|c|c|c|c|c|c|c|c|c|c|}
\hline No. & $D$ & Factors & $S$ & $f^{*}$ & $m$ & $V_{L}$ & $V_{M}$ & $V_{N}$ & $E$ & $(1,2,4,5)$ & $\mathrm{T}$ & $\mathrm{P}$ \\
\hline 311 & 358 & $2 \times 179$ & $1 b$ & $5^{2} 2^{4} 179^{4}$ & 3 & 1 & 1 & 3 & 4 & $(-, \otimes,-,-)$ & $\beta_{2}$ & $2, \mathcal{L}$ \\
\hline 312 & 359 & & $1 b$ & $5^{2} 359^{4}$ & 1 & 1 & 1 & 3 & 4 & $(-, \otimes,-,-)$ & $\beta_{2}$ & $5, \mathcal{L}$ \\
\hline 313 & 360 & $2^{3} \times 3^{2} \times 5$ & 1a & $5^{6} 2^{4} 3^{4}$ & 16 & 0 & 0 & 1 & 6 & $(\times,-,-,-)$ & $\gamma$ & \\
\hline 314 & 362 & $2 \times 181$ & $1 b$ & $5^{2} 2^{4} 181^{4}$ & 3 & 1 & 1 & 3 & 4 & $(-,-,(\times),-)$ & $\beta_{2}$ & $181 \times 5, \mathcal{K}$ \\
\hline 315 & 364 & $2^{2} \times 7 \times 13$ & $1 b$ & $5^{2} 2^{4} 7^{4} 13^{4}$ & 12 & 1 & 2 & 5 & 6 & $(\times,-,-,-)$ & $\gamma$ & $2,7 \times 13$ \\
\hline 316 & 365 & $5 \times 73$ & la & $5^{6} 73^{4}$ & 4 & 0 & 0 & 0 & 5 & $(\times,-,-,-)$ & $\varepsilon$ & \\
\hline 317 & 366 & $2 \times 3 \times 61$ & $1 \mathrm{~b}$ & $5^{2} 2^{4} 3^{4} 61^{4}$ & 13 & 2 & 3 & 6 & 3 & $(-,-, \otimes,-)$ & $\beta_{1}$ & $3 \times 61^{2} \times 5^{4}, \mathfrak{K}_{1}$ \\
\hline 318 & 367 & & $1 b$ & $5^{2} 367^{4}$ & 1 & 0 & 0 & 0 & 5 & $(\times,-,-,-)$ & $\varepsilon$ & \\
\hline 319 & 368 & $2^{4} \times 23$ & 2 & $2^{4} 23^{4}$ & 1 & 0 & 0 & 0 & 5 & $(\times,-,-,-)$ & $\varepsilon$ & \\
\hline 320 & 369 & $3^{2} \times 41$ & $1 \mathrm{~b}$ & $5^{2} 3^{4} 41^{4}$ & 3 & 2 & 3 & 6 & 3 & $(-,-, \otimes,-)$ & $\alpha_{2}$ & $\mathcal{K}, \mathfrak{K}_{2}$ \\
\hline 321 & 370 & $2 \times 5 \times 37$ & la & $5^{6} 2^{4} 37^{4}$ & 16 & 0 & 0 & 1 & 6 & $(\times,-,-,-)$ & $\gamma$ & \\
\hline 322 & 371 & $7 \times 53$ & $1 b$ & $5^{2} 7^{4} 53^{4}$ & 4 & 0 & 0 & 1 & 6 & $(\times,-,-,-)$ & $\gamma$ & \\
\hline 323 & 372 & $2^{2} \times 3 \times 31$ & $1 b$ & $5^{2} 2^{4} 3^{4} 31^{4}$ & 13 & 1 & 2 & 5 & 6 & $(-,-,(\times),-)$ & $\gamma$ & $5,2 \times 31$ \\
\hline 324 & 373 & & $1 \mathrm{~b}$ & $5^{2} 373^{4}$ & 1 & 0 & 0 & 0 & 5 & $(\times,-,-,-)$ & $\varepsilon$ & \\
\hline 325 & 374 & $2 \times 11 \times 17$ & 2 & $2^{4} 11^{4} 17^{4}$ & 3 & 1 & 1 & 3 & 4 & $(-,-,(\times),-)$ & $\beta_{2}$ & $11, \mathcal{L}$ \\
\hline 326 & 376 & $2^{3} \times 47$ & 2 & $2^{4} 47^{4}$ & 1 & 0 & 0 & 0 & 5 & $(\times,-,-,-)$ & $\varepsilon$ & \\
\hline 327 & *377 & $13 \times 29$ & $1 b$ & $5^{2} 13^{4} 29^{4}$ & 3 & 2 & 3 & 6 & 3 & $(-, \otimes,-,-)$ & $\delta_{2}$ & $\mathcal{K}$ \\
\hline 328 & 378 & $2 \times 3^{3} \times 7$ & $1 b$ & $5^{2} 2^{4} 3^{4} 7^{4}$ & 12 & 1 & 2 & 5 & 6 & $(\times,-,-,-)$ & $\gamma$ & $2 \times 5^{2}, 3^{3} \times 7^{2}$ \\
\hline 329 & $\star 379$ & & $1 b$ & $5^{2} 379^{4}$ & 1 & 1 & 2 & 4 & 5 & $(-, \times,-,-)$ & $\varepsilon$ & 5 \\
\hline 330 & 380 & $2^{2} \times 5 \times 19$ & la & $5^{6} 2^{4} 19^{4}$ & 16 & 1 & 1 & 3 & 4 & $(-, \otimes,-,-)$ & $\beta_{2}$ & $19, \mathcal{L}$ \\
\hline 331 & 381 & $3 \times 127$ & $1 b$ & $5^{2} 3^{4} 127^{4}$ & 3 & 0 & 0 & 1 & 6 & $(\times,-,-,-)$ & $\gamma$ & \\
\hline 332 & 382 & $2 \times 191$ & 2 & $2^{4} 191^{4}$ & 1 & 1 & 1 & 2 & 3 & $(-,-, \otimes,-)$ & $\alpha_{2}$ & $\mathcal{K}, \mathfrak{K}_{1}$ \\
\hline 333 & 383 & & $1 b$ & $5^{2} 383^{4}$ & 1 & 0 & 0 & 0 & 5 & $(\times,-,-,-)$ & $\varepsilon$ & \\
\hline 334 & *385 & $5 \times 7 \times 11$ & 1a & $5^{6} 7^{4} 11^{4}$ & 16 & 1 & 1 & 3 & 4 & $(-,-,(\times),-)$ & $\beta_{2}$ & $7 \times 11^{2}, \mathcal{K}$ \\
\hline 335 & 386 & $2 \times 193$ & $1 b$ & $5^{2} 2^{4} 193^{4}$ & 4 & 1 & 2 & 4 & 5 & $(\times,-,-,-)$ & $\varepsilon$ & 2 \\
\hline
\end{tabular}




\section{Continued}

\begin{tabular}{|c|c|c|c|c|c|c|c|c|c|c|c|c|}
\hline 336 & 387 & $3^{2} \times 43$ & $1 b$ & $5^{2} 3^{4} 43^{4}$ & 4 & 0 & 0 & 1 & 6 & $(\times,-,-,-)$ & $\gamma$ & \\
\hline 337 & 388 & $2^{2} \times 97$ & $1 b$ & $5^{2} 2^{4} 97^{4}$ & 3 & 1 & 2 & 4 & 5 & $(\times,-,-,-)$ & $\varepsilon$ & $97 \times 5^{4}$ \\
\hline 338 & 389 & & $1 b$ & $5^{2} 389^{4}$ & 1 & 1 & 1 & 2 & 3 & $(-, \otimes,-,-)$ & $\delta_{2}$ & $\mathcal{L}$ \\
\hline 339 & $\star 390$ & $2 \times 3 \times 5 \times 13$ & 1a & $5^{6} 2^{4} 3^{4} 13^{4}$ & 64 & 1 & 2 & 5 & 6 & $(\times,-,-,-)$ & $\gamma$ & 2,5 \\
\hline 340 & 391 & $17 \times 23$ & $1 b$ & $5^{2} 17^{4} 23^{4}$ & 3 & 0 & 0 & 1 & 6 & $(\times,-,-,-)$ & $\gamma$ & \\
\hline 341 & 393 & $3 \times 131$ & 2 & $3^{4} 131^{4}$ & 1 & 1 & 1 & 2 & 3 & $(-,-, \otimes,-)$ & $\alpha_{2}$ & $\mathcal{K}, \mathfrak{K}_{1}$ \\
\hline 342 & 394 & $2 \times 197$ & $1 b$ & $5^{2} 2^{4} 197^{4}$ & 3 & 0 & 0 & 1 & 6 & $(\times,-,-,-)$ & $\gamma$ & \\
\hline 343 & 395 & $5 \times 79$ & 1a & $5^{6} 79^{4}$ & 4 & 1 & 1 & 2 & 3 & $(-, \otimes,-,-)$ & $\delta_{2}$ & $\mathcal{K}$ \\
\hline 344 & 396 & $2^{2} \times 3^{2} \times 11$ & $1 b$ & $5^{2} 2^{4} 3^{4} 11^{4}$ & 13 & 2 & 3 & 6 & 3 & $(-,-, \otimes,-)$ & $\beta_{1}$ & $11 \times 5^{2}, \mathfrak{K}_{2}$ \\
\hline 345 & 397 & & $1 b$ & $5^{2} 397^{4}$ & 1 & 0 & 0 & 0 & 5 & $(\times,-,-,-)$ & $\varepsilon$ & \\
\hline 346 & $\star 398$ & $2 \times 199$ & $1 b$ & $5^{2} 2^{4} 199^{4}$ & 4 & 1 & 1 & 3 & 4 & $(-, \otimes,-,-)$ & $\beta_{2}$ & $5, \mathcal{K}$ \\
\hline 347 & *399 & $3 \times 7 \times 19$ & 2 & $3^{4} 7^{4} 19^{4}$ & 4 & 1 & 1 & 3 & 4 & $(-, \otimes,-,-)$ & $\beta_{2}$ & $3 \times 19^{2}, \mathcal{K}$ \\
\hline 348 & $* 401$ & & 2 & $401^{4}$ & 1 & 2 & 3 & 5 & 2 & $(-,-, \otimes, \times)$ & $\alpha_{1}$ & $\mathfrak{K}_{1}, \mathfrak{K}_{2}$ \\
\hline 349 & 402 & $2 \times 3 \times 67$ & $1 b$ & $5^{2} 2^{4} 3^{4} 67^{4}$ & 13 & 1 & 2 & 5 & 6 & $(\times,-,-,-)$ & $\gamma$ & $3 \times 5^{3}, 2 \times 5^{3}$ \\
\hline 350 & 403 & $13 \times 31$ & $1 b$ & $5^{2} 13^{4} 31^{4}$ & 3 & 1 & 1 & 3 & 4 & $(-,-,(\times),-)$ & $\beta_{2}$ & $5, \mathcal{K}$ \\
\hline 351 & 404 & $2^{2} \times 101$ & $1 b$ & $5^{2} 2^{4} 101^{4}$ & 4 & 1 & 1 & 3 & 4 & $(-,-,(\times),-)$ & $\beta_{2}$ & $2 \times 5, \mathcal{K}$ \\
\hline 352 & 405 & $3^{4} \times 5$ & $1 \mathrm{a}$ & $5^{6} 3^{4}$ & 4 & 0 & 0 & 0 & 5 & $(\times,-,-,-)$ & $\varepsilon$ & \\
\hline 353 & 406 & $2 \times 7 \times 29$ & $1 \mathrm{~b}$ & $5^{2} 2^{4} 7^{4} 29^{4}$ & 12 & 1 & 2 & 5 & 6 & $(-, \times,-,-)$ & $\gamma$ & $7,2 \times 5^{3}$ \\
\hline 354 & 407 & $11 \times 37$ & 2 & $11^{4} 37^{4}$ & 1 & 1 & 1 & 2 & 3 & $(-,-, \otimes,-)$ & $\alpha_{2}$ & $\mathcal{K}, \mathfrak{K}_{2}$ \\
\hline 355 & 408 & $2^{3} \times 3 \times 17$ & $1 b$ & $5^{2} 2^{4} 3^{4} 17^{4}$ & 13 & 1 & 2 & 5 & 6 & $(\times,-,-,-)$ & $\gamma$ & $17,3 \times 5^{3}$ \\
\hline
\end{tabular}

Table 14. 45 pure metacyclic fields with normalized radicands $409 \leq D \leq 458$.

\begin{tabular}{ccccccccccccc}
\hline No. & $D$ & Factors & $\mathrm{S}$ & $f^{f}$ & $m$ & $V_{L}$ & $V_{M}$ & $V_{N}$ & $E$ & $(1,2,4,5)$ & $\mathrm{T}$ & $\mathrm{P}$ \\
\hline 356 & 409 & & $1 \mathrm{~b}$ & $5^{2} 409^{4}$ & 1 & 1 & 1 & 2 & 3 & $(-, \otimes,-,-)$ & $\delta_{2}$ & $\mathcal{L}$ \\
357 & 410 & $2 \times 5 \times 41$ & $1 \mathrm{a}$ & $5^{6} 2^{4} 41^{4}$ & 16 & 1 & 1 & 3 & 4 & $(-,-,(\times),-)$ & $\beta_{2}$ & $2 \times 41^{2}, \mathcal{K}$ \\
358 & 411 & $3 \times 137$ & $1 \mathrm{~b}$ & $5^{2} 3^{4} 137^{4}$ & 3 & 0 & 0 & 1 & 6 & $(\times,-,-,-)$ & $\gamma$ & \\
359 & 412 & $2^{2} \times 103$ & $1 \mathrm{~b}$ & $5^{2} 2^{4} 103^{4}$ & 3 & 1 & 2 & 4 & 5 & $(\times,-,-,-)$ & $\varepsilon$ & $2 \times 5^{2}$ \\
360 & 413 & $7 \times 59$ & $1 \mathrm{~b}$ & $5^{2} 7^{4} 59^{4}$ & 4 & 1 & 1 & 3 & 4 & $(-, \otimes,-,-)$ & $\beta_{2}$ & $59 \times 5, \mathcal{K}$ \\
361 & 414 & $2 \times 3^{2} \times 23$ & $1 \mathrm{~b}$ & $5^{2} 2^{4} 3^{4} 23^{4}$ & 13 & 1 & 2 & 5 & 6 & $(\times,-,-,-)$ & $\gamma$ & 2,3 \\
362 & 415 & $5 \times 83$ & $1 \mathrm{a}$ & $5^{6} 83^{4}$ & 4 & 0 & 0 & 0 & 5 & $(\times,-,-,-)$ & $\varepsilon$ & \\
363 & 417 & $3 \times 139$ & $1 \mathrm{~b}$ & $5^{2} 3^{4} 139^{4}$ & 3 & 1 & 1 & 3 & 4 & $(-, \otimes,-,-)$ & $\beta_{2}$ & $139 \times 5, \mathcal{K}$ \\
364 & $* 418$ & $2 \times 11 \times 19$ & 2 & $2^{4} 11^{4} 19^{4}$ & 3 & 2 & 3 & 6 & 3 & $(-, \otimes, \otimes,-)$ & $\alpha_{2}$ & $\mathcal{K}_{(19)}, \mathfrak{K}_{(11), 1}$ \\
365 & 419 & & $1 \mathrm{~b}$ & $5^{2} 419^{4}$ & 1 & 1 & 1 & 3 & 4 & $(-, \otimes,-,-)$ & $\beta_{2}$ & $5, \mathcal{L}$ \\
366 & 420 & $2^{2} \times 3 \times 5 \times 7$ & $1 \mathrm{a}$ & $5^{6} 2^{4} 3^{4} 7^{4}$ & 64 & 1 & 2 & 5 & 6 & $(\times,-,-,-)$ & $\gamma$ & $2,3^{2} \times 7$ \\
\hline
\end{tabular}




\section{Continued}

\begin{tabular}{|c|c|c|c|c|c|c|c|c|c|c|c|c|}
\hline 367 & ${ }^{\star} 421$ & & $1 \mathrm{~b}$ & $5^{2} 421^{4}$ & 1 & 1 & 2 & 3 & 4 & $(-,-, \otimes,-)$ & $\delta_{1}$ & $\mathfrak{K}_{2}$ \\
\hline 368 & ${ }^{*} 422$ & $2 \times 211$ & $1 \mathrm{~b}$ & $5^{2} 2^{4} 211^{4}$ & 3 & 1 & 2 & 4 & 5 & $(-,-, \times,-)$ & $\varepsilon$ & $2 \times 5^{2}$ \\
\hline 369 & 423 & $3^{2} \times 47$ & $1 \mathrm{~b}$ & $5^{2} 3^{4} 47^{4}$ & 3 & 1 & 2 & 4 & 5 & $(\times,-,-,-)$ & $\varepsilon$ & $3 \times 5^{3}$ \\
\hline 370 & 424 & $2^{3} \times 53$ & 2 & $2^{4} 53^{4}$ & 1 & 0 & 0 & 0 & 5 & $(\times,-,-,-)$ & $\varepsilon$ & \\
\hline 371 & 425 & $5^{2} \times 17$ & 1a & $5^{6} 17^{4}$ & 4 & 0 & 0 & 0 & 5 & $(\times,-,-,-)$ & $\varepsilon$ & \\
\hline 372 & 426 & $2 \times 3 \times 71$ & 2 & $2^{4} 3^{4} 71^{4}$ & 3 & 1 & 1 & 3 & 4 & $(-,-,(\times),-)$ & $\beta_{2}$ & $2^{2} \times 71, \mathcal{K}$ \\
\hline 373 & 427 & $7 \times 61$ & $1 \mathrm{~b}$ & $5^{2} 7^{4} 61^{4}$ & 4 & 1 & 1 & 3 & 4 & $(-,-,(\times),-)$ & $\beta_{2}$ & $61 \times 5^{2}, \mathcal{K}$ \\
\hline 374 & 428 & $2^{2} \times 107$ & $1 \mathrm{~b}$ & $5^{2} 2^{4} 107^{4}$ & 4 & 0 & 0 & 1 & 6 & $(\times,-,-,-)$ & $\gamma$ & \\
\hline 375 & 429 & $3 \times 11 \times 13$ & $1 \mathrm{~b}$ & $5^{2} 3^{4} 11^{4} 13^{4}$ & 13 & 2 & 3 & 7 & 4 & $(-,-,(\times),-)$ & $\beta_{2}$ & $13 \times 5, \mathcal{K}$ \\
\hline 376 & 430 & $2 \times 5 \times 43$ & 1a & $5^{6} 2^{4} 43^{4}$ & 16 & 1 & 2 & 4 & 5 & $(\times,-,-,-)$ & $\varepsilon$ & 43 \\
\hline 377 & 431 & & $1 \mathrm{~b}$ & $5^{2} 431^{4}$ & 1 & 1 & 1 & 2 & 3 & $(-,-, \otimes,-)$ & $\alpha_{2}$ & $\mathcal{L}, \mathfrak{K}_{1}$ \\
\hline 378 & 433 & & $1 \mathrm{~b}$ & $5^{2} 433^{4}$ & 1 & 0 & 0 & 0 & 5 & $(\times,-,-,-)$, & $\varepsilon$ & \\
\hline 379 & 434 & $2 \times 7 \times 31$ & $1 \mathrm{~b}$ & $5^{2} 2^{4} 7^{4} 31^{4}$ & 12 & 1 & 2 & 5 & 6 & $(-,-, \times,-)$ & $\gamma$ & $5,2 \times 31^{2}$ \\
\hline 380 & 435 & $3 \times 5 \times 29$ & $1 \mathrm{a}$ & $5^{6} 3^{4} 29^{4}$ & 16 & 1 & 1 & 3 & 4 & $(-, \otimes,-,-)$ & $\beta_{2}$ & $3 \times 5^{3}, \mathcal{K}$ \\
\hline 381 & 436 & $2^{2} \times 109$ & $1 \mathrm{~b}$ & $5^{2} 2^{4} 109^{4}$ & 3 & 1 & 1 & 3 & 4 & $(-, \otimes,-,-)$ & $\beta_{2}$ & $109, \mathcal{L}$ \\
\hline 382 & 437 & $19 \times 23$ & $1 \mathrm{~b}$ & $5^{2} 19^{4} 23^{4}$ & 3 & 1 & 1 & 3 & 4 & $(-, \otimes,-,-)$ & $\beta_{2}$ & $19 \times 5^{2}, \mathcal{K}$ \\
\hline 383 & 438 & $2 \times 3 \times 73$ & $1 \mathrm{~b}$ & $5^{2} 2^{4} 3^{4} 73^{4}$ & 13 & 1 & 2 & 5 & 6 & $(\times,-,-,-)$ & $\gamma$ & 3,5 \\
\hline 384 & 439 & & $1 \mathrm{~b}$ & $5^{2} 439^{4}$ & 1 & 1 & 1 & 2 & 3 & $(-, \otimes,-,-)$ & $\delta_{2}$ & $\mathcal{L}$ \\
\hline 385 & 440 & $2^{3} \times 5 \times 11$ & $1 \mathrm{a}$ & $5^{6} 2^{4} 11^{4}$ & 16 & 1 & 1 & 3 & 4 & $(-,-,(\times),-)$ & $\beta_{2}$ & $2 \times 5, \mathcal{K}$ \\
\hline 386 & 442 & $2 \times 13 \times 17$ & $1 \mathrm{~b}$ & $5^{2} 2^{4} 13^{4} 17^{4}$ & 13 & 1 & 2 & 5 & 6 & $(\times,-,-,-)$ & $\gamma$ & $2 \times 5^{4}, 13 \times 5^{2}$ \\
\hline 387 & 443 & & 2 & $443^{4}$ & 1 & 0 & 0 & 0 & 5 & $(-,-,-, \otimes)$ & $\vartheta$ & \\
\hline 388 & 444 & $2^{2} \times 3 \times 37$ & $1 \mathrm{~b}$ & $5^{2} 2^{4} 3^{4} 37^{4}$ & 13 & 1 & 2 & 5 & 6 & $(\times,-,-,--)$ & $\gamma$ & $5,2^{3} \times 3$ \\
\hline 389 & 445 & $5 \times 89$ & $1 \mathrm{a}$ & $5^{6} 89^{4}$ & 4 & 1 & 1 & 2 & 3 & $(-, \otimes,-,-)$ & $\delta_{2}$ & $\mathcal{K}$ \\
\hline 390 & 446 & $2 \times 223$ & $1 \mathrm{~b}$ & $5^{2} 2^{4} 223^{4}$ & 3 & 0 & 0 & 1 & 6 & $(\times,-,-,-)$ & $\gamma$ & \\
\hline 391 & 447 & $3 \times 149$ & $1 \mathrm{~b}$ & $5^{2} 3^{4} 149^{4}$ & 4 & 1 & 1 & 3 & 4 & $(-, \otimes,-,-)$ & $\beta_{2}$ & $149 \times 5, \mathcal{K}$ \\
\hline 392 & 449 & & 2 & $449^{4}$ & 1 & 1 & 1 & 2 & 3 & $(-, \otimes,-, \times)$ & $\delta_{2}$ & $\mathcal{L}$ \\
\hline 393 & ${ }^{*} 451$ & $11 \times 41$ & 2 & $11^{4} 41^{4}$ & 1 & 2 & 3 & 6 & 3 & $(-,-, \otimes,-)$ & $\alpha_{2}$ & $\begin{array}{l}\mathcal{K}_{(11)} \mathcal{K}_{(41)}^{4}, \\
\mathfrak{K}_{(11), 1} \mathfrak{K}_{(41), 2}^{3}\end{array}$ \\
\hline 394 & 452 & $2^{2} \times 113$ & $1 \mathrm{~b}$ & $5^{2} 2^{4} 113^{4}$ & 3 & 0 & 0 & 1 & 6 & $(\times,-,-,-)$ & $\gamma$ & \\
\hline 395 & 453 & $3 \times 151$ & $1 \mathrm{~b}$ & $5^{2} 3^{4} 151^{4}$ & 4 & 1 & 1 & 3 & 4 & $(-,-,(\times),-)$ & $\beta_{2}$ & $3 \times 5^{2}, \mathcal{K}$ \\
\hline 396 & 454 & $2 \times 227$ & $1 \mathrm{~b}$ & $5^{2} 2^{4} 227^{4}$ & 3 & 0 & 0 & 1 & 6 & $(\times,-,-,-)$ & $\gamma$ & \\
\hline 397 & 455 & $5 \times 7 \times 13$ & 1a & $5^{6} 7^{4} 13^{4}$ & 16 & 0 & 0 & 1 & 6 & $(\times,-,-,--)$ & $\gamma$ & \\
\hline 398 & 456 & $2^{3} \times 3 \times 19$ & $1 \mathrm{~b}$ & $5^{2} 2^{4} 3^{4} 19^{4}$ & 13 & 1 & 2 & 5 & 6 & $(-, \times,-,-)$ & $\gamma$ & $2 \times 5,19$ \\
\hline 399 & 457 & & 2 & $457^{4}$ & 1 & 0 & 0 & 0 & 5 & $(-,-,-, \otimes)$ & $\vartheta$ & \\
\hline 400 & 458 & $2 \times 229$ & $1 \mathrm{~b}$ & $5^{2} 2^{4} 229^{4}$ & 3 & 1 & 1 & 3 & 4 & $(-, \otimes,-,-)$ & $\beta_{2}$ & $229 \times 5, \mathcal{K}$ \\
\hline
\end{tabular}


Table 15. 45 pure metacyclic fields with normalized radicands $459 \leq D \leq 508$.

\begin{tabular}{|c|c|c|c|c|c|c|c|c|c|c|c|c|}
\hline No. & $D$ & Factors & S & $P^{*}$ & $m$ & $V_{L}$ & $V_{M}$ & $V_{N}$ & $E$ & $(1,2,4,5)$ & $\mathrm{T}$ & $\mathrm{P}$ \\
\hline 401 & 459 & $3^{3} \times 17$ & $1 \mathrm{~b}$ & $5^{2} 3^{4} 17^{4}$ & 3 & 0 & 0 & 1 & 6 & $(\times,-,-,-)$ & $\gamma$ & \\
\hline 402 & 460 & $2^{2} \times 5 \times 23$ & 1a & $5^{6} 2^{4} 23^{4}$ & 16 & 0 & 0 & 1 & 6 & $(\times,-,-,-)$ & $\gamma$ & \\
\hline 403 & 461 & & $1 \mathrm{~b}$ & $5^{2} 461^{4}$ & 1 & 1 & 2 & 3 & 4 & $(-,-, \otimes,-)$ & $\delta_{1}$ & $\mathfrak{K}_{1}$ \\
\hline 404 & ${ }^{*} 462$ & $2 \times 3 \times 7 \times 11$ & $1 b$ & $5^{2} 2^{4} 3^{4} 7^{4} 11^{4}$ & 52 & 2 & 4 & 9 & 6 & $(-,-, \times,-)$ & $\gamma$ & $\begin{array}{l}5^{2} \times 7 \\
3 \times 5 \times 11^{2}\end{array}$ \\
\hline 405 & 463 & & $1 \mathrm{~b}$ & $5^{2} 463^{4}$ & 1 & 0 & 0 & 0 & 5 & $(\times,-,-,-)$ & $\varepsilon$ & \\
\hline 406 & 464 & $2^{4} \times 29$ & $1 \mathrm{~b}$ & $5^{2} 2^{4} 29^{4}$ & 3 & 1 & 1 & 3 & 4 & $(-, \otimes,-,-)$ & $\beta_{2}$ & $5, \mathcal{K}$ \\
\hline 407 & ${ }^{*} 465$ & $3 \times 5 \times 31$ & $1 \mathrm{a}$ & $5^{6} 3^{4} 31^{4}$ & 16 & 2 & 3 & $7^{*}$ & 4 & $(-,-,(\times),-)$ & $\beta_{2}$ & $5, \mathcal{K}$ \\
\hline 408 & 466 & $2 \times 233$ & $1 \mathrm{~b}$ & $5^{2} 2^{4} 233^{4}$ & 3 & 0 & 0 & 1 & 6 & $(\times,-,-,--)$ & $\gamma$ & \\
\hline 409 & 467 & & $1 \mathrm{~b}$ & $5^{2} 467^{4}$ & 1 & 0 & 0 & 0 & 5 & $(\times,-,-,-)$ & $\varepsilon$ & \\
\hline 410 & 468 & $2^{2} \times 3^{2} \times 13$ & 2 & $2^{4} 3^{4} 13^{4}$ & 3 & 0 & 0 & 1 & 6 & $(\times,-,-,-)$ & $\gamma$ & \\
\hline 411 & 469 & $7 \times 67$ & $1 \mathrm{~b}$ & $5^{2} 7^{4} 67^{4}$ & 4 & 0 & 0 & 1 & 6 & $(\times,-,-,-)$ & $\gamma$ & \\
\hline 412 & 470 & $2 \times 5 \times 47$ & 1a & $5^{6} 2^{4} 47^{4}$ & 16 & 0 & 0 & 1 & 6 & $(\times,-,-,-)$ & $\gamma$ & \\
\hline 413 & 471 & $3 \times 157$ & $1 \mathrm{~b}$ & $5^{2} 3^{4} 157^{4}$ & 4 & 0 & 0 & 1 & 6 & $(\times,-,-,--)$ & $\gamma$ & \\
\hline 414 & 472 & $2^{3} \times 59$ & $1 \mathrm{~b}$ & $5^{2} 2^{4} 59^{4}$ & 3 & 1 & 1 & 3 & 4 & $(-, \otimes,-,--)$ & $\beta_{2}$ & $2 \times 5, \mathcal{K}$ \\
\hline 415 & ${ }^{\star} 473$ & $11 \times 43$ & $1 \mathrm{~b}$ & $5^{2} 11^{4} 43^{4}$ & 4 & 2 & 3 & $7^{\star}$ & 4 & $(-,-,(\times),-)$ & $\beta_{2}$ & $11, \mathcal{L}$ \\
\hline 416 & 474 & $2 \times 3 \times 79$ & 2 & $2^{4} 3^{4} 79^{4}$ & 3 & 1 & 1 & 3 & 4 & $(-, \otimes,-,-)$ & $\beta_{2}$ & $3, \mathcal{K}$ \\
\hline 417 & 475 & $5^{2} \times 19$ & 1a & $5^{6} 19^{4}$ & 4 & 1 & 1 & 2 & 3 & $(-, \otimes,-,--)$ & $\delta_{2}$ & $\mathcal{K}$ \\
\hline 418 & 476 & $2^{2} \times 7 \times 17$ & 2 & $2^{4} 7^{4} 17^{4}$ & 4 & 0 & 0 & 1 & 6 & $(\times,-,-,-)$, & $\gamma$ & \\
\hline 419 & 477 & $3^{2} \times 53$ & $1 b$ & $5^{2} 3^{4} 53^{4}$ & 3 & 1 & 2 & 4 & 5 & $(\times,-,-,-)$ & $\varepsilon$ & $3 \times 5$ \\
\hline 420 & 478 & $2 \times 239$ & $1 \mathrm{~b}$ & $5^{2} 2^{4} 239^{4}$ & 3 & 1 & 1 & 3 & 4 & $(-, \otimes,-,-)$ & $\beta_{2}$ & $239, \mathcal{L}$ \\
\hline 421 & 479 & & $1 \mathrm{~b}$ & $5^{2} 479^{4}$ & 1 & 1 & 1 & 2 & 3 & $(-, \otimes,-,-)$ & $\delta_{2}$ & $\mathcal{L}$ \\
\hline 422 & 481 & $13 \times 37$ & $1 \mathrm{~b}$ & $5^{2} 13^{4} 37^{4}$ & 3 & 0 & 0 & 1 & 6 & $(\times,-,-,-)$ & $\gamma$ & \\
\hline 423 & ${ }^{\star} 482$ & $2 \times 241$ & 2 & $2^{4} 241^{4}$ & 1 & 1 & 2 & 4 & 5 & $(-,-, \otimes,-)$ & $\beta_{1}$ & $241, \mathfrak{K}_{2}$ \\
\hline 424 & 483 & $3 \times 7 \times 23$ & $1 \mathrm{~b}$ & $5^{2} 3^{4} 7^{4} 23^{4}$ & 12 & 1 & 2 & 5 & 6 & $(\times,-,-,-)$ & $\gamma$ & $7,3 \times 23$ \\
\hline 425 & 485 & $5 \times 97$ & $1 \mathrm{a}$ & $5^{6} 97^{4}$ & 4 & 0 & 0 & 0 & 5 & $(\times,-,-,-)$ & $\varepsilon$ & \\
\hline 426 & 487 & & $1 b$ & $5^{2} 487^{4}$ & 1 & 0 & 0 & 0 & 5 & $(\times,-,-,-)$ & $\varepsilon$ & \\
\hline 427 & 488 & $2^{3} \times 61$ & $1 \mathrm{~b}$ & $5^{2} 2^{4} 61^{4}$ & 3 & 1 & 1 & 3 & 4 & $(-,-,(\times),-)$ & $\beta_{2}$ & $2 \times 5^{2}, \mathcal{K}$ \\
\hline 428 & 489 & $3 \times 163$ & $1 b$ & $5^{2} 3^{4} 163^{4}$ & 3 & 1 & 2 & 4 & 5 & $(\times,-,-,-)$ & $\varepsilon$ & $3 \times 5^{2}$ \\
\hline 429 & 490 & $2 \times 5 \times 7^{2}$ & 1a & $5^{6} 2^{4} 7^{4}$ & 16 & 0 & 0 & 1 & 6 & $(\times,-,-,-)$ & $\gamma$ & \\
\hline 430 & 491 & & $1 b$ & $5^{2} 491^{4}$ & 1 & 1 & 1 & 2 & 3 & $(-,-, \otimes,-)$ & $\alpha_{2}$ & $\mathcal{L}, \mathfrak{K}_{1}$ \\
\hline 431 & 492 & $2^{2} \times 3 \times 41$ & $1 \mathrm{~b}$ & $5^{2} 2^{4} 3^{4} 41^{4}$ & 13 & 1 & 2 & 5 & 6 & $(-,-, \times,-)$ & $\gamma$ & $3,2 \times 5^{2}$ \\
\hline 432 & 493 & $17 \times 29$ & 2 & $17^{4} 29^{4}$ & 1 & 1 & 1 & 2 & 3 & $(-, \otimes,-,-)$ & $\delta_{2}$ & $\mathcal{K}$ \\
\hline 433 & 494 & $2 \times 13 \times 19$ & $1 b$ & $5^{2} 2^{4} 13^{4} 19^{4}$ & 13 & 1 & 2 & 5 & 6 & $(-, \times,-,-)$ & $\gamma$ & $2,13 \times 5^{2}$ \\
\hline 434 & 495 & $3^{2} \times 5 \times 11$ & 1a & $5^{6} 3^{4} 11^{4}$ & 16 & 1 & 1 & 3 & 4 & $(-,-,(\times),-)$ & $\beta_{2}$ & $11, \mathcal{L}$ \\
\hline 435 & 496 & $2^{4} \times 31$ & $1 \mathrm{~b}$ & $5^{2} 2^{4} 31^{4}$ & 3 & 1 & 1 & 3 & 4 & $(-,-,(\times),-)$ & $\beta_{2}$ & $5, \mathcal{K}$ \\
\hline
\end{tabular}




\section{Continued}

\begin{tabular}{ccccccccccccc}
\hline 436 & 497 & $7 \times 71$ & $1 \mathrm{~b}$ & $5^{2} 7^{4} 71^{4}$ & 4 & 1 & 1 & 3 & 4 & $(-,-,(\times),-)$ & $\beta_{2}$ & $7 \times 5^{3}, \mathcal{K}$ \\
437 & 498 & $2 \times 3 \times 83$ & $1 \mathrm{~b}$ & $5^{2} 2^{4} 3^{4} 83^{4}$ & 13 & 1 & 2 & 5 & 6 & $(\times,-,-,-)$ & $\gamma$ & $2^{3} \times 3,2 \times 5^{2}$ \\
438 & 499 & & 2 & $499^{4}$ & 1 & 1 & 1 & 2 & 3 & $(-, \otimes,-, \times)$ & $\delta_{2}$ & $\mathcal{L}$ \\
439 & 501 & $3 \times 167$ & 2 & $3^{4} 167^{4}$ & 1 & 0 & 0 & 0 & 5 & $(\times,-,-,-)$ & $\varepsilon$ & \\
440 & $\star 502$ & $2 \times 251$ & $1 \mathrm{~b}$ & $5^{2} 2^{4} 251^{4}$ & 4 & $2^{*}$ & $4^{*}$ & $8^{*}$ & 5 & $(-,-, \otimes,-)$ & $\beta_{1}$ & $251 \times 5, \mathfrak{K}_{2}$ \\
441 & 503 & & $1 \mathrm{~b}$ & $5^{2} 503^{4}$ & 1 & 0 & 0 & 0 & 5 & $(\times,-,-,-)$ & $\varepsilon$ & \\
442 & 504 & $2^{3} \times 3^{2} \times 7$ & $1 \mathrm{~b}$ & $5^{2} 2^{4} 3^{4} 7^{4}$ & 15 & 1 & 2 & 5 & 6 & $(\times,-,-,-)$ & $\gamma$ & $7,2 \times 5^{2}$ \\
443 & $* 505$ & $5 \times 101$ & $1 \mathrm{a}$ & $5^{6} 101^{4}$ & 4 & 1 & 1 & 3 & 4 & $(-,-,(\times), \otimes)$ & $\zeta_{2}$ & $\mathcal{K}$ \\
444 & 506 & $2 \times 11 \times 23$ & $1 \mathrm{~b}$ & $5^{2} 2^{4} 11^{4} 23^{4}$ & 13 & 1 & 2 & 5 & 6 & $(-,-, \times,-)$ & $\gamma$ & $23,2 \times 5$ \\
445 & 508 & $2^{2} \times 127$ & $1 \mathrm{~b}$ & $5^{2} 2^{4} 127^{4}$ & 3 & 0 & 0 & 1 & 6 & $(\times,-,-,-)$ & $\gamma$ & \\
\hline
\end{tabular}

Table 16. 45 pure metacyclic fields with normalized radicands $509 \leq D \leq 556$.

\begin{tabular}{|c|c|c|c|c|c|c|c|c|c|c|c|c|}
\hline No. & $D$ & Factors & S & $f^{\prime}$ & $m$ & $V_{L}$ & $V_{M}$ & $V_{N}$ & $E$ & $(1,2,4,5)$ & $\mathrm{T}$ & $\mathrm{P}$ \\
\hline 446 & 509 & & $1 b$ & $5^{2} 509^{4}$ & 1 & 1 & 1 & 2 & 3 & $(-, \otimes,-,-)$ & $\delta_{2}$ & $\mathcal{L}$ \\
\hline 447 & 510 & $2 \times 3 \times 5 \times 17$ & 1a & $5^{6} 2^{4} 3^{4} 17^{4}$ & 64 & 1 & 2 & 5 & 6 & $(\times,-,-,-)$ & $\gamma$ & $2 \times 3,2 \times 17$ \\
\hline 448 & 511 & $7 \times 73$ & $1 b$ & $5^{2} 7^{4} 73^{4}$ & 4 & 0 & 0 & 1 & 6 & $(\times,-,-,-)$ & $\gamma$ & \\
\hline 449 & 513 & $3^{3} \times 19$ & $1 b$ & $5^{2} 3^{4} 19^{4}$ & 3 & 1 & 2 & 4 & 5 & $(-, \times,-,-)$ & $\varepsilon$ & $3 \times 5^{2}$ \\
\hline 450 & 514 & $2 \times 257$ & $1 \mathrm{~b}$ & $5^{2} 2^{4} 257^{4}$ & 4 & 0 & 0 & 1 & 6 & $(\times,-,-,-)$ & $\gamma$ & \\
\hline 451 & 515 & $5 \times 103$ & 1a & $5^{6} 103^{4}$ & 4 & 0 & 0 & 0 & 5 & $(\times,-,-,--)$ & $\varepsilon$ & \\
\hline 452 & 516 & $2^{2} \times 3 \times 43$ & $1 \mathrm{~b}$ & $5^{2} 2^{4} 3^{4} 43^{4}$ & 12 & 1 & 2 & 5 & 6 & $(\times,-,-,-)$ & $\gamma$ & $2 \times 3^{2}, 2 \times 5^{4}$ \\
\hline 453 & $\star 517$ & $11 \times 47$ & $1 \mathrm{~b}$ & $5^{2} 11^{4} 47^{4}$ & 3 & 2 & 3 & 6 & 3 & $(-,-, \otimes,-)$ & $\beta_{1}$ & $11 \times 5^{2}, \mathfrak{K}_{2}$ \\
\hline 454 & 518 & $2 \times 7 \times 37$ & 2 & $2^{4} 7^{4} 37^{4}$ & 4 & 0 & 0 & 1 & 6 & $(\times,-,-,-)$ & $\gamma$ & \\
\hline 455 & 519 & $3 \times 173$ & $1 \mathrm{~b}$ & $5^{2} 3^{4} 173^{4}$ & 3 & 0 & 0 & 1 & 6 & $(\times,-,-,--)$ & $\gamma$ & \\
\hline 456 & 520 & $2^{3} \times 5 \times 13$ & $1 \mathrm{a}$ & $5^{6} 2^{4} 13^{4}$ & 16 & 1 & 2 & 4 & 5 & $(\times,-,-,-)$, & $\varepsilon$ & 2 \\
\hline 457 & 521 & & $1 \mathrm{~b}$ & $5^{2} 521^{4}$ & 1 & 1 & 2 & 3 & 4 & $(-,-, \otimes,-)$ & $\delta_{1}$ & $\mathfrak{K}_{2}$ \\
\hline 458 & 522 & $2 \cdot \times 3^{2} \times 29$ & $1 \mathrm{~b}$ & $5^{2} 2^{4} 3^{4} 29^{4}$ & 13 & 1 & 2 & 5 & 6 & $(-, \times,-,-)$ & $\gamma$ & $2,29 \times 5$ \\
\hline 459 & 523 & & $1 b$ & $5^{2} 523^{4}$ & 1 & 0 & 0 & 0 & 5 & $(\times,-,-,-)$ & $\varepsilon$ & \\
\hline 460 & 524 & $2^{2} \times 131$ & 2 & $2^{4} 131^{4}$ & 1 & 1 & 1 & 2 & 3 & $(-,-, \otimes,-)$ & $\alpha_{2}$ & $\mathcal{K}, \mathfrak{K}_{1}$ \\
\hline 461 & 525 & $3 \times 5^{2} \times 7$ & 1a & $5^{6} 3^{4} 7^{4}$ & 16 & 0 & 0 & 1 & 6 & $(\times,-,-,-)$ & $\gamma$ & \\
\hline 462 & 526 & $2 \times 263$ & 2 & $2^{4} 263^{4}$ & 1 & 0 & 0 & 0 & 5 & $(\times,-,-,--)$ & $\varepsilon$ & \\
\hline 463 & 527 & $17 \times 31$ & $1 b$ & $5^{2} 17^{4} 31^{4}$ & 3 & 1 & 1 & 3 & 4 & $(-,-,(\times),-)$ & $\beta_{2}$ & $5, \mathcal{K}$ \\
\hline 464 & 528 & $2^{4} \times 3 \times 11$ & $1 b$ & $5^{2} 2^{4} 3^{4} 11^{4}$ & 13 & 1 & 2 & 5 & 6 & $(-,-, \times,-)$ & $\gamma$ & $2 \times 5,3 \times 5^{3}$ \\
\hline 465 & 530 & $2 \times 5 \times 53$ & 1a & $5^{6} 2^{4} 53^{4}$ & 16 & 0 & 0 & 1 & 6 & $(\times,-,-,-)$ & $\gamma$ & \\
\hline 466 & 531 & $3^{2} \times 59$ & $1 b$ & $5^{2} 3^{4} 59^{4}$ & 3 & 1 & 1 & 3 & 4 & $(-, \otimes,-,-)$ & $\beta_{2}$ & $3, \mathcal{L}$ \\
\hline 467 & ${ }^{*} 532$ & $2^{2} \times 7 \times 19$ & 2 & $2^{4} 7^{4} 19^{4}$ & 4 & 1 & 2 & 4 & 5 & $(-, \times,-,-)$ & $\varepsilon$ & $2 \times 7$ \\
\hline 468 & 533 & $13 \times 41$ & $1 b$ & $5^{2} 13^{4} 41^{4}$ & 3 & 1 & 1 & 3 & 4 & $(-,-,(\times),-)$ & $\beta_{2}$ & $13 \times 5^{2}, \mathcal{K}$ \\
\hline 469 & 534 & $2 \times 3 \times 89$ & $1 b$ & $5^{2} 2^{4} 3^{4} 89^{4}$ & 13 & 1 & 2 & 5 & 6 & $(-, \times,-,-)$ & $\gamma$ & $3,89 \times 5$ \\
\hline
\end{tabular}




\section{Continued}

\begin{tabular}{|c|c|c|c|c|c|c|c|c|c|c|c|c|}
\hline 470 & 535 & $5 \times 107$ & la & $5^{6} 107^{4}$ & 4 & 0 & 0 & 1 & 6 & $(-,-,-, \otimes)$ & $\eta$ & \\
\hline 471 & 536 & $2^{3} \times 67$ & $1 b$ & $5^{2} 2^{4} 67^{4}$ & 3 & 0 & 0 & 1 & 6 & $(\times,-,-,-)$ & $\gamma$ & \\
\hline 472 & 537 & $3 \times 179$ & $1 b$ & $5^{2} 3^{4} 179^{4}$ & 3 & 1 & 1 & 3 & 4 & $(-, \otimes,-,-)$ & $\beta_{2}$ & $3 \times 5^{2}, \mathcal{K}$ \\
\hline 473 & 538 & $2 \times 269$ & $1 b$ & $5^{2} 2^{4} 269^{4}$ & 3 & 1 & 1 & 3 & 4 & $(-, \otimes,-,-)$ & $\beta_{2}$ & $2, \mathcal{L}$ \\
\hline 474 & 539 & $7^{2} \times 11$ & $1 b$ & $5^{2} 7^{4} 11^{4}$ & 4 & 1 & 1 & 3 & 4 & $(-,-,(\times),-)$ & $\beta_{2}$ & $11 \times 5, \mathcal{K}$ \\
\hline 475 & 540 & $2^{2} \times 3^{3} \times 5$ & 1a & $5^{6} 2^{4} 3^{4}$ & 16 & 0 & 0 & 1 & 6 & $(\times,-,-,-)$ & $\gamma$ & \\
\hline 476 & 541 & & $1 \mathrm{~b}$ & $5^{2} 541^{4}$ & 1 & 2 & 2 & 4 & 1 & $(-,-, \otimes,-)$ & $\alpha_{2}$ & $\mathcal{K}, \mathfrak{K}_{1}$ \\
\hline 477 & 542 & $2 \times 271$ & $1 b$ & $5^{2} 2^{4} 271^{4}$ & 3 & 1 & 1 & 3 & 4 & $(-,-,(\times),-)$ & $\beta_{2}$ & $5, \mathcal{K}$ \\
\hline 478 & 543 & $3 \times 181$ & 2 & $3^{4} 181^{4}$ & 1 & 1 & 1 & 2 & 3 & $(-,-, \otimes,-)$ & $\alpha_{2}$ & $\mathcal{K}, \mathfrak{K}_{1}$ \\
\hline 479 & 545 & $5 \times 109$ & 1a & $5^{6} 109^{4}$ & 4 & 1 & 1 & 2 & 3 & $(-, \otimes,-,-)$ & $\delta_{2}$ & $\mathcal{K}$ \\
\hline 480 & *546 & $2 \times 3 \times 7 \times 13$ & $1 b$ & $5^{2} 2^{4} 3^{4} 7^{4} 13^{4}$ & 52 & 2 & 4 & 9 & 6 & $(\times,-,-,-)$ & $\gamma$ & $\begin{array}{l}3 \times 5 \times 13, \\
5^{2} \times 7 \times 13\end{array}$ \\
\hline 481 & 547 & & $1 b$ & $5^{2} 547^{4}$ & 1 & 0 & 0 & 0 & 5 & $(\times,-,-,-)$ & $\varepsilon$ & \\
\hline 482 & 548 & $2^{2} \times 137$ & $1 \mathrm{~b}$ & $5^{2} 2^{4} 137^{4}$ & 3 & 0 & 0 & 1 & 6 & $(\times,-,-,-)$ & $\gamma$ & \\
\hline 483 & 549 & $3^{2} \times 61$ & 2 & $3^{4} 61^{4}$ & 3 & 2 & 2 & 4 & 1 & $(-,-, \otimes,-)$ & $\alpha_{2}$ & $\mathcal{K}, \mathfrak{K}_{1}$ \\
\hline 484 & $\star$ *550 & $2 \times 5^{2} \times 11$ & $1 \mathrm{a}$ & $5^{6} 2^{4} 11^{4}$ & 16 & 2 & 3 & 6 & 3 & $(-,-, \otimes,-)$ & $\alpha_{2}$ & $\mathcal{K}, \mathfrak{K}_{1}$ \\
\hline 485 & $\star 551$ & $19 \times 29$ & 2 & $19^{4} 29^{4}$ & 1 & 2 & 2 & 5 & 2 & $(-, \otimes,-,-)$ & $\alpha_{3}$ & $\mathcal{K}_{(19)}, \mathcal{K}_{(29)}$ \\
\hline 486 & 552 & $2^{3} \times 3 \times 23$ & $1 b$ & $5^{2} 2^{4} 3^{4} 23^{4}$ & 13 & 1 & 2 & 5 & 6 & $(\times,-,-,-)$ & $\gamma$ & $23,3 \times 5$ \\
\hline 487 & 553 & $7 \times 79$ & $1 \mathrm{~b}$ & $5^{2} 7^{4} 79^{4}$ & 4 & 1 & 2 & 4 & 5 & $(-, \times,-,-)$ & $\varepsilon$ & 5 \\
\hline 488 & 554 & $2 \times 277$ & $1 b$ & $5^{2} 2^{4} 277^{4}$ & 3 & 0 & 0 & 1 & 6 & $(\times,-,-,-)$ & $\gamma$ & \\
\hline 489 & 555 & $3 \times 5 \times 37$ & 1a & $5^{6} 3^{4} 37^{4}$ & 16 & 0 & 0 & 1 & 6 & $(\times,-,-,-)$ & $\gamma$ & \\
\hline 490 & 556 & $2^{2} \times 139$ & $1 b$ & $5^{2} 2^{4} 139^{4}$ & 3 & 1 & 1 & 3 & 4 & $(-, \otimes,-,-)$ & $\beta_{2}$ & $2 \times 5^{2}, \mathcal{K}$ \\
\hline
\end{tabular}

Table 17. 45 pure metacyclic fields with normalized radicands $557 \leq D \leq 604$.

\begin{tabular}{ccccccccccccc}
\hline No. & $D$ & Factors & $\mathrm{S}$ & $f^{*}$ & $m$ & $V_{L}$ & $V_{M}$ & $V_{N} E$ & $(1,2,4,5)$ & $\mathrm{T}$ & $\mathrm{P}$ \\
\hline 491 & 557 & & 2 & $557^{4}$ & 1 & 0 & 0 & 0 & 5 & $(-,-,-, \otimes)$ & $\vartheta$ & \\
492 & 558 & $2 \times 3^{2} \times 31$ & $1 \mathrm{~b}$ & $5^{2} 2^{4} 3^{4} 31^{4}$ & 13 & 1 & 2 & 5 & 6 & $(-,-, \times,-)$ & $\gamma$ & $5,3 \times 31$ \\
493 & 559 & $13 \times 43$ & $1 \mathrm{~b}$ & $5^{2} 13^{4} 43^{4}$ & 4 & 0 & 0 & 1 & 6 & $(\times,-,-,-)$ & $\gamma$ & \\
494 & 560 & $2^{4} \times 5 \times 7$ & $1 \mathrm{a}$ & $5^{6} 2^{4} 7^{4}$ & 16 & 0 & 0 & 1 & 6 & $(\times,-,-,-)$ & $\gamma$ & \\
495 & 561 & $3 \times 11 \times 17$ & $1 \mathrm{~b}$ & $5^{2} 3^{4} 11^{4} 17^{4}$ & 13 & 1 & 2 & 5 & 6 & $(-,-, \times,-)$ & $\gamma$ & $11 \times 5^{3}, 17 \times 5^{4}$ \\
496 & 562 & $2 \times 281$ & $1 \mathrm{~b}$ & $5^{2} 2^{4} 281^{4}$ & 3 & 1 & 2 & 4 & 5 & $(-,-, \times,-)$ & $\varepsilon$ & \\
497 & 563 & & $1 \mathrm{~b}$ & $5^{2} 563^{4}$ & 1 & 0 & 0 & 0 & 5 & $(\times,-,-,-)$ & $\varepsilon$ & \\
498 & 564 & $2^{2} \times 3 \times 47$ & $1 \mathrm{~b}$ & $5^{2} 2^{4} 3^{4} 47^{4}$ & 13 & 1 & 2 & 5 & 6 & $(\times,-,-,-)$ & $\gamma$ & $2 \times 47,2 \times 5^{2}$ \\
499 & 565 & $5 \times 113$ & $1 \mathrm{a}$ & $5^{6} 113^{4}$ & 4 & 0 & 0 & 0 & 5 & $(\times,-,-,-)$ & $\varepsilon$ & \\
500 & 566 & $2 \times 283$ & $1 \mathrm{~b}$ & $5^{2} 2^{4} 283^{4}$ & 3 & 0 & 0 & 1 & 6 & $(\times,-,-,-)$ & $\gamma$ & \\
\hline
\end{tabular}




\section{Continued}

\begin{tabular}{|c|c|c|c|c|c|c|c|c|c|c|c|c|}
\hline 501 & 567 & $3^{4} \times 7$ & $1 \mathrm{~b}$ & $5^{2} 3^{4} 7^{4}$ & 4 & 0 & 0 & 1 & 6 & $(\times,-,-,-)$ & $\gamma$ & \\
\hline 502 & 568 & $2^{3} \times 71$ & 2 & $2^{4} 71^{4}$ & 1 & 1 & 1 & 2 & 3 & $(-,-, \otimes,-)$ & $\alpha_{2}$ & $\mathcal{K}, \mathfrak{K}_{1}$ \\
\hline 503 & 569 & & $1 \mathrm{~b}$ & $5^{2} 569^{4}$ & 1 & 1 & 1 & 2 & 3 & $(-, \otimes,-,-)$ & $\delta_{2}$ & $\mathcal{L}$ \\
\hline 504 & $\star 570$ & $2 \times 3 \times 5 \times 19$ & 1a & $5^{6} 2^{4} 3^{4} 19^{4}$ & 64 & 1 & 2 & 5 & 6 & $(-, \times,-,-)$ & $\gamma$ & $2^{4} \times 3,2^{4} \times 5$ \\
\hline 505 & 571 & & $1 \mathrm{~b}$ & $5^{2} 571^{4}$ & 1 & 2 & 2 & 4 & 1 & $(-,-, \otimes,-)$ & $\alpha_{2}$ & $\mathcal{L}, \mathfrak{K}_{1}$ \\
\hline 506 & 572 & $2^{2} \times 11 \times 13$ & $1 b$ & $5^{2} 2^{4} 11^{4} 13^{4}$ & 13 & 2 & 3 & 6 & 3 & $(-,-, \otimes,-)$ & $\beta_{1}$ & $2 \times 13^{2} \times 5^{3}, \mathfrak{K}_{2}$ \\
\hline 507 & 573 & $3 \times 191$ & $1 \mathrm{~b}$ & $5^{2} 3^{4} 191^{4}$ & 3 & 1 & 1 & 3 & 4 & $(-,-,(\times),-)$ & $\beta_{2}$ & $5, \mathcal{K}$ \\
\hline 508 & *574 & $2 \times 7 \times 41$ & 2 & $2^{4} 7^{4} 41^{4}$ & 4 & 1 & 1 & 3 & 4 & $(-,-,(\times),-)$ & $\beta_{2}$ & $41, \mathcal{L}$ \\
\hline 509 & 575 & $5^{2} \times 23$ & 1a & $5^{6} 23^{4}$ & 4 & 0 & 0 & 0 & 5 & $(\times,-,-,--)$ & $\varepsilon$ & \\
\hline 510 & 577 & & $1 \mathrm{~b}$ & $5^{2} 577^{4}$ & 1 & 0 & 0 & 0 & 5 & $(\times,-,-,--)$ & $\varepsilon$ & \\
\hline 511 & 579 & $3 \times 193$ & $1 \mathrm{~b}$ & $5^{2} 3^{4} 193^{4}$ & 4 & 0 & 0 & 1 & 6 & $(\times,-,-,--)$ & $\gamma$ & \\
\hline 512 & 580 & $2^{2} \times 5 \times 29$ & 1a & $5^{6} 2^{4} 29^{4}$ & 16 & 1 & 1 & 3 & 4 & $(-, \otimes,-,-)$ & $\beta_{2}$ & $2^{4} \times 5, \mathcal{K}$ \\
\hline 513 & 581 & $7 \times 83$ & $1 \mathrm{~b}$ & $5^{2} 7^{4} 83^{4}$ & 4 & 0 & 0 & 1 & 6 & $(\times,-,-,-)$ & $\gamma$ & \\
\hline 514 & 582 & $2 \times 3 \times 97$ & 2 & $2^{4} 3^{4} 97^{4}$ & 3 & 0 & 0 & 1 & 6 & $(\times,-,-,-)$ & $\gamma$ & \\
\hline 515 & 583 & $11 \times 53$ & $1 \mathrm{~b}$ & $5^{2} 11^{4} 53^{4}$ & 3 & 1 & 1 & 3 & 4 & $(-,-,(\times),-)$ & $\beta_{2}$ & $53 \times 5, \mathcal{K}$ \\
\hline 516 & 584 & $2^{3} \times 73$ & $1 \mathrm{~b}$ & $5^{2} 2^{4} 73^{4}$ & 3 & 0 & 0 & 1 & 6 & $(\times,-,-,--)$ & $\gamma$ & \\
\hline 517 & 585 & $3^{2} \times 5 \times 13$ & 1a & $5^{6} 3^{4} 13^{4}$ & 16 & 0 & 0 & 1 & 6 & $(\times,-,-,-)$ & $\gamma$ & \\
\hline 518 & 586 & $2 \times 293$ & $1 \mathrm{~b}$ & $5^{2} 2^{4} 293^{4}$ & 4 & 1 & 2 & 4 & 5 & $(\times,-,-,-)$ & $\varepsilon$ & 2 \\
\hline 519 & 587 & & $1 \mathrm{~b}$ & $5^{2} 587^{4}$ & 1 & 0 & 0 & 0 & 5 & $(\times,-,-,--)$ & $\varepsilon$ & \\
\hline 520 & 589 & $19 \times 31$ & $1 \mathrm{~b}$ & $5^{2} 19^{4} 31^{4}$ & 3 & 2 & 2 & 5 & 2 & $(-, \otimes,(\times),-)$ & $\alpha_{3}$ & $\mathcal{K}_{(19)}, \mathcal{K}_{(31)}$ \\
\hline 521 & $\star 590$ & $2 \times 5 \times 59$ & $1 \mathrm{a}$ & $5^{6} 2^{4} 59^{4}$ & 16 & 2 & 3 & $7^{*}$ & 4 & $(-, \otimes,-,-)$ & $\beta_{2}$ & $5, \mathcal{K}$ \\
\hline 522 & 591 & $3 \times 197$ & $1 \mathrm{~b}$ & $5^{2} 3^{4} 197^{4}$ & 3 & 0 & 0 & 1 & 6 & $(\times,-,-,--)$ & $\gamma$ & \\
\hline 523 & 592 & $2^{4} \times 37$ & $1 \mathrm{~b}$ & $5^{2} 2^{4} 37^{4}$ & 3 & 0 & 0 & 1 & 6 & $(\times,-,-,--)$ & $\gamma$ & \\
\hline 524 & 593 & & 2 & $593^{4}$ & 1 & 0 & 0 & 0 & 5 & $(-,-,-, \otimes)$ & $\vartheta$ & \\
\hline 525 & 594 & $2 \times 3^{3} \times 11$ & $1 \mathrm{~b}$ & $5^{2} 2^{4} 3^{4} 11^{4}$ & 13 & 1 & 2 & 5 & 6 & $(-,-, \times,-)$ & $\gamma$ & $11,3^{2} \times 5$ \\
\hline 526 & 595 & $5 \times 7 \times 17$ & 1a & $5^{6} 7^{4} 17^{4}$ & 16 & 0 & 0 & 1 & 6 & $(\times,-,-,-)$ & $\gamma$ & \\
\hline 527 & 596 & $2^{2} \times 149$ & $1 \mathrm{~b}$ & $5^{2} 2^{4} 149^{4}$ & 4 & 1 & 2 & 4 & 5 & $(-, \times,-,-)$ & $\varepsilon$ & $2 \times 5^{2}$ \\
\hline 528 & 597 & $3 \times 199$ & $1 \mathrm{~b}$ & $5^{2} 3^{4} 199^{4}$ & 4 & 1 & 1 & 3 & 4 & $(-, \otimes,-,-)$ & $\beta_{2}$ & $3^{4} \times 5, \mathcal{K}$ \\
\hline 529 & 598 & $2 \times 13 \times 23$ & $1 b$ & $5^{2} 2^{4} 13^{4} 23^{4}$ & 13 & 1 & 2 & 5 & 6 & $(\times,-,-,-)$ & $\gamma$ & $2^{4} \times 5,13 \times 5^{3}$ \\
\hline 530 & 599 & & 2 & $599^{4}$ & 1 & 1 & 1 & 2 & 3 & $(-, \otimes,-, \times)$ & $\delta_{2}$ & $\mathcal{L}$ \\
\hline 531 & 600 & $2^{3} \times 3 \times 5^{2}$ & 1a & $5^{6} 2^{4} 3^{4}$ & 16 & 0 & 0 & 1 & 6 & $(\times,-,-,-)$ & $\gamma$ & \\
\hline 532 & 601 & & 2 & $601^{4}$ & 1 & 1 & 1 & 2 & 3 & $(-,-, \otimes, \times)$ & $\alpha_{2}$ & $\mathcal{L}, \mathfrak{K}_{2}$ \\
\hline 533 & ${ }^{*} 062$ & $2 \times 7 \times 43$ & $1 \mathrm{~b}$ & $5^{2} 2^{4} 7^{4} 43^{4}$ & 16 & 1 & 2 & 5 & 6 & $(\times,-,-,-)$ & $\gamma$ & 2,7 \\
\hline 534 & 603 & $3^{2} \times 67$ & $1 \mathrm{~b}$ & $5^{2} 3^{4} 67^{4}$ & 3 & 0 & 0 & 1 & 6 & $(\times,-,-,-)$ & $\gamma$ & \\
\hline 535 & 604 & $2^{2} \times 151$ & $1 \mathrm{~b}$ & $5^{2} 2^{4} 151^{4}$ & 4 & 1 & 2 & 4 & 5 & $(-,-, \otimes,-)$ & $\beta_{1}$ & $2, \mathfrak{K}_{1}$ \\
\hline
\end{tabular}


Table 18. 45 pure metacyclic fields with normalized radicands $605 \leq D \leq 653$.

\begin{tabular}{|c|c|c|c|c|c|c|c|c|c|c|c|c|}
\hline No. & $D$ & Factors & $S$ & $f^{*}$ & $m$ & $V_{L}$ & $V_{M}$ & $V_{N}$ & $E$ & $(1,2,4,5)$ & $\mathrm{T}$ & $\mathrm{P}$ \\
\hline 536 & 605 & $5 \times 11^{2}$ & $1 \mathrm{a}$ & $5^{6} 11^{4}$ & 4 & 1 & 1 & 2 & 3 & $(-,-, \otimes,-)$ & $\alpha_{2}$ & $\mathcal{K}, \mathfrak{K}_{1}$ \\
\hline 537 & ${ }^{*} 606$ & $2 \times 3 \times 101$ & $1 b$ & $5^{2} 2^{4} 3^{4} 101^{4}$ & 12 & 1 & 2 & 5 & 6 & $(-,-, \times,-)$ & $\gamma$ & $2 \times 5,101$ \\
\hline 538 & 607 & & 2 & $607^{4}$ & 1 & 0 & 0 & 0 & 5 & $(-,-,-, \otimes)$ & $\vartheta$ & \\
\hline 539 & ${ }^{*} 609$ & $3 \times 7 \times 29$ & $1 \mathrm{~b}$ & $5^{2} 3^{4} 7^{4} 29^{4}$ & 12 & 2 & 3 & 7 & 4 & $(-, \otimes,-,-)$ & $\beta_{2}$ & $7^{2} \times 5, \mathcal{K}$ \\
\hline 540 & 610 & $2 \times 5 \times 61$ & 1a & $5^{6} 2^{4} 61^{4}$ & 16 & 1 & 1 & 3 & 4 & $(-,-,(\times),-)$ & $\beta_{2}$ & $2 \times 5^{2}, \mathcal{K}$ \\
\hline 541 & 611 & $13 \times 47$ & $1 \mathrm{~b}$ & $5^{2} 13^{4} 47^{4}$ & 3 & 0 & 0 & 1 & 6 & $(\times,-,-,--)$ & $\gamma$ & \\
\hline 542 & 612 & $2^{2} \times 3^{2} \times 17$ & $1 \mathrm{~b}$ & $5^{2} 2^{4} 3^{4} 17^{4}$ & 13 & 1 & 2 & 5 & 6 & $(\times,-,-,--)$ & $\gamma$ & $17,3^{2} \times 5$ \\
\hline 543 & 613 & & $1 b$ & $5^{2} 613^{4}$ & 1 & 0 & 0 & 0 & 5 & $(\times,-,-,--)$ & $\varepsilon$ & \\
\hline 544 & 614 & $2 \times 307$ & $1 b$ & $5^{2} 2^{4} 307^{4}$ & 4 & 1 & 2 & 4 & 5 & $(\times,-,-,-)$ & $\varepsilon$ & 2 \\
\hline 545 & 615 & $3 \times 5 \times 41$ & $1 \mathrm{a}$ & $5^{6} 3^{4} 41^{4}$ & 16 & 1 & 1 & 3 & 4 & $(-,-,(\times),-)$ & $\beta_{2}$ & $3, \mathcal{K}$ \\
\hline 546 & 616 & $2^{3} \times 7 \times 11$ & $1 \mathrm{~b}$ & $5^{2} 2^{4} 7^{4} 11^{4}$ & 12 & 2 & 3 & 7 & 4 & $(-,-,(\times),-)$ & $\beta_{2}$ & $7 \times 5^{2}, \mathcal{K}$ \\
\hline 547 & 617 & & $1 \mathrm{~b}$ & $5^{2} 617^{4}$ & 1 & 0 & 0 & 0 & 5 & $(\times,-,-,-)$ & $\varepsilon$ & \\
\hline 548 & 618 & $2 \times 3 \times 103$ & 2 & $2^{4} 3^{4} 103^{4}$ & 3 & 0 & 0 & 1 & 6 & $(\times,-,-,--)$ & $\gamma$ & \\
\hline 549 & 619 & & $1 \mathrm{~b}$ & $5^{2} 619^{4}$ & 1 & 1 & 1 & 2 & 3 & $(-, \otimes,-,-)$ & $\delta_{2}$ & $\mathcal{L}$ \\
\hline 550 & ${ }^{*} 620$ & $2^{2} \times 5 \times 31$ & $1 \mathrm{a}$ & $5^{6} 2^{4} 31^{4}$ & 16 & 2 & $4^{*}$ & $8^{*}$ & 5 & $(-,-, \otimes,-)$ & $\beta_{1}$ & $5, \mathfrak{K}_{1}$ \\
\hline 551 & 621 & $3^{3} \times 23$ & $1 b$ & $5^{2} 3^{4} 23^{4}$ & 3 & 0 & 0 & 1 & 6 & $(\times,-,-,-)$ & $\gamma$ & \\
\hline 552 & 622 & $2 \times 311$ & $1 \mathrm{~b}$ & $5^{2} 2^{4} 311^{4}$ & 3 & 2 & 2 & 4 & 1 & $(-,-, \otimes,-)$ & $\alpha_{2}$ & $\mathcal{K}, \mathfrak{K}_{1}$ \\
\hline 553 & 623 & $7 \times 89$ & $1 \mathrm{~b}$ & $5^{2} 7^{4} 89^{4}$ & 4 & 1 & 1 & 3 & 4 & $(-, \otimes,-,-)$ & $\beta_{2}$ & $7 \times 5, \mathcal{K}$ \\
\hline 554 & 624 & $2^{4} \times 3 \times 13$ & 2 & $2^{4} 3^{4} 13^{4}$ & 3 & 0 & 0 & 1 & 6 & $(\times,-,-,-)$ & $\gamma$ & \\
\hline 555 & 626 & $2 \times 313$ & 2 & $2^{4} 313^{4}$ & 1 & 0 & 0 & 0 & 5 & $(\times,-,-,-)$ & $\varepsilon$ & \\
\hline 556 & ${ }^{*} 627$ & $3 \times 11 \times 19$ & $1 b$ & $5^{2} 3^{4} 11^{4} 19^{4}$ & 13 & 3 & 4 & 9 & 2 & $(-, \otimes,(\times),-)$ & $\alpha_{3}$ & $\mathcal{K}_{(11)}, \mathcal{K}_{(19)}$ \\
\hline 557 & 628 & $2^{2} \times 157$ & $1 \mathrm{~b}$ & $5^{2} 2^{4} 157^{4}$ & 4 & 0 & 0 & 1 & 6 & $(\times,-,-,-)$ & $\gamma$ & \\
\hline 558 & 629 & $17 \times 37$ & $1 \mathrm{~b}$ & $5^{2} 17^{4} 37^{4}$ & 3 & 0 & 0 & 1 & 6 & $(\times,-,-,--)$ & $\gamma$ & \\
\hline 559 & 630 & $2 \times 3^{2} \times 5 \times 7$ & la & $5^{6} 2^{4} 3^{4} 7^{4}$ & 64 & 1 & 2 & 5 & 6 & $(\times,-,-,-)$ & $\gamma$ & 3,5 \\
\hline 560 & 631 & & $1 \mathrm{~b}$ & $5^{2} 631^{4}$ & 1 & 1 & 1 & 2 & 3 & $(-,-, \otimes,-)$ & $\alpha_{2}$ & $\mathcal{L}, \mathfrak{K}_{1}$ \\
\hline 561 & 632 & $2^{3} \times 79$ & 2 & $2^{4} 79^{4}$ & 1 & 1 & 1 & 2 & 3 & $(-, \otimes,-,-)$ & $\delta_{2}$ & $\mathcal{K}$ \\
\hline 562 & 633 & $3 \times 211$ & $1 \mathrm{~b}$ & $5^{2} 3^{4} 211^{4}$ & 3 & 1 & 2 & 4 & 5 & $(-,-, \times,-)$ & $\varepsilon$ & $3 \times 5$ \\
\hline 563 & 634 & $2 \times 317$ & $1 \mathrm{~b}$ & $5^{2} 2^{4} 317^{4}$ & 3 & 0 & 0 & 1 & 6 & $(\times,-,-,-)$ & $\gamma$ & \\
\hline 564 & 635 & $5 \times 127$ & la & $5^{6} 127^{4}$ & 4 & 0 & 0 & 0 & 5 & $(\times,-,-,-)$ & $\varepsilon$ & \\
\hline 565 & 636 & $2^{2} \times 3 \times 53$ & $1 b$ & $5^{2} 2^{4} 3^{4} 53^{4}$ & 13 & 1 & 2 & 5 & 6 & $(\times,-,-,-)$ & $\gamma$ & $3^{2} \times 5,53$ \\
\hline 566 & 637 & $7^{2} \times 13$ & $1 \mathrm{~b}$ & $5^{2} 7^{4} 13^{4}$ & 4 & 0 & 0 & 1 & 6 & $(\times,-,-,-)$ & $\gamma$ & \\
\hline 567 & ${ }^{*} 638$ & $2 \times 11 \times 29$ & $1 \mathrm{~b}$ & $5^{2} 2^{4} 11^{4} 29^{4}$ & 13 & 2 & 3 & 7 & 4 & $(-, \otimes,(\times),-)$ & $\beta_{2}$ & $\begin{array}{l}2^{4} \times 11 \times 5 \\
\mathcal{K}_{(11)} \times \mathcal{K}_{(29)}^{4}\end{array}$ \\
\hline 568 & 639 & $3^{2} \times 71$ & $1 \mathrm{~b}$ & $5^{2} 3^{4} 71^{4}$ & 3 & 1 & 1 & 3 & 4 & $(-,-,(\times),-)$ & $\beta_{2}$ & $3^{2} \times 5, \mathcal{K}$ \\
\hline 569 & 641 & & $1 b$ & $5^{2} 641^{4}$ & 1 & 1 & 2 & 4 & 5 & $(-,-, \otimes,-)$ & $\beta_{1}$ & $5, \mathfrak{K}_{2}$ \\
\hline 570 & 642 & $2 \times 3 \times 107$ & $1 \mathrm{~b}$ & $5^{2} 2^{4} 3^{4} 107^{4}$ & 12 & 1 & 2 & 5 & 6 & $(\times,-,-,-)$ & $\gamma$ & $107,3 \times 5$ \\
\hline
\end{tabular}




\section{Continued}

\begin{tabular}{ccccccccccccc}
\hline 571 & 643 & & 2 & $643^{4}$ & 1 & 0 & 0 & 0 & 5 & $(-,-,-, \otimes)$ & $\vartheta$ & \\
572 & 644 & $2^{2} \times 7 \times 23$ & $1 \mathrm{~b}$ & $5^{2} 2^{4} 7^{4} 23^{4}$ & 12 & 1 & 2 & 5 & 6 & $(\times,-,-,-)$ & $\gamma$ & $2 \times 5,7 \times 5$ \\
573 & 645 & $3 \times 5 \times 43$ & $1 \mathrm{a}$ & $5^{6} 3^{4} 43^{4}$ & 16 & 1 & 2 & 4 & 5 & $(\times,-,-,-)$ & $\varepsilon$ & 43 \\
574 & 646 & $2 \times 17 \times 19$ & $1 \mathrm{~b}$ & $5^{2} 2^{4} 17^{4} 19^{4}$ & 13 & 1 & 2 & 5 & 6 & $(-, \times,-,-)$ & $\gamma$ & $2 \times 5,17$ \\
575 & 647 & & $1 \mathrm{~b}$ & $5^{2} 647^{4}$ & 1 & 0 & 0 & 0 & 5 & $(\times,-,-,-)$ & $\varepsilon$ & \\
576 & $* 649$ & $11 \times 59$ & 2 & $11^{4} 59^{4}$ & 1 & 2 & 2 & 5 & 2 & $(-, \otimes,(\times),-)$ & $\alpha_{3}$ & $\mathcal{K}_{(11)}, \mathcal{K}_{(59)}$ \\
577 & 650 & $2 \times 5^{2} \times 13$ & $1 \mathrm{a}$ & $5^{6} 2^{4} 13^{4}$ & 16 & 0 & 0 & 1 & 6 & $(\times,-,-,-)$ & $\gamma$ & \\
578 & 651 & $3 \times 7 \times 31$ & 2 & $3^{4} 7^{4} 31^{4}$ & 4 & 1 & 1 & 3 & 4 & $(-,-,(\times),-)$ & $\beta_{2}$ & $3^{2} \times 7, \mathcal{K}$ \\
579 & 652 & $2^{2} \times 163$ & $1 \mathrm{~b}$ & $5^{2} 2^{4} 163^{4}$ & 3 & 1 & 2 & 4 & 5 & $(\times,-,-,-)$ & $\varepsilon$ & 5 \\
580 & 653 & & $1 \mathrm{~b}$ & $5^{2} 653^{4}$ & 1 & 0 & 0 & 0 & 5 & $(\times,-,-,-)$ & $\varepsilon$ & \\
\hline
\end{tabular}

Table 19. 44 pure metacyclic fields with normalized radicands $654 \leq D \leq 701$.

\begin{tabular}{|c|c|c|c|c|c|c|c|c|c|c|c|c|}
\hline No. & $D$ & Factors & $S$ & $f^{*}$ & $m$ & $V_{L}$ & $V_{M}$ & $V_{N}$ & $E$ & $(1,2,4,5)$ & $\mathrm{T}$ & $\mathrm{P}$ \\
\hline 581 & 654 & $2 \times 3 \times 109$ & $1 \mathrm{~b}$ & $5^{2} 2^{4} 3^{4} 109^{4}$ & 13 & 1 & 2 & 5 & 6 & $(\times,-,-,-)$ & $\gamma$ & $3 \times 5,109$ \\
\hline 582 & 655 & $5 \times 131$ & 1a & $5^{6} 131^{4}$ & 4 & 1 & 1 & 2 & 3 & $(-,-, \otimes,-)$ & $\alpha_{2}$ & $\mathcal{K}, \mathfrak{K}_{1}$ \\
\hline 583 & 656 & $2^{4} \times 41$ & $1 \mathrm{~b}$ & $5^{2} 2^{4} 41^{4}$ & 3 & 2 & 2 & 4 & 1 & $(-,-, \otimes,-)$ & $\alpha_{2}$ & $\mathcal{K}, \mathfrak{K}_{1}$ \\
\hline 584 & 657 & $3^{2} \times 73$ & 2 & $3^{4} 73^{4}$ & 1 & 0 & 0 & 0 & 5 & $(\times,-,-,-)$ & $\varepsilon$ & \\
\hline 585 & 658 & $2 \times 7 \times 47$ & $1 \mathrm{~b}$ & $5^{2} 2^{4} 7^{4} 47^{4}$ & 12 & 1 & 2 & 5 & 6 & $(\times,-,-,-)$, & $\gamma$ & $2 \times 5,47 \times 5$ \\
\hline 586 & 659 & & $1 \mathrm{~b}$ & $5^{2} 659^{4}$ & 1 & 1 & 1 & 2 & 3 & $(-, \otimes,-,-)$ & $\delta_{2}$ & $\mathcal{L}$ \\
\hline 587 & ${ }^{*} 660$ & $2^{2} \times 3 \times 5 \times 11$ & $1 \mathrm{a}$ & $5^{6} 2^{4} 3^{4} 11^{4}$ & 64 & 1 & 2 & 5 & 6 & $(-,-, \times,-)$ & $\gamma$ & $2 \times 5,5 \times 11$ \\
\hline 588 & 661 & & $1 \mathrm{~b}$ & $5^{2} 661^{4}$ & 1 & 1 & 1 & 2 & 3 & $(-,-, \otimes,-)$ & $\alpha_{2}$ & $\mathcal{L}, \mathfrak{K}_{1}$ \\
\hline 589 & 662 & $2 \times 331$ & $1 \mathrm{~b}$ & $5^{2} 2^{4} 331^{4}$ & 3 & 1 & 1 & 3 & 4 & $(-,-,(\times),-)$ & $\beta_{2}$ & $2^{4} \times 5, \mathcal{K}$ \\
\hline 590 & 663 & $3 \times 13 \times 17$ & $1 \mathrm{~b}$ & $5^{2} 3^{4} 13^{4} 17^{4}$ & 13 & 1 & 2 & 5 & 6 & $(\times,-,-,-)$ & $\gamma$ & $3^{2} \times 5,13^{2} \times 5$ \\
\hline 591 & 664 & $2^{3} \times 83$ & $1 \mathrm{~b}$ & $5^{2} 2^{4} 83^{4}$ & 3 & 1 & 2 & 4 & 5 & $(\times,-,-,-)$ & $\varepsilon$ & $2^{2} \times 5$ \\
\hline 592 & *665 & $5 \times 7 \times 19$ & 1a & $5^{6} 7^{4} 19^{4}$ & 16 & 1 & 1 & 3 & 4 & $(-, \otimes,-,-)$ & $\beta_{2}$ & $7, \mathcal{K}$ \\
\hline 593 & 666 & $2 \times 3^{2} \times 37$ & $1 \mathrm{~b}$ & $5^{2} 2^{4} 3^{4} 37^{4}$ & 13 & 1 & 2 & 5 & 6 & $(\times,-,-,-)$ & $\gamma$ & $37,2 \times 5^{2}$ \\
\hline 594 & 667 & $23 \times 29$ & $1 \mathrm{~b}$ & $5^{2} 23^{4} 29^{4}$ & 3 & 1 & 1 & 3 & 4 & $(-, \otimes,-,-)$ & $\beta_{2}$ & $23 \times 5^{2}, \mathcal{K}$ \\
\hline 595 & 668 & $2^{2} \times 167$ & 2 & $2^{4} 167^{4}$ & 1 & 0 & 0 & 0 & 5 & $(\times,-,-,-)$ & $\varepsilon$ & \\
\hline 596 & 669 & $3 \times 223$ & $1 \mathrm{~b}$ & $5^{2} 3^{4} 223^{4}$ & 3 & 0 & 0 & 1 & 6 & $(\times,-,-,-)$ & $\gamma$ & \\
\hline 597 & 670 & $2 \times 5 \times 67$ & $1 \mathrm{a}$ & $5^{6} 2^{4} 67^{4}$ & 16 & 1 & 2 & 4 & 5 & $(\times,-,-,-)$ & $\varepsilon$ & 2 \\
\hline 598 & ${ }^{*} 671$ & $11 \times 61$ & $1 \mathrm{~b}$ & $5^{2} 11^{4} 61^{4}$ & 3 & 3 & 4 & 8 & 1 & $(-,-, \otimes,-)$ & $\alpha_{2}$ & $\begin{array}{l}\mathcal{K}_{(11)} \mathcal{K}_{(61)}, \\
\mathfrak{K}_{(11), 2} \mathfrak{K}_{(61), 1}^{2}\end{array}$ \\
\hline 599 & 673 & & $1 \mathrm{~b}$ & $5^{2} 673^{4}$ & 1 & 0 & 0 & 0 & 5 & $(\times,-,-,-)$ & $\varepsilon$ & \\
\hline 600 & 674 & $2 \times 337$ & 2 & $2^{4} 337^{4}$ & 1 & 0 & 0 & 0 & 5 & $(\times,-,-,-)$ & $\varepsilon$ & \\
\hline 601 & 677 & & $1 \mathrm{~b}$ & $5^{2} 677^{4}$ & 1 & 0 & 0 & 0 & 5 & $(\times,-,-,-)$ & $\varepsilon$ & \\
\hline 602 & 678 & $2 \times 3 \times 113$ & $1 \mathrm{~b}$ & $5^{2} 2^{4} 3^{4} 113^{4}$ & 13 & 1 & 2 & 5 & 6 & $(\times,-,-,-)$ & $\gamma$ & $2^{2} \times 5,3^{4} \times 5$ \\
\hline 603 & 679 & $7 \times 97$ & $1 b$ & $5^{2} 7^{4} 97^{4}$ & 4 & 0 & 0 & 1 & 6 & $(\times,-,-,--)$ & $\gamma$ & \\
\hline
\end{tabular}




\section{Continued}

\begin{tabular}{|c|c|c|c|c|c|c|c|c|c|c|c|c|}
\hline 604 & 680 & $2^{3} \times 5 \times 17$ & $1 \mathrm{a}$ & $5^{6} 2^{4} 17^{4}$ & 16 & 0 & 0 & 1 & 6 & $(\times,-,-,-)$ & $\gamma$ & \\
\hline 605 & 681 & $3 \times 227$ & $1 b$ & $5^{2} 3^{4} 227^{4}$ & 3 & 0 & 0 & 1 & 6 & $(\times,-,-,--)$ & $\gamma$ & \\
\hline 606 & ${ }^{*} 682$ & $2 \times 11 \times 31$ & 2 & $2^{4} 11^{4} 31^{4}$ & 3 & 2 & 3 & 6 & 3 & $(-,-, \otimes,-)$ & $\alpha_{2}$ & $\begin{array}{l}\mathcal{K}_{(11)} \mathcal{K}_{(31)}^{3}, \\
\mathfrak{K}_{(11), 1} \mathfrak{K}_{(31), 2}^{3}\end{array}$ \\
\hline 607 & 683 & & $1 b$ & $5^{2} 683^{4}$ & 1 & 0 & 0 & 0 & 5 & $(\times,-,-,-)$ & $\varepsilon$ & \\
\hline 608 & 684 & $2^{2} \times 3^{2} \times 19$ & $1 b$ & $5^{2} 2^{4} 3^{4} 19^{4}$ & 13 & 1 & 2 & 5 & 6 & $(-, \times,-,-)$ & $\gamma$ & $19 \times 5,2 \times 5^{3}$ \\
\hline 609 & 685 & $5 \times 137$ & $1 \mathrm{a}$ & $5^{6} 137^{4}$ & 4 & 0 & 0 & 0 & 5 & $(\times,-,-,-)$ & $\varepsilon$ & \\
\hline 610 & 687 & $3 \times 229$ & $1 b$ & $5^{2} 3^{4} 229^{4}$ & 3 & 1 & 1 & 3 & 4 & $(-, \otimes,-,-)$ & $\beta_{2}$ & $3 \times 5^{2}, \mathcal{K}$ \\
\hline 611 & 688 & $2^{4} \times 43$ & $1 b$ & $5^{2} 2^{4} 43^{4}$ & 4 & 0 & 0 & 1 & 6 & $(\times,-,-,-)$ & $\gamma$ & \\
\hline 612 & 689 & $13 \times 53$ & $1 b$ & $5^{2} 13^{4} 53^{4}$ & 3 & 0 & 0 & 1 & 6 & $(\times,-,-,-)$ & $\gamma$ & \\
\hline 613 & 690 & $2 \times 3 \times 5 \times 23$ & $1 \mathrm{a}$ & $5^{6} 2^{4} 3^{4} 23^{4}$ & 64 & 1 & 2 & 5 & 6 & $(\times,-,-,-)$ & $\gamma$ & $2,5 \times 23^{2}$ \\
\hline 614 & $* 691$ & & $1 b$ & $5^{2} 691^{4}$ & 1 & 3 & 4 & 8 & 1 & $(-,-, \otimes,-)$ & $\alpha_{2}$ & $\mathcal{L}, \mathfrak{K}_{2}$ \\
\hline 615 & 692 & $2^{2} \times 173$ & $1 b$ & $5^{2} 2^{4} 173^{4}$ & 3 & 1 & 2 & 4 & 5 & $(\times,-,-,-)$ & $\varepsilon$ & $2 \times 5$ \\
\hline 616 & *693 & $3^{2} \times 7 \times 11$ & 2 & $3^{4} 7^{4} 11^{4}$ & 4 & 2 & 3 & 6 & 3 & $(-,-, \otimes,-)$ & $\beta_{1}$ & $3 \times 7, \mathfrak{K}_{2}$ \\
\hline 617 & 694 & $2 \times 347$ & $1 b$ & $5^{2} 2^{4} 347^{4}$ & 3 & 0 & 0 & 1 & 6 & $(\times,-,-,-)$ & $\gamma$ & \\
\hline 618 & $* 695$ & $5 \times 139$ & $1 \mathrm{a}$ & $5^{6} 139^{4}$ & 4 & 1 & 2 & 4 & 5 & $(-, \times,-,-)$ & $\varepsilon$ & 139 \\
\hline 619 & 696 & $2^{3} \times 3 \times 29$ & $1 b$ & $5^{2} 2^{4} 3^{4} 29^{4}$ & 13 & 1 & 2 & 5 & 6 & $(-, \times,-,-)$ & $\gamma$ & $5,2 \times 29$ \\
\hline 620 & 697 & $17 \times 41$ & $1 b$ & $5^{2} 17^{4} 41^{4}$ & 3 & 1 & 1 & 3 & 4 & $(-,-,(\times),-)$ & $\beta_{2}$ & $17 \times 5, \mathcal{K}$ \\
\hline 621 & 698 & $2 \times 349$ & $1 \mathrm{~b}$ & $5^{2} 2^{4} 349^{4}$ & 4 & 1 & 1 & 3 & 4 & $(-, \otimes,-,-)$ & $\beta_{2}$ & $2^{4} \times 5, \mathcal{K}$ \\
\hline 622 & 699 & $3 \times 233$ & 2 & $3^{4} 233^{4}$ & 1 & 0 & 0 & 0 & 5 & $(\times,-,-,-)$ & $\varepsilon$ & \\
\hline 623 & 700 & $2^{2} \times 5^{2} \times 7$ & 1a & $5^{6} 2^{4} 7^{4}$ & 16 & 0 & 0 & 1 & 6 & $(\times,-,-,-)$ & $\gamma$ & \\
\hline 624 & 701 & & 2 & $701^{4}$ & 1 & 2 & 3 & 5 & 2 & $(-,-, \otimes, \times)$ & $\alpha_{1}$ & $\mathfrak{K}_{1}, \mathfrak{K}_{2}$ \\
\hline
\end{tabular}

Table 20. 47 pure metacyclic fields with normalized radicands $702 \leq D \leq 753$.

\begin{tabular}{ccccccccccccc}
\hline No. & $D$ & Factors & $\mathrm{S}$ & $f^{4}$ & $m$ & $V_{L}$ & $V_{M}$ & $V_{N}$ & $E$ & $(1,2,4,5)$ & $\mathrm{T}$ & $\mathrm{P}$ \\
\hline 625 & ${ }^{*} 702$ & $2 \times 3^{3} \times 13$ & $1 \mathrm{~b}$ & $5^{2} 2^{4} 3^{4} 13^{4}$ & 13 & 2 & 4 & 8 & 5 & $(\times,-,-,-)$ & $\varepsilon$ & $13 \times 5^{4}$ \\
626 & 703 & $19 \times 37$ & $1 \mathrm{~b}$ & $5^{2} 19^{4} 37^{4}$ & 3 & 1 & 1 & 3 & 4 & $(-, \otimes,-,-)$ & $\beta_{2}$ & $19 \times 5^{2}, \mathcal{K}$ \\
627 & 705 & $3 \times 5 \times 47$ & $1 \mathrm{a}$ & $5^{6} 3^{4} 47^{4}$ & 16 & 0 & 0 & 1 & 6 & $(\times,-,-,-)$ & $\gamma$ & \\
628 & 706 & $2 \times 353$ & $1 \mathrm{~b}$ & $5^{2} 2^{4} 353^{4}$ & 3 & 0 & 0 & 1 & 6 & $(\times,-,-,-)$ & $\gamma$ & \\
629 & $* 707$ & $7 \times 101$ & 2 & $7^{4} 101^{4}$ & 4 & 1 & 1 & 3 & 4 & $(-,-,(\times), \otimes)$ & $\zeta_{2}$ & $\mathcal{K}$ \\
630 & 708 & $2^{2} \times 3 \times 59$ & $1 \mathrm{~b}$ & $5^{2} 2^{4} 3^{4} 59^{4}$ & 13 & 1 & 2 & 5 & 6 & $(-, \times,-,-)$ & $\gamma$ & $3 \times 5,59 \times 5$ \\
631 & 709 & & $1 \mathrm{~b}$ & $5^{2} 709^{4}$ & 1 & 1 & 1 & 2 & 3 & $(-, \otimes,-,-)$ & $\delta_{2}$ & $\mathcal{L}$ \\
632 & $* 710$ & $2 \times 5 \times 71$ & $1 \mathrm{a}$ & $5^{6} 2^{4} 71^{4}$ & 16 & 2 & 2 & 4 & 1 & $(-,-, \otimes,-)$ & $\alpha_{2}$ & $\mathcal{K}, \mathfrak{K}_{2}$ \\
633 & 711 & $3^{2} \times 79$ & $1 \mathrm{~b}$ & $5^{2} 3^{4} 79^{4}$ & 3 & 1 & 1 & 3 & 4 & $(-, \otimes,-,-)$ & $\beta_{2}$ & $5, \mathcal{K}$ \\
634 & 712 & $2^{3} \times 89$ & $1 \mathrm{~b}$ & $5^{2} 2^{4} 89^{4}$ & 3 & 1 & 2 & 4 & 5 & $(-, \times,-,-)$ & $\varepsilon$ & $2^{2} \times 5$ \\
635 & 713 & $23 \times 31$ & $1 \mathrm{~b}$ & $5^{2} 23^{4} 31^{4}$ & 3 & 1 & 1 & 3 & 4 & $(-,-,(\times),-)$ & $\beta_{2}$ & $5, \mathcal{K}$ \\
636 & 714 & $2 \times 3 \times 7 \times 17$ & $1 \mathrm{~b}$ & $5^{2} 2^{4} 3^{4} 7^{4} 17^{4}$ & 52 & 2 & 4 & 9 & 6 & $(\times,-,-,-)$ & $\gamma$ & $17 \times 5^{3}$, \\
\hline
\end{tabular}




\section{Continued}

\begin{tabular}{|c|c|c|c|c|c|c|c|c|c|c|c|c|}
\hline 637 & 715 & $5 \times 11 \times 13$ & $1 \mathrm{a}$ & $5^{6} 11^{4} 13^{4}$ & 16 & 1 & 1 & 3 & 4 & $(-,-,(\times),-)$ & $\beta_{2}$ & $11, \mathcal{L}$ \\
\hline 638 & 716 & $2^{2} \times 179$ & $1 \mathrm{~b}$ & $5^{2} 2^{4} 179^{4}$ & 3 & 1 & 1 & 3 & 4 & $(-, \otimes,-,-)$ & $\beta_{2}$ & $179, \mathcal{L}$ \\
\hline 639 & 717 & $3 \times 239$ & $1 \mathrm{~b}$ & $5^{2} 3^{4} 239^{4}$ & 3 & 1 & 1 & 3 & 4 & $(-, \otimes,-,-)$ & $\beta_{2}$ & $3^{2} \times 5, \mathcal{K}$ \\
\hline 640 & 718 & $2 \times 359$ & 2 & $2^{4} 359^{4}$ & 1 & 1 & 1 & 2 & 3 & $(-, \otimes,-,-)$ & $\delta_{2}$ & $\mathcal{K}$ \\
\hline 641 & 719 & & $1 \mathrm{~b}$ & $5^{2} 719^{4}$ & 1 & 1 & 1 & 2 & 3 & $(-, \otimes,-,-)$ & $\delta_{2}$ & $\mathcal{L}$ \\
\hline 642 & 720 & $2^{4} \times 3^{2} \times 5$ & 1a & $5^{6} 2^{4} 3^{4}$ & 16 & 0 & 0 & 1 & 6 & $(\times,-,-,-)$ & $\gamma$ & \\
\hline 643 & 721 & $7 \times 103$ & $1 \mathrm{~b}$ & $5^{2} 7^{4} 103^{4}$ & 4 & 0 & 0 & 1 & 6 & $(\times,-,-,-)$ & $\gamma$ & \\
\hline 644 & 723 & $3 \times 241$ & $1 \mathrm{~b}$ & $5^{2} 3^{4} 241^{4}$ & 3 & 1 & 1 & 3 & 4 & $(-,-,(\times),-)$ & $\beta_{2}$ & $3 \times 5, \mathcal{K}$ \\
\hline 645 & 724 & $2^{2} \times 181$ & 2 & $2^{4} 181^{4}$ & 1 & 1 & 1 & 2 & 3 & $(-,-, \otimes,-)$ & $\alpha_{2}$ & $\mathcal{K}, \mathfrak{K}_{1}$ \\
\hline 646 & 725 & $5^{2} \times 29$ & 1a & $5^{6} 29^{4}$ & 4 & 1 & 1 & 2 & 3 & $(-, \otimes,-,-)$ & $\delta_{2}$ & $\mathcal{K}$ \\
\hline 647 & 726 & $2 \times 3 \times 11^{2}$ & 2 & $2^{4} 3^{4} 11^{4}$ & 3 & 1 & 1 & 3 & 4 & $(-,-,(\times),-)$ & $\beta_{2}$ & $2^{2} \times 3, \mathcal{K}$ \\
\hline 648 & 727 & & $1 b$ & $5^{2} 727^{4}$ & 1 & 0 & 0 & 0 & 5 & $(\times,-,-,-)$ & $\varepsilon$ & \\
\hline 649 & 728 & $2^{3} \times 7 \times 13$ & $1 \mathrm{~b}$ & $5^{2} 2^{4} 7^{4} 13^{4}$ & 12 & 1 & 2 & 5 & 6 & $(\times,-,-,-)$ & $\gamma$ & 5,7 \\
\hline 650 & 730 & $2 \times 5 \times 73$ & la & $5^{6} 2^{4} 73^{4}$ & 16 & 0 & 0 & 1 & 6 & $(\times,-,-,--)$ & $\gamma$ & \\
\hline 651 & 731 & $17 \times 43$ & $1 \mathrm{~b}$ & $5^{2} 17^{4} 43^{4}$ & 4 & 0 & 0 & 1 & 6 & $(\times,-,-,-)$ & $\gamma$ & \\
\hline 652 & 732 & $2^{2} \times 3 \times 61$ & 2 & $2^{4} 3^{4} 61^{4}$ & 3 & 1 & 1 & 3 & 4 & $(-,-,(\times),-)$ & $\beta_{2}$ & $2^{3} \times 3^{2}, \mathcal{K}$ \\
\hline 653 & 733 & & $1 \mathrm{~b}$ & $5^{2} 733^{4}$ & 1 & 0 & 0 & 0 & 5 & $(\times,-,-,-)$ & $\varepsilon$ & \\
\hline 654 & 734 & $2 \times 367$ & $1 \mathrm{~b}$ & $5^{2} 2^{4} 367^{4}$ & 3 & 0 & 0 & 1 & 6 & $(\times,-,-,-)$ & $\gamma$ & \\
\hline 655 & 735 & $3 \times 5 \times 7^{2}$ & 1a & $5^{6} 3^{4} 7^{4}$ & 16 & 0 & 0 & 1 & 6 & $(\times,-,-,-)$ & $\gamma$ & \\
\hline 656 & 737 & $11 \times 67$ & $1 \mathrm{~b}$ & $5^{2} 11^{4} 67^{4}$ & 3 & 1 & 2 & 4 & 5 & $(-,-, \otimes,-)$ & $\beta_{1}$ & $11, \mathfrak{K}_{1}$ \\
\hline 657 & 738 & $2 \times 3^{2} \times 41$ & $1 b$ & $5^{2} 2^{4} 3^{4} 41^{4}$ & 13 & 1 & 2 & 5 & 6 & $(-,-, \times,-)$ & $\gamma$ & $3,2 \times 5^{2}$ \\
\hline 658 & 739 & & $1 \mathrm{~b}$ & $5^{2} 739^{4}$ & 1 & 1 & 1 & 2 & 3 & $(-, \otimes,-,-)$ & $\delta_{2}$ & $\mathcal{L}$ \\
\hline 659 & 740 & $2^{2} \times 5 \times 37$ & 1a & $5^{6} 2^{4} 37^{4}$ & 16 & 1 & 2 & 4 & 5 & $(\times,-,-,--)$ & $\varepsilon$ & 37 \\
\hline 660 & 741 & $3 \times 13 \times 19$ & $1 \mathrm{~b}$ & $5^{2} 3^{4} 13^{4} 19^{4}$ & 13 & 1 & 2 & 5 & 6 & $(-, \times,-,-)$ & $\gamma$ & $3 \times 5^{2}, 19 \times 5$ \\
\hline 661 & 742 & $2 \times 7 \times 53$ & $1 \mathrm{~b}$ & $5^{2} 2^{4} 7^{4} 53^{4}$ & 12 & 1 & 2 & 5 & 6 & $(\times,-,-,-)$ & $\gamma$ & $2 \times 5^{2}, 7 \times 5^{2}$ \\
\hline 662 & 743 & & 2 & $743^{4}$ & 1 & 0 & 0 & 0 & 5 & $(-,-,-, \otimes)$ & $\vartheta$ & \\
\hline 663 & 744 & $2^{3} \times 3 \times 31$ & $1 \mathrm{~b}$ & $5^{2} 2^{4} 3^{4} 31^{4}$ & 13 & 1 & 2 & 5 & 6 & $(-,-, \times,-)$ & $\gamma$ & $5,3 \times 31^{2}$ \\
\hline 664 & $\star 745$ & $5 \times 149$ & la & $5^{6} 149^{4}$ & 4 & 1 & 1 & 3 & 4 & $(-, \otimes,-, \otimes)$ & $\zeta_{2}$ & $\mathcal{K}$ \\
\hline 665 & 746 & $2 \times 373$ & $1 \mathrm{~b}$ & $5^{2} 2^{4} 373^{4}$ & 3 & 1 & 2 & 4 & 5 & $(\times,-,-,-)$ & $\varepsilon$ & $2^{4} \times 5$ \\
\hline 666 & 747 & $3^{2} \times 83$ & $1 \mathrm{~b}$ & $5^{2} 3^{4} 83^{4}$ & 3 & 0 & 0 & 1 & 6 & $(\times,-,-,-)$ & $\gamma$ & \\
\hline 667 & 748 & $2^{2} \times 11 \times 17$ & $1 b$ & $5^{2} 2^{4} 11^{4} 17^{4}$ & 13 & 1 & 2 & 5 & 6 & $(-,-, \times,-)$ & $\gamma$ & $2 \times 5,2 \times 17$ \\
\hline 668 & ${ }^{*} 749$ & $7 \times 107$ & 2 & $7^{4} 107^{4}$ & 4 & 1 & 2 & 4 & 5 & $(-,-,-, \times)$ & $\varepsilon$ & \\
\hline 669 & $\star 751$ & & 2 & $751^{4}$ & 1 & 2 & 2 & 4 & 1 & $(-,-, \otimes, \times)$ & $\alpha_{2}$ & $\mathcal{L}, \mathfrak{K}_{1}$ \\
\hline 670 & 752 & $2^{4} \times 47$ & $1 \mathrm{~b}$ & $5^{2} 2^{4} 47^{4}$ & 3 & 0 & 0 & 1 & 6 & $(\times,-,-,-)$ & $\gamma$ & \\
\hline 671 & 753 & $3 \times 251$ & $1 \mathrm{~b}$ & $5^{2} 3^{4} 251^{4}$ & 4 & 1 & 1 & 3 & 4 & $(-,-,(\times),-)$ & $\beta_{2}$ & $5, \mathcal{K}$ \\
\hline
\end{tabular}


Table 21. 44 pure metacyclic fields with normalized radicands $754 \leq D \leq 799$.

\begin{tabular}{|c|c|c|c|c|c|c|c|c|c|c|c|c|}
\hline No. & $D$ & Factors & $S$ & $f^{*}$ & $m$ & $V_{L}$ & $V_{M}$ & $V_{N}$ & $E$ & $(1,2,4,5)$ & $\mathrm{T}$ & $\mathrm{P}$ \\
\hline 672 & 754 & $2 \times 13 \times 29$ & $1 b$ & $5^{2} 2^{4} 13^{4} 29^{4}$ & 13 & 1 & 2 & 5 & 6 & $(-, \times,-,-)$ & $\gamma$ & $13 \times 5,29 \times 5$ \\
\hline 673 & 755 & $5 \times 151$ & 1a & $5^{6} 151^{4}$ & 4 & 1 & 1 & 3 & 4 & $(-,-,(\times), \otimes)$ & $\zeta_{2}$ & $\mathcal{K}$ \\
\hline 674 & 756 & $2^{2} \times 3^{3} \times 7$ & $1 \mathrm{~b}$ & $5^{2} 2^{4} 3^{4} 7^{4}$ & 12 & 1 & 2 & 5 & 6 & $(\times,-,-,--)$ & $\gamma$ & $7,2^{3} \times 5^{2}$ \\
\hline 675 & 757 & & 2 & $757^{4}$ & 1 & 0 & 0 & 0 & 5 & $(-,-,-, \otimes)$ & $\vartheta$ & \\
\hline 676 & 758 & $2 \times 379$ & $1 b$ & $5^{2} 2^{4} 379^{4}$ & 3 & 1 & 1 & 3 & 4 & $(-, \otimes,-,-)$ & $\beta_{2}$ & $2 \times 5^{2}, \mathcal{K}$ \\
\hline 677 & 759 & $3 \times 11 \times 23$ & $1 b$ & $5^{2} 3^{4} 11^{4} 23^{4}$ & 13 & 2 & 3 & 6 & 3 & $(-,-, \otimes,-)$ & $\beta_{1}$ & $3^{2} \times 23 \times 5, \mathfrak{K}_{2}$ \\
\hline 678 & 760 & $2^{3} \times 5 \times 19$ & $1 \mathrm{a}$ & $5^{6} 2^{4} 19^{4}$ & 16 & 1 & 1 & 3 & 4 & $(-, \otimes,-,-)$ & $\beta_{2}$ & $2^{4} \times 19, \mathcal{K}$ \\
\hline 679 & 761 & & $1 b$ & $5^{2} 761^{4}$ & 1 & 2 & 3 & 5 & 2 & $(-,-, \otimes,-)$ & $\alpha_{1}$ & $\mathfrak{K}_{1}, \mathfrak{K}_{2}$ \\
\hline 680 & 762 & $2 \times 3 \times 127$ & $1 \mathrm{~b}$ & $5^{2} 2^{4} 3^{4} 127^{4}$ & 13 & 1 & 2 & 5 & 6 & $(\times,-,-,-)$ & $\gamma$ & $127 \times 5,3 \times 5^{3}$ \\
\hline 681 & 763 & $7 \times 109$ & $1 \mathrm{~b}$ & $5^{2} 7^{4} 109^{4}$ & 4 & 1 & 1 & 3 & 4 & $(-, \otimes,-,-)$ & $\beta_{2}$ & $7 \times 5^{2}, \mathcal{K}$ \\
\hline 682 & 764 & $2^{2} \times 191$ & $1 \mathrm{~b}$ & $5^{2} 2^{4} 191^{4}$ & 3 & 2 & 2 & 4 & 1 & $(-,-, \otimes,-)$ & $\alpha_{2}$ & $\mathcal{K}, \mathfrak{K}_{2}$ \\
\hline 683 & 765 & $3^{2} \times 5 \times 17$ & 1a & $5^{6} 3^{4} 17^{4}$ & 16 & 0 & 0 & 1 & 6 & $(\times,-,-,-)$ & $\gamma$ & \\
\hline 684 & 766 & $2 \times 383$ & $1 \mathrm{~b}$ & $5^{2} 2^{4} 383^{4}$ & 3 & 0 & 0 & 1 & 6 & $(\times,-,-,--)$ & $\gamma$ & \\
\hline 685 & 767 & $13 \times 59$ & $1 \mathrm{~b}$ & $5^{2} 13^{4} 59^{4}$ & 3 & 1 & 1 & 3 & 4 & $(-, \otimes,-,-)$ & $\beta_{2}$ & $13 \times 5^{2}, \mathcal{K}$ \\
\hline 686 & 769 & & $1 \mathrm{~b}$ & $5^{2} 769^{4}$ & 1 & 1 & 1 & 2 & 3 & $(-, \otimes,-,-)$ & $\delta_{2}$ & $\mathcal{L}$ \\
\hline 687 & $\star 770$ & $2 \times 5 \times 7 \times 11$ & $1 \mathrm{a}$ & $5^{6} 2^{4} 7^{4} 11^{4}$ & 64 & 1 & 2 & 5 & 6 & $(-,-, \times,-)$ & $\gamma$ & $2 \times 5,2^{2} \times 11$ \\
\hline 688 & 771 & $3 \times 257$ & $1 \mathrm{~b}$ & $5^{2} 3^{4} 257^{4}$ & 4 & 0 & 0 & 1 & 6 & $(\times,-,-,-)$ & $\gamma$ & \\
\hline 689 & 772 & $2^{2} \times 193$ & $1 \mathrm{~b}$ & $5^{2} 2^{4} 193^{4}$ & 4 & 1 & 2 & 4 & 5 & $(\times,-,-,-)$ & $\varepsilon$ & 2 \\
\hline 690 & 773 & & $1 \mathrm{~b}$ & $5^{2} 773^{4}$ & 1 & 0 & 0 & 0 & 5 & $(\times,-,-,-)$ & $\varepsilon$ & \\
\hline 691 & 774 & $2 \times 3^{2} \times 43$ & 2 & $2^{4} 3^{4} 43^{4}$ & 4 & 0 & 0 & 1 & 6 & $(\times,-,-,-)$ & $\gamma$ & \\
\hline 692 & 775 & $5^{2} \times 31$ & 1a & $5^{6} 31^{4}$ & 4 & 2 & 3 & 5 & 2 & $(-,-, \otimes,-)$ & $\alpha_{1}$ & $\mathfrak{K}_{1}, \mathfrak{K}_{2}$ \\
\hline 693 & 776 & $2^{3} \times 97$ & 2 & $2^{4} 97^{4}$ & 1 & 0 & 0 & 0 & 5 & $(\times,-,-,-)$ & $\varepsilon$ & \\
\hline 694 & 777 & $3 \times 7 \times 37$ & $1 b$ & $5^{2} 3^{4} 7^{4} 37^{4}$ & 12 & 1 & 2 & 5 & 6 & $(\times,-,-,-)$ & $\gamma$ & $3,7 \times 5$ \\
\hline 695 & 778 & $2 \times 389$ & $1 \mathrm{~b}$ & $5^{2} 2^{4} 389^{4}$ & 3 & 1 & 1 & 3 & 4 & $(-, \otimes,-,-)$ & $\beta_{2}$ & $5, \mathcal{K}$ \\
\hline 696 & $\star 779$ & $19 \times 41$ & $1 \mathrm{~b}$ & $5^{2} 19^{4} 41^{4}$ & 3 & 3 & 4 & 8 & 1 & $(-, \otimes, \otimes,-)$ & $\alpha_{2}$ & $\mathcal{K}_{(19)} \mathcal{K}_{(41)}, \mathfrak{K}_{(41), !}$ \\
\hline 697 & 780 & $2^{2} \times 3 \times 5 \times 13$ & $1 \mathrm{a}$ & $5^{6} 2^{4} 3^{4} 13^{4}$ & 64 & 1 & 2 & 5 & 6 & $(\times,-,-,-)$ & $\gamma$ & $2 \times 5,5 \times 13$ \\
\hline 698 & 781 & $11 \times 71$ & $1 \mathrm{~b}$ & $5^{2} 11^{4} 71^{4}$ & 3 & 3 & 5 & 9 & 2 & $(-,-, \otimes,-)$ & $\alpha_{1}$ & $\begin{array}{l}\mathfrak{K}_{(11) !} \mathfrak{K}_{(71), 1}^{4}, \\
\mathfrak{K}_{(11), 2} \mathfrak{K}_{(71), 2}^{2}\end{array}$ \\
\hline 699 & 782 & $2 \times 17 \times 23$ & 2 & $2^{4} 17^{4} 23^{4}$ & 3 & 0 & 0 & 1 & 6 & $(\times,-,-,-)$ & $\gamma$ & \\
\hline 700 & 783 & $3^{3} \times 29$ & $1 \mathrm{~b}$ & $5^{2} 3^{4} 29^{4}$ & 3 & 1 & 1 & 3 & 4 & $(-, \otimes,-,-)$ & $\beta_{2}$ & $29, \mathcal{L}$ \\
\hline 701 & ${ }^{*} 785$ & $5 \times 157$ & 1a & $5^{6} 157^{4}$ & 4 & 1 & 2 & 4 & 5 & $(-,-,-, \times)$ & $\varepsilon$ & \\
\hline 702 & 786 & $2 \times 3 \times 131$ & $1 b$ & $5^{2} 2^{4} 3^{4} 131^{4}$ & 13 & 2 & 3 & 7 & 4 & $(-,-,(\times),-)$ & $\beta_{2}$ & $2 \times 3 \times 5^{2}, \mathcal{K}$ \\
\hline 703 & 787 & & $1 \mathrm{~b}$ & $5^{2} 787^{4}$ & 1 & 0 & 0 & 0 & 5 & $(\times,-,-,--)$ & $\varepsilon$ & \\
\hline 704 & 788 & $2^{2} \times 197$ & $1 \mathrm{~b}$ & $5^{2} 2^{4} 197^{4}$ & 3 & 0 & 0 & 1 & 6 & $(\times,-,-,-)$ & $\gamma$ & \\
\hline 705 & 789 & $3 \times 263$ & $1 \mathrm{~b}$ & $5^{2} 3^{4} 263^{4}$ & 3 & 0 & 0 & 1 & 6 & $(\times,-,-,-)$ & $\gamma$ & \\
\hline 706 & 790 & $2 \times 5 \times 79$ & $1 \mathrm{a}$ & $5^{6} 2^{4} 79^{4}$ & 16 & 1 & 2 & 4 & 5 & $(-, \times,-,-)$ & $\varepsilon$ & 5 \\
\hline 707 & 791 & $7 \times 113$ & $1 \mathrm{~b}$ & $5^{2} 7^{4} 113^{4}$ & 4 & 0 & 0 & 1 & 6 & $(\times,-,-,--)$ & $\gamma$ & \\
\hline
\end{tabular}




\section{Continued}

\begin{tabular}{ccccccccccccc}
\hline 708 & 792 & $2^{3} \times 3^{2} \times 11$ & $1 \mathrm{~b}$ & $5^{2} 2^{4} 3^{4} 11^{4}$ & 13 & 1 & 2 & 5 & 6 & $(-,-, \times,-)$ & $\gamma$ & $2 \times 5,11 \times 5$ \\
709 & 793 & $13 \times 61$ & 2 & $13^{4} 61^{4}$ & 1 & 1 & 2 & 4 & 5 & $(-,-, \otimes,-)$ & $\beta_{1}$ & $61, \mathfrak{K}_{2}$ \\
710 & 794 & $2 \times 397$ & $1 \mathrm{~b}$ & $5^{2} 2^{4} 397^{4}$ & 3 & 0 & 0 & 1 & 6 & $(\times,-,-,-)$ & $\gamma$ & \\
711 & 795 & $3 \times 5 \times 53$ & $1 \mathrm{a}$ & $5^{6} 3^{4} 53^{4}$ & 16 & 0 & 0 & 1 & 6 & $(\times,-,-,-)$ & $\gamma$ & \\
712 & 796 & $2^{2} \times 199$ & $1 \mathrm{~b}$ & $5^{2} 2^{4} 199^{4}$ & 4 & 1 & 1 & 3 & 4 & $(-, \otimes,-,-)$ & $\beta_{2}$ & $2 \times 5, \mathcal{K}$ \\
713 & 797 & & $1 \mathrm{~b}$ & $5^{2} 797^{4}$ & 1 & 0 & 0 & 0 & 5 & $(\times,-,-,-)$ & $\varepsilon$ & \\
714 & ${ }_{798}$ & $2 \times 3 \times 7 \times 19$ & $1 \mathrm{~b}$ & $5^{2} 2^{4} 3^{4} 7^{4} 19^{4}$ & 52 & 2 & 4 & 9 & 6 & $(-, \times,-,-)$ & $\gamma$ & $3 \times 19,7 \times 5^{4}$ \\
715 & 799 & $17 \times 47$ & 2 & $17^{4} 47^{4}$ & 1 & 0 & 0 & 0 & 5 & $(\times,-,-,-)$ & $\varepsilon$ & \\
\hline
\end{tabular}

Table 22. 46 pure metacyclic fields with normalized radicands $801 \leq D \leq 848$.

\begin{tabular}{|c|c|c|c|c|c|c|c|c|c|c|c|c|}
\hline No. & $D$ & Factors & $S$ & $f^{*}$ & $m$ & $V_{L}$ & $V_{M}$ & $V_{N}$ & $E$ & $(1,2,4,5)$ & $\mathrm{T}$ & $\mathrm{P}$ \\
\hline 716 & 801 & $3^{2} \times 89$ & 2 & $3^{4} 89^{4}$ & 1 & 1 & 1 & 2 & 3 & $(-, \otimes,-,-)$ & $\delta_{2}$ & $\mathcal{K}$ \\
\hline 717 & 802 & $2 \times 401$ & $1 \mathrm{~b}$ & $5^{2} 2^{4} 401^{4}$ & 4 & 1 & 1 & 3 & 4 & $(-,-,(\times),-)$ & $\beta_{2}$ & $2^{2} \times 5, \mathcal{K}$ \\
\hline 718 & 803 & $11 \times 73$ & $1 \mathrm{~b}$ & $5^{2} 11^{4} 73^{4}$ & 3 & 1 & 1 & 3 & 4 & $(-,-,(\times),-)$ & $\beta_{2}$ & $73 \times 5^{2}, \mathcal{K}$ \\
\hline 719 & 804 & $2^{2} \times 3 \times 67$ & $1 \mathrm{~b}$ & $5^{2} 2^{4} 3^{4} 67^{4}$ & 13 & 1 & 2 & 5 & 6 & $(\times,-,-,-)$ & $\gamma$ & $3,2 \times 5^{3}$ \\
\hline 720 & 805 & $5 \times 7 \times 23$ & $1 \mathrm{a}$ & $5^{6} 7^{4} 23^{4}$ & 16 & 0 & 0 & 1 & 6 & $(\times,-,-,-)$ & $\gamma$ & \\
\hline 721 & 806 & $2 \times 13 \times 31$ & $1 b$ & $5^{2} 2^{4} 13^{4} 31^{4}$ & 13 & 2 & 3 & 6 & 3 & $(-,-, \otimes,-)$ & $\beta_{1}$ & $31 \times 5^{2}, \mathfrak{K}_{2}$ \\
\hline 722 & 807 & $3 \times 269$ & 2 & $3^{4} 269^{4}$ & 1 & 1 & 1 & 2 & 3 & $(-, \otimes,-,-)$ & $\delta_{2}$ & $\mathcal{K}$ \\
\hline 723 & ${ }^{*} 808$ & $2^{3} \times 101$ & $1 \mathrm{~b}$ & $5^{2} 2^{4} 101^{4}$ & 4 & 2 & 2 & 4 & 1 & $(-,-, \otimes,-)$ & $\alpha_{2}$ & $\mathcal{K}, \mathfrak{K}_{1}$ \\
\hline 724 & 809 & & $1 b$ & $5^{2} 809^{4}$ & 1 & 1 & 1 & 2 & 3 & $(-, \otimes,-,-)$ & $\delta_{2}$ & $\mathcal{L}$ \\
\hline 725 & 810 & $2 \times 3^{4} \times 5$ & $1 \mathrm{a}$ & $5^{6} 2^{4} 3^{4}$ & 16 & 0 & 0 & 1 & 6 & $(\times,-,-,-)$ & $\gamma$ & \\
\hline 726 & 811 & & $1 \mathrm{~b}$ & $5^{2} 811^{4}$ & 1 & 1 & 1 & 2 & 3 & $(-,-, \otimes,-)$ & $\alpha_{2}$ & $\mathcal{L}, \mathfrak{K}_{1}$ \\
\hline 727 & 812 & $2^{2} \times 7 \times 29$ & $1 \mathrm{~b}$ & $5^{2} 2^{4} 7^{4} 29^{4}$ & 12 & 1 & 2 & 5 & 6 & $(-, \times,-,-)$ & $\gamma$ & $29,7^{2} \times 5$ \\
\hline 728 & 813 & $3 \times 271$ & $1 \mathrm{~b}$ & $5^{2} 3^{4} 271^{4}$ & 3 & 1 & 1 & 3 & 4 & $(-,-,(\times),-)$ & $\beta_{2}$ & $5, \mathcal{K}$ \\
\hline 729 & 814 & $2 \times 11 \times 37$ & $1 \mathrm{~b}$ & $5^{2} 2^{4} 11^{4} 37^{4}$ & 13 & 2 & 3 & 6 & 3 & $(-,-, \otimes,-)$ & $\beta_{1}$ & $11 \times 37^{2} \times 5, \mathfrak{K}_{2}$ \\
\hline 730 & 815 & $5 \times 163$ & 1a & $5^{6} 163^{4}$ & 4 & 0 & 0 & 0 & 5 & $(\times,-,-,--)$ & $\varepsilon$ & \\
\hline 731 & 816 & $2^{4} \times 3 \times 17$ & $1 \mathrm{~b}$ & $5^{2} 2^{4} 3^{4} 17^{4}$ & 13 & 1 & 2 & 5 & 6 & $(\times,-,-,-)$ & $\gamma$ & $2 \times 5^{2}, 3 \times 5^{3}$ \\
\hline 732 & 817 & $19 \times 43$ & $1 \mathrm{~b}$ & $5^{2} 19^{4} 43^{4}$ & 4 & 1 & 1 & 3 & 4 & $(-, \otimes,-,-)$ & $\beta_{2}$ & $19^{2} \times 5, \mathcal{K}$ \\
\hline 733 & 818 & $2 \times 409$ & 2 & $2^{4} 409^{4}$ & 1 & 1 & 1 & 2 & 3 & $(-, \otimes,-,--)$ & $\delta_{2}$ & $\mathcal{K}$ \\
\hline 734 & 819 & $3^{2} \times 7 \times 13$ & $1 \mathrm{~b}$ & $5^{2} 3^{4} 7^{4} 13^{4}$ & 12 & 1 & 2 & 5 & 6 & $(\times,-,-,-)$ & $\gamma$ & $13,7 \times 5^{2}$ \\
\hline 735 & 820 & $2^{2} \times 5 \times 41$ & la & $5^{6} 2^{4} 41^{4}$ & 16 & 2 & 2 & 4 & 1 & $(-,-, \otimes,-)$ & $\alpha_{2}$ & $\mathcal{K}, \mathfrak{K}_{1}$ \\
\hline 736 & 821 & & $1 \mathrm{~b}$ & $5^{2} 821^{4}$ & 1 & 1 & 1 & 2 & 3 & $(-,-, \otimes,-)$ & $\alpha_{2}$ & $\mathcal{L}, \mathfrak{K}_{2}$ \\
\hline 737 & 822 & $2 \times 3 \times 137$ & $1 \mathrm{~b}$ & $5^{2} 2^{4} 3^{4} 137^{4}$ & 13 & 2 & 4 & 8 & 5 & $(\times,-,-,-)$ & $\varepsilon$ & $3^{3} \times 137 \times 5$ \\
\hline 738 & 823 & & $1 b$ & $5^{2} 823^{4}$ & 1 & 0 & 0 & 0 & 5 & $(\times,-,-,-)$ & $\varepsilon$ & \\
\hline 739 & 824 & $2^{3} \times 103$ & 2 & $2^{4} 103^{4}$ & 1 & 0 & 0 & 0 & 5 & $(\times,-,-,-)$ & $\varepsilon$ & \\
\hline 740 & ${ }^{*} 825$ & $3 \times 5^{2} \times 11$ & $1 \mathrm{a}$ & $5^{6} 3^{4} 11^{4}$ & 16 & 1 & 2 & 4 & 5 & $(-,-, \otimes,-)$ & $\beta_{1}$ & $3^{2} \times 11, \mathfrak{K}_{1}$ \\
\hline 741 & 826 & $2 \times 7 \times 59$ & 2 & $2^{4} 7^{4} 59^{4}$ & 4 & 1 & 1 & 3 & 4 & $(-, \otimes,-,-)$ & $\beta_{2}$ & $59, \mathcal{L}$ \\
\hline
\end{tabular}




\section{Continued}

\begin{tabular}{|c|c|c|c|c|c|c|c|c|c|c|c|c|}
\hline 742 & 827 & & $1 \mathrm{~b}$ & $5^{2} 827^{4}$ & 1 & 0 & 0 & 0 & 5 & $(\times,-,-,-)$ & $\varepsilon$ & \\
\hline 743 & 828 & $2^{2} \times 3^{2} \times 23$ & $1 \mathrm{~b}$ & $5^{2} 2^{4} 3^{4} 23^{4}$ & 13 & 1 & 2 & 5 & 6 & $(\times,-,-,-)$ & $\gamma$ & $23,2 \times 5^{2}$ \\
\hline 744 & 829 & & $1 \mathrm{~b}$ & $5^{2} 829^{4}$ & 1 & 1 & 1 & 3 & 4 & $(-, \otimes,-,-)$ & $\beta_{2}$ & $829, \mathcal{L}$ \\
\hline 745 & 830 & $2 \times 5 \times 83$ & $1 \mathrm{a}$ & $5^{6} 2^{4} 83^{4}$ & 16 & 0 & 0 & 1 & 6 & $(\times,-,-,-)$ & $\gamma$ & \\
\hline 746 & 831 & $3 \times 277$ & $1 \mathrm{~b}$ & $5^{2} 3^{4} 277^{4}$ & 3 & 1 & 2 & 4 & 5 & $(\times,-,-,-)$ & $\varepsilon$ & $3^{2} \times 5$ \\
\hline 747 & 833 & $7^{2} \times 17$ & $1 \mathrm{~b}$ & $5^{2} 7^{4} 17^{4}$ & 4 & 0 & 0 & 1 & 6 & $(\times,-,-,-)$ & $\gamma$ & \\
\hline 748 & 834 & $2 \times 3 \times 139$ & $1 \mathrm{~b}$ & $5^{2} 2^{4} 3^{4} 139^{4}$ & 13 & 1 & 2 & 5 & 6 & $(-, \times,-,-)$ & $\gamma$ & $2 \times 5,3 \times 5^{3}$ \\
\hline 749 & 835 & $5 \times 167$ & $1 \mathrm{a}$ & $5^{6} 167^{4}$ & 4 & 0 & 0 & 0 & 5 & $(\times,-,-,-)$ & $\varepsilon$ & \\
\hline 750 & 836 & $2^{2} \times 11 \times 19$ & $1 \mathrm{~b}$ & $5^{2} 2^{4} 11^{4} 19^{4}$ & 13 & 2 & 3 & 7 & 4 & $(-, \otimes,(\times),-)$ & $\beta_{2}$ & $\begin{array}{l}2 \times 11 \times 5 \\
\mathcal{K}_{(11)} \times \mathcal{K}_{(19)}^{3}\end{array}$ \\
\hline 751 & 837 & $3^{3} \times 31$ & $1 \mathrm{~b}$ & $5^{2} 3^{4} 31^{4}$ & 3 & 1 & 1 & 3 & 4 & $(-,-,(\times),-)$ & $\beta_{2}$ & $5, \mathcal{K}$ \\
\hline 752 & 838 & $2 \times 419$ & $1 \mathrm{~b}$ & $5^{2} 2^{4} 419^{4}$ & 3 & 1 & 1 & 3 & 4 & $(-, \otimes,-,-)$ & $\beta_{2}$ & $2 \times 5, \mathcal{K}$ \\
\hline 753 & 839 & & $1 b$ & $5^{2} 839^{4}$ & 1 & 1 & 1 & 2 & 3 & $(-, \otimes,-,-)$ & $\delta_{2}$ & $\mathcal{L}$ \\
\hline 754 & 840 & $2^{3} \times 3 \times 5 \times 7$ & $1 \mathrm{a}$ & $5^{6} 2^{4} 3^{4} 7^{4}$ & 64 & 1 & 2 & 5 & 6 & $(\times,-,-,-)$ & $\gamma$ & $2 \times 7,3 \times 7$ \\
\hline 755 & 842 & $2 \times 421$ & $1 \mathrm{~b}$ & $5^{2} 2^{4} 421^{4}$ & 3 & 1 & 2 & 4 & 5 & $(-,-, \times,-)$ & $\varepsilon$ & $2 \times 5^{3}$ \\
\hline 756 & ${ }^{*} 843$ & $3 \times 281$ & 2 & $3^{4} 281^{4}$ & 1 & 1 & 2 & 3 & 4 & $(-,-, \otimes,-)$ & $\delta_{1}$ & $\mathfrak{K}_{1}$ \\
\hline 757 & 844 & $2^{2} \times 211$ & $1 \mathrm{~b}$ & $5^{2} 2^{4} 211^{4}$ & 3 & 1 & 2 & 4 & 5 & $(-,-, \times,-)$ & $\varepsilon$ & $2 \times 5^{2}$ \\
\hline 758 & 845 & $5 \times 13^{2}$ & $1 \mathrm{a}$ & $5^{6} 13^{4}$ & 4 & 0 & 0 & 0 & 5 & $(\times,-,-,-)$ & $\varepsilon$ & \\
\hline 759 & 846 & $2 \times 3^{2} \times 47$ & $1 \mathrm{~b}$ & $5^{2} 2^{4} 3^{4} 47^{4}$ & 13 & 1 & 2 & 5 & 6 & $(\times,-,-,-)$ & $\gamma$ & 2,3 \\
\hline 760 & 847 & $7 \times 11^{2}$ & $1 \mathrm{~b}$ & $5^{2} 7^{4} 11^{4}$ & 4 & 1 & 1 & 3 & 4 & $(-,-,(\times),-)$ & $\beta_{2}$ & $7 \times 5^{2}, \mathcal{K}$ \\
\hline 761 & 848 & $2^{4} \times 53$ & $1 b$ & $5^{2} 2^{4} 53^{4}$ & 3 & 0 & 0 & 1 & 6 & $(\times,-,-,-)$ & $\gamma$ & \\
\hline
\end{tabular}

Table 23. 47 pure metacyclic fields with normalized radicands $849 \leq D \leq 901$.

\begin{tabular}{ccccccccccccc}
\hline No. & $D$ & Factors & $\mathrm{S}$ & $f^{A}$ & $m$ & $V_{L}$ & $V_{M}$ & $V_{N}$ & $E$ & $(1,2,4,5)$ & $\mathrm{T}$ & $\mathrm{P}$ \\
\hline 762 & 849 & $3 \times 283$ & 2 & $3^{4} 283^{4}$ & 1 & 0 & 0 & 0 & 5 & $(\times,-,-,-)$ & $\varepsilon$ & \\
763 & 850 & $2 \times 5^{2} \times 17$ & $1 \mathrm{a}$ & $5^{6} 2^{4} 17^{4}$ & 16 & 0 & 0 & 1 & 6 & $(\times,-,-,-)$ & $\gamma$ & \\
764 & 851 & $23 \times 37$ & 2 & $23^{4} 37^{4}$ & 1 & 0 & 0 & 0 & 5 & $(\times,-,-,-)$ & $\varepsilon$ & \\
765 & 852 & $2^{2} \times 3 \times 71$ & $1 \mathrm{~b}$ & $5^{2} 2^{4} 3^{4} 71^{4}$ & 13 & 1 & 2 & 5 & 6 & $(-,-, \times,-)$ & $\gamma$ & $2 \times 5^{3}, 3 \times 5^{3}$ \\
766 & 853 & & $1 \mathrm{~b}$ & $5^{2} 853^{4}$ & 1 & 0 & 0 & 0 & 5 & $(\times,-,-,-)$ & $\varepsilon$ & \\
767 & 854 & $2 \times 7 \times 61$ & $1 \mathrm{~b}$ & $5^{2} 2^{4} 7^{4} 61^{4}$ & 12 & 1 & 2 & 5 & 6 & $(-,-, \times,-)$ & $\gamma$ & $2 \times 5^{2}, 61$ \\
768 & 855 & $3^{2} \times 5 \times 19$ & $1 \mathrm{a}$ & $5^{6} 3^{4} 19^{4}$ & 16 & 1 & 1 & 3 & 4 & $(-, \otimes,-,-)$ & $\beta_{2}$ & $3, \mathcal{K}$ \\
769 & 856 & $2^{3} \times 107$ & $1 \mathrm{~b}$ & $5^{2} 2^{4} 107^{4}$ & 4 & 0 & 0 & 1 & 6 & $(\times,-,-,-)$ & $\gamma$ & \\
770 & 857 & & 2 & $857^{4}$ & 1 & 0 & 0 & 0 & 5 & $(-,-,-, \otimes)$ & $\vartheta$ & \\
771 & $* 858$ & $2 \times 3 \times 11 \times 13$ & $1 \mathrm{~b}$ & $5^{2} 2^{4} 3^{4} 11^{4} 13^{4}$ & 51 & 2 & 4 & 9 & 6 & $(-,-, \times,-)$ & $\gamma$ & $2 \times 5,13 \times 5$ \\
772 & 859 & & $1 \mathrm{~b}$ & $5^{2} 859^{4}$ & 1 & 1 & 1 & 2 & 3 & $(-, \otimes,-,-)$ & $\delta_{2}$ & $\mathcal{L}$ \\
773 & 860 & $2^{2} \times 5 \times 43$ & $1 \mathrm{a}$ & $5^{6} 2^{4} 43^{4}$ & 16 & 0 & 0 & 1 & 6 & $(\times,-,-,-)$ & $\gamma$ & \\
774 & $* 861$ & $3 \times 7 \times 41$ & $1 \mathrm{~b}$ & $5^{2} 3^{4} 7^{4} 41^{4}$ & 12 & 3 & 4 & 8 & 1 & $(-,-, \otimes,-)$ & $\alpha_{2}$ & $\mathcal{K}, \mathfrak{K}_{1}$ \\
\hline
\end{tabular}




\section{Continued}

\begin{tabular}{|c|c|c|c|c|c|c|c|c|c|c|c|c|}
\hline 775 & 862 & $2 \times 431$ & $1 b$ & $5^{2} 2^{4} 431^{4}$ & 3 & 1 & 2 & 4 & 5 & $(-,-, \otimes,-)$ & $\beta_{1}$ & $431, \mathfrak{K}_{1}$ \\
\hline 776 & 863 & & $1 b$ & $5^{2} 863^{4}$ & 1 & 0 & 0 & 0 & 5 & $(\times,-,-,-)$ & $\varepsilon$ & \\
\hline 777 & 865 & $5 \times 173$ & 1a & $5^{6} 173^{4}$ & 4 & 0 & 0 & 0 & 5 & $(\times,-,-,-)$ & $\varepsilon$ & \\
\hline 778 & 866 & $2 \times 433$ & $1 b$ & $5^{2} 2^{4} 433^{4}$ & 3 & 0 & 0 & 1 & 6 & $(\times,-,-,-)$ & $\gamma$ & \\
\hline 779 & 868 & $2^{2} \times 7 \times 31$ & 2 & $2^{4} 7^{4} 31^{4}$ & 4 & 1 & 1 & 3 & 4 & $(-,-,(\times),-)$ & $\beta_{2}$ & $2 \times 7^{2}, \mathcal{K}$ \\
\hline 780 & 869 & $11 \times 79$ & $1 b$ & $5^{2} 11^{4} 79^{4}$ & 3 & 2 & 2 & 5 & 2 & $(-, \otimes,(\times),-)$ & $\alpha_{3}$ & $\mathcal{K}_{(11)}, \mathcal{K}_{(79)}$ \\
\hline 781 & 870 & $2 \times 3 \times 5 \times 29$ & 1a & $5^{6} 2^{4} 3^{4} 29^{4}$ & 64 & 1 & 2 & 5 & 6 & $(-, \times,-,-)$ & $\gamma$ & $29,3^{2} \times 5$ \\
\hline 782 & 871 & $13 \times 67$ & $1 b$ & $5^{2} 13^{4} 67^{4}$ & 3 & 0 & 0 & 1 & 6 & $(\times,-,-,-)$ & $\gamma$ & \\
\hline 783 & 872 & $2^{3} \times 109$ & $1 b$ & $5^{2} 2^{4} 109^{4}$ & 3 & 1 & 1 & 3 & 4 & $(-, \otimes,-,-)$ & $\beta_{2}$ & $109, \mathcal{L}$ \\
\hline 784 & 873 & $3^{2} \times 97$ & $1 b$ & $5^{2} 3^{4} 97^{4}$ & 3 & 0 & 0 & 1 & 6 & $(\times,-,-,-)$ & $\gamma$ & \\
\hline 785 & 874 & $2 \times 19 \times 23$ & 2 & $2^{4} 19^{4} 23^{4}$ & 3 & 1 & 1 & 3 & 4 & $(-, \otimes,-,-)$ & $\beta_{2}$ & $2 \times 19^{2}, \mathcal{K}$ \\
\hline 786 & 876 & $2^{2} \times 3 \times 73$ & 2 & $2^{4} 3^{4} 73^{4}$ & 3 & 0 & 0 & 1 & 6 & $(\times,-,-,-)$ & $\gamma$ & \\
\hline 787 & 877 & & $1 b$ & $5^{2} 877^{4}$ & 1 & 0 & 0 & 0 & 5 & $(\times,-,-,--)$ & $\varepsilon$ & \\
\hline 788 & 878 & $2 \times 439$ & $1 b$ & $5^{2} 2^{4} 439^{4}$ & 3 & 1 & 1 & 3 & 4 & $(-, \otimes,-,-)$ & $\beta_{2}$ & $5, \mathcal{K}$ \\
\hline 789 & 879 & $3 \times 293$ & $1 b$ & $5^{2} 3^{4} 293^{4}$ & 4 & 1 & 2 & 4 & 5 & $(\times,-,-,-)$ & $\varepsilon$ & 3 \\
\hline 790 & 880 & $2^{4} \times 5 \times 11$ & 1a & $5^{6} 2^{4} 11^{4}$ & 16 & 2 & 2 & 4 & 1 & $(-,-, \otimes,-)$ & $\alpha_{2}$ & $\mathcal{K}, \mathfrak{K}_{2}$ \\
\hline 791 & 881 & & $1 b$ & $5^{2} 881^{4}$ & 1 & 1 & 2 & 3 & 4 & $(-,-, \otimes,-)$ & $\delta_{1}$ & $\mathfrak{K}_{2}$ \\
\hline 792 & 883 & & $1 b$ & $5^{2} 883^{4}$ & 1 & 0 & 0 & 0 & 5 & $(\times,-,-,-)$ & $\varepsilon$ & \\
\hline 793 & 884 & $2^{2} \times 13 \times 17$ & $1 b$ & $5^{2} 2^{4} 13^{4} 17^{4}$ & 13 & 1 & 2 & 5 & 6 & $(\times,-,-,--)$ & $\gamma$ & $17,13 \times 5^{2}$ \\
\hline 794 & 885 & $3 \times 5 \times 59$ & 1a & $5^{6} 3^{4} 59^{4}$ & 16 & 1 & 1 & 3 & 4 & $(-, \otimes,-,-)$ & $\beta_{2}$ & $3, \mathcal{K}$ \\
\hline 795 & 886 & $2 \times 443$ & $1 b$ & $5^{2} 2^{4} 443^{4}$ & 4 & 0 & 0 & 1 & 6 & $(\times,-,-,-)$ & $\gamma$ & \\
\hline 796 & 887 & & $1 b$ & $5^{2} 887^{4}$ & 1 & 0 & 0 & 0 & 5 & $(\times,-,-,--)$ & $\varepsilon$ & \\
\hline 797 & 888 & $2^{3} \times 3 \times 37$ & $1 b$ & $5^{2} 2^{4} 3^{4} 37^{4}$ & 13 & 1 & 2 & 5 & 6 & $(\times,-,-,-)$ & $\gamma$ & 2,3 \\
\hline 798 & 889 & $7 \times 127$ & $1 b$ & $5^{2} 7^{4} 127^{4}$ & 4 & 0 & 0 & 1 & 6 & $(\times,-,-,-)$ & $\gamma$ & \\
\hline 799 & 890 & $2 \times 5 \times 89$ & 1a & $5^{6} 2^{4} 89^{4}$ & 16 & 1 & 1 & 3 & 4 & $(-, \otimes,-,--)$ & $\beta_{2}$ & $2^{2} \times 5, \mathcal{K}$ \\
\hline 800 & 891 & $3^{4} \times 11$ & $1 b$ & $5^{2} 3^{4} 11^{4}$ & 3 & 1 & 1 & 3 & 4 & $(-,-,(\times),-)$ & $\beta_{2}$ & $3 \times 5^{3}, \mathcal{K}$ \\
\hline 801 & 892 & $2^{2} \times 223$ & $1 b$ & $5^{2} 2^{4} 223^{4}$ & 3 & 0 & 0 & 1 & 6 & $(\times,-,-,-)$ & $\gamma$ & \\
\hline 802 & ${ }^{*} 893$ & $19 \times 47$ & 2 & $19^{4} 47^{4}$ & 1 & 1 & 1 & 3 & 4 & $(-, \otimes,-,-)$ & $\beta_{2}$ & $19, \mathfrak{L}$ \\
\hline 803 & *894 & $2 \times 3 \times 149$ & $1 b$ & $5^{2} 2^{4} 3^{4} 149^{4}$ & 12 & 1 & 2 & 5 & 6 & $(-, \times,-,-)$ & $\gamma$ & $5,2 \times 3^{2}$ \\
\hline 804 & 895 & $5 \times 179$ & 1a & $5^{6} 179^{4}$ & 4 & 1 & 1 & 2 & 3 & $(-, \otimes,-,-)$ & $\delta_{2}$ & $\mathcal{K}$ \\
\hline 805 & 897 & $3 \times 13 \times 23$ & $1 b$ & $5^{2} 3^{4} 13^{4} 23^{4}$ & 13 & 1 & 2 & 5 & 6 & $(\times,-,-,--)$ & $\gamma$ & $13 \times 5^{2}, 3^{3} \times 5^{2}$ \\
\hline 806 & 898 & $2 \times 449$ & $1 b$ & $5^{2} 2^{4} 449^{4}$ & 4 & 1 & 1 & 3 & 4 & $(-, \otimes,-,--)$ & $\beta_{2}$ & $2 \times 5^{2}, \mathcal{K}$ \\
\hline 807 & 899 & $29 \times 31$ & 2 & $29^{4} 31^{4}$ & 1 & 2 & 2 & 5 & 2 & $(-, \otimes,(\times),-)$ & $\alpha_{3}$ & $\mathcal{K}_{(29)}, \mathcal{K}_{(31)}$ \\
\hline 808 & 901 & $17 \times 53$ & 2 & $17^{4} 53^{4}$ & 1 & 0 & 0 & 0 & 5 & $(\times,-,-,-)$ & $\varepsilon$ & \\
\hline
\end{tabular}


Table 24. 47 pure metacyclic fields with normalized radicands $902 \leq D \leq 949$.

\begin{tabular}{|c|c|c|c|c|c|c|c|c|c|c|c|c|}
\hline No. & $D$ & Factors & $S$ & $f^{*}$ & $m$ & $V_{L}$ & $V_{M}$ & $V_{N}$ & $E$ & $(1,2,4,5)$ & $\mathrm{T}$ & $\mathrm{P}$ \\
\hline 809 & ${ }^{*} 902$ & $2 \times 11 \times 41$ & $1 b$ & $5^{2} 2^{4} 11^{4} 41^{4}$ & 13 & 2 & 3 & 7 & 4 & $(-,-,(\times),-)$ & $\beta_{2}$ & $11 \times 5, \mathcal{K}_{(11)} \times \mathcal{K}_{(41)}$ \\
\hline 810 & 903 & $3 \times 7 \times 43$ & $1 b$ & $5^{2} 3^{4} 7^{4} 43^{4}$ & 16 & 1 & 2 & 5 & 6 & $(\times,-,-,-)$ & $\gamma$ & $5,7^{2} \times 43$ \\
\hline 811 & 904 & $2^{3} \times 113$ & $1 \mathrm{~b}$ & $5^{2} 2^{4} 113^{4}$ & 3 & 0 & 0 & 1 & 6 & $(\times,-,-,--)$ & $\gamma$ & \\
\hline 812 & 905 & $5 \times 181$ & 1a & $5^{6} 181^{4}$ & 4 & 1 & 1 & 2 & 3 & $(-,-, \otimes,-)$ & $\alpha_{2}$ & $\mathcal{K}, \mathfrak{K}_{1}$ \\
\hline 813 & 906 & $2 \times 3 \times 151$ & $1 b$ & $5^{2} 2^{4} 3^{4} 151^{4}$ & 12 & 1 & 2 & 5 & 6 & $(-,-, \times,-)$ & $\gamma$ & $2,3 \times 5^{2}$ \\
\hline 814 & 907 & & 2 & $907^{4}$ & 1 & 0 & 0 & 0 & 5 & $(-,-,-, \otimes)$ & $\vartheta$ & \\
\hline 815 & 908 & $2^{2} \times 227$ & $1 \mathrm{~b}$ & $5^{2} 2^{4} 227^{4}$ & 3 & 0 & 0 & 1 & 6 & $(\times,-,-,--)$ & $\gamma$ & \\
\hline 816 & 909 & $3^{2} \times 101$ & $1 \mathrm{~b}$ & $5^{2} 3^{4} 101^{4}$ & 4 & 2 & 2 & 4 & 1 & $(-,-, \otimes,-)$ & $\alpha_{2}$ & $\mathcal{K}, \mathfrak{K}_{1}$ \\
\hline 817 & 910 & $2 \times 5 \times 7 \times 13$ & $1 \mathrm{a}$ & $5^{6} 2^{4} 7^{4} 13^{4}$ & 64 & 1 & 2 & 5 & 6 & $(\times,-,-,-)$ & $\gamma$ & $2,13 \times 5^{2}$ \\
\hline 818 & 911 & & $1 \mathrm{~b}$ & $5^{2} 911^{4}$ & 1 & 1 & 1 & 2 & 3 & $(-,-, \otimes,-)$ & $\alpha_{2}$ & $\mathcal{L}, \mathfrak{K}_{2}$ \\
\hline 819 & 912 & $2^{4} \times 3 \times 19$ & $1 \mathrm{~b}$ & $5^{2} 2^{4} 3^{4} 19^{4}$ & 13 & 1 & 2 & 5 & 6 & $(-, \times,-,-)$ & $\gamma$ & $3,2 \times 5^{2}$ \\
\hline 820 & 913 & $11 \times 83$ & $1 \mathrm{~b}$ & $5^{2} 11^{4} 83^{4}$ & 3 & 1 & 1 & 3 & 4 & $(-,-,(\times),-)$ & $\beta_{2}$ & $11 \times 5, \mathcal{K}$ \\
\hline 821 & 914 & $2 \times 457$ & $1 \mathrm{~b}$ & $5^{2} 2^{4} 457^{4}$ & 4 & 1 & 2 & 4 & 5 & $(\times,-,-,--)$ & $\varepsilon$ & 2 \\
\hline 822 & 915 & $3 \times 5 \times 61$ & 1a & $5^{6} 3^{4} 61^{4}$ & 16 & 1 & 1 & 3 & 4 & $(-,-,(\times),-)$ & $\beta_{2}$ & $3 \times 5^{2}, \mathcal{K}$ \\
\hline 823 & 916 & $2^{2} \times 229$ & $1 \mathrm{~b}$ & $5^{2} 2^{4} 229^{4}$ & 3 & 1 & 1 & 3 & 4 & $(-, \otimes,-,-)$ & $\beta_{2}$ & $2^{4} \times 5, \mathcal{K}$ \\
\hline 824 & 917 & $7 \times 131$ & $1 \mathrm{~b}$ & $5^{2} 7^{4} 131^{4}$ & 4 & 1 & 1 & 3 & 4 & $(-,-,(\times),-)$ & $\beta_{2}$ & $131 \times 5, \mathcal{K}$ \\
\hline 825 & 918 & $2 \times 3^{3} \times 17$ & 2 & $2^{4} 3^{4} 17^{4}$ & 3 & 0 & 0 & 1 & 6 & $(\times,-,-,-)$ & $\gamma$ & \\
\hline 826 & 919 & & $1 \mathrm{~b}$ & $5^{2} 919^{4}$ & 1 & 1 & 1 & 2 & 3 & $(-, \otimes,-,--)$ & $\delta_{2}$ & $\mathcal{L}$ \\
\hline 827 & 920 & $2^{3} \times 5 \times 23$ & la & $5^{6} 2^{4} 23^{4}$ & 16 & 0 & 0 & 1 & 6 & $(\times,-,-,-)$ & $\gamma$ & \\
\hline 828 & 921 & $3 \times 307$ & $1 \mathrm{~b}$ & $5^{2} 3^{4} 307^{4}$ & 4 & 0 & 0 & 1 & 6 & $(\times,-,-,-)$ & $\gamma$ & \\
\hline 829 & 922 & $2 \times 461$ & $1 \mathrm{~b}$ & $5^{2} 2^{4} 461^{4}$ & 3 & 1 & 2 & 4 & 5 & $(-,-, \times,-)$ & $\varepsilon$ & $2^{2} \times 5$ \\
\hline 830 & 923 & $13 \times 71$ & $1 \mathrm{~b}$ & $5^{2} 13^{4} 71^{4}$ & 3 & 2 & 2 & 4 & 1 & $(-,-, \otimes,-)$ & $\alpha_{2}$ & $\mathcal{K}, \mathfrak{K}_{2}$ \\
\hline 831 & ${ }^{*} 924$ & $2^{2} \times 3 \times 7 \times 11$ & 2 & $2^{4} 3^{4} 7^{4} 11^{4}$ & 12 & 1 & 2 & 5 & 6 & $(-,-, \times,-)$ & $\gamma$ & $3 \times 7,7 \times 11$ \\
\hline 832 & 925 & $5^{2} \times 37$ & 1a & $5^{6} 37^{4}$ & 4 & 0 & 0 & 0 & 5 & $(\times,-,-,-)$ & $\varepsilon$ & \\
\hline 833 & 926 & $2 \times 463$ & 2 & $2^{4} 463^{4}$ & 1 & 0 & 0 & 0 & 5 & $(\times,-,-,-)$ & $\varepsilon$ & \\
\hline 834 & 927 & $3^{2} \times 103$ & $1 \mathrm{~b}$ & $5^{2} 3^{4} 103^{4}$ & 3 & 1 & 2 & 4 & 5 & $(\times,-,-,-)$ & $\varepsilon$ & $3^{3} \times 5^{2}$ \\
\hline 835 & 929 & & $1 \mathrm{~b}$ & $5^{2} 929^{4}$ & 1 & 1 & 1 & 2 & 3 & $(-, \otimes,-,-)$ & $\delta_{2}$ & $\mathcal{L}$ \\
\hline 836 & 930 & $2 \times 3 \times 5 \times 31$ & $1 \mathrm{a}$ & $5^{6} 2^{4} 3^{4} 31^{4}$ & 64 & 2 & 4 & 9 & 6 & $(-,-, \times,-)$ & $\gamma$ & 5,31 \\
\hline 837 & 931 & $7^{2} \times 19$ & $1 \mathrm{~b}$ & $5^{2} 7^{4} 19^{4}$ & 4 & 1 & 1 & 3 & 4 & $(-, \otimes,-,-)$ & $\beta_{2}$ & $7, \mathcal{L}$ \\
\hline 838 & 932 & $2^{2} \times 233$ & 2 & $2^{4} 233^{4}$ & 1 & 0 & 0 & 0 & 5 & $(\times,-,-,--)$ & $\varepsilon$ & \\
\hline 839 & 933 & $3 \times 311$ & $1 \mathrm{~b}$ & $5^{2} 3^{4} 311^{4}$ & 3 & 1 & 1 & 3 & 4 & $(-,-,(\times),-)$ & $\beta_{2}$ & $3 \times 5, \mathcal{K}$ \\
\hline 840 & 934 & $2 \times 467$ & $1 \mathrm{~b}$ & $5^{2} 2^{4} 467^{4}$ & 3 & 0 & 0 & 1 & 6 & $(\times,-,-,-)$ & $\gamma$ & \\
\hline 841 & 935 & $5 \times 11 \times 17$ & $1 \mathrm{a}$ & $5^{6} 11^{4} 17^{4}$ & 16 & 2 & 2 & 4 & 1 & $(-,-, \otimes,-)$ & $\alpha_{2}$ & $\mathcal{K}, \mathfrak{K}_{2}$ \\
\hline 842 & 936 & $2^{3} \times 3^{2} \times 13$ & $1 \mathrm{~b}$ & $5^{2} 2^{4} 3^{4} 13^{4}$ & 13 & 1 & 2 & 5 & 6 & $(\times,-,-,-)$ & $\gamma$ & 3,5 \\
\hline 843 & 937 & & $1 \mathrm{~b}$ & $5^{2} 937^{4}$ & 1 & 0 & 0 & 0 & 5 & $(\times,-,-,-)$ & $\varepsilon$ & \\
\hline 844 & 938 & $2 \times 7 \times 67$ & $1 \mathrm{~b}$ & $5^{2} 2^{4} 7^{4} 67^{4}$ & 12 & 1 & 2 & 5 & 6 & $(\times,-,-,-)$ & $\gamma$ & $7,67 \times 5$ \\
\hline
\end{tabular}




\section{Continued}

\begin{tabular}{ccccccccccccc}
\hline 845 & 939 & $3 \times 313$ & $1 \mathrm{~b}$ & $5^{2} 3^{4} 313^{4}$ & 3 & 1 & 2 & 4 & 5 & $(\times,-,-,-)$ & $\varepsilon$ & 5 \\
846 & 940 & $2^{2} \times 5 \times 47$ & $1 \mathrm{a}$ & $5^{6} 2^{4} 47^{4}$ & 16 & 0 & 0 & 1 & 6 & $(\times,-,-,-)$ & $\gamma$ & \\
847 & 941 & & $\mathrm{lb}$ & $5^{2} 941^{4}$ & 1 & 2 & 2 & 4 & 1 & $(-,-, \otimes,-)$ & $\alpha_{2}$ & $\mathcal{L}, \mathfrak{K}_{1}$ \\
848 & 942 & $2 \times 3 \times 157$ & $1 \mathrm{~b}$ & $5^{2} 2^{4} 3^{4} 157^{4}$ & 12 & 2 & 4 & 8 & 5 & $(\times,-,-,-)$ & $\varepsilon$ & 157 \\
849 & 943 & $23 \times 41$ & 2 & $23^{4} 41^{4}$ & 1 & 1 & 1 & 2 & 3 & $(-,-, \otimes,-)$ & $\alpha_{2}$ & $\mathcal{K}, \mathfrak{K}_{2}$ \\
850 & 944 & $2^{4} \times 59$ & $1 \mathrm{~b}$ & $5^{2} 2^{4} 59^{4}$ & 3 & 1 & 1 & 3 & 4 & $(-, \otimes,-,-)$ & $\beta_{2}$ & $2 \times 5^{4}, \mathcal{K}$ \\
851 & 945 & $3^{3} \times 5 \times 7$ & $1 \mathrm{a}$ & $5^{6} 3^{4} 7^{4}$ & 16 & 0 & 0 & 1 & 6 & $(\times,-,-,-)$ & $\gamma$ & \\
852 & 946 & $2 \times 11 \times 43$ & $1 \mathrm{~b}$ & $5^{2} 2^{4} 11^{4} 43^{4}$ & 12 & 1 & 2 & 5 & 6 & $(-,-, \times,-)$ & $\gamma$ & $2 \times 5,43$ \\
853 & 947 & & $1 \mathrm{~b}$ & $5^{2} 947^{4}$ & 1 & 0 & 0 & 0 & 5 & $(\times,-,-,-)$ & $\varepsilon$ & \\
854 & 948 & $2^{2} \times 3 \times 79$ & $1 \mathrm{~b}$ & $5^{2} 2^{4} 3^{4} 79^{4}$ & 13 & 1 & 2 & 5 & 6 & $(-, \times,-,-)$ & $\gamma$ & $2 \times 5^{2}, 3 \times 5^{2}$ \\
855 & 949 & $13 \times 73$ & 2 & $13^{4} 73^{4}$ & 1 & 0 & 0 & 0 & 5 & $(\times,-,-,-)$ & $\varepsilon$ & \\
\hline
\end{tabular}

Table 25. 45 pure metacyclic fields with normalized radicands $950 \leq D \leq 999$.

\begin{tabular}{|c|c|c|c|c|c|c|c|c|c|c|c|c|}
\hline No. & $D$ & Factors & $S$ & $f^{\prime}$ & $m$ & $V_{L}$ & $V_{M}$ & $V_{N}$ & $E$ & $(1,2,4,5)$ & $\mathrm{T}$ & $\mathrm{P}$ \\
\hline 856 & 950 & $2 \times 5^{2} \times 19$ & $1 \mathrm{a}$ & $5^{6} 2^{4} 19^{4}$ & 16 & 1 & 1 & 3 & 4 & $(-, \otimes,-,-)$ & $\beta_{2}$ & $5, \mathcal{K}$ \\
\hline 857 & 951 & $3 \times 317$ & 2 & $3^{4} 317^{4}$ & 1 & 0 & 0 & 0 & 5 & $(\times,-,-,-)$ & $\varepsilon$ & \\
\hline 858 & 952 & $2^{3} \times 7 \times 17$ & $1 b$ & $5^{2} 2^{4} 7^{4} 17^{4}$ & 12 & 1 & 2 & 5 & 6 & $(\times,-,-,--)$ & $\gamma$ & $2 \times 5^{4}, 7 \times 5^{4}$ \\
\hline 859 & 953 & & $1 b$ & $5^{2} 953^{4}$ & 1 & 0 & 0 & 0 & 5 & $(\times,-,-,-)$ & $\varepsilon$ & \\
\hline 860 & 954 & $2 \times 3^{2} \times 53$ & $1 b$ & $5^{2} 2^{4} 3^{4} 53^{4}$ & 13 & 1 & 2 & 5 & 6 & $(\times,-,-,-)$ & $\gamma$ & $3 \times 5,2 \times 5^{2}$ \\
\hline 861 & $* 955$ & $5 \times 191$ & 1a & $5^{6} 191^{4}$ & 4 & $2^{*}$ & $3^{*}$ & $6^{*}$ & 3 & $(-,-, \otimes,-)$ & $\alpha_{2}$ & $\mathcal{K}, \mathfrak{K}_{1}$ \\
\hline 862 & 956 & $2^{2} \times 239$ & $1 \mathrm{~b}$ & $5^{2} 2^{4} 239^{4}$ & 3 & 1 & 1 & 3 & 4 & $(-, \otimes,-,-)$ & $\beta_{2}$ & $239, \mathcal{L}$ \\
\hline 863 & $* 957$ & $3 \times 11 \times 29$ & 2 & $3^{4} 11^{4} 29^{4}$ & 3 & 2 & 2 & 5 & 2 & $(-, \otimes,(\times),-)$ & $\alpha_{3}$ & $\mathcal{K}_{(11)}, \mathcal{K}_{(29)}$ \\
\hline 864 & 958 & $2 \times 479$ & $1 \mathrm{~b}$ & $5^{2} 2^{4} 479^{4}$ & 3 & 1 & 2 & 4 & 5 & $(-, \times,-,-)$ & $\varepsilon$ & $2^{3} \times 5$ \\
\hline 865 & 959 & $7 \times 137$ & $1 \mathrm{~b}$ & $5^{2} 7^{4} 137^{4}$ & 4 & 0 & 0 & 1 & 6 & $(\times,-,-,-)$ & $\gamma$ & \\
\hline 866 & 962 & $2 \times 13 \times 37$ & $1 b$ & $5^{2} 2^{4} 13^{4} 37^{4}$ & 13 & 1 & 2 & 5 & 6 & $(\times,-,-,-)$ & $\gamma$ & $2 \times 5,37 \times 5$ \\
\hline 867 & 963 & $3^{2} \times 107$ & $1 b$ & $5^{2} 3^{4} 107^{4}$ & 4 & 0 & 0 & 1 & 6 & $(\times,-,-,-)$ & $\gamma$ & \\
\hline 868 & 964 & $2^{2} \times 241$ & $1 \mathrm{~b}$ & $5^{2} 2^{4} 241^{4}$ & 3 & 1 & 2 & 4 & 5 & $(-,-, \otimes,-)$ & $\beta_{1}$ & $241, \mathfrak{K}_{2}$ \\
\hline 869 & 965 & $5 \times 193$ & 1a & $5^{6} 193^{4}$ & 4 & 0 & 0 & 1 & 6 & $(-,-,-, \otimes)$ & $\eta$ & \\
\hline 870 & 966 & $2 \times 3 \times 7 \times 23$ & $1 \mathrm{~b}$ & $5^{2} 2^{4} 3^{4} 7^{4} 23^{4}$ & 52 & 2 & 4 & 9 & 6 & $(\times,-,-,-)$ & $\gamma$ & $3 \times 5^{3}, 2 \times 23^{2}$ \\
\hline 871 & 967 & & $1 b$ & $5^{2} 967^{4}$ & 1 & 0 & 0 & 0 & 5 & $(\times,-,-,-)$ & $\varepsilon$ & \\
\hline 872 & 969 & $3 \times 17 \times 19$ & $1 b$ & $5^{2} 3^{4} 17^{4} 19^{4}$ & 13 & 1 & 2 & 5 & 6 & $(-, \times,-,-)$ & $\gamma$ & $19,3 \times 5^{2}$ \\
\hline 873 & 970 & $2 \times 5 \times 97$ & 1a & $5^{6} 2^{4} 97^{4}$ & 16 & 0 & 0 & 1 & 6 & $(\times,-,-,-)$ & $\gamma$ & \\
\hline 874 & 971 & & $1 b$ & $5^{2} 971^{4}$ & 1 & 2 & 2 & 4 & 1 & $(-,-, \otimes,-)$ & $\alpha_{2}$ & $\mathcal{L}, \mathfrak{K}_{1}$ \\
\hline 875 & 973 & $7 \times 139$ & $1 \mathrm{~b}$ & $5^{2} 7^{4} 139^{4}$ & 4 & 1 & 1 & 3 & 4 & $(-, \otimes,-,-)$ & $\beta_{2}$ & $7 \times 5^{2}, \mathcal{K}$ \\
\hline 876 & 974 & $2 \times 487$ & 2 & $2^{4} 487^{4}$ & 1 & 0 & 0 & 0 & 5 & $(\times,-,-,-)$ & $\varepsilon$ & \\
\hline 877 & 975 & $3 \times 5^{2} \times 13$ & 1a & $5^{6} 3^{4} 13^{4}$ & 16 & 0 & 0 & 1 & 6 & $(\times,-,-,-)$ & $\gamma$ & \\
\hline 878 & 976 & $2^{4} \times 61$ & 2 & $2^{4} 61^{4}$ & 1 & 1 & 1 & 2 & 3 & $(-,-, \otimes,-)$ & $\alpha_{2}$ & $\mathcal{K}, \mathfrak{K}_{2}$ \\
\hline
\end{tabular}




\section{Continued}

\begin{tabular}{|c|c|c|c|c|c|c|c|c|c|c|c|c|}
\hline 879 & 977 & & $1 \mathrm{~b}$ & $5^{2} 977^{4}$ & 1 & 0 & 0 & 0 & 5 & $(\times,-,-,-)$ & $\varepsilon$ & \\
\hline 880 & 978 & $2 \times 3 \times 163$ & $1 \mathrm{~b}$ & $5^{2} 2^{4} 3^{4} 163^{4}$ & 13 & 1 & 2 & 5 & 6 & $(\times,-,-,-)$ & $\gamma$ & $3 \times 5^{2}, 2 \times 5^{4}$ \\
\hline 881 & 979 & $11 \times 89$ & $1 \mathrm{~b}$ & $5^{2} 11^{4} 89^{4}$ & 3 & 2 & 3 & 7 & 4 & $(-, \otimes,(\times),-)$ & $\beta_{2}$ & $11, \mathcal{K}_{(11)} \cdot \mathcal{K}_{(89}^{2}$ \\
\hline 882 & 980 & $2^{2} \times 5 \times 7^{2}$ & la & $5^{6} 2^{4} 7^{4}$ & 16 & 1 & 2 & 4 & 5 & $(\times,-,-,--)$ & $\varepsilon$ & 7 \\
\hline 883 & 981 & $3^{2} \times 109$ & $1 \mathrm{~b}$ & $5^{2} 3^{4} 109^{4}$ & 3 & 1 & 1 & 3 & 4 & $(-, \otimes,-,-)$ & $\beta_{2}$ & $109, \mathcal{L}$ \\
\hline 884 & *982 & $2 \times 491$ & 2 & $2^{4} 491^{4}$ & 1 & 1 & 1 & 2 & 3 & $(-,-, \otimes,-)$ & $\alpha_{2}$ & $\mathcal{K}, \mathfrak{K}_{1}$ \\
\hline 885 & 983 & & $1 \mathrm{~b}$ & $5^{2} 983^{4}$ & 1 & 0 & 0 & 0 & 5 & $(\times,-,-,-)$ & $\varepsilon$ & \\
\hline 886 & 984 & $2^{3} \times 3 \times 41$ & $1 b$ & $5^{2} 2^{4} 3^{4} 41^{4}$ & 13 & 1 & 2 & 5 & 6 & $(-,-, \times,-)$ & $\gamma$ & $3,2 \times 5^{2}$ \\
\hline 887 & 985 & $5 \times 197$ & 1a & $5^{6} 197^{4}$ & 4 & 0 & 0 & 0 & 5 & $(\times,-,-,-)$ & $\varepsilon$ & \\
\hline 888 & 986 & $2 \times 17 \times 29$ & $1 \mathrm{~b}$ & $5^{2} 2^{4} 17^{4} 29^{4}$ & 13 & 2 & 4 & 8 & 5 & $(-, \times,-,-)$ & $\varepsilon$ & $2 \times 5$ \\
\hline 889 & 987 & $3 \times 7 \times 47$ & $1 \mathrm{~b}$ & $5^{2} 3^{4} 7^{4} 47^{4}$ & 12 & 1 & 2 & 5 & 6 & $(\times,-,-,-)$ & $\gamma$ & $7 \times 5^{2}, 3 \times 5^{4}$ \\
\hline 890 & 988 & $2^{2} \times 13 \times 19$ & $1 \mathrm{~b}$ & $5^{2} 2^{4} 13^{4} 19^{4}$ & 13 & 1 & 2 & 5 & 6 & $(-, \times,-,-)$ & $\gamma$ & $2,13 \times 5^{2}$ \\
\hline 891 & 989 & $23 \times 43$ & $1 \mathrm{~b}$ & $5^{2} 23^{4} 43^{4}$ & 4 & 0 & 0 & 1 & 6 & $(\times,-,-,-)$ & $\gamma$ & \\
\hline 892 & 990 & $2 \times 3^{2} \times 5 \times 11$ & $1 \mathrm{a}$ & $5^{6} 2^{4} 3^{4} 11^{4}$ & 64 & 1 & 2 & 5 & 6 & $(-,-, \times,-)$ & $\gamma$ & $2 \times 5,3 \times 5^{2}$ \\
\hline 893 & 991 & & $1 b$ & $5^{2} 991^{4}$ & 1 & 1 & 2 & 3 & 4 & $(-,-, \otimes,-)$ & $\delta_{1}$ & $\mathfrak{K}_{1}$ \\
\hline 894 & 993 & $3 \times 331$ & 2 & $3^{4} 331^{4}$ & 1 & 1 & 1 & 2 & 3 & $(-,-, \otimes,-)$ & $\alpha_{2}$ & $\mathcal{K}, \mathfrak{K}_{2}$ \\
\hline 895 & 994 & $2 \times 7 \times 71$ & $1 \mathrm{~b}$ & $5^{2} 2^{4} 7^{4} 71^{4}$ & 12 & 2 & 3 & 7 & 4 & $(-,-,(\times),-)$ & $\beta_{2}$ & $2 \times 5^{3}, \mathcal{K}$ \\
\hline 896 & 995 & $5 \times 199$ & $1 \mathrm{a}$ & $5^{6} 199^{4}$ & 4 & 1 & 1 & 3 & 4 & $(-, \otimes,-, \otimes)$ & $\zeta_{2}$ & $\mathcal{K}$ \\
\hline 897 & 996 & $2^{2} \times 3 \times 83$ & $1 \mathrm{~b}$ & $5^{2} 2^{4} 3^{4} 83^{4}$ & 13 & 1 & 2 & 5 & 6 & $(\times,-,-,-)$ & $\gamma$ & $2 \times 5^{2}, 3 \times 5^{4}$ \\
\hline 898 & 997 & & $1 b$ & $5^{2} 997^{4}$ & 1 & 0 & 0 & 0 & 5 & $(\times,-,-,-)$ & $\varepsilon$ & \\
\hline 899 & 998 & $2 \times 499$ & $1 \mathrm{~b}$ & $5^{2} 2^{4} 499^{4}$ & 4 & 1 & 1 & 3 & 4 & $(-, \otimes,-,-)$ & $\beta_{2}$ & $499, \mathcal{L}$ \\
\hline 900 & 999 & $3^{3} \times 37$ & 2 & $3^{4} 37^{4}$ & 1 & 0 & 0 & 0 & 5 & $(\times,-,-,-)$ & $\varepsilon$ & \\
\hline
\end{tabular}

Table 26. Absolute frequencies of differential principal factorization types.

\begin{tabular}{cccccccccccc}
\hline Type & 100 & 200 & 300 & 400 & 500 & 600 & 700 & 800 & 900 & 1000 & $\%$ \\
\hline$\alpha_{1}$ & 1 & 2 & 3 & 4 & 5 & 5 & 5 & 9 & 9 & 9 & \\
$\alpha_{2}$ & 10 & 17 & 23 & 30 & 35 & 42 & 52 & 57 & 63 & 75 & 8.3 \\
$\alpha_{3}$ & 0 & 0 & 0 & 1 & 1 & 3 & 5 & 5 & 7 & 8 & \\
$\beta_{1}$ & 0 & 2 & 4 & 7 & 8 & 11 & 15 & 18 & 22 & 23 & \\
$\beta_{2}$ & 7 & 24 & 40 & 54 & 80 & 94 & 108 & 126 & 146 & 161 & 17.9 \\
$\gamma$ & 25 & 55 & 88 & 117 & 148 & 187 & 222 & 259 & 290 & 324 & 36.0 \\
$\delta_{1}$ & 0 & 0 & 1 & 1 & 3 & 4 & 4 & 4 & 6 & 7 & \\
$\delta_{2}$ & 8 & 14 & 19 & 23 & 31 & 35 & 38 & 44 & 51 & 53 & 5.9 \\
$\varepsilon$ & 26 & 45 & 67 & 95 & 110 & 128 & 150 & 165 & 184 & 208 & 23.4 \\
$\zeta_{1}$ & 0 & 1 & 1 & 1 & 1 & 1 & 1 & 1 & 1 & 1 & \\
$\zeta_{2}$ & 0 & 0 & 0 & 0 & 0 & 1 & 1 & 4 & 4 & 5 & \\
$\eta$ & 1 & 2 & 4 & 5 & 5 & 6 & 6 & 6 & 6 & 7 & \\
$\vartheta$ & 3 & 6 & 8 & 9 & 11 & 13 & 15 & 17 & 18 & 19 & \\
Total & 81 & 168 & 258 & 347 & 438 & 530 & 622 & 715 & 807 & 900 & 100.0 \\
\hline
\end{tabular}


It is striking that type $\alpha_{1}$ with 2 -dimensional relative principal factorization, $R=2$, and type $\alpha_{3}$ with 2 -dimensional intermediate principal factorization, $I=2$, are populated rather sparsely, in favour of a remarkable contribution by type $\alpha_{2}$ with mixed intermediate and relative principal factorization, $I=R=1 \quad$ (place four with $8 \%$ ).

The appearance of the four types $\zeta_{1}, \zeta_{2}, \eta, \vartheta$ with norm representation $N_{N / K}(Z)=\zeta, Z \in U_{N}$, of the primitive fifth root of unity $\zeta=\zeta_{5}$ is marginal ([6] Thm. 8.2), in spite of the parametrized contribution by all prime conductors $f=q \equiv \pm 7 \bmod (25)$ to type $\vartheta$, as we shall prove in Theorem 4.1 (1) in $\S 4.3$.

\subsection{Similarity Classes and Prototypes}

In [7], we came to the conviction that for deeper insight into the arithmetical structure of the fields under investigation, the prime factorization of the class field theoretic conductor $f$ of the abelian extension $N / K$ over the cyclotomic field $K=\mathbb{Q}(\zeta)$ and the primary invariants of all involved 5-class groups must be taken in consideration. These ideas were inspired by [16] [17] and have lead to the concept of similarity classes and representative prototypes, which refines the differential principal factorization (DPF) types

Let $t$ be the number of primes $q_{1}, \cdots, q_{t} \in \mathbb{P}$ distinct from 5 which divide the conductor $f$. Among these prime numbers, we separately count $u:=\#\left\{1 \leq i \leq t \mid q_{i} \equiv \pm 1, \pm 7 \bmod (25)\right\}$ free primes, $v:=t-u$ restrictive primes, $s_{2}:=\#\left\{1 \leq i \leq t \mid q_{i} \equiv-1 \bmod (5)\right\} \quad 2$-split primes, and $s_{4}:=\#\left\{1 \leq i \leq t \mid q_{i} \equiv+1 \bmod (5)\right\} \quad 4$-split primes. The multiplicity $m=m(f)$ is given in terms of $t, u, v$, according to $\S 2$, and the dimensions of various spaces of primitive ambiguous ideals over the finite field $\mathbb{F}_{5}$ are given in terms of $t, s_{2}, s_{4}$, according to [6] $\S 4$. By $\eta=\frac{1}{2}(1+\sqrt{5})$ we denote the fundamental unit of $K^{+}=\mathbb{Q}(\sqrt{5})$. The dimensions of the spaces of absolute, intermediate and relative DPF over $\mathbb{F}_{5}$ are denoted by $A, I$ and $R$, identical with the additive (logarithmic) version in ([6] Thm. 6.1). Further, let $M=\mathbb{Q}(\sqrt{5}, \sqrt[5]{D})$ be the maximal real intermediate field of $N / L$, and denote by $U_{0}$ the subgroup of the unit group $U_{N}$ of $N=\mathbb{Q}(\zeta, \sqrt[5]{D})$ generated by the units of all conjugate fields of $L=\mathbb{Q}(\sqrt[5]{D})$ and of $K=\mathbb{Q}(\zeta)$, where $\zeta=\zeta_{5}$ is a primitive fifth root of unity. For a number field $F$, let $V_{F}:=v_{5}(\# \mathrm{Cl}(F))$ be the 5 -valuation of the class number of $F$.

Definition 4.1 A set of normalized fifth power free radicands $D>1$ is called a similarity class if the associated pure quintic fields $L=\mathbb{Q}(\sqrt[5]{D})$ share the following common multiplets of invariants:

- the refined Dedekind species $\left(e_{0} ; t, u, v, m ; n, s_{2}, s_{4}\right)$, where

$$
f^{4}=5^{e_{0}} \cdot q_{1}^{4} \cdots q_{t}^{4} \text { with } e_{0} \in\{0,2,6\}, t \geq 0, n=t-s_{2}-s_{4},
$$

- the differential principal factorization type $(U, \eta, \zeta ; A, I, R)$, where

$$
\left(U_{K}: N_{N / K}\left(U_{N}\right)\right)=5^{U} \text { and } U+1=A+I+R \text {, }
$$


- the structure of the 5-class groups $\left(V_{L}, V_{M}, V_{N} ; E\right)$, where

$$
\left(U_{N}: U_{0}\right)=5^{E} \text { and } V_{N}=4 \cdot V_{L}+E-5 \text {. }
$$

Warning 4.1 To reduce the number of invariants, we abstain from defining additional counters $s_{2}^{\prime}:=\#\left\{1 \leq i \leq t \mid q_{i} \equiv-1 \bmod (25)\right\}$ and $s_{4}^{\prime}:=\#\left\{1 \leq i \leq t \mid q_{i} \equiv+1 \bmod (25)\right\} \quad$ for free splitting prime divisors of the conductor $\mathrm{f}$. However, we point out that occasionally a similarity class in the sense of Definition 4.1 will be split in two separate classes, having the same invariants, but distinct contributions to the counters $\mathrm{u}$ and $s_{4}$, resp. $s_{2}$. For instance, the similarity classes [77] and [202] with $77=7 \times 11$ and $202=2 \times 101$ share identical multiplets of invariants $\left(e_{0} ; t, u, v, m ; n, s_{2}, s_{4}\right)=(2 ; 2,1,1,4 ; 1,0,1) \quad($ species $1 \mathrm{~b})$, $(U, \eta, \zeta ; A, I, R)=(2,-,-; 2,1,0)$ (type $\left.\beta_{2}\right)$, and $\left(V_{L}, V_{M}, V_{N} ; E\right)=(1,1,3 ; 4)$. But $u=1$ and $n=1$ are due to $7, v=1$ and $s_{4}=1$ are due to 11 , in the former case, whereas $v=1$ and $n=1$ are due to $2, u=1$ and $s_{4}=1$ are due to 101 , in the latter case. Therefore, the contributions by primes congruent to $\pm 1 \bmod (25)$ will be indicated by writing $u=1^{\prime}$ and $s_{4}=1^{\prime}$, resp. $s_{2}=1^{\prime}$.

We also emphasize that in the rare cases of non-elementary 5-class groups, the actual structures (abelian type invariants) of the 5-class groups will be taken into account, and not only the 5-valuations $V_{L}, V_{M}, V_{N}$.

Definition 4.2 The minimal element $\mathrm{M}$ of a similarity class (with respect to the natural order of positive integers $\mathbb{N}$ ) is called the representative prototype of the class, which is denoted by writing its prototype in square brackets $[\mathbf{M}]$.

The remaining elements of a similarity class, which are bigger than the prototype, only reproduce the arithmetical invariants of the prototype and do not provide any additional information, exept possibly about other primary components of the class groups, that is the structure of $\ell$-class groups $\mathrm{Cl}_{\ell}(F)$ of the fields $F \in\{L, M, N\}$ for $\ell \in \mathbb{P} \backslash\{5\}$.

Whereas there are only 13 DPF types of pure quintic fields, the number of similarity classes is obviously infinite, since firstly the number $t$ of primes dividing the conductor is unbounded and secondly the number of states, defined by the triplet $\left(V_{L}, V_{M}, V_{N}\right)$ of 5-valuations of class numbers, is also unlimited.

Given a fixed refined Dedekind species $\left(e_{0} ; t, u, v, m ; n, s_{2}, s_{4}\right)$, the set of all associated normalized fifth power free radicands $D$ usually splits into several similarity classes defined by distinct DPF types (type splitting). Occasionally it even splits further into different structures of 5-class groups, called states, with increasing complexity of abelian type invariants (state splitting).

The 134 prototypes $2 \leq \mathbf{M}<10^{3}$ of pure quintic fields are listed in the Tables 27-30. By $|\mathbf{M}|:=\#\{D \in[\mathbf{M}] \mid D<B\}$ we denote the number of elements of the similarity class $[\mathbf{M}]$ defined by the prototype $\mathbf{M}$, truncated at the upper bound $B:=10^{3}$ of our systematic investigations.

\subsection{General Theorems on DPF Types and Polya Fields}

There is only a single finite similarity class $[5]=\{5\}$, characterized by the 
Table 27. 46 prototypes $2 \leq \mathbf{M}<200$ of pure metacyclic fields.

\begin{tabular}{|c|c|c|c|c|c|c|c|c|c|c|c|c|c|}
\hline No. & M & Factors & S & $f^{4}$ & $m$ & $V_{L}$ & $V_{M}$ & $V_{N}$ & $E$ & $(1,2,4,5)$ & $\mathrm{T}$ & $\mathrm{P}$ & $|\mathbf{M}|$ \\
\hline 1 & 2 & & $1 b$ & $5^{2} 2^{4}$ & 1 & 0 & 0 & 0 & 5 & $(\times,-,-,-)$ & $\varepsilon$ & & 71 \\
\hline 2 & 5 & & $1 \mathrm{a}$ & $5^{6}$ & 1 & 0 & 0 & 0 & 5 & $(-,-,-, \otimes)$ & $\vartheta$ & & 1 \\
\hline 3 & 6 & $2 \times 3$ & $1 \mathrm{~b}$ & $5^{2} 2^{4} 3^{4}$ & 3 & 0 & 0 & 1 & 6 & $(\times,-,-,--)$ & $\gamma$ & & 77 \\
\hline 4 & 7 & & 2 & $7^{4}$ & 1 & 0 & 0 & 0 & 5 & $(-,-,-, \otimes)$ & $\vartheta$ & & 18 \\
\hline 5 & 10 & $2 \times 5$ & 1a & $5^{6} 2^{4}$ & 4 & 0 & 0 & 0 & 5 & $(\times,-,-,-)$ & $\varepsilon$ & & 31 \\
\hline 6 & 11 & & $1 \mathrm{~b}$ & $5^{2} 11^{4}$ & 1 & 1 & 1 & 2 & 3 & $(-,-, \otimes,-)$ & $\alpha_{2}$ & $\mathcal{L}, \mathfrak{K}_{1}$ & 14 \\
\hline 7 & 14 & $2 \times 7$ & $1 \mathrm{~b}$ & $5^{2} 2^{4} 7^{4}$ & 4 & 0 & 0 & 1 & 6 & $(\times,-,-,-)$ & $\gamma$ & & 44 \\
\hline 8 & 18 & $2 \times 3^{2}$ & 2 & $2^{4} 3^{4}$ & 1 & 0 & 0 & 0 & 5 & $(\times,-,-,-)$ & $\varepsilon$ & & 37 \\
\hline 9 & 19 & & $1 \mathrm{~b}$ & $5^{2} 19^{4}$ & 1 & 1 & 1 & 2 & 3 & $(-, \otimes,-,-)$ & $\delta_{2}$ & $\mathcal{L}$ & 27 \\
\hline 10 & 22 & $2 \times 11$ & $1 \mathrm{~b}$ & $5^{2} 2^{4} 11^{4}$ & 3 & 1 & 1 & 3 & 4 & $(-,-,(\times),-)$ & $\beta_{2}$ & $2 \times 5, \mathcal{K}$ & 35 \\
\hline 11 & 30 & $2 \times 3 \times 5$ & 1a & $5^{6} 2^{4} 3^{4}$ & 16 & 0 & 0 & 1 & 6 & $(\times,-,-,-)$ & $\gamma$ & & 37 \\
\hline 12 & 31 & & $1 \mathrm{~b}$ & $5^{2} 31^{4}$ & 1 & 2 & 3 & 5 & 2 & $(-,-, \otimes,-)$ & $\alpha_{1}$ & $\mathfrak{K}_{1}, \mathfrak{K}_{2}$ & 2 \\
\hline 13 & 33 & $3 \times 11$ & $1 b$ & $5^{2} 3^{4} 11^{4}$ & 3 & 2 & 2 & 4 & 1 & $(-,-, \otimes,-)$ & $\alpha_{2}$ & $\mathcal{K}, \mathfrak{K}_{2}$ & 8 \\
\hline 14 & 35 & $5 \times 7$ & $1 \mathrm{a}$ & $5^{6} 7^{4}$ & 4 & 0 & 0 & 1 & 6 & $(-,-,-, \otimes)$ & $\eta$ & & 6 \\
\hline 15 & 38 & $2 \times 19$ & $1 \mathrm{~b}$ & $5^{2} 2^{4} 19^{4}$ & 3 & 1 & 1 & 3 & 4 & $(-, \otimes,-,-)$ & $\beta_{2}$ & $5, \mathcal{K}$ & 44 \\
\hline 16 & 42 & $2 \times 3 \times 7$ & $1 \mathrm{~b}$ & $5^{2} 2^{4} 3^{4} 7^{4}$ & 12 & 1 & 2 & 5 & 6 & $(\times,-,-,-)$ & $\gamma$ & $2 \times 5,3 \times 5^{2}$ & 22 \\
\hline 17 & 55 & $5 \times 11$ & $1 \mathrm{a}$ & $5^{6} 11^{4}$ & 4 & 1 & 1 & 2 & 3 & $(-,-, \otimes,-)$ & $\alpha_{2}$ & $\mathcal{K}, \mathfrak{K}_{1}$ & 6 \\
\hline 18 & 57 & $3 \times 19$ & 2 & $3^{4} 19^{4}$ & 1 & 1 & 1 & 2 & 3 & $(-, \otimes,-,-)$ & $\delta_{2}$ & $\mathcal{K}$ & 10 \\
\hline 19 & 66 & $2 \times 3 \times 11$ & $1 \mathrm{~b}$ & $5^{2} 2^{4} 3^{4} 11^{4}$ & 13 & 1 & 2 & 5 & 6 & $(-,-, \times,-)$ & $\gamma$ & $2 \times 5,3 \times 5^{3}$ & 17 \\
\hline 20 & 70 & $2 \times 5 \times 7$ & $1 \mathrm{a}$ & $5^{6} 2^{4} 7^{4}$ & 16 & 0 & 0 & 1 & 6 & $(\times,-,-,-)$, & $\gamma$ & & 14 \\
\hline 21 & 77 & $7 \times 11$ & $1 \mathrm{~b}$ & $5^{2} 7^{4} 11^{4}$ & 4 & 1 & 1 & 3 & 4 & $(-,-,(\times),-)$ & $\beta_{2}$ & $11 \times 5^{3}, \mathcal{K}$ & 7 \\
\hline 22 & 78 & $2 \times 3 \times 13$ & $1 \mathrm{~b}$ & $5^{2} 2^{4} 3^{4} 13^{4}$ & 13 & 1 & 2 & 5 & 6 & $(\times,-,-,--)$ & $\gamma$ & $3,2 \times 5^{3}$ & 37 \\
\hline 23 & 82 & $2 \times 41$ & 2 & $2^{4} 41^{4}$ & 1 & 1 & 1 & 2 & 3 & $(-,-, \otimes,-)$ & $\alpha_{2}$ & $\mathcal{K}, \mathfrak{K}_{2}$ & 15 \\
\hline 24 & 95 & $5 \times 19$ & 1a & $5^{6} 19^{4}$ & 4 & 1 & 1 & 2 & 3 & $(-, \otimes,-,-)$ & $\delta_{2}$ & $\mathcal{K}$ & 9 \\
\hline 25 & 101 & & 2 & $101^{4}$ & 1 & 1 & 2 & 4 & 5 & $(-,-, \otimes, \otimes)$ & $\zeta_{1}$ & $\mathfrak{K}_{2}$ & 1 \\
\hline 26 & 110 & $2 \times 5 \times 11$ & la & $5^{6} 2^{4} 11^{4}$ & 16 & 1 & 1 & 3 & 4 & $(-,-,(\times),-)$ & $\beta_{2}$ & $11, \mathcal{L}$ & 11 \\
\hline 27 & 114 & $2 \times 3 \times 19$ & $1 \mathrm{~b}$ & $5^{2} 2^{4} 3^{4} 19^{4}$ & 13 & 1 & 2 & 5 & 6 & $(-, \times,-,-)$ & $\gamma$ & $2 \times 5^{3}, 3 \times 5^{3}$ & 20 \\
\hline 28 & 123 & $3 \times 41$ & $1 \mathrm{~b}$ & $5^{2} 3^{4} 41^{4}$ & 3 & 2 & 3 & 6 & 3 & $(-,-, \otimes,-)$ & $\alpha_{2}$ & $\mathcal{K}, \mathfrak{K}_{2}$ & 2 \\
\hline 29 & 126 & $2 \times 3^{2} \times 7$ & 2 & $2^{4} 3^{4} 7^{4}$ & 4 & 0 & 0 & 1 & 6 & $(\times,-,-,--)$ & $\gamma$ & & 6 \\
\hline 30 & 131 & & $1 b$ & $5^{2} 131^{4}$ & 1 & 2 & 2 & 4 & 1 & $(-,-, \otimes,-)$ & $\alpha_{2}$ & $\mathcal{L}, \mathfrak{K}_{2}$ & 6 \\
\hline 31 & 132 & $2^{2} \times 3 \times 11$ & 2 & $2^{4} 3^{4} 11^{4}$ & 3 & 1 & 1 & 3 & 4 & $(-,-,(\times),-)$ & $\beta_{2}$ & $11, \mathcal{L}$ & 5 \\
\hline 32 & 133 & $7 \times 19$ & $1 \mathrm{~b}$ & $5^{2} 7^{4} 19^{4}$ & 4 & 1 & 1 & 3 & 4 & $(-, \otimes,-,-)$ & $\beta_{2}$ & $7, \mathcal{L}$ & 7 \\
\hline 33 & 139 & & $1 \mathrm{~b}$ & $5^{2} 139^{4}$ & 1 & 1 & 1 & 3 & 4 & $(-, \otimes,-,-)$ & $\beta_{2}$ & $5, \mathcal{L}$ & 4 \\
\hline 34 & 140 & $2^{2} \times 5 \times 7$ & 1a & $5^{6} 2^{4} 7^{4}$ & 16 & 1 & 2 & 4 & 5 & $(\times,-,-,-)$ & $\varepsilon$ & 7 & 5 \\
\hline 35 & 141 & $3 \times 47$ & $1 b$ & $5^{2} 3^{4} 47^{4}$ & 3 & 1 & 2 & 4 & 5 & $(\times,-,-,-)$ & $\varepsilon$ & $47 \times 5$ & 19 \\
\hline 36 & 149 & & 2 & $149^{4}$ & 1 & 1 & 1 & 2 & 3 & $(-, \otimes,-, \times)$ & $\delta_{2}$ & $\mathcal{L}$ & 6 \\
\hline
\end{tabular}




\section{Continued}

\begin{tabular}{cccccccccccccc}
\hline 37 & 151 & & 2 & $151^{4}$ & 1 & 1 & 1 & 2 & 3 & $(-,-, \otimes, \times)$ & $\alpha_{2}$ & $\mathcal{L}, \mathfrak{K}_{1}$ & 3 \\
38 & 154 & $2 \times 7 \times 11$ & $1 \mathrm{~b}$ & $5^{2} 2^{4} 7^{4} 11^{4}$ & 12 & 2 & 3 & 7 & 4 & $(-,-,(\times),-)$ & $\beta_{2}$ & $2 \times 11^{2}, \mathcal{K}$ & 3 \\
39 & 155 & $5 \times 31$ & $1 \mathrm{a}$ & $5^{6} 31^{4}$ & 4 & 2 & 3 & 5 & 2 & $(-,-, \otimes,-)$ & $\alpha_{1}$ & $\mathfrak{K}_{1}, \mathfrak{K}_{2}$ & 2 \\
40 & 171 & $3^{2} \times 19$ & $1 \mathrm{~b}$ & $5^{2} 3^{4} 19^{4}$ & 3 & 1 & 2 & 4 & 5 & $(-, \times,-,-)$ & $\varepsilon$ & $19 \times 5^{2}$ & 6 \\
41 & 174 & $2 \times 3 \times 29$ & 2 & $2^{4} 3^{4} 29^{4}$ & 3 & 1 & 1 & 3 & 4 & $(-, \otimes,-,-)$ & $\beta_{2}$ & $3^{2} \times 29, \mathcal{K}$ & 3 \\
42 & 180 & $2^{2} \times 3^{2} \times 5$ & $1 \mathrm{a}$ & $5^{6} 2^{4} 3^{4}$ & 16 & 1 & 2 & 4 & 5 & $(\times,-,-,-)$ & $\varepsilon$ & 3 & 5 \\
43 & 182 & $2 \times 7 \times 13$ & 2 & $2^{4} 7^{4} 13^{4}$ & 4 & 1 & 2 & 4 & 5 & $(\times,-,-,-)$ & $\varepsilon$ & 7 & 1 \\
44 & 186 & $2 \times 3 \times 31$ & $1 \mathrm{~b}$ & $5^{2} 2^{4} 3^{4} 31^{4}$ & 13 & 2 & 3 & 6 & 3 & $(-,-, \otimes,-)$ & $\beta_{1}$ & $5, \mathfrak{K}_{2}$ & 7 \\
45 & 190 & $2 \times 5 \times 19$ & $1 \mathrm{a}$ & $5^{6} 2^{4} 19^{4}$ & 16 & 1 & 1 & 3 & 4 & $(-, \otimes,-,-)$ & $\beta_{2}$ & $5, \mathcal{K}$ & 9 \\
46 & 191 & & $1 \mathrm{~b}$ & $5^{2} 191^{4}$ & 1 & 1 & 2 & 4 & 5 & $(-,-, \otimes,-)$ & $\beta_{1}$ & $5, \mathfrak{K}_{1}$ & 3 \\
\hline
\end{tabular}

Table 28. 44 prototypes $200<\mathbf{M}<510$ of pure metacyclic fields.

\begin{tabular}{|c|c|c|c|c|c|c|c|c|c|c|c|c|c|}
\hline No. & $\mathbf{M}$ & Factors & S & $f^{4}$ & $m$ & $V_{L}$ & $V_{M}$ & $V_{N}$ & $E$ & $(1,2,4,5)$ & $\mathrm{T}$ & $\mathrm{P}$ & $|\mathbf{M}|$ \\
\hline 47 & 202 & $2 \times 101$ & $1 \mathrm{~b}$ & $5^{2} 2^{4} 101^{4}$ & 4 & 1 & 1 & 3 & 4 & $(-, \otimes,-,-)$ & $\beta_{2}$ & $2 \times 5, \mathcal{K}$ & 6 \\
\hline 48 & 203 & $7 \times 29$ & $1 \mathrm{~b}$ & $5^{2} 7^{4} 29^{4}$ & 4 & 1 & 2 & 4 & 5 & $(-, \times,-,-)$ & $\varepsilon$ & $29 \times 5$ & 2 \\
\hline 49 & 209 & $11 \times 19$ & $1 \mathrm{~b}$ & $5^{2} 11^{4} 19^{4}$ & 3 & 2 & 3 & 7 & 4 & $(-, \otimes, \times,-)$ & $\beta_{2}$ & $11 \times 5^{2}, \mathcal{K}_{(19)}$ & 2 \\
\hline 50 & 210 & $2 \times 3 \times 5 \times 7$ & $1 \mathrm{a}$ & $5^{6} 2^{4} 3^{4} 7^{4}$ & 64 & 1 & 2 & 5 & 6 & $(\times,-,-,-)$ & $\gamma$ & $7,3^{2} \times 5$ & 5 \\
\hline 51 & 211 & & $1 \mathrm{~b}$ & $5^{2} 211^{4}$ & 1 & 3 & 5 & 9 & 2 & $(-,-, \otimes,-)$ & $\delta_{1}$ & $\mathfrak{K}_{2}$ & 1 \\
\hline 52 & 218 & $2 \times 109$ & 2 & $2^{4} 109^{4}$ & 1 & 1 & 2 & 4 & 5 & $(-, \times,-,-)$ & $\varepsilon$ & 2 & 1 \\
\hline 53 & 231 & $3 \times 7 \times 11$ & $1 \mathrm{~b}$ & $5^{2} 3^{4} 7^{4} 11^{4}$ & 12 & 1 & 2 & 5 & 6 & $(-,-, \times,-)$ & $\gamma$ & $11,7 \times 5^{2}$ & 5 \\
\hline 54 & 247 & $13 \times 19$ & $1 \mathrm{~b}$ & $5^{2} 13^{4} 19^{4}$ & 3 & 1 & 2 & 5 & 6 & $(-, \times,-,-)$ & $\gamma$ & & 2 \\
\hline 55 & 253 & $11 \times 23$ & $1 \mathrm{~b}$ & $5^{2} 11^{4} 23^{4}$ & 3 & 1 & 2 & 4 & 5 & $(-,-, \otimes,-)$ & $\beta_{1}$ & $11, \mathfrak{K}_{1}$ & 4 \\
\hline 56 & 259 & $7 \times 37$ & $1 \mathrm{~b}$ & $5^{2} 7^{4} 37^{4}$ & 4 & 1 & 2 & $5^{\star}$ & 6 & $(\times,-,-,--)$ & $\gamma$ & & 1 \\
\hline 57 & 266 & $2 \times 7 \times 19$ & $1 \mathrm{~b}$ & $5^{2} 2^{4} 7^{4} 19^{4}$ & 12 & 1 & 2 & 5 & 6 & $(-, \times,-,-)$ & $\gamma$ & $5,2^{3} \times 7$ & 3 \\
\hline 58 & 273 & $3 \times 7 \times 13$ & $1 \mathrm{~b}$ & $5^{2} 3^{4} 7^{4} 13^{4}$ & 12 & 2 & 4 & 8 & 5 & $(\times,-,-,-)$ & $\varepsilon$ & $7 \times 13^{2} \times 5^{4}$ & 4 \\
\hline 59 & 275 & $5^{2} \times 11$ & $1 \mathrm{a}$ & $5^{6} 11^{4}$ & 4 & 2 & 2 & 4 & 1 & $(-,-, \otimes,-)$ & $\alpha_{2}$ & $\mathcal{K}, \mathfrak{K}_{2}$ & 2 \\
\hline 60 & 276 & $2^{2} \times 3 \times 23$ & 2 & $2^{4} 3^{4} 23^{4}$ & 3 & 0 & 0 & 1 & 6 & $(\times,-,-,-)$ & $\gamma$ & & 10 \\
\hline 61 & 281 & & $1 \mathrm{~b}$ & $5^{2} 281^{4}$ & 1 & 3 & $5^{*}$ & $9^{*}$ & 2 & $(-,-, \otimes,-)$ & $\alpha_{1}$ & $\mathfrak{K}_{1}, \mathfrak{K}_{2}$ & 1 \\
\hline 62 & 285 & $3 \times 5 \times 19$ & 1a & $5^{6} 3^{4} 19^{4}$ & 16 & 1 & 2 & 5 & 6 & $(-, \times,-,-)$ & $\gamma$ & & 1 \\
\hline 63 & 286 & $2 \times 11 \times 13$ & $1 \mathrm{~b}$ & $5^{2} 2^{4} 11^{4} 13^{4}$ & 13 & 2 & 3 & 7 & 4 & $(-,-,(\times),-)$ & $\beta_{2}$ & $11^{2} \times 5, \mathcal{K}$ & 3 \\
\hline 64 & 287 & $7 \times 41$ & $1 \mathrm{~b}$ & $5^{2} 7^{4} 41^{4}$ & 4 & 2 & 2 & 4 & 1 & $(-,-, \otimes,-)$ & $\alpha_{2}$ & $\mathcal{K}, \mathfrak{K}_{1}$ & 1 \\
\hline 65 & 290 & $2 \times 5 \times 29$ & $1 \mathrm{a}$ & $5^{6} 2^{4} 29^{4}$ & 16 & 1 & 2 & 4 & 5 & $(-, \times,-,-)$ & $\varepsilon$ & 29 & 2 \\
\hline 66 & 298 & $2 \times 149$ & $1 \mathrm{~b}$ & $5^{2} 2^{4} 149^{4}$ & 4 & 1 & 2 & 4 & 5 & $(-, \times,-,-)$ & $\varepsilon$ & 5 & 2 \\
\hline 67 & 301 & $7 \times 43$ & 2 & $7^{4} 43^{4}$ & 4 & 0 & 0 & 1 & 6 & $(-,-,-, \otimes)$ & $\eta$ & & 1 \\
\hline 68 & 302 & $2 \times 151$ & $1 \mathrm{~b}$ & $5^{2} 2^{4} 151^{4}$ & 4 & 1 & 2 & 4 & 5 & $(-,-, \otimes,-)$ & $\beta_{1}$ & $2, \mathfrak{K}_{1}$ & 2 \\
\hline 69 & 319 & $11 \times 29$ & $1 \mathrm{~b}$ & $5^{2} 11^{4} 29^{4}$ & 3 & 2 & 2 & 5 & 2 & $(-, \otimes,(\times),-)$ & $\alpha_{3}$ & $\mathcal{K}_{(11)}, \mathcal{K}_{(29)}$ & 3 \\
\hline 70 & 329 & $7 \times 47$ & $1 \mathrm{~b}$ & $5^{2} 7^{4} 47^{4}$ & 4 & 1 & 2 & 4 & 5 & $(\times,-,-,-)$ & $\varepsilon$ & 47 & 7 \\
\hline
\end{tabular}




\section{Continued}

\begin{tabular}{|c|c|c|c|c|c|c|c|c|c|c|c|c|c|}
\hline 71 & 330 & $2 \times 3 \times 5 \times 11$ & 1a & $5^{6} 2^{4} 3^{4} 11^{4}$ & 64 & 2 & 4 & 9 & 6 & $(-,-, \times,-)$ & $\gamma$ & $3 \times 11,5 \times 11^{3}$ & 2 \\
\hline 72 & 341 & $11 \times 31$ & $1 b$ & $5^{2} 11^{4} 31^{4}$ & 3 & 3 & 5 & 9 & 2 & $(-,-, \otimes,-)$ & $\alpha_{1}$ & $\begin{array}{l}\mathfrak{K}_{(11), 1} \mathfrak{K}_{(31), 2}^{3}, \\
\mathfrak{K}_{(11), 2} \mathfrak{K}_{(31), 1}\end{array}$ & 2 \\
\hline 73 & 348 & $2^{2} \times 3 \times 29$ & $1 b$ & $5^{2} 2^{4} 3^{4} 29^{4}$ & 13 & 2 & 4 & 8 & 5 & $(-, \times,-,-)$ & $\varepsilon$ & $2 \times 3 \times 5$ & 2 \\
\hline 74 & 377 & $13 \times 29$ & $1 b$ & $5^{2} 13^{4} 29^{4}$ & 3 & 2 & 3 & 6 & 3 & $(-, \otimes,-,-)$ & $\delta_{2}$ & $\mathcal{K}$ & 1 \\
\hline 75 & 379 & & $1 b$ & $5^{2} 379^{4}$ & 1 & 1 & 2 & 4 & 5 & $(-, \times,-,-)$ & $\varepsilon$ & 5 & 1 \\
\hline 76 & 385 & $5 \times 7 \times 11$ & 1a & $5^{6} 7^{4} 11^{4}$ & 16 & 1 & 1 & 3 & 4 & $(-,-,(\times),-)$ & $\beta_{2}$ & $7 \times 11^{2}, \mathcal{K}$ & 1 \\
\hline 77 & 390 & $2 \times 3 \times 5 \times 13$ & $1 \mathrm{a}$ & $5^{6} 2^{4} 3^{4} 13^{4}$ & 64 & 1 & 2 & 5 & 6 & $(\times,-,-,-)$ & $\gamma$ & 2,5 & 4 \\
\hline 78 & 398 & $2 \times 199$ & $1 \mathrm{~b}$ & $5^{2} 2^{4} 199^{4}$ & 4 & 1 & 1 & 3 & 4 & $(-, \otimes,-,-)$ & $\beta_{2}$ & $5, \mathcal{K}$ & 7 \\
\hline 79 & 399 & $3 \times 7 \times 19$ & 2 & $3^{4} 7^{4} 19^{4}$ & 4 & 1 & 1 & 3 & 4 & $(-, \otimes,-,-)$ & $\beta_{2}$ & $3 \times 19^{2}, \mathcal{K}$ & 2 \\
\hline 80 & 401 & & 2 & $401^{4}$ & 1 & 2 & 3 & 5 & 2 & $(-,-, \otimes, \times)$ & $\alpha_{1}$ & $\mathfrak{K}_{1}, \mathfrak{K}_{2}$ & 2 \\
\hline 81 & 418 & $2 \times 11 \times 19$ & 2 & $2^{4} 11^{4} 19^{4}$ & 3 & 2 & 3 & 6 & 3 & $(-, \otimes, \otimes,-)$ & $\alpha_{2}$ & $\mathcal{K}_{(19)}, \mathfrak{K}_{(11), 1}$ & 1 \\
\hline 82 & 421 & & $1 \mathrm{~b}$ & $5^{2} 421^{4}$ & 1 & 1 & 2 & 3 & 4 & $(-,-, \otimes,-)$ & $\delta_{1}$ & $\mathfrak{K}_{2}$ & 5 \\
\hline 83 & 422 & $2 \times 211$ & $1 b$ & $5^{2} 2^{4} 211^{4}$ & 3 & 1 & 2 & 4 & 5 & $(-,-, \times,-)$ & $\varepsilon$ & $2 \times 5^{2}$ & 6 \\
\hline 84 & 451 & $11 \times 41$ & 2 & $11^{4} 41^{4}$ & 1 & 2 & 3 & 6 & 3 & $(-,-, \otimes,-)$ & $\alpha_{2}$ & $\begin{array}{l}\mathcal{K}_{(11)} \mathcal{K}_{(41)}^{4}, \\
\mathfrak{K}_{(11), 1} \mathfrak{K}_{(41), 2}^{3}\end{array}$ & 1 \\
\hline 85 & 462 & $2 \times 3 \times 7 \times 11$ & $1 b$ & $5^{2} 2^{4} 3^{4} 7^{4} 11^{4}$ & 52 & 2 & 4 & 9 & 6 & $(-,-, \times,-)$ & $\gamma$ & $\begin{array}{l}5^{2} \times 7 \\
3 \times 5 \times 11^{2}\end{array}$ & 1 \\
\hline 86 & 465 & $3 \times 5 \times 31$ & $1 \mathrm{a}$ & $5^{6} 3^{4} 31^{4}$ & 16 & 2 & 3 & $7^{\star}$ & 4 & $(-,-,(\times),-)$ & $\beta_{2}$ & $5, \mathcal{K}$ & 1 \\
\hline 87 & 473 & $11 \times 43$ & $1 \mathrm{~b}$ & $5^{2} 11^{4} 43^{4}$ & 4 & 2 & 3 & $7^{*}$ & 4 & $(-,-,(\times),-)$ & $\beta_{2}$ & $11, \mathcal{L}$ & 1 \\
\hline 88 & 482 & $2 \times 241$ & 2 & $2^{4} 241^{4}$ & 1 & 1 & 2 & 4 & 5 & $(-,-, \otimes,-)$ & $\beta_{1}$ & $241, \mathfrak{K}_{2}$ & 2 \\
\hline 89 & 502 & $2 \times 251$ & $1 b$ & $5^{2} 2^{4} 251^{4}$ & 4 & $\star^{*}$ & ${ }^{*} 4$ & $\star 8$ & 5 & $(-,-, \otimes,-)$ & $\beta_{1}$ & $251 \times 5, \mathfrak{K}_{2}$ & 1 \\
\hline 90 & 505 & $5 \times 101$ & 1a & $5^{6} 101^{4}$ & 4 & 1 & 1 & 3 & 4 & $(-,-,(\times), \otimes)$ & $\zeta_{2}$ & $\mathcal{K}$ & 2 \\
\hline
\end{tabular}

Table 29. 39 prototypes $510<\mathbf{M}<900$ of pure metacyclic fields.

\begin{tabular}{cccccccccccccc}
\hline No. & M & Factors & $\mathrm{S}$ & $f^{4}$ & $m$ & $V_{L}$ & $V_{M}$ & $V_{N} E$ & $(1,2,4,5)$ & $\mathrm{T}$ & $\mathrm{P}$ & $|\mathbf{M}|$ \\
\hline 91 & 517 & $11 \times 47$ & $1 \mathrm{~b}$ & $5^{2} 11^{4} 47^{4}$ & 3 & 2 & 3 & 6 & 3 & $(-,-, \otimes,-)$ & $\beta_{1}$ & $11 \times 5^{2}, \mathfrak{K}_{2}$ & 1 \\
92 & 532 & $2^{2} \times 7 \times 19$ & 2 & $2^{4} 7^{4} 19^{4}$ & 4 & 1 & 2 & 4 & 5 & $(-, \times,-,-)$ & $\varepsilon$ & $2 \times 7$ & 1 \\
93 & 546 & $2 \times 3 \times 7 \times 13$ & $1 \mathrm{~b}$ & $5^{2} 2^{4} 3^{4} 7^{4} 13^{4}$ & 52 & 2 & 4 & 9 & 6 & $(\times,-,-,-)$ & $\gamma$ & $3 \times 5 \times 13$, & 3 \\
94 & 550 & $2 \times 5^{2} \times 11$ & $1 \mathrm{a}$ & $5^{6} 2^{4} 11^{4}$ & 16 & 2 & 3 & 6 & 3 & $(-,-, \otimes,-)$ & $\alpha_{2}$ & $\mathcal{K}_{,}, \mathfrak{K}_{1}$ & 1 \\
95 & 551 & $19 \times 29$ & 2 & $19^{4} 29^{4}$ & 1 & 2 & 2 & 5 & 2 & $(-, \otimes,-,-)$ & $\alpha_{3}$ & $\mathcal{K}_{(19)}, \mathcal{K}_{(29)}$ & 1 \\
96 & 570 & $2 \times 3 \times 5 \times 19$ & $1 \mathrm{a}$ & $5^{6} 2^{4} 3^{4} 19^{4}$ & 64 & 1 & 2 & 5 & 6 & $(-, \times,-,-)$ & $\gamma$ & $2^{4} \times 3,2^{4} \times 5$ & 2 \\
97 & 574 & $2 \times 7 \times 41$ & 2 & $2^{4} 7^{4} 41^{4}$ & 4 & 1 & 1 & 3 & 4 & $(-,-,(\times),-)$ & $\beta_{2}$ & $41, \mathcal{L}$ & 3 \\
98 & 590 & $2 \times 5 \times 59$ & $1 \mathrm{a}$ & $5^{6} 2^{4} 59^{4}$ & 16 & 2 & 3 & $* 7$ & 4 & $(-, \otimes,-,-)$ & $\beta_{2}$ & $5, \mathcal{K}$ & 1 \\
99 & 602 & $2 \times 7 \times 43$ & $1 \mathrm{~b}$ & $5^{2} 2^{4} 7^{4} 43^{4}$ & 16 & 1 & 2 & 5 & 6 & $(\times,-,-,-)$ & $\gamma$ & 2,7 & 2 \\
100 & 606 & $2 \times 3 \times 101$ & $1 \mathrm{~b}$ & $5^{2} 2^{4} 3^{4} 101^{4}$ & 12 & 1 & 2 & 5 & 6 & $(-,-, \times,-)$ & $\gamma$ & $2 \times 5,101$ & 2 \\
101 & 609 & $3 \times 7 \times 29$ & $1 \mathrm{~b}$ & $5^{2} 3^{4} 7^{4} 29^{4}$ & 12 & 2 & 3 & 7 & 4 & $(-, \otimes,-,-)$ & $\beta_{2}$ & $7^{2} \times 5, \mathcal{K}$ & 1 \\
102 & 620 & $2 \times 5 \times 31$ & $1 \mathrm{a}$ & $5^{6} 2^{4} 31^{4}$ & 16 & 2 & $4^{*}$ & $8^{*}$ & 5 & $(-,-, \otimes,-)$ & $\beta_{1}$ & $5, \mathfrak{K}_{1}$ & 1 \\
\hline
\end{tabular}




\section{Continued}

\begin{tabular}{|c|c|c|c|c|c|c|c|c|c|c|c|c|}
\hline 103 & 627 & $3 \times 11 \times 19$ & $1 b$ & $5^{2} 3^{4} 11^{4} 19^{4}$ & 13 & 3 & 4 & 9 & $2(-, \otimes,(\times),-)$ & $\alpha_{3}$ & $\mathcal{K}_{(11)}, \mathcal{K}_{(19)}$ & 1 \\
\hline 104 & 638 & $2 \times 11 \times 29$ & $1 b$ & $5^{2} 2^{4} 11^{4} 29^{4}$ & 13 & 2 & 3 & 7 & $4(-, \otimes,(\times),-)$ & $\beta_{2}$ & $\begin{array}{l}2^{4} \times 11 \times 5 \\
\mathcal{K}_{(11)} \times \mathcal{K}_{(29)}^{4}\end{array}$ & 2 \\
\hline 105 & 649 & $11 \times 59$ & 2 & $11^{4} 59^{4}$ & 1 & 2 & 2 & 5 & $2(-, \otimes,(\times),-)$ & $\alpha_{3}$ & $\mathcal{K}_{(11)}, \mathcal{K}_{(59)}$ & 2 \\
\hline 106 & 660 & $2^{2} \times 3 \times 5 \times 11$ & $1 \mathrm{a}$ & $5^{6} 2^{4} 3^{4} 11^{4}$ & 64 & 1 & 2 & 5 & $6 \quad(-,-, \times,-)$ & $\gamma$ & $2 \times 5,5 \times 11$ & 2 \\
\hline 107 & 665 & $5 \times 7 \times 19$ & 1a & $5^{6} 7^{4} 19^{4}$ & 16 & 1 & 1 & 3 & $4 \quad(-, \otimes,-,-)$ & $\beta_{2}$ & $7, \mathcal{K}$ & 1 \\
\hline 108 & 671 & $11 \times 61$ & $1 b$ & $5^{2} 11^{4} 61^{4}$ & 3 & 3 & 4 & 8 & $1 \quad(-,-, \otimes,-)$ & $\alpha_{2}$ & $\begin{array}{l}\mathcal{K}_{(11)} \mathcal{K}_{(61)}, \\
\mathfrak{K}_{(11), 2} \mathfrak{K}_{(61), 1}^{2}\end{array}$ & 1 \\
\hline 109 & 682 & $2 \times 11 \times 31$ & 2 & $2^{4} 11^{4} 31^{4}$ & 3 & 2 & 3 & 6 & $3 \quad(-,-, \otimes,-)$ & $\alpha_{2}$ & $\begin{array}{l}\mathcal{K}_{(11)} \mathcal{K}_{(31)}^{3}, \\
\mathfrak{K}_{(11), !} \mathfrak{K}_{(31), 2}^{3}\end{array}$ & 1 \\
\hline 110 & 691 & & $1 b$ & $5^{2} 691^{4}$ & 1 & 3 & 4 & 8 & $1 \quad(-,-, \otimes,-)$ & $\alpha_{2}$ & $\mathcal{L}, \mathfrak{K}_{2}$ & 1 \\
\hline 111 & 693 & $3^{2} \times 7 \times 11$ & 2 & $3^{4} 7^{4} 11^{4}$ & 4 & 2 & 3 & 6 & $3 \quad(-,-, \otimes,-)$ & $\beta_{1}$ & $3 \times 7, \mathfrak{K}_{2}$ & 1 \\
\hline 112 & 695 & $5 \times 139$ & $1 \mathrm{a}$ & $5^{6} 139^{4}$ & 4 & 1 & 2 & 4 & $5 \quad(-, \times,-,-)$ & $\varepsilon$ & 139 & 1 \\
\hline 113 & 702 & $2 \times 3^{3} \times 13$ & $1 b$ & $5^{2} 2^{4} 3^{4} 13^{4}$ & 13 & 2 & 4 & 8 & $5 \quad(\times,-,-,-)$ & $\varepsilon$ & $13 \times 5^{4}$ & 2 \\
\hline 114 & 707 & $7 \times 101$ & 2 & $7^{4} 101^{4}$ & 4 & 1 & 1 & 3 & $4(-,-,(\times), \otimes)$ & $\zeta_{2}$ & $\mathcal{K}$ & 1 \\
\hline 115 & 710 & $2 \times 5 \times 71$ & 1a & $5^{6} 2^{4} 71^{4}$ & 16 & 2 & 2 & 4 & $1 \quad(-,-, \otimes,-)$ & $\alpha_{2}$ & $\mathcal{K}, \mathfrak{K}_{2}$ & 4 \\
\hline 116 & 745 & $5 \times 149$ & $1 \mathrm{a}$ & $5^{6} 149^{4}$ & 4 & 1 & 1 & 3 & $4 \quad(-, \otimes,-, \otimes)$ & $\zeta_{2}$ & $\mathcal{K}$ & 2 \\
\hline 117 & 749 & $7 \times 107$ & 2 & $7^{4} 107^{4}$ & 4 & 1 & 2 & 4 & $5 \quad(-,-,-, \times)$ & $\varepsilon$ & & 1 \\
\hline 118 & 751 & & 2 & $751^{4}$ & 1 & 2 & 2 & 4 & $1 \quad(-,-, \otimes, \times)$ & $\alpha_{2}$ & $\mathcal{L}, \mathfrak{K}_{1}$ & 1 \\
\hline 119 & 770 & $2 \times 5 \times 7 \times 11$ & 1a & $5^{6} 2^{4} 7^{4} 11^{4}$ & 64 & 1 & 2 & 5 & $6 \quad(-,-, \times,-)$ & $\gamma$ & $2 \times 5,2^{2} \times 11$ & 1 \\
\hline 120 & 779 & $19 \times 41$ & $1 b$ & $5^{2} 19^{4} 41^{4}$ & 3 & 3 & 4 & 8 & $1 \quad(-, \otimes, \otimes,-)$ & $\alpha_{2}$ & $\begin{array}{l}\mathcal{K}_{(19)} \mathcal{K}_{(41)}, \\
\mathfrak{K}_{(41), 1}\end{array}$ & 1 \\
\hline 121 & 785 & $5 \times 157$ & $1 \mathrm{a}$ & $5^{6} 157^{4}$ & 4 & 1 & 2 & 4 & $5 \quad(-,-,-, \times)$ & $\varepsilon$ & & 1 \\
\hline 122 & 798 & $2 \times 3 \times 7 \cdot 19$ & $1 \mathrm{~b}$ & $5^{2} 2^{4} 3^{4} 7^{4} 19^{4}$ & 52 & 2 & 4 & 9 & $6 \quad(-, \times,-,-)$ & $\gamma$ & $3 \times 19,7 \times 5^{4}$ & 1 \\
\hline 123 & 808 & $2^{3} \times 101$ & $1 b$ & $5^{2} 2^{4} 101^{4}$ & 4 & 2 & 2 & 4 & $1 \quad(-,-, \otimes,-)$ & $\alpha_{2}$ & $\mathcal{K}, \mathfrak{K}_{1}$ & 2 \\
\hline 124 & 825 & $3 \times 5^{2} \times 11$ & 1a & $5^{6} 3^{4} 11^{4}$ & 16 & 1 & 2 & 4 & $5 \quad(-,-, \otimes,-)$ & $\beta_{1}$ & $3^{2} \times 11, \mathfrak{K}_{1}$ & 1 \\
\hline 125 & 843 & $3 \times 281$ & 2 & $3^{4} 281^{4}$ & 1 & 1 & 2 & 3 & $4 \quad(-,-, \otimes,-)$ & $\delta_{1}$ & $\mathfrak{K}_{1}$ & 1 \\
\hline 126 & 858 & $2 \times 3 \times 11 \times 13$ & $1 b$ & $5^{2} 2^{4} 3^{4} 11^{4} 13^{4}$ & 51 & 2 & 4 & 9 & $6 \quad(-,-, \times,-)$ & $\gamma$ & $2 \times 5,13 \times 5$ & 1 \\
\hline 127 & 861 & $3 \times 7 \times 41$ & $1 b$ & $5^{2} 3^{4} 7^{4} 41^{4}$ & 13 & 3 & 4 & 8 & $1 \quad(-,-, \otimes,-)$ & $\alpha_{2}$ & $\mathcal{K}, \mathfrak{K}_{1}$ & 1 \\
\hline 128 & 893 & $19 \times 47$ & 2 & $19^{4} 47^{4}$ & 1 & 1 & 1 & 3 & $4 \quad(-, \otimes,-,-)$ & $\beta_{2}$ & $19, \mathfrak{L}$ & 1 \\
\hline 129 & 894 & $2 \times 3 \times 149$ & $1 b$ & $5^{2} 2^{4} 3^{4} 149^{4}$ & 12 & 1 & 2 & 5 & $6 \quad(-, \times,-,-)$ & $\gamma$ & $5,2 \times 3^{2}$ & 1 \\
\hline
\end{tabular}

exceptional number $t=0$ of primes $q \neq 5$ dividing the conductor $f$ (here $\left.f^{4}=5^{6}\right)$. The invariants of this unique metacyclic Polya field $N$ are given by

[5], species 1a, $\left(e_{0} ; t, u, v, m ; n, s_{2}, s_{4}\right)=(6 ; 0,0,0,1 ; 0,0,0)$,

type $\vartheta,(U, \eta, \zeta ; A, I, R)=(0, \times, \times ; 1,0,0)$, and $\left(V_{L}, V_{M}, V_{N} ; E\right)=(0,0,0 ; 5) .(4.4)$

We conjecture that all the other similarity classes are infinite. Precisely four of them can actually be given by parametrized infinite sequences in a deterministic way aside from the intrinsic probabilistic nature of the occurrence of primes in 
Table 30. 5 prototypes $900<\mathbf{M}<1000$ of pure metacyclic fields.

\begin{tabular}{cccccccccccccc}
\hline No. & $\mathbf{M}$ & Factors & $\mathrm{S}$ & $f^{4}$ & $m$ & $V_{L}$ & $V_{M}$ & $V_{N}$ & $E$ & $(1,2,4,5)$ & $\mathrm{T}$ & $\mathrm{P}$ & $|\mathbf{M}|$ \\
\hline 130 & 902 & $2 \times 11 \times 41$ & $1 \mathrm{~b}$ & $5^{2} 2^{4} 11^{4} 41^{4}$ & 13 & 2 & 3 & 7 & 4 & $(-,-,(\times),-)$ & $\beta_{2}$ & $11 \times 5, \mathcal{K}_{(11)} \times \mathcal{K}_{(41)}$ & 1 \\
131 & 924 & $2^{2} \times 3 \times 7 \times 11$ & 2 & $2^{4} 3^{4} 7^{4} 11^{4}$ & 12 & 1 & 2 & 5 & 6 & $(-,-, \times,-)$ & $\gamma$ & $3 \times 7,7 \times 11$ & 1 \\
132 & 955 & $5 \times 191$ & $1 \mathrm{a}$ & $5^{6} 191^{4}$ & 4 & $2^{*}$ & $3^{*}$ & $6^{*}$ & 3 & $(-,-, \otimes,-)$ & $\alpha_{2}$ & $\mathcal{K}_{,}, \mathfrak{K}_{1}$ & 1 \\
133 & 957 & $3 \times 11 \times 29$ & 2 & $3^{4} 11^{4} 29^{4}$ & 3 & 2 & 2 & 5 & 2 & $(-, \otimes,(\times),-)$ & $\alpha_{3}$ & $\mathcal{K}_{(11)}, \mathcal{K}_{(29)}$ & 1 \\
134 & 982 & $2 \times 491$ & 2 & $2^{4} 491^{4}$ & 1 & 1 & 1 & 2 & 3 & $(-,-, \otimes,-)$ & $\alpha_{2}$ & $\mathcal{K}_{,}, \mathfrak{K}_{1}$ & 2 \\
\hline
\end{tabular}

residue classes and of composite integers with assigned shape of prime decomposition. This was proved in ([6] Thm. 10.1)and ([7] Thm. 2.1).

Theorem 4.1 Each of the following infinite sequences of conductors $f=f_{N / K}$ unambiguously determines the DPF type of the pure metacyclic fields $N$ in the associated multiplet with $m=m(f)$ members.

1) $f=q$ with $q \in \mathbb{P}, q \equiv \pm 7 \bmod (25)$ gives rise to a singulet, $m=1$, with DPF type $\vartheta$,

2) $f^{4}=5^{2} \cdot q^{4}$ with $q \in \mathbb{P}, q \equiv \pm 2 \bmod (5), q \equiv \pm 7 \bmod (25)$ gives rise to a singulet, $m=1$, with DPF type $\varepsilon$,

3) $f^{4}=5^{6} \cdot q^{4}$ with $q \in \mathbb{P}, q \equiv \pm 2 \bmod (5), q \neq \equiv \pm 7 \bmod (25)$ gives rise to a quartet, $m=4$, with homogeneous DPF type $(\varepsilon, \varepsilon, \varepsilon, \varepsilon)$,

4) $f=q_{1} \cdot q_{2}$ with $q_{i} \in \mathbb{P}, q_{i} \equiv \pm 2 \bmod (5), q_{i} \equiv \pm \pm \bmod (25)$ gives rise to a singulet, $m=1$, with DPF type $\varepsilon$.

In fact, the shape of the conductors in Theorem 4.1 does not only determine the refined Dedekind species and the DPF type, but also the structure of the 5-class groups of the fields $L, M$ and $N$.

Corollary 4.1 The invariants of the similarity classes defined by the four infinite sequences of conductors in Theorem 4.1 are given as follows, in the same order:

[7], species $2, \quad\left(e_{0} ; t, u, v, m ; n, s_{2}, s_{4}\right)=(0 ; 1,1,0,1 ; 1,0,0)$,

type $\vartheta,(U, \eta, \zeta ; A, I, R)=(0, \times, \times ; 1,0,0)$, and $\left(V_{L}, V_{M}, V_{N} ; E\right)=(0,0,0 ; 5) ;($

[2], species $1 b,\left(e_{0} ; t, u, v, m ; n, s_{2}, s_{4}\right)=(2 ; 1,0,1,1 ; 1,0,0)$,

type $\varepsilon,(U, \eta, \zeta ; A, I, R)=(1, \times,-; 2,0,0)$, and $\left(V_{L}, V_{M}, V_{N} ; E\right)=(0,0,0 ; 5) ;$

[10], species 1a, $\left(e_{0} ; t, u, v, m ; n, s_{2}, s_{4}\right)=(6 ; 1,0,1,4 ; 1,0,0)$,

type $\varepsilon,(U, \eta, \zeta ; A, I, R)=(1, \times,-; 2,0,0)$, and $\left(V_{L}, V_{M}, V_{N} ; E\right)=(0,0,0 ; 5) ;(4.7)$

[18], species $2, \quad\left(e_{0} ; t, u, v, m ; n, s_{2}, s_{4}\right)=(0 ; 2,0,2,1 ; 2,0,0)$,

type $\varepsilon,(U, \eta, \zeta ; A, I, R)=(1, \times,-; 2,0,0)$, and $\left(V_{L}, V_{M}, V_{N} ; E\right)=(0,0,0 ; 5)$.

The pure metacyclic fields $N$ associated with these four similarity classes are Polya fields.

Remark 4.1 The statements concerning 5-class groups in Corollary 4.1 were proved by Parry in ([1] Thm. IV, p. 481), where Formula (10) gives the shape of radicands associated with the conductors in our Theorem 4.1. 
Proof. (of Theorem 4.1 and Corollary 4.1) It only remains to show the claims for the composite radicands associated with conductors $f^{4}=5^{6} \cdot q^{4}$ and $f=q_{2} \cdot q_{2}$. See ([6] Thm. 10.6).

For similarity classes distinct from the four infinite classes in Theorem 4.1 we cannot provide deterministic criteria for the DPF type and for the homogeneity of multiplets with $m>1$. In general, the members of a multiplet belong to distinct similarity classes, thus giving rise to heterogeneous DPF types. We explain these phenomena with the simplest cases where only two or three DPF types are involved (type splitting).

Theorem 4.2 Each of the following infinite sequences of conductors $f=f_{N / K}$ admits precisely three DPF types of the pure metacyclic fields $N$ in the associated quartet with $m=4$ members.

1) $f^{4}=5^{6} \cdot q^{4}$ with $q \in \mathbb{P}, q \equiv \pm 7 \bmod (25)$ gives rise to a quartet with possibly heterogeneous DPF type $\left(\varepsilon^{x}, \eta^{y}, \vartheta^{z}\right), x+y+z=4$, conjecturally always $z=0$,

2) $f=q_{2} \cdot q_{2}$ with $q_{i} \in \mathbb{P}, q_{i} \equiv \pm 7 \bmod (25)$ gives rise to a quartet with possibly heterogeneous DPF type $\left(\varepsilon^{x}, \eta^{y}, \vartheta^{z}\right), x+y+z=4$, conjecturally always $z=0$.

Example 4.1 It is quite easy to find complete quartets, whose members are spread rather widely. The smallest quartet

$(35,175,245,4375)=\left(5 \times 7,5^{2} \times 7,5 \times 7^{2}, 5^{4} \times 7\right)$ belonging to the first infinite sequence contains the member $D=4375$ outside of the range of our systematic computations. We have determined its DPF type separately and thus discovered a homogeneous quartet of type $(\eta, \eta, \eta, \eta)$. However, we cannot generally exclude the occurrence of heterogeneous quartets.

Corollary 4.2 The invariants of the similarity classes defined by the two infinite sequences of conductors in Theorem 4.2 are given as follows, in the same order. The statements concerning 5-class groups are only conjectural. Each sequence splits in two similarity classes.

The classes for $f^{4}=5^{6} \cdot q^{4}$ are:

[35], species $1 a,\left(e_{0} ; t, u, v, m ; n, s_{2}, s_{4}\right)=(6 ; 1,1,0,4 ; 1,0,0)$,

type $\eta,(U, \eta, \zeta ; A, I, R)=(1,-, \times ; 2,0,0)$, and $\left(V_{L}, V_{M}, V_{N} ; E\right)=(0,0,1 ; 6)$;

[785], species $1 a,\left(e_{0} ; t, u, v, m ; n, s_{2}, s_{4}\right)=(6 ; 1,1,0,4 ; 1,0,0)$,

type $\varepsilon,(U, \eta, \zeta ; A, I, R)=(1, \times,-; 2,0,0)$, and $\left(V_{L}, V_{M}, V_{N} ; E\right)=(1,2,4 ; 5)$.

The classes for $f=q_{1} \cdot q_{2}$ are:

[301], species $2, \quad\left(e_{0} ; t, u, v, m ; n, s_{2}, s_{4}\right)=(0 ; 2,2,0,4 ; 2,0,0)$,

type $\eta,(U, \eta, \zeta ; A, I, R)=(1,-, \times ; 2,0,0)$, and $\left(V_{L}, V_{M}, V_{N} ; E\right)=(0,0,1 ; 6)$;

[749], species $2, \quad\left(e_{0} ; t, u, v, m ; n, s_{2}, s_{4}\right)=(0 ; 2,2,0,4 ; 2,0,0)$, 
type $\varepsilon,(U, \eta, \zeta ; A, I, R)=(1, \times,-; 2,0,0)$, and $\left(V_{L}, V_{M}, V_{N} ; E\right)=(1,2,4 ; 5)$.

All pure metacyclic fields $N$ associated with these four similarity classes are Polya fields.

Proof. (of Theorem 4.2 and Corollary 4.2) We use $T=2, v=s_{2}=s_{4}=0$ and ([6] Thm. 6.1).

Remark 4.2. The statements on 5-class groups in Corollary 4.2 have been verified for all examples with $2 \leq D<1000$ by our computations. In particular, the occurrence of the radicands $D=749=7 \times 107$ and $D=785=5 \times 157$, both with $V_{L}=1$, proves the impossibility of the general claim $5 \nmid h(L)$ for the two situations mentioned in ([18] Lem. 3.3 (ii) and (iv), p. 204) and ([15] Thm. 5 (ii) and (iv), p. 5), partially also indicated in ([1] Thm. IV (11), p. 481).

Theorem 4.3 Each of the following infinite sequences of conductors $f=f_{N / K}$ admits precisely two DPF types of the pure metacyclic fields $\mathrm{N}$ in the associated hexadecuplet with $m=16$ members.

1) $f^{4}=5^{6} \cdot q_{1}^{4} q_{2}^{4} \quad$ with $q_{i} \in \mathbb{P}, \quad q_{i} \equiv \pm 2 \bmod (5)$, both $q_{i} \equiv \pm \pm 7 \bmod (25)$ gives rise to a hexadecuplet with possibly heterogeneous DPF type $\left(\varepsilon^{x}, \gamma^{y}\right)$, $x+y=16$,

2) $f^{4}=5^{6} \cdot q_{1}^{4} q_{2}^{4}$ with $q_{i} \in \mathbb{P}, q_{i} \equiv \pm 2 \bmod (5)$, only one $q_{i} \equiv \pm 7 \bmod (25)$ gives rise to a hexadecuplet with possibly heterogeneous DPF type $\left(\varepsilon^{x}, \gamma^{y}\right)$, $x+y=16$.

Example 4.2 It is not difficult to find complete hexadecuplets, whose members are spread rather widely. The smallest hexadecuplet

$$
\begin{aligned}
&(30,60,90,120,150,180,240,270,360,540,600,720,810,1350,1620,3750) \\
&=\left(2 \times 3 \times 5,2^{2} \times 3 \times 5,2 \times 3^{2} \times 5,2^{3} \times 3 \times 5,2 \times 3 \times 5^{2}, 2^{2} \times 3^{2} \times 5,\right. \\
& 2^{4} \times 3 \times 5,2 \times 3^{3} \times 5,2^{3} \times 3^{2} \times 5,2^{2} \times 3^{3} \times 5,2^{3} \times 3 \times 5^{2}, 2^{4} \times 3^{2} \times 5, \\
&\left.2 \times 3^{4} \times 5,2 \times 3^{3} \times 5^{2}, 2^{2} \times 3^{4} \times 5,2 \times 3 \times 5^{4}\right)
\end{aligned}
$$

belonging to the first infinite sequence contains the members $D=1350,1620,3750$ outside of the range of our systematic computations. We have determined their DPF type separately and thus discovered a heterogeneous hexadecuplet (in the same order) of type

$$
\left(\varepsilon^{3}, \gamma^{13}\right)=(\gamma, \gamma, \gamma, \gamma, \gamma, \varepsilon, \varepsilon, \gamma, \gamma, \gamma, \gamma, \gamma, \gamma, \varepsilon, \gamma, \gamma) \text {. }
$$

Corollary 4.3 The invariants of the similarity classes defined by the two infinite sequences of conductors in Theorem 4.3 are given as follows, in the same order. The statements concerning 5-class groups are only conjectural. Each sequence splits into two similarity classes.

The classes for $f^{4}=5^{6} \cdot q_{1}^{4} q_{2}^{4}$, both $q_{i} \neq \equiv \pm 7 \bmod (25)$ are:

[30], species 1 a, $\left(e_{0} ; t, u, v, m ; n, s_{2}, s_{4}\right)=(6 ; 2,0,2,16 ; 2,0,0)$,

type $\gamma,(U, \eta, \zeta ; A, I, R)=(2,-,-; 3,0,0)$, and $\left(V_{L}, V_{M}, V_{N} ; E\right)=(0,0,1 ; 6)$;

[180], species $1 a,\left(e_{0} ; t, u, v, m ; n, s_{2}, s_{4}\right)=(6 ; 2,0,2,16 ; 2,0,0)$, 
type $\varepsilon,(U, \eta, \zeta ; A, I, R)=(1, \times,-; 2,0,0)$, and $\left(V_{L}, V_{M}, V_{N} ; E\right)=(1,2,4 ; 5)$.

The classes for $f^{4}=5^{6} \cdot q_{1}^{4} q_{2}^{4}$, only one $q_{i} \equiv \pm 7 \bmod (25)$ are:

[70], species $1 a,\left(e_{0} ; t, u, v, m ; n, s_{2}, s_{4}\right)=(6 ; 2,1,1,16 ; 2,0,0)$,

type $\gamma,(U, \eta, \zeta ; A, I, R)=(2,-,-; 3,0,0)$, and $\left(V_{L}, V_{M}, V_{N} ; E\right)=(0,0,1 ; 6)$;

[140], species $1 a,\left(e_{0} ; t, u, v, m ; n, s_{2}, s_{4}\right)=(6 ; 2,1,1,16 ; 2,0,0)$,

type $\varepsilon,(U, \eta, \zeta ; A, I, R)=(1, \times,-; 2,0,0)$, and $\left(V_{L}, V_{M}, V_{N} ; E\right)=(1,2,4 ; 5)$.

Only the pure metacyclic fields $N$ of type $\gamma$ associated with (14) and (16) are Polya fields.

Proof. (of Theorem 4.3 and Corollary 4.3) See ([6] Thm. 10.7).

Theorem 4.4 A pure metacyclic field $N=\mathbb{Q}\left(\zeta_{5}, \sqrt[5]{\ell}\right)$ with prime radicand $\ell \equiv \pm 1 \bmod (25)$ has a prime conductor $f=\ell$, and possesses the Polya property, regardless of its DPF type and the complexity of its 5-class group structure.

Proof. This is an immediate consequence of ([6] Thm. 10.5 and Thm. 6.1), taking into account that we have the value $t=1$ for the number of primes dividing the conductor in the present situation, and thus the estimate in ([6] Cor. 4.1) yields $1 \leq A \leq \min (3, t)=\min (3,1)=1$. For the Polya property we must have $A=t=1$, according to ([6] Thm. 10.5), which admits the DPF types $\alpha_{1}, \alpha_{2}, \alpha_{3}, \delta_{1}, \delta_{2}, \zeta_{1}, \zeta_{2}$ or $\vartheta$ ([6] Thm. 1.3 and Tbl. 1). However, DPF type $\alpha_{3}$ is excluded by ([6] Cor. 4.2), since the requirement $s_{2}+s_{4} \geq 2$ cannot be fulfilled in our situation where either $s_{2}=0$ and $s_{4}=1$ for $\ell \equiv+1 \bmod (25)$ or $s_{2}=1$ and $s_{4}=0$ for $\ell \equiv-1 \bmod (25)$.

Theorem 4.5 A pure metacyclic field $N=\mathbb{Q}\left(\zeta_{5}, \sqrt[5]{\ell}\right)$ with prime radicand $\ell \equiv \pm 1 \bmod (5)$ but $\ell \neq \equiv \pm 1 \bmod (25)$ has a composite conductor $f^{4}=5^{2} \cdot \ell^{4}$, and the following conditions are equivalent:

1) $N$ possesses the Polya property.

2) $(\exists \alpha \in L=\mathbb{Q}(\sqrt[5]{\ell})) N_{L / \mathbb{Q}}(\alpha)=5$.

3) The prime ideal $\mathfrak{p} \in \mathbb{P}_{L}$ with $5 \mathcal{O}_{L}=\mathfrak{p}^{5}$ is principal.

4) $N$ is of DPF type either $\beta_{1}$ or $\beta_{2}$ or $\varepsilon$.

Proof. This is a consequence of ([6] Thm. 10.5 and Thm. 6.1), taking into account that the prime 5 is not included in the current definition of the counter $t$ (with value $t=1$ in the present situation), and thus the estimate in ([6] Cor. 4.1) must be replaced by $1 \leq A \leq \min (3, t+1)=\min (3,2)=2$. For the Polya property we must have $A=t+1=2$ ([6] Thm. 10.5), which determines the DPF types $\beta_{1}, \beta_{2}, \varepsilon$ or $\eta$ ([6] Thm. 1.3 and Tbl. 1). However, DPF type $\eta$ is excluded by the prime $\ell \neq \equiv \pm 1, \pm 7 \bmod (25)$ dividing the conductor (([6] Thm. 8.1)).

Inspired by the last two theorems, it is worth ones while to summarize, for each kind of prime radicands, what is known about the possibilities for 
differential principal factorizations.

Theorem 4.6 Let $N=\mathbb{Q}\left(\zeta_{5}, \sqrt[5]{D}\right)$ be a pure metacyclic field with prime radicand $D \in \mathbb{P}$.

1) If $D=q$ with $q \equiv \pm 7 \bmod (25)$ or $q=5$, then $N$ is of type $\vartheta$.

2) If $D=\ell$ with $\ell \equiv-1 \bmod (25)$, then $N$ is of one of the types $\delta_{2}, \zeta_{2}, \vartheta$.

3) If $D=\ell$ with $\ell \equiv+1 \bmod (25)$, then $N$ is of one of the types $\alpha_{1}, \alpha_{2}, \delta_{1}, \delta_{2}, \zeta_{1}, \zeta_{2}, \vartheta$.

4) If $D=q$ with $q \equiv \pm 2 \bmod (5)$ but $q \neq \equiv \pm 7 \bmod (25)$, then $N$ is of type $\varepsilon$.

5) If $D=\ell$ with $\ell \equiv-1 \bmod (5)$ but $\ell \neq \equiv-1 \bmod (25)$, then $N$ is of one of the types $\beta_{2}, \delta_{2}, \varepsilon$.

6) If $D=\ell$ with $\ell \equiv+1 \bmod (5)$ but $\ell \equiv \equiv+1 \bmod (25)$, then $N$ is of one of the types $\alpha_{1}, \alpha_{2}, \beta_{1}, \beta_{2}, \delta_{1}, \delta_{2}, \varepsilon$.

A pure metacyclic field with prime radicand can never be of any of the types $\alpha_{3}, \gamma, \eta$.

Proof. By making use of the bounds [6] $\$ 4$ for $\mathbb{F}_{5}$-dimensions of spaces of differential principal factors (DPF),

$$
\begin{aligned}
& 1 \leq A \leq \min (3, t), \\
& 0 \leq I \leq \min \left(2,2\left(s_{2}+s_{4}\right)\right), \\
& 0 \leq R \leq \min \left(2,4 s_{4}\right),
\end{aligned}
$$

we can determine the possible DPF types of pure metacyclic fields $N=\mathbb{Q}\left(\zeta_{5}, \sqrt[5]{D}\right)$ with prime radicands $D \in \mathbb{P}$. We start with a few general observations.

Firstly, if $D \equiv \pm 1, \pm 7 \bmod (25)$, resp. $D=5$, is prime, then $N$ is of Dedekind species 2 , resp. 1a, with prime power conductor $f=D$, resp. $f^{4}=5^{6}$, and $t=1$, whence $A=1$ and the types $\beta_{1}, \beta_{2}, \gamma, \varepsilon, \eta$ with $A \geq 2$ are forbidden. However, if $D \neq \pm 1, \pm 7 \bmod (25)$ and $D \neq 5$ is prime, then the congruence requirement eliminates the types $\zeta_{1}, \zeta_{2}, \eta, \vartheta$, the field $N$ is of Dedekind species $1 \mathrm{~b}$ with composite conductor $f^{4}=5^{2} \cdot D^{4}$, and $t=2$, whence $1 \leq A \leq 2$ and type $\gamma$ with $A=3$ is discouraged. So, the types $\gamma$ and $\eta$ are generally forbidden for prime radicands.

Secondly, for a prime radicand $D \equiv \pm 1 \bmod (5)$ which splits in $M$, the space of radicals $\Delta=\langle\sqrt[5]{D}\rangle$ is a 1-dimensional subspace of absolute DPF contained in the 2-dimensional space $\Delta \oplus \Delta^{\prime}$ of differential factors generated by the two prime ideals of $M$ over $D$. Consequently, in this special situation there arises an additional constraint $I \leq 1$ for the dimension of the space of intermediate DPF, which must be contained in the 1-dimensional complement $\Delta^{\prime}$. This generally excludes type $\alpha_{3}$ with $I=2$ for prime radicands.

1) If $D=q$ with $q \equiv \pm 7 \bmod (25)$, then $t=1, s_{2}=s_{4}=0$, and thus $A=1$, $I=R=0$. These conditions eliminate the types

$\alpha_{1}, \alpha_{2}, \alpha_{3}, \beta_{1}, \beta_{2}, \gamma, \delta_{1}, \delta_{2}, \varepsilon, \zeta_{1}, \zeta_{2}, \eta$ with either $A \geq 2$ or $I \geq 1$ or $R \geq 1$, and only type $\vartheta$ remains admissible.

2) If $D=\ell$ with $\ell \equiv-1 \bmod (25)$, then $t=s_{2}=1, s_{4}=0$, and thus $A=1$, 
$0 \leq I \leq 1, R=0$, whence the types $\alpha_{1}, \alpha_{2}, \alpha_{3}, \beta_{1}, \beta_{2}, \gamma, \delta_{1}, \varepsilon, \zeta_{1}, \eta$ with either $A \geq 2$ or $I=2$ or $R \geq 1$ are excluded, and only the types $\delta_{2}, \zeta_{2}, \vartheta$ remain admissible.

3) If $D=\ell$ with $\ell \equiv+1 \bmod (25)$, then $t=s_{4}=1, s_{2}=0$, and thus $A=1$, $0 \leq I \leq 1, \quad 0 \leq R \leq 2$, whence the types $\alpha_{3}, \beta_{1}, \beta_{2}, \gamma, \varepsilon, \eta$ with either $A \geq 2$ or $I=2$ are excluded, and only the types $\alpha_{1}, \alpha_{2}, \delta_{1}, \delta_{2}, \zeta_{1}, \zeta_{2}, \vartheta$ remain admissible.

4) If $D=q$ with $q \equiv \pm 2 \bmod (5)$ but $q \neq \equiv \pm 7 \bmod (25)$, then $t=2$, $s_{2}=s_{4}=0$, and thus $1 \leq A \leq 2, I=R=0$. These conditions eliminate the types $\alpha_{1}, \alpha_{2}, \alpha_{3}, \beta_{1}, \beta_{2}, \gamma, \delta_{1}, \delta_{2}, \zeta_{1}, \zeta_{2}$ with either $A=3$ or $I \geq 1$ or $R \geq 1$, and only the types $\varepsilon, \eta, \vartheta$ remain admissible. However, the congruence requirement modulo 25 discourages the types $\eta, \vartheta$, and only type $\varepsilon$ is possible.

5) If $D=\ell$ with $\ell \equiv-1 \bmod (5)$ but $\ell \neq \equiv-1 \bmod (25)$, then $t=2, s_{2}=1$, $s_{4}=0$, and thus $1 \leq A \leq 2,0 \leq I \leq 1, R=0$, whence the types $\alpha_{1}, \alpha_{2}, \alpha_{3}, \beta_{1}, \gamma, \delta_{1}, \zeta_{1}$ with either $A=3$ or $I=2$ or $R \geq 1$ are forbidden. The types $\zeta_{2}, \eta, \vartheta$ are excluded by congruence conditions, and only the types $\beta_{2}, \delta_{2}, \varepsilon$ remain admissible.

6) If $D=\ell$ with $\ell \equiv+1 \bmod (5)$ but $\ell \neq \equiv+1 \bmod (25)$ then $t=2, s_{2}=0$, $s_{4}=1$, and thus $1 \leq A \leq 2,0 \leq I \leq 1,0 \leq R \leq 2$, whence the types $\alpha_{3}, \gamma$ with either $A=3$ or $I=2$ are forbidden. The types $\zeta_{1}, \zeta_{2}, \eta, \vartheta$ are excluded by congruence conditions, and only the types $\alpha_{1}, \alpha_{2}, \beta_{1}, \beta_{2}, \delta_{1}, \delta_{2}, \varepsilon$ remain admissible.

Example 4.3 Concerning numerical realizations of Theorem 4.6, we refer to Corollary 4.1 for the parametrized infinite sequences [7] and [2] which realize item (1) and (4). (See also Tables 23 and 19 for the types $\vartheta$ and $\varepsilon$.) In all the other cases, there occurs type splitting:

The similarity class [149] partially realizes item (2). (See Table 38 for the type $\delta_{2}$.) Outside the range of our systematic investigations, we found that the similarity class [1049] realizes type $\zeta_{2}$. Realizations of the type $\vartheta$ are unknown up to now.

The similarity classes [401], [151] and [101] partially realize item (3). (See Table 31, Table 32 and Table 40 for the types $\alpha_{1}, \alpha_{2}$ and $\zeta_{1}$.) Outside the range of our systematic investigations, we found that the similarity class [1151], resp. [3251], realizes type $\delta_{1}$, resp. $\delta_{2}$. Realizations of the types $\zeta_{2}$ and $\vartheta$ are unknown up to now.

The similarity classes [139], [19] and [379] completely realize item (5). (See Table 35, Table 38 and Table 39 for the types $\beta_{2}, \delta_{2}$ and $\varepsilon$.)

The similarity classes [31], [11], [191] and [211] partially realize item (6). (See Table 31, Table 32, Table 34 and Table 37 for the types $\alpha_{1}, \alpha_{2}, \beta_{1}$ and $\delta_{1}$.) Realizations of the types $\beta_{2}, \delta_{2}$ and $\varepsilon$ are unknown up to now.

\subsection{Non-Elementary 5-Class Groups}

Although most of the 5-class groups of pure metacyclic fields $N$, maximal real 
subfields $M$ and pure quintic subfields $L$ are elementary abelian, there occur sparse examples with non-elementary structure. For instance, we have only 8 occurrences within the range $2 \leq D<10^{3}$ of our computations:

1) $\mathrm{Cl}_{5}(N) \simeq C_{25} \times C_{5}^{3},\left(V_{L}, V_{M}, V_{N} ; E\right)=(1,2,5 * ; 6)$ for $D=259=7 \times 37$ (type $\gamma$ ),

2) $\mathrm{Cl}_{5}(N) \simeq C_{25} \times C_{5}^{7}, \mathrm{Cl}_{5}(M) \simeq C_{25} \times C_{5}^{3},\left(V_{L}, V_{M}, V_{N} ; E\right)=(3,5 *, 9 * ; 2)$ for $D=281$ prime (type $\alpha_{1}$ ),

3) $\mathrm{Cl}_{5}(N) \simeq C_{25} \times C_{5}^{5},\left(V_{L}, V_{M}, V_{N} ; E\right)=(2,3,7 * ; 4)$ for $D=465=3 \times 5 \times 31$ (type $\beta_{2}$ ),

4) $\mathrm{Cl}_{5}(N) \simeq C_{25} \times C_{5}^{5},\left(V_{L}, V_{M}, V_{N} ; E\right)=(2,3,7 * ; 4)$ for $D=473=11 \times 43$ (type $\beta_{2}$ ),

5) $\mathrm{Cl}_{5}(N) \simeq C_{25} \times C_{5}^{6}, \mathrm{Cl}_{5}(M) \simeq C_{25} \times C_{5}^{2}, \mathrm{Cl}_{5}(L) \simeq C_{25}$, $\left(V_{L}, V_{M}, V_{N} ; E\right)=(2 *, 4 *, 8 * ; 5)$ for $D=502=2 \times 251$ (type $\left.\beta_{1}\right)$,

6) $\mathrm{Cl}_{5}(N) \simeq C_{25} \times C_{5}^{5},\left(V_{L}, V_{M}, V_{N} ; E\right)=(2,3,7 * ; 4)$ for $D=590=2 \times 5 \times 59$ (type $\beta_{2}$ ),

7) $\mathrm{Cl}_{5}(N) \simeq C_{25}^{2} \times C_{5}^{4}, \mathrm{Cl}_{5}(M) \simeq C_{25} \times C_{5}^{2},\left(V_{L}, V_{M}, V_{N} ; E\right)=(2,4 *, 8 * ; 5)$ for $D=620=2^{2} \times 5 \times 31$ (type $\beta_{1}$ ),

8) $\mathrm{Cl}_{5}(N) \simeq C_{25} \times C_{5}^{4}, \quad \mathrm{Cl}_{5}(M) \simeq C_{25} \times C_{5}, \quad \mathrm{Cl}_{5}(L) \simeq C_{25}$, $\left(V_{L}, V_{M}, V_{N} ; E\right)=(2 *, 3 *, 6 * ; 3)$ for $D=955=5 \times 191$ (type $\left.\alpha_{2}\right)$.

However, outside the range of systematic computations, we additionally found:

a) $\mathrm{Cl}_{5}(N) \simeq C_{25}^{3} \times C_{5}, \quad \mathrm{Cl}_{5}(M) \simeq C_{25} \times C_{5}, \mathrm{Cl}_{5}(L) \simeq C_{25}$, $\left(V_{L}, V_{M}, V_{N} ; E\right)=(2 *, 3 *, 7 * ; 4)$ for $D=1049$ prime (type $\left.\zeta_{2}\right)$,

b) $\mathrm{Cl}_{5}(N) \simeq C_{25}^{2} \times C_{5}^{6}, \quad \mathrm{Cl}_{5}(M) \simeq C_{25} \times C_{5}^{3}, \quad\left(V_{L}, V_{M}, V_{N} ; E\right)=(3,5 *, 10 * ; 3)$ for $D=3001$ prime (type $\alpha_{2}$ ),

c) $\mathrm{Cl}_{5}(N) \simeq C_{25}^{5} \times C_{5}^{4}, \mathrm{Cl}_{5}(M) \simeq C_{25}^{2} \times C_{5}^{3}, \mathrm{Cl}_{5}(L) \simeq C_{25} \times C_{5}^{2}$, $\left(V_{L}, V_{M}, V_{N} ; E\right)=(4 *, 7 *, 14 * ; 3)$ for $D=3251$ prime (type $\left.\delta_{2}\right)$,

d) $\mathrm{Cl}_{5}(N) \simeq C_{25}^{2} \times C_{5}^{2}, \mathrm{Cl}_{5}(M) \simeq C_{25} \times C_{5}, \mathrm{Cl}_{5}(L) \simeq C_{25}$, $\left(V_{L}, V_{M}, V_{N} ; E\right)=(2 *, 3 *, 6 * ; 3)$ for $D=5849$ prime (type $\left.\delta_{2}\right)$.

We point out that in all of the last four examples, the normal field $N$ is a Polya field, since the radicands $D$ are primes $\ell \equiv \pm 1 \bmod (25)$, the conductors are primes $f=\ell$, and thus all primitive ambiguous ideals are principal, generated by the radical $\delta=\sqrt[5]{D}$ and its powers. Consequently, there seems to be no upper bound for the complexity of 5-class groups $\mathrm{Cl}_{5}(N)$ of pure metacyclic Polya fields $N$ in Theorem 4.4 .

\subsection{Refinement of DPF Types by Similarity Classes}

Based on the definition of similarity classes and prototypes in $\S 4.2$, on the explicit listing of all prototypes in the range between 2 and $10^{3}$ in Tables 27-30, and on theoretical foundations in $\S 4.3$, we are now in the position to establish the intended refinement of our 13 differential principal factorization types into similarity classes in Tables $31-43$, as far as the range of our computations for normalized radicands $2 \leq D<10^{3}$ is concerned. The cardinalities $|\mathbf{M}|$ refine the statistical evaluation in Table 26. 
DPF types are characterized by the multiplet $(U, \eta, \zeta ; A, I, R)$, refined Dedekind species, $\mathrm{S}$, by the multiplet $\left(e_{0} ; t, u, v, m ; n, s_{2}, s_{4}\right)$, and 5 -class groups by the multiplet $\left(V_{L}, V_{M}, V_{N} ; E\right)$.

DPF type $\alpha_{1}$ splits into 3 similarity classes in the ground state $\left(V_{L}, V_{M}, V_{N}\right)=(2,3,5)$ and 2 similarity classes in the first excited state $\left(V_{L}, V_{M}, V_{N}\right)=(3,5,9)$. Summing up the partial frequencies $6+3$ of these states in Table 31 yields the modest absolute frequency 9 of type $\alpha_{1}$ in the range $2 \leq D<10^{3}$, as given in Table 26. The logarithmic subfield unit index of type $\alpha_{1}$ is restricted to the single value $E=2$. Type $\alpha_{1}$ is the unique type with 2-dimensional relative principal factorization, $R=2$.

The logarithmic subfield unit index of DPF type $\alpha_{2}$ can take two values, either $E=3$ or $E=1$. Type $\alpha_{2}$ with $E=3$ splits into 5 similarity classes in the ground state $\left(V_{L}, V_{M}, V_{N}\right)=(1,1,2)$ and 6 similarity classes in the first excited state $\left(V_{L}, V_{M}, V_{N}\right)=(2,3,6)$. Type $\alpha_{2}$ with $E=1 \quad$ splits into 7 similarity classes in the ground state $\left(V_{L}, V_{M}, V_{N}\right)=(2,2,4)$ and 4 similarity classes in the first excited state $\left(V_{L}, V_{M}, V_{N}\right)=(3,4,8)$. Summing up the partial frequencies $40+7$, resp. $24+4$, of these states in Table 32 yields the considerable absolute frequency 75 of type $\alpha_{2}$ in the range $2 \leq D<10^{3}$, as given in Table 26. Type $\alpha_{2}$ is the unique type with mixed intermediate and relative principal factorization, $I=R=1$.

DPF type $\alpha_{3}$ splits into 4 similarity classes in the ground state $\left(V_{L}, V_{M}, V_{N}\right)=(2,2,5)$ and 1 similarity class in the first excited state $\left(V_{L}, V_{M}, V_{N}\right)=(3,4,9)$. Summing up the partial frequencies $7+1$ of these states in Table 33 yields the modest absolute frequency 8 of type $\alpha_{3}$ in the range $2 \leq D<10^{3}$, as given in Table 26. The logarithmic subfield unit index of type $\alpha_{3}$ is restricted to the unique value $E=2$. Type $\alpha_{3}$ is the unique type with 2-dimensional intermediate principal factorization, $I=2$.

The logarithmic subfield unit index of DPF type $\beta_{1}$ can take two values, either $E=3$ or $E=5$. Type $\beta_{1}$ with $E=3$ consists of 3 similarity classes in the ground state $\left(V_{L}, V_{M}, V_{N}\right)=(2,3,6)$. Type $\beta_{1}$ with $E=5$ splits into 5 similarity classes in the ground state $\left(V_{L}, V_{M}, V_{N}\right)=(1,2,4)$ and 2 similarity classes in the first excited state $\left(V_{L}, V_{M}, V_{N}\right)=(2,4,8)$. Summing up the partial frequencies 9 , resp. $12+2$, of these states in Table 34 yields the modest absolute frequency 23 of type $\beta_{1}$ in the range $2 \leq D<10^{3}$, as given in Table 26. Type $\beta_{1}$ is the unique type with mixed absolute and relative principal factorization, $A=2$ and $R=1$.

DPF type $\beta_{2}$ splits into 16 similarity classes in the ground state $\left(V_{L}, V_{M}, V_{N}\right)=(1,1,3)$ and 9 similarity classes in the first excited state $\left(V_{L}, V_{M}, V_{N}\right)=(2,3,7)$. Summing up the partial frequencies $146+15$ of these states in Table 35 yields the high absolute frequency 161 of type $\beta_{2}$ in the range $2 \leq D<10^{3}$, as given in Table 26. The logarithmic subfield unit index of type $\beta_{2}$ is restricted to the unique value $E=4$. Type $\beta_{2}$ is the unique type with mixed absolute and intermediate principal factorization, $A=2$ and $I=1$. 
Table 31. Splitting of type $\alpha_{1},(U, \eta, \zeta ; A, I, R)=(2,-,-; 1,0,2): 5$ similarity classes.

\begin{tabular}{cccccccccccccccc}
\hline No. & $\mathrm{S}$ & $e_{0}$ & $t$ & $u$ & $V$ & $m$ & $n$ & $s_{2}$ & $s_{4}$ & $V_{L}$ & $V_{M}$ & $V_{N}$ & $E$ & $\mathbf{M}$ & $|\mathbf{M}|$ \\
\hline 1 & $1 \mathrm{~b}$ & 2 & 1 & 0 & 1 & 1 & 0 & 0 & 1 & 2 & 3 & 5 & 2 & 31 & 2 \\
2 & $1 \mathrm{a}$ & 6 & 1 & 0 & 1 & 4 & 0 & 0 & 1 & 2 & 3 & 5 & 2 & $\mathbf{1 5 5}$ & 2 \\
3 & $1 \mathrm{~b}$ & 2 & 1 & 0 & 1 & 1 & 0 & 0 & 1 & 3 & $5^{*}$ & $9^{*}$ & 2 & $\mathbf{2 8 1}$ & 1 \\
4 & $1 \mathrm{~b}$ & 2 & 2 & 0 & 2 & 3 & 0 & 0 & 2 & 3 & 5 & 9 & 2 & 341 & 2 \\
5 & 2 & 0 & 1 & $1^{\prime}$ & 0 & 1 & 0 & 0 & $1^{\prime}$ & 2 & 3 & 5 & 2 & 401 & 2 \\
\hline
\end{tabular}

Table 32. Splitting of type $\alpha_{2},(U, \eta, \zeta ; A, I, R)=(2,-,-; 1,1,1): 22$ similarity classes.

\begin{tabular}{|c|c|c|c|c|c|c|c|c|c|c|c|c|c|c|c|}
\hline No. & $S$ & $e_{0}$ & $t$ & $u$ & $V$ & $m$ & $n$ & $s_{2}$ & $s_{4}$ & $V_{L}$ & $V_{M}$ & $V_{N}$ & $E$ & $\mathbf{M}$ & $|\mathbf{M}|$ \\
\hline 1 & $1 b$ & 2 & 1 & 0 & 1 & 1 & 0 & 0 & 1 & 1 & 1 & 2 & 3 & 11 & 14 \\
\hline 2 & $1 b$ & 2 & 2 & 0 & 2 & 3 & 1 & 0 & 1 & 2 & 2 & 4 & 1 & 33 & 8 \\
\hline 3 & 1a & 6 & 1 & 0 & 1 & 4 & 0 & 0 & 1 & 1 & 1 & 2 & 3 & 55 & 6 \\
\hline 4 & $1 b$ & 2 & 2 & 0 & 2 & 3 & 1 & 0 & 1 & 1 & 1 & 2 & 3 & 82 & 15 \\
\hline 5 & $1 \mathrm{~b}$ & 2 & 2 & 0 & 2 & 3 & 1 & 0 & 1 & 2 & 3 & 6 & 3 & 123 & 2 \\
\hline 6 & $1 b$ & 2 & 1 & 0 & 1 & 1 & 0 & 0 & 1 & 2 & 2 & 4 & 1 & 131 & 6 \\
\hline 7 & 2 & 0 & 1 & $1^{\prime}$ & 0 & 1 & 0 & 0 & $1^{\prime}$ & 1 & 1 & 2 & 3 & 151 & 3 \\
\hline 8 & 1a & 6 & 1 & 0 & 1 & 4 & 0 & 0 & 1 & 2 & 2 & 4 & 1 & 275 & 2 \\
\hline 9 & $1 \mathrm{~b}$ & 2 & 2 & 1 & 1 & 4 & 1 & 0 & 1 & 2 & 2 & 4 & 1 & 287 & 1 \\
\hline 10 & 2 & 0 & 3 & 0 & 3 & 3 & 1 & 1 & 1 & 2 & 3 & 6 & 3 & 418 & 1 \\
\hline 11 & 2 & 0 & 2 & 0 & 2 & 1 & 0 & 0 & 2 & 2 & 3 & 6 & 3 & 451 & 1 \\
\hline 12 & $1 \mathrm{a}$ & 6 & 2 & 0 & 2 & 16 & 1 & 0 & 1 & 2 & 3 & 6 & 3 & 550 & 1 \\
\hline 13 & $1 \mathrm{~b}$ & 2 & 2 & 0 & 2 & 3 & 0 & 0 & 2 & 3 & 4 & 8 & 1 & 671 & 1 \\
\hline 14 & 2 & 0 & 3 & 0 & 3 & 3 & 1 & 0 & 2 & 2 & 3 & 6 & 3 & 682 & 1 \\
\hline 15 & $1 \mathrm{~b}$ & 2 & 1 & 0 & 1 & 1 & 0 & 0 & 1 & 3 & 4 & 8 & 1 & 691 & 1 \\
\hline 16 & 1a & 6 & 2 & 0 & 2 & 16 & 1 & 0 & 1 & 2 & 2 & 4 & 1 & 710 & 4 \\
\hline 17 & 2 & 0 & 1 & $1^{\prime}$ & 0 & 1 & 0 & 0 & $1^{\prime}$ & 2 & 2 & 4 & 1 & 751 & 1 \\
\hline 18 & $1 b$ & 2 & 2 & 0 & 2 & 3 & 0 & 1 & 1 & 3 & 4 & 8 & 1 & 779 & 1 \\
\hline 19 & $1 b$ & 2 & 2 & $1^{\prime}$ & 1 & 4 & 1 & 0 & $1^{\prime}$ & 2 & 2 & 4 & 1 & 808 & 2 \\
\hline 20 & $1 b$ & 2 & 3 & 1 & 2 & 12 & 2 & 0 & 1 & 3 & 4 & 8 & 1 & 861 & 1 \\
\hline 21 & la & 6 & 1 & 0 & 1 & 4 & 0 & 0 & 1 & $2^{*}$ & $3^{*}$ & $6^{*}$ & 3 & 955 & 1 \\
\hline 22 & 2 & 0 & 2 & 0 & 2 & 1 & 1 & 0 & 1 & 1 & 1 & 2 & 3 & 982 & 2 \\
\hline
\end{tabular}

Table 33. Splitting of type $\alpha_{3},(U, \eta, \zeta ; A, I, R)=(2,-,-; 1,2,0): 5$ similarity classes.

\begin{tabular}{cccccccccccccccc}
\hline No. & $\mathrm{S}$ & $e_{0}$ & $t$ & $u$ & $V$ & $m$ & $n$ & $s_{2}$ & $s_{4}$ & $V_{L}$ & $V_{M}$ & $V_{N}$ & $E$ & $\mathbf{M}$ & $|\mathbf{M}|$ \\
\hline 1 & $1 \mathrm{~b}$ & 2 & 2 & 0 & 2 & 3 & 0 & 1 & 1 & 2 & 2 & 5 & 2 & 319 & 3 \\
2 & 2 & 0 & 2 & 0 & 2 & 1 & 0 & 2 & 0 & 2 & 2 & 5 & 2 & 551 & 1 \\
3 & $1 \mathrm{~b}$ & 2 & 3 & 0 & 3 & 13 & 1 & 1 & 1 & 3 & 4 & 9 & 2 & 627 & 1 \\
4 & 2 & 0 & 2 & 0 & 2 & 1 & 0 & 1 & 1 & 2 & 2 & 5 & 2 & 649 & 2 \\
5 & 2 & 0 & 3 & 0 & 3 & 3 & 1 & 1 & 1 & 2 & 2 & 5 & 2 & 957 & 1 \\
\hline
\end{tabular}


Table 34. Splitting of type $\beta_{1},(U, \eta, \zeta ; A, I, R)=(2,-,-; 2,0,1): 10$ similarity classes.

\begin{tabular}{cccccccccccccccc}
\hline No. & $\mathrm{S}$ & $e_{0}$ & $t$ & $u$ & $V$ & $m$ & $n$ & $s_{2}$ & $s_{4}$ & $V_{L}$ & $V_{M}$ & $V_{N}$ & $E$ & $\mathbf{M}$ & $|\mathbf{M}|$ \\
\hline 1 & $1 \mathrm{~b}$ & 2 & 3 & 0 & 3 & 13 & 2 & 0 & 1 & 2 & 3 & 6 & 3 & $\mathbf{1 8 6}$ & 7 \\
2 & $1 \mathrm{~b}$ & 2 & 1 & 0 & 1 & 1 & 0 & 0 & 1 & 1 & 2 & 4 & 5 & $\mathbf{1 9 1}$ & 3 \\
3 & $1 \mathrm{~b}$ & 2 & 2 & 0 & 2 & 3 & 1 & 0 & 1 & 1 & 2 & 4 & 5 & $\mathbf{2 5 3}$ & 4 \\
4 & $1 \mathrm{~b}$ & 2 & 2 & $1^{\prime}$ & 1 & 4 & 1 & 0 & $1^{\prime}$ & 1 & 2 & 4 & 5 & $\mathbf{3 0 2}$ & 2 \\
5 & 2 & 0 & 2 & 0 & 2 & 1 & 1 & 0 & 1 & 1 & 2 & 4 & 5 & $\mathbf{4 8 2}$ & 2 \\
6 & $1 \mathrm{~b}$ & 2 & 2 & $1^{\prime}$ & 1 & 4 & 1 & 0 & $1^{\prime}$ & $2^{*}$ & $4^{*}$ & $8^{*}$ & 5 & $\mathbf{5 0 2}$ & 1 \\
7 & $1 \mathrm{~b}$ & 2 & 2 & 0 & 2 & 3 & 1 & 0 & 1 & 2 & 3 & 6 & 3 & 517 & 1 \\
8 & $1 \mathrm{a}$ & 6 & 2 & 0 & 2 & 16 & 1 & 0 & 1 & 2 & $4^{*}$ & $8^{*}$ & 5 & $\mathbf{6 2 0}$ & 1 \\
9 & 2 & 0 & 3 & 1 & 2 & 4 & 2 & 0 & 1 & 2 & 3 & 6 & 3 & $\mathbf{6 9 3}$ & 1 \\
10 & $1 \mathrm{a}$ & 6 & 2 & 0 & 2 & 16 & 1 & 0 & 1 & 1 & 2 & 4 & 5 & $\mathbf{8 2 5}$ & 1 \\
\hline
\end{tabular}

Table 35. Splitting of type $\beta_{2},(U, \eta, \zeta ; A, I, R)=(2,-,-; 2,1,0): 25$ similarity classes.

\begin{tabular}{|c|c|c|c|c|c|c|c|c|c|c|c|c|c|c|c|}
\hline No. & S & $e_{0}$ & $t$ & $u$ & $V$ & $m$ & $n$ & $s_{2}$ & $s_{4}$ & $V_{L}$ & $V_{M}$ & $V_{N}$ & $E$ & $\mathbf{M}$ & $|\mathbf{M}|$ \\
\hline 1 & $1 b$ & 2 & 2 & 0 & 2 & 3 & 1 & 0 & 1 & 1 & 1 & 3 & 4 & 22 & 35 \\
\hline 2 & $1 b$ & 2 & 2 & 0 & 2 & 3 & 1 & 1 & 0 & 1 & 1 & 3 & 4 & 38 & 44 \\
\hline 3 & $1 b$ & 2 & 2 & 1 & 1 & 4 & 1 & 0 & 1 & 1 & 1 & 3 & 4 & 77 & 7 \\
\hline 4 & 1a & 6 & 2 & 0 & 2 & 16 & 1 & 0 & 1 & 1 & 1 & 3 & 4 & 110 & 11 \\
\hline 5 & 2 & 0 & 3 & 0 & 3 & 3 & 2 & 0 & 1 & 1 & 1 & 3 & 4 & 132 & 5 \\
\hline 6 & $1 b$ & 2 & 2 & 1 & 1 & 4 & 1 & 1 & 0 & 1 & 1 & 3 & 4 & 133 & 7 \\
\hline 7 & $1 b$ & 2 & 1 & 0 & 1 & 1 & 0 & 1 & 0 & 1 & 1 & 3 & 4 & 139 & 4 \\
\hline 8 & $1 b$ & 2 & 3 & 1 & 2 & 12 & 2 & 0 & 1 & 2 & 3 & 7 & 4 & 154 & 3 \\
\hline 9 & 2 & 0 & 3 & 0 & 3 & 3 & 2 & 1 & 0 & 1 & 1 & 3 & 4 & 174 & 3 \\
\hline 10 & $1 \mathrm{a}$ & 6 & 2 & 0 & 2 & 16 & 1 & 1 & 0 & 1 & 1 & 3 & 4 & 190 & 9 \\
\hline 11 & $1 b$ & 2 & 2 & $1^{\prime}$ & 1 & 4 & 1 & 0 & $1^{\prime}$ & 1 & 1 & 3 & 4 & 202 & 6 \\
\hline 12 & $1 \mathrm{~b}$ & 2 & 2 & 0 & 2 & 3 & 0 & 1 & 1 & 2 & 3 & 7 & 4 & 209 & 2 \\
\hline 13 & $1 \mathrm{~b}$ & 2 & 3 & 0 & 3 & 13 & 2 & 0 & 1 & 2 & 3 & 7 & 4 & 286 & 3 \\
\hline 14 & $1 \mathrm{a}$ & 6 & 2 & 1 & 1 & 16 & 1 & 0 & 1 & 1 & 1 & 3 & 4 & 385 & 1 \\
\hline 15 & $1 b$ & 2 & 2 & $1^{\prime}$ & 1 & 4 & 1 & $1^{\prime}$ & 0 & 1 & 1 & 3 & 4 & 398 & 7 \\
\hline 16 & 2 & 0 & 3 & 1 & 2 & 4 & 2 & 1 & 0 & 1 & 1 & 3 & 4 & 399 & 2 \\
\hline 17 & $1 \mathrm{a}$ & 6 & 2 & 0 & 2 & 16 & 1 & 0 & 1 & 2 & 3 & $7^{*}$ & 4 & 465 & 1 \\
\hline 18 & $1 b$ & 2 & 2 & 1 & 1 & 4 & 1 & 0 & 1 & 2 & 3 & $7^{*}$ & 4 & 473 & 1 \\
\hline 19 & 2 & 0 & 3 & 1 & 2 & 4 & 2 & 0 & 1 & 1 & 1 & 3 & 4 & 574 & 3 \\
\hline 20 & 1a & 6 & 2 & 0 & 2 & 16 & 1 & 1 & 0 & 2 & 3 & $7^{*}$ & 4 & 590 & 1 \\
\hline 21 & $1 b$ & 2 & 3 & 1 & 2 & 12 & 2 & 1 & 0 & 2 & 3 & 7 & 4 & 609 & 1 \\
\hline 22 & $1 b$ & 2 & 3 & 0 & 3 & 13 & 1 & 1 & 1 & 2 & 3 & 7 & 4 & 638 & 2 \\
\hline 23 & 1a & 6 & 2 & 1 & 1 & 16 & 1 & 1 & 0 & 1 & 1 & 3 & 4 & 665 & 1 \\
\hline 24 & 2 & 0 & 2 & 0 & 2 & 1 & 1 & 1 & 0 & 1 & 1 & 3 & 4 & 893 & 1 \\
\hline 25 & $1 \mathrm{~b}$ & 2 & 3 & 0 & 3 & 1. & 1 & 0 & 2 & 2 & 3 & 7 & 4 & 902 & 1 \\
\hline
\end{tabular}


Table 36. Splitting of type $\gamma,(U, \eta, \zeta ; A, I, R)=(2,-,-; 3,0,0): 29$ similarity classes.

\begin{tabular}{|c|c|c|c|c|c|c|c|c|c|c|c|c|c|c|c|}
\hline No. & $S$ & $e_{0}$ & $t$ & $u$ & $V$ & $m$ & $n$ & $s_{2}$ & $s_{4}$ & $V_{L}$ & $V_{M}$ & $V_{N}$ & $E$ & $\mathbf{M}$ & $|\mathbf{M}|$ \\
\hline 1 & $1 b$ & 2 & 2 & 0 & 2 & 3 & 2 & 0 & 0 & 0 & 0 & 1 & 6 & 6 & 77 \\
\hline 2 & $1 \mathrm{~b}$ & 2 & 2 & 1 & 1 & 4 & 2 & 0 & 0 & 0 & 0 & 1 & 6 & 14 & 44 \\
\hline 3 & 1a & 6 & 2 & 0 & 2 & 16 & 2 & 0 & 0 & 0 & 0 & 1 & 6 & 30 & 37 \\
\hline 4 & $1 b$ & 2 & 3 & 1 & 2 & 12 & 3 & 0 & 0 & 1 & 2 & 5 & 6 & 42 & 22 \\
\hline 5 & $1 \mathrm{~b}$ & 2 & 3 & 0 & 3 & 13 & 2 & 0 & 1 & 1 & 2 & 5 & 6 & 66 & 17 \\
\hline 6 & 1a & 6 & 2 & 1 & 1 & 16 & 2 & 0 & 0 & 0 & 0 & 1 & 6 & 70 & 14 \\
\hline 7 & $1 \mathrm{~b}$ & 2 & 3 & 0 & 3 & 13 & 3 & 0 & 0 & 1 & 2 & 5 & 6 & 78 & 37 \\
\hline 8 & $1 b$ & 2 & 3 & 0 & 3 & 13 & 2 & 1 & 0 & 1 & 2 & 5 & 6 & 114 & 20 \\
\hline 9 & 2 & 0 & 3 & 1 & 2 & 4 & 3 & 0 & 0 & 0 & 0 & 1 & 6 & 126 & 6 \\
\hline 10 & $1 \mathrm{a}$ & 6 & 3 & 1 & 2 & 64 & 3 & 0 & 0 & 1 & 2 & 5 & 6 & 210 & 5 \\
\hline 11 & $1 \mathrm{~b}$ & 2 & 3 & 1 & 2 & 12 & 2 & 0 & 1 & 1 & 2 & 5 & 6 & 231 & 5 \\
\hline 12 & $1 b$ & 2 & 2 & 0 & 2 & 3 & 1 & 1 & 0 & 1 & 2 & 5 & 6 & 247 & 2 \\
\hline 13 & $1 \mathrm{~b}$ & 2 & 2 & 1 & 1 & 4 & 2 & 0 & 0 & 1 & 2 & 5 & 6 & 259 & 1 \\
\hline 14 & $1 \mathrm{~b}$ & 2 & 3 & 1 & 2 & 12 & 2 & 1 & 0 & 1 & 2 & 5 & 6 & 266 & 3 \\
\hline 15 & 2 & 0 & 3 & 0 & 3 & 3 & 3 & 0 & 0 & 0 & 0 & 1 & 6 & 276 & 10 \\
\hline 16 & $1 \mathrm{a}$ & 6 & 2 & 0 & 2 & 16 & 1 & 1 & 0 & 1 & 2 & 5 & 6 & 285 & 1 \\
\hline 17 & 1a & 6 & 3 & 0 & 3 & 64 & 2 & 0 & 1 & 2 & 4 & 9 & 6 & 330 & 2 \\
\hline 18 & $1 \mathrm{a}$ & 6 & 3 & 0 & 3 & 64 & 3 & 0 & 0 & 1 & 2 & 5 & 6 & 390 & 4 \\
\hline 19 & $1 \mathrm{~b}$ & 2 & 4 & 1 & 3 & 52 & 3 & 0 & 1 & 2 & 4 & 9 & 6 & 462 & 1 \\
\hline 20 & $1 \mathrm{~b}$ & 2 & 4 & 1 & 3 & 25 & 4 & 0 & 0 & 2 & 4 & 9 & 6 & 546 & 3 \\
\hline 21 & 1a & 6 & 3 & 0 & 3 & 64 & 2 & 1 & 0 & 1 & 2 & 5 & 6 & 570 & 2 \\
\hline 22 & $1 b$ & 2 & 3 & 2 & 1 & 16 & 3 & 0 & 0 & 1 & 2 & 5 & 6 & 602 & 2 \\
\hline 23 & $1 \mathrm{~b}$ & 2 & 3 & $1^{\prime}$ & 2 & 12 & 2 & 0 & $1^{\prime}$ & 1 & 2 & 5 & 6 & 606 & 2 \\
\hline 24 & $1 \mathrm{a}$ & 6 & 3 & 0 & 3 & 64 & 2 & 0 & 1 & 1 & 2 & 5 & 6 & 660 & 2 \\
\hline 25 & $1 \mathrm{a}$ & 6 & 3 & 1 & 2 & 64 & 2 & 0 & 1 & 1 & 2 & 5 & 6 & 770 & 1 \\
\hline 26 & $1 b$ & 2 & 4 & 1 & 3 & 52 & 3 & 1 & 0 & 2 & 4 & 9 & 6 & 798 & 1 \\
\hline 27 & $1 \mathrm{~b}$ & 2 & 4 & 0 & 4 & 51 & 3 & 0 & 1 & 2 & 4 & 9 & 6 & 858 & 1 \\
\hline 28 & $1 \mathrm{~b}$ & 2 & 3 & $1^{\prime}$ & 2 & 12 & 2 & $1^{\prime}$ & 0 & 1 & 2 & 5 & 6 & 894 & 1 \\
\hline 29 & 2 & 0 & 4 & 1 & 3 & 12 & 3 & 0 & 1 & 1 & 2 & 5 & 6 & 924 & 1 \\
\hline
\end{tabular}

DPF type $\gamma$ splits into 6 similarity classes in the ground state $\left(V_{L}, V_{M}, V_{N}\right)=(0,0,1), 16$ similarity classes in the first excited state $\left(V_{L}, V_{M}, V_{N}\right)=(1,2,5)$, and 5 similarity classes in the second excited state $\left(V_{L}, V_{M}, V_{N}\right)=(2,4,9)$. Summing up the partial frequencies $188+128+8$ of these states in Table 36 yields the maximal absolute frequency 324 of type $\gamma$ among all 13 types in the range $2 \leq D<10^{3}$, as given in Table 26 . The logarithmic subfield subfield unit index of type $\gamma$ is restricted to the unique value $E=6$. Type $\gamma$ is the unique type with 3-dimensional absolute principal factorization, $A=3$. However, the 1-dimensional subspace $\Delta$ is formed by radicals, and only the complementary 2-dimensional subspace is non-trivial.

The logarithmic subfield unit index of DPF type $\delta_{1}$ can take two values, either $E=4$ or $E=2$. Type $\delta_{1}$ with $E=4$ splits into 2 similarity classes in the ground state $\left(V_{L}, V_{M}, V_{N}\right)=(1,2,3)$. Type $\delta_{1}$ with $E=2$ consists of 1 
similarity class in the ground state $\left(V_{L}, V_{M}, V_{N}\right)=(3,5,9)$. Summing up the partial frequencies $6+1$ of these states in Table 37 yields the modest absolute frequency 7 of type $\delta_{1}$ in the range $2 \leq D<10^{3}$, as given in Table 26. Type $\delta_{1}$ is a type with 1-dimensional relative principal factorization, $R=1$.

DPF type $\delta_{2}$ splits into 4 similarity classes in the ground state $\left(V_{L}, V_{M}, V_{N}\right)=(1,1,2)$ and 1 similarity class in the first excited state $\left(V_{L}, V_{M}, V_{N}\right)=(2,3,6)$. Summing up the partial frequencies $52+1$ of these states in Table 38 yields the considerable absolute frequency 53 of type $\delta_{2}$ in the range $2 \leq D<10^{3}$, as given in Table 26. The logarithmic subfield unit index of type $\delta_{2}$ is restricted to the unique value $E=3$. Type $\delta_{2}$ is a type with 1-dimensional intermediate principal factorization, $I=1$.

DPF type $\varepsilon$ splits into 3 similarity classes in the ground state $\left(V_{L}, V_{M}, V_{N}\right)=(0,0,0), 16$ similarity classes in the first excited state $\left(V_{L}, V_{M}, V_{N}\right)=(1,2,4)$, and 3 similarity classes in the second excited state $\left(V_{L}, V_{M}, V_{N}\right)=(2,4,8)$. Summing up the partial frequencies $139+61+8$ of these states in Table 39 yields the high absolute frequency 208 of type $\varepsilon$ in the range $2 \leq D<10^{3}$, as given in Table 26. The logarithmic subfield unit index of type $\varepsilon$ is restricted to the unique value $E=5$. Type $\varepsilon$ is a type with 2-dimensional absolute principal factorization, $A=2$.

The logarithmic subfield unit index of DPF type $\zeta_{1}$ is restricted to the unique value $E=5$. Type $\zeta_{1}$ consists of 1 similarity class in the ground state $\left(V_{L}, V_{M}, V_{N}\right)=(1,2,4)$. The frequency 1 of this state in Table 40 coincides with the negligible absolute frequency 1 of type $\zeta_{1}$ in the range $2 \leq D<10^{3}$, as given in Table 26. Type $\zeta_{1}$ is a type with 1 -dimensional relative principal factorization, $R=1$.

DPF type $\zeta_{2}$ consists of 3 similarity classes. The modest absolute frequency 5 of type $\zeta_{2}$ in the range $2 \leq D<10^{3}$, given in Table 26 , is the sum $2+1+2$ of partial frequencies in Table 41. Type $\zeta_{2}$ only occurs with logarithmic subfield unit index $E=4$. It is a type with 1-dimensional intermediate principal factorization, $I=1$.

DPF type $\eta$ splits in 2 similarity classes, [35] and [301]. The modest absolute frequency 7 of type $\eta$ in the range $2 \leq D<10^{3}$, given in Table 26, is the sum $6+1$ of partial frequencies in Table 42. Type $\eta$ only occurs with logarithmic subfield unit index $E=6$. It is a type with 2-dimensional absolute principal factorization, $A=2$. However, it should be pointed out that outside of the range of our systematic investigations we found an excited state $\left(V_{L}, V_{M}, V_{N}\right)=(1,2,5)$ for the similarity class [1505], where $1505=5 \times 7 \times 43$ has three prime divisors, additionally to the ground state $\left(V_{L}, V_{M}, V_{N}\right)=(0,0,1)$.

Table 37. Splitting of type $\delta_{1}, \quad(U, \eta, \zeta ; A, I, R)=(1, \times,-; 1,0,1): 3$ similarity classes.

\begin{tabular}{cccccccccccccccc}
\hline No. & $\mathrm{S}$ & $e_{0}$ & $t$ & $u$ & $V$ & $m$ & $n$ & $s_{2}$ & $s_{4}$ & $V_{L}$ & $V_{M}$ & $V_{N}$ & $E$ & $\mathbf{M}$ & $|\mathbf{M}|$ \\
\hline 1 & $1 \mathrm{~b}$ & 2 & 1 & 0 & 1 & 1 & 0 & 0 & 1 & 3 & 5 & 9 & 2 & $\mathbf{2 1 1}$ & 1 \\
2 & $1 \mathrm{~b}$ & 2 & 1 & 0 & 1 & 1 & 0 & 0 & 1 & 1 & 2 & 3 & 4 & $\mathbf{4 2 1}$ & 5 \\
3 & 2 & 0 & 2 & 0 & 2 & 1 & 1 & 0 & 1 & 1 & 2 & 3 & 4 & $\mathbf{8 4 3}$ & 1 \\
\hline
\end{tabular}


Table 38. Splitting of type $\delta_{2},(U, \eta, \zeta ; A, I, R)=(1, \times,-; 1,1,0): 5$ similarity classes.

\begin{tabular}{cccccccccccccccc}
\hline No. & $\mathrm{S}$ & $e_{0}$ & $t$ & $u$ & $V$ & $m$ & $n$ & $s_{2}$ & $s_{4}$ & $V_{L}$ & $V_{M}$ & $V_{N}$ & $E$ & $\mathbf{M}$ & $|\mathrm{M}|$ \\
\hline 1 & $1 \mathrm{~b}$ & 2 & 1 & 0 & 1 & 1 & 0 & 1 & 0 & 1 & 1 & 2 & 3 & 19 & 27 \\
2 & 2 & 0 & 2 & 0 & 2 & 1 & 1 & 1 & 0 & 1 & 1 & 2 & 3 & 57 & 10 \\
3 & $1 \mathrm{a}$ & 6 & 1 & 0 & 1 & 4 & 0 & 1 & 0 & 1 & 1 & 2 & 3 & 95 & 9 \\
4 & 2 & 0 & 1 & $1^{\prime}$ & 0 & 1 & 0 & 1 & 0 & 1 & 1 & 2 & 3 & 149 & 6 \\
5 & $1 \mathrm{~b}$ & 2 & 2 & 0 & 2 & 3 & 1 & 1 & 0 & 2 & 3 & 6 & 3 & 377 & 1 \\
\hline
\end{tabular}

Table 39. Splitting of type $\varepsilon,(U, \eta, \zeta ; A, I, R)=(1, \times,-; 2,0,0): 22$ similarity classes.

\begin{tabular}{|c|c|c|c|c|c|c|c|c|c|c|c|c|c|c|c|}
\hline No. & $S$ & $e_{0}$ & $t$ & $u$ & $V$ & $m$ & $n$ & $s_{2}$ & $s_{4}$ & $V_{L}$ & $V_{M}$ & $V_{N}$ & $E$ & $\mathbf{M}$ & $|\mathbf{M}|$ \\
\hline 1 & $1 b$ & 2 & 1 & 0 & 1 & 1 & 1 & 0 & 0 & 0 & 0 & 0 & 5 & 2 & 71 \\
\hline 2 & 1a & 6 & 1 & 0 & 1 & 1 & 1 & 0 & 0 & 0 & 0 & 0 & 5 & 10 & 31 \\
\hline 3 & 2 & 0 & 2 & 0 & 2 & 1 & 2 & 0 & 0 & 0 & 0 & 0 & 5 & 18 & 37 \\
\hline 4 & $1 \mathrm{a}$ & 6 & 2 & 1 & 1 & 16 & 2 & 0 & 0 & 1 & 2 & 4 & 5 & 140 & 5 \\
\hline 5 & $1 b$ & 2 & 2 & 0 & 2 & 3 & 2 & 0 & 0 & 1 & 2 & 4 & 5 & 141 & 19 \\
\hline 6 & $1 \mathrm{~b}$ & 2 & 2 & 0 & 2 & 3 & 1 & 1 & 0 & 1 & 2 & 4 & 5 & 171 & 6 \\
\hline 7 & 1a & 6 & 2 & 0 & 2 & 16 & 2 & 0 & 0 & 1 & 2 & 4 & 5 & 180 & 5 \\
\hline 8 & 2 & 0 & 3 & 1 & 2 & 4 & 2 & 0 & 0 & 1 & 2 & 4 & 5 & 182 & 1 \\
\hline 9 & $1 \mathrm{~b}$ & 2 & 2 & 1 & 1 & 4 & 1 & 1 & 0 & 1 & 2 & 4 & 5 & 203 & 2 \\
\hline 10 & 2 & 0 & 2 & 0 & 2 & 1 & 1 & 1 & 0 & 1 & 2 & 4 & 5 & 218 & 1 \\
\hline 11 & $1 \mathrm{~b}$ & 2 & 3 & 1 & 2 & 12 & 3 & 0 & 0 & 2 & 4 & 8 & 5 & 273 & 4 \\
\hline 12 & 1a & 6 & 2 & 0 & 2 & 16 & 1 & 1 & 0 & 1 & 2 & 4 & 5 & 290 & 2 \\
\hline 13 & $1 b$ & 2 & 2 & 0 & 2 & 3 & 1 & 1 & 0 & 1 & 2 & 4 & 5 & 298 & 2 \\
\hline 14 & $1 \mathrm{~b}$ & 2 & 2 & 1 & 1 & 4 & 2 & 0 & 0 & 1 & 2 & 4 & 5 & 329 & 7 \\
\hline 15 & $1 b$ & 2 & 3 & 0 & 3 & 13 & 2 & 1 & 0 & 2 & 4 & 8 & 5 & 348 & 2 \\
\hline 16 & $1 b$ & 2 & 1 & 0 & 1 & 1 & 0 & 1 & 0 & 1 & 2 & 4 & 5 & 379 & 1 \\
\hline 17 & $1 b$ & 2 & 2 & 0 & 2 & 3 & 1 & 0 & 1 & 1 & 2 & 4 & 5 & 422 & 6 \\
\hline 18 & 2 & 0 & 3 & 1 & 2 & 4 & 2 & 1 & 0 & 1 & 2 & 4 & 5 & 532 & 1 \\
\hline 19 & 1a & 6 & 1 & 0 & 1 & 4 & 0 & 1 & 0 & 1 & 2 & 4 & 5 & 695 & 1 \\
\hline 20 & $1 b$ & 2 & 3 & 0 & 3 & 13 & 3 & 0 & 0 & 2 & 4 & 8 & 5 & 702 & 2 \\
\hline 21 & 2 & 0 & 2 & 2 & 0 & 4 & 2 & 0 & 0 & 1 & 2 & 4 & 5 & 749 & 1 \\
\hline 22 & 1a & 6 & 1 & 1 & 0 & 4 & 1 & 0 & 0 & 1 & 2 & 4 & 5 & 785 & 1 \\
\hline
\end{tabular}

Table 40. No splitting of type $\zeta_{1},(U, \eta, \zeta ; A, I, R)=(1,-, \times ; 1,0,1): 1$ similarity class.

\begin{tabular}{cccccccccccccccc}
\hline No. & $\mathrm{S}$ & $e_{0}$ & $t$ & $u$ & $V$ & $m$ & $n$ & $s_{2}$ & $s_{4}$ & $V_{L}$ & $V_{M}$ & $V_{N}$ & $E$ & $\mathbf{M}$ & $|\mathbf{M}|$ \\
\hline 1 & 2 & 0 & 1 & $1^{\prime}$ & 0 & 1 & 0 & 0 & $1^{\prime}$ & 1 & 2 & 4 & 5 & 101 & 1 \\
\hline
\end{tabular}


Table 41. Splitting of type $\zeta_{2},(U, \eta, \zeta ; A, I, R)=(1,-, \times ; 1,1,0): 3$ similarity classes.

\begin{tabular}{cccccccccccccccc}
\hline No. & $S$ & $e_{0}$ & $t$ & $u$ & $V$ & $m$ & $n$ & $s_{2}$ & $s_{4}$ & $V_{L}$ & $V_{M}$ & $V_{N}$ & $E$ & $\mathbf{M}$ & $|\mathbf{M}|$ \\
\hline 1 & $1 \mathrm{a}$ & 6 & 1 & $1^{\prime}$ & 0 & 4 & 0 & 0 & $1^{\prime}$ & 1 & 1 & 3 & 4 & 505 & 2 \\
2 & 2 & 0 & 2 & $2^{\prime}$ & 0 & 4 & 1 & 0 & $1^{\prime}$ & 1 & 1 & 3 & 4 & 707 & 1 \\
3 & $1 \mathrm{a}$ & 6 & 1 & 1 & 0 & 4 & 0 & 1 & 0 & 1 & 1 & 3 & 4 & $\mathbf{7 4 5}$ & 2 \\
\hline
\end{tabular}

Table 42. Splitting of type $\eta,(U, \eta, \zeta ; A, I, R)=(1,-, \times ; 2,0,0): 2$ similarity classes.

\begin{tabular}{cccccccccccccccc}
\hline No. & $\mathrm{S}$ & $e_{0}$ & $t$ & $u$ & $V$ & $m$ & $n$ & $s_{2}$ & $s_{4}$ & $V_{L}$ & $V_{M}$ & $V_{N}$ & $E$ & $\mathbf{M}$ & $|\mathbf{M}|$ \\
\hline 1 & $1 \mathrm{a}$ & 6 & 1 & 1 & 0 & 4 & 1 & 0 & 0 & 0 & 0 & 1 & 6 & 35 & 6 \\
2 & 2 & 0 & 2 & 2 & 0 & 4 & 2 & 0 & 0 & 0 & 0 & 1 & 6 & $\mathbf{3 0 1}$ & 1 \\
\hline
\end{tabular}

DPF type $\vartheta$ splits into the unique finite similarity class [5] with only a single element and the infinite parametrized sequence [7] consisting of all prime radicands $D=q$ congruent to $\pm 7 \bmod (25)$. The small absolute frequency 19 of type $\vartheta$ in the range $2 \leq D<10^{3}$, given in Table 26, is the sum $|\mathbf{5}|+|7|=1+18$ in Table 43. Since no theoretical argument disables the occurrence of type $\vartheta$ for composite radicands $D$ with prime factors 5 and $q \equiv \pm 7 \bmod (25)$, we conjecture that such cases will appear in bigger ranges with $D>10^{3}$. Type $\vartheta$ only occurs with logarithmic subfield unit index $E=5$, and is the unique type where every unit of $K$ occurs as norm of a unit of $N$, that is $U=0$.

\subsection{Increasing Dominance of DPF Type $\gamma$ for $T \rightarrow \infty$}

In this final section, we want to show that the careful book keeping of similarity classes with representative prototypes in Tables $31-43$ is useful for the quantitative illumination of many other phenomena. For an explanation, we select the phenomenon of absolute principal factorizations.

The statistical distribution of DPF types in Table 26 has proved that type $\gamma$ with 324 occurrences, that is $36 \%$, among all 900 fields $N=\mathbb{Q}\left(\zeta_{5}, \sqrt[5]{D}\right)$ with normalized radicands in the range $2 \leq D<10^{3}$ is doubtlessly the high champion of all DPF types. This means that there is a clear trend towards the maximal possible extent of 3-dimensional spaces of absolute principal factorizations, $A=3$, in spite of the disadvantage that the estimate $1 \leq A \leq \min (3, T)$ in the formulas (4.3) and (4.4) of ([6] Cor. 4.1) prohibits type $\gamma$ for conductors $f$ with $T \leq 2$ prime divisors.

For the following investigation, we have to recall that the number $T$ of all prime factors of $f^{4}=5^{e_{0}} \cdot q_{1}^{4} \cdots q_{t}^{4}$ is given by $T=t+1$ for fields of Dedekind's species 1 , where $e_{0} \in\{2,6\}$, and by $T=t$ for fields of Dedekind's species 2, where $e_{0}=0$.

Conductors $f$ with $T=4$ prime factors occur in six tables, 
Table 43. Splitting of type $\vartheta, \quad(U, \eta, \zeta ; A, I, R)=(0, \times, \times ; 1,0,0): 2$ similarity classes.

\begin{tabular}{cccccccccccccccc}
\hline No. & $\mathrm{S}$ & $e_{0}$ & $t$ & $u$ & $V$ & $m$ & $n$ & $s_{2}$ & $s_{4}$ & $V_{L}$ & $V_{M}$ & $V_{N}$ & $E$ & $\mathbf{M}$ & $|\mathbf{M}|$ \\
\hline 1 & $1 \mathrm{a}$ & 6 & 0 & 0 & 0 & 1 & 0 & 0 & 0 & 0 & 0 & 0 & 5 & $\mathbf{5}$ & 1 \\
2 & 2 & 0 & 1 & 1 & 0 & 1 & 1 & 0 & 0 & 0 & 0 & 0 & 5 & 7 & 18 \\
\hline
\end{tabular}

1 case of type $\alpha_{2}$ in a single similarity class of Table 32,

1 case of type $\alpha_{3}$ in a single similarity class of Table 33,

7 cases of type $\beta_{1}$ in a single similarity class of Table 34,

10 cases of type $\beta_{2}$ in 5 similarity classes of Table 35 ,

126 cases of type $\gamma$ in 16 similarity classes of Table 36,

8 cases of type $\varepsilon$ in 3 similarity classes of Table 39,

that is, a total of 153 cases, with respect to the complete range $2 \leq D<10^{3}$ of our computations. Consequently, we have an increase of type $\gamma$ from $36.0 \%$, with respect to the entire database, to $\frac{126}{153}=82.4 \%$, with respect to $T=4$.

The feature is even aggravated for conductors $f$ with $T=5$ prime factors, which exclusively occur in Table 36 . There are 4 similarity classes with $T=5$, namely [462], [546], [798], [858], with a total of 6 elements, all (100\%) with associated fields of type $\gamma$.

\section{Conclusions}

In this paper, it was our intention to realize Parry's suggestion ([1] p. 484) concerning a numerical investigation of pure quintic number fields $L=\mathbb{Q}(\sqrt[5]{D})$. For this purpose, we first developed theoretical foundations in a series of preparatory papers [6] [7] [8] [9] which expand the original germs in [12]. Since the non-Galois fields $L$ do not contain the full wealth of arithmetical structures, we had to consider their pure metacyclic normal closures $N=\mathbb{Q}\left(\zeta_{5}, \sqrt[5]{D}\right)$ of degree 20.

On the one hand, this enabled us to use the Galois cohomology of the unit group $U_{N}$ with respect to the relative automorphism group $G=\operatorname{Gal}(N / K) \simeq C_{5}$ over the cyclotomic field $K=\mathbb{Q}\left(\zeta_{5}\right)$ for defining 13 exhaustive and mutually exclusive differential principal factorization (DPF) types (Table 4 in $\S 3.1$ ), based on the unit norm index $\# H^{0}\left(G, U_{N}\right)=\left(U_{K}: N_{N / K}\left(U_{N}\right)\right)=5^{U}$, where $0 \leq U \leq 2$, which is connected with the order of the group of primitive ambiguous principal ideals $\# H^{1}\left(G, U_{N}\right)=\left(\mathcal{P}_{N / K}: \mathcal{P}_{K}\right)=5^{U+1}$ via the Takagi/Hasse/Iwasawa-Theorem on the Herbrand quotient of $U_{N}$, and on a natural decomposition $U+1=A+I+R$ into the dimensions of the $\mathbb{F}_{5}$-vector spaces of absolute, intermediate and relative differential principal factors ([6], eqn. (6.3)).

On the other hand, our theory of the relatively cyclic quintic Kummer extension $N / K$ as a 5-ring class field modulo the conductor $f=f_{N / K}$ over $K$ admitted the calculation of the multiplicity $m=m(f)$ of the conductor $f(\S 2)$, which is the number of non-isomorphic pure metacyclic fields $N$ sharing the 
common conductor $f$ and forming a multiplet $\left(N_{1}, \cdots, N_{m}\right)$ [8].

Equipped with this theoretical background, we were able to develop our Classification Algorithm 3.1 in $\S 3.2$, and to prove that it determines the DPF type of $L$ and $N$ in finitely many steps. It also decides whether the normal field $N$ is a Polya field or not. (It is known that $L$ is a (trivial!) Polya field if and only if it possesses class number $h_{L}=1$ [6].)

The algorithm was implemented as a Magma program script [3] [4] [5] and applied to the 900 fields $N$ with normalized fifth power free radicands in the range $2 \leq D<10^{3}$, after some preliminary experiments with Pari/GP [2]. The result is documented in the twenty Tables $6-25$ of $\S 3.4$. It is in perfect accordance with our theoretical predictions in 1991 [12]. Actually, each type occurs indeed, the most hardboiled type $\zeta_{2}$ not earlier than for the radicand $D=505$, and it is interesting to study the statistical distribution of the types in $\S 4.1$, Table 6. Concerning the reliability of our extensive database, and for understanding the degree of precision contained in our paper, we point out that all tables have been thoroughly double checked with respect to misprints and copy-paste errors for at least three times.

Nevertheless, after the completion of the statistics in $\S 4$, we came to the conviction that for deeper insight into the arithmetical structure of pure metacyclic fields $N$, the prime factorization of the class field theoretic conductor $f$ of the abelian extension $N / K$ (invariants $e_{0} ; t, u, v ; n, s_{2}, s_{4}$ ) and the primary invariants of all involved 5-class groups, partially given by the 5-valuations $\left(V_{L}, V_{M}, V_{N} ; E\right)$, should be taken in consideration. In the last section, we present a corresponding refinement of the DPF types in similarity classes ( $\S 4.2)$, which is very useful for various applications. It reduces the number 900 of investigated radicands to 134 representative prototypes ( $\S 4.5$ ). The remaining 766 radicands, which are bigger than the prototype of their similarity class, only reproduce the arithmetical invariants of the prototype and do not provide any additional relevant information. The rare phenomenon of non-elementary 5-class groups is documented in $\S 4.4$. Theorems on DPF-types of members of multiplets are proved in $\S 4.3$, and the striking dominance of type $\gamma$ with 3-dimensional (maximal) absolute principal factorization for radicands with three and more prime divisors is presented and discussed in $\S 4.6$.

\section{Acknowledgements}

We gratefully acknowledge that our research was supported by the Austrian Science Fund (FWF): project P 26008-N25. This work is dedicated to the memory of Charles J. Parry ( $† 25$ December 2010) who suggested a numerical investigation of pure quintic number fields. We are indebted to the anonymous referees for valuable suggestions concerning an improvement of the paper's layout.

\section{Conflicts of Interest}

The author declares no conflicts of interest regarding the publication of this paper. 


\section{References}

[1] Parry, C.J. (1975) Class Number Relations in Pure Quintic Fields. Symposia Mathematica, 15, 475-485.

[2] PARI Developer Group (2018) PARI/GP, Version 64 bit 2.11.1, Bordeaux. http://pari.math.u-bordeaux.fr

[3] Bosma, W., Cannon, J. and Playoust, C. (1997) The Magma Algebra System. I. The User Language. Journal of Symbolic Computation, 24, 235-265. https://doi.org/10.1006/jsco.1996.0125

[4] Bosma, W., Cannon, J.J., Fieker, C. and Steels, A., Eds. (2018) Handbook of Magma functions. Edition 2.24, Sydney.

[5] MAGMA Developer Group (2018) MAGMA Computational Algebra System. Version 2.24-3, Sydney. http://magma.maths.usyd.edu.au

[6] Mayer, D.C. (2018) Differential Principal Factors and Polya Property of Pure Metacyclic Fields. Accepted by Int. J. Number Theory. arXiv:1812.02436v1 [math.NT]

[7] Mayer, D.C. (2018) Similarity Classes and Prototypes of Pure Metacyclic Fields. Preprint.

[8] Mayer, D.C. (2018) Homogeneous Multiplets of Pure Metacyclic Fields. Preprint.

[9] Mayer, D.C. (2018) Coarse Cohomology Types of Pure Metacyclic Fields. arXiv:1812.09854v1 [math.NT]

[10] Barrucand, P. and Cohn, H. (1970) A Rational Genus, Class Number Divisibility, and Unit Theory for Pure Cubic Fields. Journal of Number Theory, 2, 7-21. https://doi.org/10.1016/0022-314X(70)90003-X

[11] Barrucand, P. and Cohn, H. (1971) Remarks on Principal Factors in a Relative Cubic Field. Journal of Number Theory, 3, 226-239. https://doi.org/10.1016/0022-314X(71)90040-0

[12] Mayer, D.C. (1991) Classification of Dihedral Fields. Preprint, Department of Computer Science, University of Manitoba, Manitoba.

[13] Mayer, D.C. (1993) Discriminants of Metacyclic Fields. Canadian Mathematical Bulletin, 36, 103-107. https://doi.org/10.4153/CMB-1993-015-x

[14] Kobayashi, H. (2016) Class Numbers of Pure Quintic Fields. Journal of Number Theory, 160, 463-477. https://doi.org/10.1016/j.jnt.2015.09.017

[15] Kobayashi, H. (2016) Class Numbers of Pure Quintic Fields. Ph.D. Thesis, Osaka University Knowledge Archive (OUKA).

[16] Aouissi, S., Mayer, D.C., Ismaili, M.C., Talbi, M. and Azizi, A. (2018) 3-Rank of Ambiguous Class Groups in Cubic Kummer Extensions. arXiv:1804.00767v4 [math.NT]

[17] Aouissi, S., Mayer, D.C. and Ismaili, M.C. (2018) Structure of Relative Genus Fields of Cubic Kummer Extensions. arXiv:1808.04678v1 [math.NT]

[18] Iimura, K. (1977) A Criterion for the Class Number of a Pure Quintic Field to Be Divisible by 5. Journal für die Reine und Angewandte Mathematik, 292, 201-210. 